\title{
Comprehensive two-dimensional liquid chromatographic analysis of anthocyanins
}

\author{
by \\ Chandré Monique Willemse
}

Dissertation presented for the degree of Doctor of Philosophy (PhD) in Chemistry in the Faculty of Science

at

Stellenbosch University

Supervisor: Prof. André. J. de Villiers

Co-supervisor: Dr. Maria. A. Stander

March 2015 


\section{Declaration}

By submitting this dissertation electronically, I declare that the entirety of the work contained therein is my own, original work, that I am the sole author thereof (save to the extent explicitly otherwise stated), that reproduction and publication thereof by Stellenbosch University will not infringe any third party rights and that I have not previously in its entirety or in part submitted it for obtaining any qualification.

Date: March 2015

Copyright @ 2015 Stellenbosch University

All rights reserved. 


\begin{abstract}
Anthocyanins are naturally occurring pigments responsible for the colour of many natural products, including grapes and wine. These pigments are important to the food industry and have been recognised for their nutritional value since they play an important role in the reduced risk of various chronic diseases in humans. Anthocyanins also play an important role in the aesthetic perception and quality of red wine. However, due to the large structural diversity of grape-derived anthocyanins and the many derivatives formed from these during wine ageing, the accurate analysis of wine pigments is extremely challenging. Reversed-phase liquid chromatography (RP-LC) is mostly used for anthocyanin analysis, although the technique often provides insufficient resolving power for complex mixtures of anthocyanins. In addition, the lack of commercially available standards and identical mass spectral characteristics hampers identification of these compounds. The coupling of multiple orthogonal separation systems in comprehensive 2-dimensional liquid chromatography (LC $\times \mathrm{LC}$ ) offers a more powerful approach for the separation of complex mixtures. The current work therefore focussed on exploring the potential of $\mathrm{LC} \times \mathrm{LC}$ for the improved analysis of anthocyanins and derived pigments in natural products and wine.
\end{abstract}

The first part of this work focussed on developing a hydrophilic interaction chromatography (HILIC) method as an alternative to RP-LC for the anthocyanin analysis. Following extensive optimisation, the method proved suitable for the analysis of a diverse range of anthocyanins in natural products. Significantly, it also showed alternative selectivity compared to RP-LC. The optimised HILIC method was then used in combination with RP-LC to develop an off-line LC $\times$ LC approach for anthocyanins. For this purpose, half-minute fractions of the HILIC effluent were collected and reinjected onto a RP-LC column. The off-line HILIC $\times$ RP-LC method demonstrated exceptionally high resolving power, as measured in terms of the practical peak capacity, with many compounds separated in two dimensions that co-eluted in 1-dimensional HPLC. Interestingly, group-type separation was also observed based on the degree and/or nature of glycosylation and acylation of anthocyanins. In the final part of the work, a systematic approach was used for the development and optimisation of and on-line HILIC $\times$ RP-LC method by using a 10-port switching valve to automatically transfer fractions between the two columns. This method was then coupled to high resolution mass spectrometry (MS) to allow the detailed investigation of anthocyanins and derived products in wine. Ninety four pigments were identified in one- and six-year old Pinotage wines based on HILIC $\times$ RPLC separation in combination with accurate mass MS data and fragmentation information. Significant differences in especially the content of derived pigments were observed between the wines. 
In summary, the methods developed in this work provide the means to improve anthocyanin analysis, and therefore also show promise for the detailed investigation of these important compounds and their alteration in natural products and their derived commodities. 


\section{Opsomming}

Antosianiene is natuurlike pigmente wat verantwoordelik is vir die kleur van baie natuurlike produkte, insluitende dié van druiwe en wyn. Hierdie pigmente is belangrik vir die voedsel industrie en word gereken vir hul voedingswaarde aangesien hulle ' $n$ belangrike rol speel in die verlaagde risiko van verskeie chroniese siektes onder die mens. Antosianiene speel ook 'n belangrike rol in die estetiese persepsie en kwaliteit van rooiwyn. Desnieteenstaande, as gevolg van die groot strukturele diversiteit van druifgeproduseerde antosianiene en die vele chemiese afgeleides wat uit hulle gevorm kan word tydens wyn bereiding en veroudering, is die akkurate analise van natuurlike wyn-pigmente uiters uitdagend. Omgekeerde-fase vloeistofchromatografie (RP-LC) word meestal gebruik vir die analise van antosianiene. Dié tegniek bied egter dikwels onvoldoende skeidingsvermoë vir komplekse mengsels van antosianiene en verwante molekules. Verder belemmer die onbeskikbaarheid van kommersiële standaarde en identiese massa spektrale eienskappe die identifikasie van hierdie verbindings. Die kombinasie van verskillende ortogonale skeidings meganismes in omvattende 2dimensionele vloeistofchromatografie $(\mathrm{LC} \times \mathrm{LC})$ bied egter 'n baie kragtiger benadering vir die skeiding van komplekse mengsels. Die huidige werk fokus dus op die ontginning van die potensiaal van LC $\times$ LC vir die verbeterde ontleding van antosianiene en verwante afgeleide pigmente in natuurlike produkte en wyn.

Die eerste deel van hierdie werk het gefokus op die ontwikkeling van 'n hidrofiliese interaksie chromatografiese (HILIC) metode as ' $n$ alternatief vir RP-LC analise van antosianiene. Na uitgebreide optimisering, is gevind dat die metode geskik is vir die ontleding van 'n verskeidenheid van antosianiene in natuurlike produkte. Van groot belang is dat dit ook alternatiewe selektiwiteit in vergelyking met RP-LC demonstreer. Hierdie geoptimiseerde HILIC metode word dan voorts gebruik in kombinasie met RP-LC vir die ontwikkeling van 'n af-lyn LC $\times$ LC benadering vir die analise van antosianiene. Hiervoor is half-minuut fraksies van die HILIC uitvloei opgevang en her-ingespuit op 'n RP-LC kolom. Dié af-lyn HILIC ×RP-LC metode toon buitengewoon hoë skeidingsvermoë, gemeet in terme van die bereikbare praktiese piek kapasiteit, met baie verbindings wat geskei is in die twee dimensies wat saam elueer in 1-dimensionele HPLC. Interessant genoeg is groep-tipe skeiding ook waargeneem gebaseer op die graad en / of aard van glukosilasie en asilering van die antosianiene. In die laaste deel van die werk, is ' $n$ sistematiese benadering gevolg vir die ontwikkeling en optimisering van 'n aan-lyn HILICXRP-LC deur gebruik te maak van 'n 10-poort oorskakelingsklep wat fraksies outomaties oordra tussen die twee kolomme. Die bogenoemde metode is ook verder gekoppel aan hoë resolusie massaspektrometrie (HR-MS) om 'n gedetailleerde ondersoek van antosianiene en hulle 
afgeleide verbindings in wyn moontlik te maak. Vier en negentig pigmente is in een- en ses jaar oue Pinotage wyne geïdentifiseer gebaseer op HILIC $\times$ RP-LC skeiding in kombinasie met akkurate massa MS data en fragmentasie inligting. Beduidende verskille in veral die inhoud van antosianien-afgeleide pigmente is tussen die wyne waargeneem.

Ter samevatting, die metodes ontwikkel in hierdie werk baan die weg om antosianien ontleding te verbeter en stel gevolglik die moontlikheid van selfs meer gedetailleerde studies van hierdie belangrike verbindings in natuurlike produkte in die vooruitsig. 


\section{Acknowledgements}

First and foremost, I would like to thank the Almighty, our Heavenly Father because it's only through the grace of GOD, his protection and guidance that I am where I am today.

I would also like to express my sincere gratitude to the following individuals for their invaluable contributions to this study:

I am eternally grateful to my supervisor Prof. André de Villiers for his guidance, advice and immense help offered throughout this study.

Thanks to my co-supervisor Dr Marietjie Stander, as well as Fletcher Hiten and Meryl Adonis for assistance with the LC-MS experiments and data analysis.

Mrs Heidi Duveskog, for guidance, support, advice and always being my sound board, I feel so indebted for the many lessons I've learned from you not just academically but on how I see the world, life and science.

A special thanks to SASOL and National Research Foundation (NRF) for the financial support.

Sincere thanks to the Separation Science Group (Stellenbosch University) for providing a friendly and constructive working environment.

Many thanks to the academic, technical and administrative staff of the Chemistry and Polymer Science department (Stellenbosch University) for all their assistance. A special thanks to Shafiek Mohamed, Deidre Davids, Roger Lawrence, Mary Johnson and Raymond Willemse for taking care of the administrative and laboratory matters.

I am thankful to Jochen Vestner for assisting with the processing of the $\mathrm{LC} \times \mathrm{LC}-\mathrm{MS}$ data.

I thank my parents for their words of wisdom, constant encouragement, support and love.

Last, but not least, my friends, family and anyone else whose name I might have omitted, thanks for your support you have all contributed to the successful completion of this work. 


\section{Table of Contents}

Declaration

Abstract

ii

Opsomming

iv

Acknowledgements

vi

Dissertation Layout

$\mathrm{X}$

\section{Chapter 1}

General Introduction and Objectives

1.1. Introduction

2

1.2. Aims and objectives

1.3. References

\section{Chapter 2}

Literature Review

2.1. Introduction

2.2. Phenolic Compounds

$\begin{array}{ll}\text { 2.2.1. Flavonoids } & 8\end{array}$

$\begin{array}{ll}\text { 2.2.2. Anthocyanins } & 8\end{array}$

$\begin{array}{ll}\text { 2.2.3. Anthocyanin solution chemistry } & 9\end{array}$

$\begin{array}{ll}\text { 2.2.4. Anthocyanins and derived pigments in grape and wine } & 10\end{array}$

2.2.4.1. Anthocyanin-proanthocyanidin derived products 11

2.2.4.2. Pyranoanthocyanins 
2.3. Analysis of anthocyanins

2.3.1. High performance liquid chromatography (HPLC)

2.3.2. Detection methods

2.4. Recent trends in anthocyanins and other phenolic compounds analysis

2.4.1. Ultra-high pressure liquid chromatography (UHPLC)

2.4.2. Alternative stationary phase morphologies

2.4.3. High temperature liquid chromatography (HTLC)

2.4.4. Multi-dimensional liquid chromatography (MDLC)

2.4.4.1. Peak capacity, sampling frequency and orthogonality

2.4.4.2. Method development and instrumental design in $\mathrm{LC} \times \mathrm{LC}$

2.4.4.3. Applications of $\mathrm{LC} \times \mathrm{LC}$ to phenolic analysis

\section{Chapter 3}

Results and Discussion

3.1. Introduction

3.2. Hydrophilic interaction liquid chromatographic analysis of anthocyanins

3.3 Comprehensive two-dimensional liquid chromatographic analysis of anthocyanins

3.4 Comprehensive two-dimensional HILIC $\times$ RP-LC-UV-MS analysis of anthocyanins and derived pigments in wine

\section{Chapter 4}


4.1. General conclusions

4.2. Recommendations for future studies

\section{Addenda}

Research Publications and Supporting Information

Addendum A: Hydrophilic interaction liquid chromatographic analysis of anthocyanins

C.M. Willemse, M.A. Stander, A. de Villiers, J. Chromatogr. A 1319 (2013) 127-140

Addendum B: Comprehensive two-dimensional liquid chromatographic analysis of anthocyanins

C.M. Willemse, M.A. Stander, A.G.J. Tredoux, A. de Villiers, J. Chromatogr. A 1359 (2014) 189-201

Addendum C: Comprehensive two-dimensional HILIC $\times$ RP-LC-UV-MS analysis of anthocyanins and derived pigments in wine

C.M. Willemse, M.A. Stander, J. Vestner, A.G.J. Tredoux, A. de Villiers, (submitted to Anal. Chem.) 


\section{Dissertation Layout}

The format of this dissertation is presented as a compilation of published work. Starting with a general introduction, which provides the reader with some insight on the subject matter, this is then followed by brief summaries of the content of each article and lastly some general conclusions and future recommendations. The structure of this dissertation is as follows:

Chapter 1 provides a short general introduction to the study at hand, highlighting the goals of the research.

Chapter 2 Provides background information on phenolic compounds, particularly anthocyanins and derived pigments, their different equilibrium forms, health effects and methods of analysis. Detailed discussions on multi-dimensional separations and aspects pertaining to this concept is addressed to help with the interpretation of results presented in Chapter 3.

Chapter 3 presents short summaries of the results (Addenda A-C) obtained in this study.

Chapter 4 presents general conclusions and recommendations for future studies.

Addenda A-C presents the publications resulting from this work and additional supporting information. 


\section{Chapter 1}

\section{General Introduction and Objectives}




\subsection{Introduction}

Anthocyanins are pigmented secondary plant metabolites found ubiquitously in nature. They are a sub-class of phenolic compounds and responsible for the red colour of grapes, wine and other many natural products. Anthocyanins are produced in grape skins during ripening [1], but during wine production and ageing the levels of grape-derived anthocyanins rapidly decrease as they react with other components, leading to the formation of more stable compounds that are responsible for the colour evolution of wine as well as loss of astringency $[2,3]$. Anthocyanins are natural antioxidants and have been shown to play a role in the prevention of cancer, heart disease and inflammatory diseases [4-6]. It is therefore important to develop improved methods for the accurate analysis and characterisation of these compounds.

Due to the structural diversity of anthocyanins and anthocyanin-derived pigments in natural products, however, their accurate qualitative analysis remains challenging. The most commonly used method for anthocyanin analysis is reversed-phase liquid chromatography (RP-LC), which is usually coupled to selective photodiode array (PDA) detection at 500-550 $\mathrm{nm}$. However, one of the major limitations of LC-PDA is that it does not provide sufficient structural information for the identification of individual anthocyanins. Nowadays RP-LC is increasing hyphenated to mass spectrometry (MS), which provides information on molecular mass and molecular structure, whereas tandem mass spectrometry (MS/MS) is useful for structural elucidation due to the selective fragmentation of anthocyanins [7,8]. Despite the power of RP-LC-MS, the technique is also associated with some limitations for the analysis of anthocyanins. Conventional RP-LC suffers from low optimal mobile phase flow rates and low chromatographic efficiency for anthocyanins under conventional conditions $[9,10]$. Furthermore, many anthocyanins have identical fragmentation patterns, complicating their identification by MS. For all of these reasons, more powerful separation methods for anthocyanin analysis are required.

Comprehensive two dimensional liquid chromatography ( $\mathrm{LC} \times \mathrm{LC})$ offers a powerful approach to improving resolution in liquid chromatography [11-17]. LC $\times \mathrm{LC}$ involves the transfer of fractions from a primary (first dimension) column to a secondary (second dimension) column for further separation. Improved resolution and selectivity is attained in the two-dimensional separation space by using two complementary, or orthogonal, separation methods. 
The aim of this study was to develop LC $\times \mathrm{LC}$ methods for the improved analysis of anthocyanins, focussing on wine anthocyanins and their derived pigments. To do so, the first step involved the development of an alternative separation method to RP-LC; hydrophilic interaction chromatography (HILIC) was used for this purpose, as the comprehensive combination of HILIC and RP-LC has been shown to provide a powerful method for phenolic analysis [11-13,18-20]. The applicability of the developed HILIC methodology was demonstrated for diverse anthocyanins found in a range of natural products. The next step then involved coupling of the HILIC method with RP-LC for the off-line LCXLC analysis of anthocyanins. Based on this work, the final step was the development and optimisation of an on-line HILIC $\times$ RP-LC method hyphenated to high resolution mass spectrometry (HR-MS) for the detailed analysis of anthocyanins and derived pigments in red wine.

\subsection{Aims and Objectives}

The overall aim of this work was to develop novel LC $\times$ LC methods for the improved determination of anthocyanins in various natural products, with particular emphasis on anthocyanin-derived products in red wine. For this to be achieved the following objectives had to be fulfilled:

i. The development of a HILIC method for anthocyanin analysis to be used in the first dimension of LCXLC. This includes evaluation of different stationary phases, mobile phase compositions and column temperatures to provide optimal chromatographic performance for anthocyanins.

ii. Off-line hyphenation of the optimised HILIC with RP-LC for the HILIC $\times$ RP-LC analysis of anthocyanins in natural products. This includes optimisation of column dimensions and flow rates in both dimensions as well as optimal hyphenation conditions to provide maximum resolution.

iii. The development of an on-line HILIC $\times$ RP-LC-UV-MS method for the detailed analysis of anthocyanin derived products in red wine. 


\subsection{References}

1. E. Garcia-Beneytez, E. Revilla, F. Cabello, Eur. Food Res. Technol. 215 (2002) 32.

2. T. C. Somers, Phytochem. 10 (1971) 2175.

3. E. Haslam, Phytochem. 19 (1980) 2577.

4. D. X. Hou, Curr. Mol. Med. 3 (2003) 149.

5. D. Bagchi, C.K. Sen, M. Bagchi, M. Atalay, Biochemistry (Mosc). 69 (2004) 75.

6. H. Wang, M.G. Nair, G.M. Strasburg, Y.C. Chang, A.M. Booren, J.I. Gray, D.L. DeWitt, J. Nat. Prod. 62 (1999) 294.

7. B. Abad-García, L. A. Berrueta, S. Garmón-Lobato, B. Gallo, F. Vicente, J. Chromatogr. A 1216 (2009) 5398.

8. M. M. Giusti, L. E. Rodriguez-Saona, D. Griffin, R.E. Wrolstad, J. Agric. Food Chem. 47 (1999) 4657.

9. A. de Villiers, D. Cabooter, F. Lynen, G. Desmet, P. Sandra, J. Chromatogr. A 1216 (2009) 3270.

10. A. de Villiers, D. Cabooter, F. Lynen, G. Desmet, P. Sandra, J. Chromatogr. A 1218 (2011) 4660 .

11. K.M. Kalili, J. Vestner, M.A. Stander. A. de Villiers, Anal. Chem. 85 (2013) 9107.

12. K.M. Kalili, A. de Villiers, J. Chromatogr. A 1289 (2013) 69.

13. P. Dugo, F. Cacciola, T. Kumm, G. Dugo, L. Mondello, J. Chromatogr. A 1184 (2008) 353.

14. P. Dugo, F. Cacciola, P. Donato, D. Airado-Rodríguez, M. Herrero, L. Mondello, J. Chromatogr. A 1216 (2009) 7483.

15. I. Francois, A. de Villiers, B. Tienpont, F. David, P. Sandra, J. Chromatogr. A 1178 (2008) 33.

16. R. A. Shellie, P.R. Haddad, Anal. Bioanal. Chem. 386 (2006) 405.

17. M. Kivilompolo, J. Pól, T. Hyötyläinen, LC-GC Europe. (2011) 232.

18. K.M. Kalili, A. de Villiers, J. Chromatogr. A 1216 (2009) 6274.

19. K.M. Kalili, A. de Villiers, J. Sep. Sci. 33 (2010) 853.

20. K.M. Kalili, A. de Villiers, J. Chromatogr. A 1289 (2013) 58. 


\section{Chapter 2}

\section{Literature Review}




\subsection{Introduction}

This chapter will introduce briefly the main topics of relevance to the work reported in the thesis. Topics covered include phenolic and anthocyanin chemistry, an overview of anthocyanins in wine and analytical methods for anthocyanin determination, with specific emphasis on liquid chromatography, multidimensional liquid chromatography and mass spectrometry. Note that the goal is not to present a comprehensive review of all relevant literature, but rather to provide a summary of the latest information and developments in each of these fields.

\subsection{Phenolic compounds}

Phenolic compounds are one of the most important groups of compounds occurring in plants, and are widely distributed in the plant kingdom. They are the most abundant secondary metabolites found in plants and comprise of more than 8000 different structures [1,2]. Phenolic compounds play an important role in determining the organoleptic properties of food, contributing for example to bitterness and astringency and colour. These compounds also exhibit anti-carcinogenic, anti-inflammatory and immune modulating activities, amongst others, and are important anti-oxidants [3].

Phenolics are characterised by the presence of an aromatic ring bearing one or more hydroxyl groups and can be divided into two classes, i.e flavonoids and non-flavonoids. Non-flavonoids are divided into simple phenolics, coumarins, xanthones, chalcones, stilbenes and lignans. Flavonoids are the most important group of phenolic compounds and are composed of seven sub-groups: flavones, flavonols, flavanones, isoflavones, flavanols, flavanonols and anthocyanins (Fig. 2.1). 


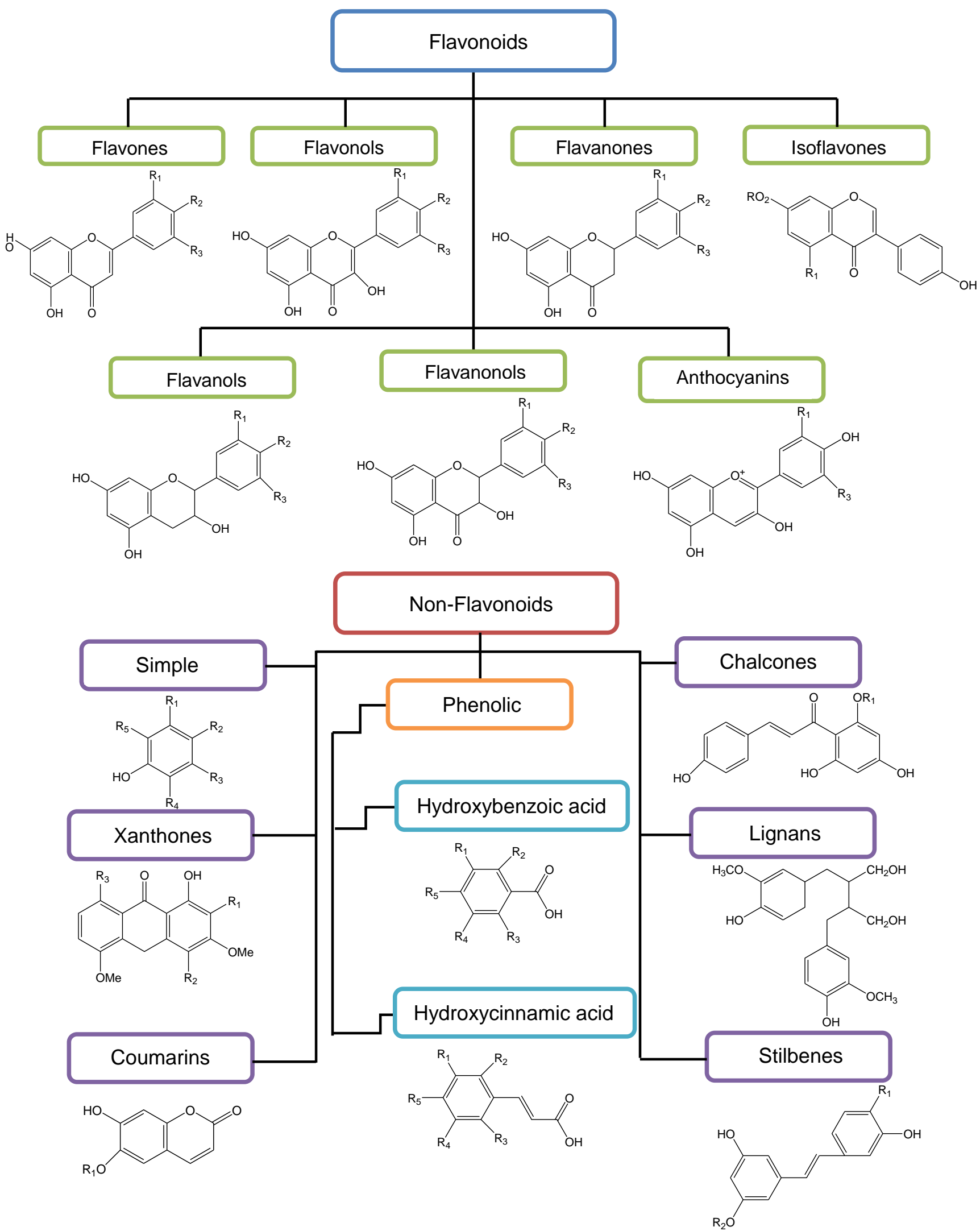

Figure 2.1: Classification of the different classes of phenolic compounds found in natural products[4]. 


\subsubsection{Flavonoids}

Flavonoids are not only one of the largest groups of phenolic compounds found in nature, but they might be the single most important group of phenolic compounds found in food. Numerous studies performed on flavonoids have shown that these compounds display biological activities such as antiallergenic, antiviral, anti-inflammatory and vasodilating effects. More than 9000 different flavonoids have been reported [5]. Flavonoids can be divided into different classes based on the degree of oxidation of their central pyran ring [5,6] (Fig. 2.1). The structure of a flavonoid contains the flavan nucleus consisting of 15 carbon atoms derived from a $\mathrm{C}_{6}-\mathrm{C}_{3}-\mathrm{C}_{6}$ skeleton. Anthocyanins are a unique class of flavonoids characterised by their flavylium cationic nature, which defines the properties of these water-soluble pigments.

\subsubsection{Anthocyanins}

The word anthocyanin is derived from the Greek words anthos (flower) and kyanos (blue). Anthocyanins are phenolic compounds responsible for the blue, violet and red colours of many flowers, fruits, leaves and stems. They are secondary metabolites, which occur in nature as water-soluble glycosides and acyl-glycosides of anthocyanidins. The basic anthocyanidin structure is a $\mathrm{C}_{6}-\mathrm{C}_{3}-\mathrm{C}_{6}$ skeleton consisting of a benzopyran ring with a phenolic ring at the $\mathrm{C}$ 2 position. Anthocyanins occur as positively charged flavylium (2-phenylbenzopyrylium) cations at acidic $\mathrm{pH}$, which is also what differentiates them from the rest of the flavonoid group. Anthocyanin research has intensified over the last two decades because of the importance of their potential health benefits, mainly their antioxidant properties [7,8]. Anthocyanins can be found in various fruits, vegetables, beverages and grains, and a diet rich in anthocyanins may reduce the risk of heart disease, cancer, visual acuity and age-related neuro-degenerated disorders [9-11].

The aglycone flavylium cation is called an anthocyanidin. When anthocyanidins are found in their glycosidic form they are known as anthocyanins. To date, roughly 30 different anthocyanidin structures have been identified [6], although approximately $90 \%$ of identified anthocyanins are based on the six most common anthocyanidin bases: pelaragonin, petunidin, 
cyanidin, peonidin, malvidin and delphinidin [6]. These anthocyanidins differ in the hydroxylation and methoxylation patterns on the B-ring, as shown in Fig. 2.2.<smiles>[R6]c1cc2c([R6])cc(O)cc2[o+]c1-c1cc([R6])c(O)c([R])c1</smiles>

$$
\begin{aligned}
& \text { Delphinidin (Dp): } \mathrm{R}_{1}=\mathrm{OH}, \mathrm{R}_{2}=\mathrm{OH}, \mathrm{R}_{3}=\mathrm{R}_{4}=\mathrm{H} \\
& \text { Cyanidin (Cy): } \mathrm{R}_{1}=\mathrm{OH}, \mathrm{R}_{2}=\mathrm{R}_{3}=\mathrm{R}_{4}=\mathrm{H} \\
& \text { Pelaragonidin (Pel): } \mathrm{R}_{1}=\mathrm{R}_{2}=\mathrm{R}_{3}=\mathrm{R}_{4}=\mathrm{H} \\
& \text { Petunidin (Pt): } \mathrm{R}_{1}=\mathrm{OCH}_{3}, \mathrm{R}_{2}=\mathrm{OH}, \mathrm{R}_{3}=\mathrm{R}_{4}=\mathrm{H} \\
& \text { Peonidin (Pe): } \mathrm{R}_{1}=\mathrm{OCH}_{3}, \mathrm{R}_{2}=\mathrm{R}_{3}=\mathrm{R}_{3}=\mathrm{H} \\
& \text { Malvidin (Mv): } \mathrm{R}_{1}=\mathrm{OCH}_{3}, \mathrm{R}_{2}=\mathrm{OCH}_{3}, \mathrm{R}_{3}=\mathrm{R}_{4}=\mathrm{H}
\end{aligned}
$$

Figure 2.2: Structures of the most common anthocyanidins occurring in nature.

The diversity of anthocyanin structures stem from differences in glycosylation, including the number, nature and positions of the glycoside units, as well as additional aliphatic and aromatic acylation of the attached sugars [6]. Anthocyanins are mostly mono-, di- or tri-glycosylated at the C-3, C-5, C-7, C-3', C-5' or more rarely the C-4' position [5,12-14]. The monosaccharides observed in glycosylated anthocyanins include glucose, rhamnose, xylose, galactose, arabinose and fructose [6]. With regard to acylation, the commonly observed aromatic acyl groups include $p$-coumaric, caffeic, ferulic, sinapic and 3,5-dihydroxycinnamic acids as well as $p$ hydroxybenzoic- and gallic acid. Acetic, malic, succinic, tartaric, oxalic and malic acid are the most frequent aliphatic acids involved in acylation [6]. Clearly, the enormous variety of anthocyanins found in nature makes them a very complex and diverse group of compounds.

\subsubsection{Anthocyanin solution chemistry}

Brouillard and co-workers $[15,16]$ have shown that anthocyanins occur in solution as several different species depending on the $\mathrm{pH}$ of the solution. The different chemical forms of anthocyanins are: flavylium cation $\left(\mathrm{AH}^{+}\right)$, quinoidal base (A), carbinol pseudobase (B) and chalcone $(\mathrm{C})$. The nature and the percentage of the predominant species depend on the $\mathrm{pH}$. At low pH (below 3) [17] the flavylium cation species, which absorb in the visible region of the spectrum at $\sim 520 \mathrm{~nm}$ and are therefore red, are the dominant species. With an increase in $\mathrm{pH}$, thermodynamic and kinetic competition between the hydration of the flavylium cation and proton transfer involving the acidic hydroxyl group occurs. The carbinol pseudo-base species 
is formed by hydration of the flavylium cation, which in turn undergoes further ring opening to yield the yellow chalcone (Fig. 2.3).<smiles>COc1cc(-c2oc3cc(=O)cc(O)c-3cc2OC(O)CCC(O)C(O)O)cc(OC)c1O</smiles>

$\underset{k_{12}}{\operatorname{proton} \operatorname{transfer}} \mid$<smiles>COc1cc(C2=[O+]C3C=C(O)C=C(O)C3C=C2OC2CC(O)C(O)C(O)CO2)cc(OC)c1O</smiles>

flavylium cation $\left(\mathrm{AH}^{+}\right)$

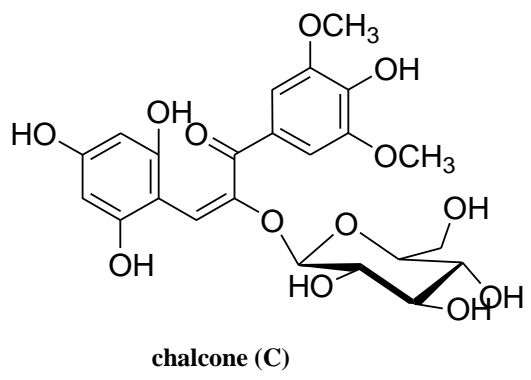<smiles>C=CCCC</smiles><smiles>COc1cc(C2(O)Oc3cc(O)cc(O)c3C=C2OC(O)COC2CC(O)C(O)CC2O)cc(OC)c1O</smiles>

carbinol pseudobase (B)

Figure 2.3: Equilibria involving the different chemical forms of anthocyanins in solution.

\subsubsection{Anthocyanins and derived pigments in grapes and wine}

Grape anthocyanins are responsible for the colour of red wine. The major anthocyanins present in grape skins are the glucosides of delphinidin $(\mathrm{Dp})$, cyanidin $(\mathrm{Cy})$, petunidin $(\mathrm{Pt})$, peonidin $(\mathrm{Pe})$ and malvidin (Mv), with malvidin-3-O-glucoside being the most abundant in Vitis vinifera species (Fig. 2.4). Direct condensation of anthocyanins yields oligomeric anthocyanins, several of which have been identified in the young red wine at low levels [18]. All of these compounds are extracted from the skins of grapes during wine maceration/formation. Malvidin contributes mostly to the colour of young red wine because of its high degree of methylation on the B-ring, making it the reddest anthocyanin [19]. Grape anthocyanins are not very stable in the wine matrix; during wine maturation and ageing the concentrations of these compounds decline with time due to degradation as well as through their reaction with a variety of other compounds 
present in wine [20]. This leads to the formation of more stable pigments that impart important changes to wine, notably in terms of taste and colour [21].

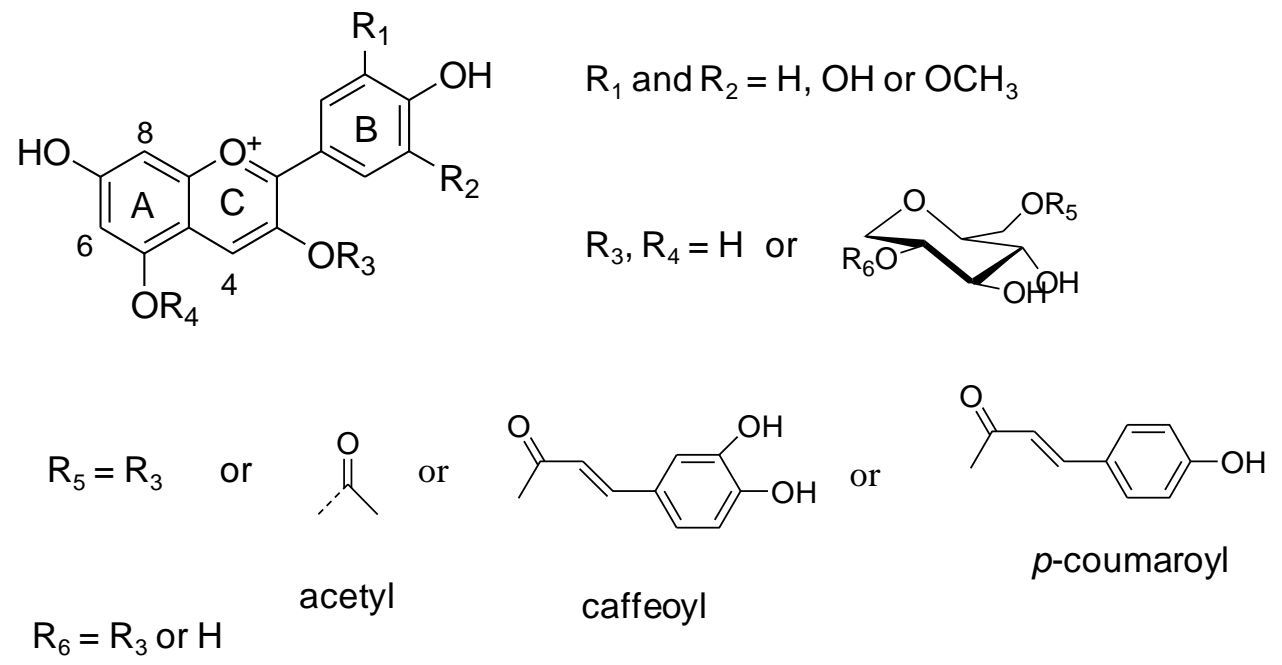

Figure 2.4: Structures of the common grape anthocyanins found in vitis vinifera species.

It has been shown that these transformations can occur via a range of pathways, including direct condensation between anthocyanins and flavanols [22], acetaldehyde-mediated condensation with flavanols [23-26] as well as through reactions with other low molecular weight compounds such as pyruvic acid [27-32], cinnamic acids [33-36], glycoxylic acid [37, 38], $\alpha-$ ketoglutaric acid [39] and acetone [36,39,40], to produce the so-called pyranoanthocyanins. The formation of these derived pigmented compounds is responsible for the change in colour of young red wines from purple-red to orange-red. These compounds are also more stable due to higher resistance to the effects of $\mathrm{pH}$ changes and bisulphite bleaching [28,30]. A brief discussion on each of these classes of derived pigments is presented in the following sections.

\subsubsection{Anthocyanin-proanthocyanidin derived products}

Phenolic compounds extracted from grape skins or seeds affect the colour of wine through the formation of coloured polymeric pigments with anthocyanins [41]. These pigments are formed via several pathways i.e., direct polymerisation of anthocyanins and flavan-3-ols or proanthocyanidins, or via reaction with acetaldehyde to form ethyl-bridged anthocyaninflavan-3-ols or proanthocyanidin adducts [41] or via the reaction with vinylflavanols [19,42]. During wine ageing the grape anthocyanins are steadily replaced by such oligomeric pigments. 
In addition, these polymeric pigments are important to the mouth-feel propertied of red wine [19].

The formation of direct proanthocyanidin-anthocyanin adducts occurs through coupling between the C-4 of an anthocyanin and either C- 6 or C-8 of a flavanol or procyanidin molecule (referred to as A-F condensation products), or between C-6 or C-8 of the anthocyanin and C-6 or C-8 of the flavanol/procyanidin, referred to as F-A condensation products $[43,44]$.

The acetaldehyde-mediated reaction between anthocyanins and flavonols yields reddish to violet polymeric pigments [45]. Acetaldehyde is the most abundant acetaldehyde found in red wine and originates from various sources such as yeast metabolism or the oxidation of ethanol. The anthocyanin moieties of these derived pigments are more protected against water attack and show higher stability with regard to bleaching by sulphur dioxide compared to monomeric anthocyanins [46].

Anthocyanin-vinylflavanols are formed from the reaction between vinylflavonols (originating typically from higher oligomers or the dehydration of flavonol-ethanol adducts) and anthocyanins. These compounds display a hypsochromic shift, with maximum absorbance at 490-510 $\mathrm{nm}$ and are therefore more orange than anthocyanins [19,47-49].

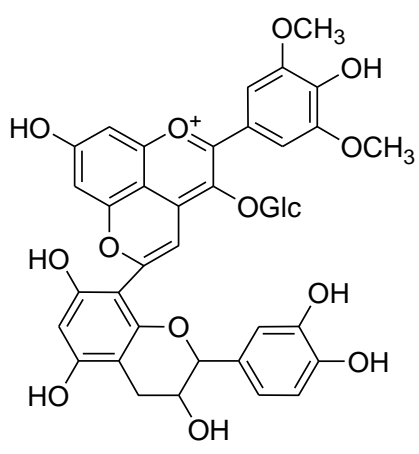

Anthocyanin vinylflavanol condensation product

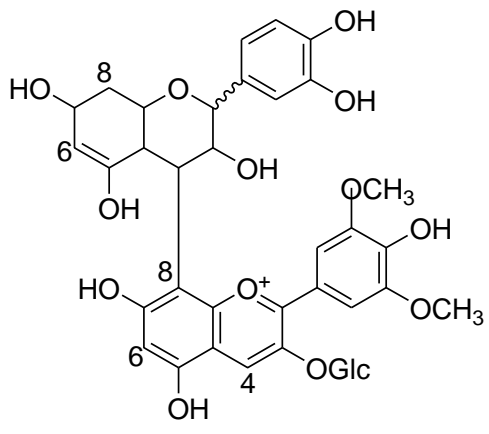

Directanthocyanin tannin adduct<smiles></smiles>

Acetaldehyde mediated tannins adduct

Figure 2.5: Structures of malvidin-glucoside-vinyl-(epi)catechin (vinylflavanol), malvidinglucoside-(epi)catchin (F-A direct anthocyanin tannin adduct) and malvidin-glucoside-ethyl(epi)catechin (acetaldehyde mediated tannin adduct). The flavanol units in these structures may also be procyanidin or prodelphinidin molecules for degree of polymerisation $\geq 2$. 


\subsubsection{Pyranoanthocyanins}

The direct reaction between grape anthocyanins and acetaldehyde, pyruvic acid and vinylphenols leads to the formation of pyranoanthocyanins [50-52], which typically are tawny coloured. In general pyranoanthocyanins are formed via the reaction of an anthocyanin molecule at position C-4 with a compound containing a polarizable double bond [53]. Pyranoanthocyanins therefore contain two heteroaromatic rings; the alteration at C-4 is responsible for the increased stability of these compounds. They show increased resistance to sulphur dioxide bleaching and oxidative degradation and therefore contribute significantly to the colour stability of wine $[50,52]$. Small amounts of pyranoanthocyanins have been found in red wines and grape pomace [27-29,42,54,55], but also in black carrot juice [56] and blood orange juice [57].

\section{(i) Vitisins}

The most prevalent group of pyranoanthocyanins found in red wine are usually vitisins [5860]. These compounds are formed during fermentation of must and later during maturation and ageing of red wine [61]. A-type vitisins are formed from the reaction between anthocyanins and pyruvic acid, whereas B-type vitisins are the result of the reaction with acetaldehyde [51] (Fig. 2.6). Oxovitisins are second-generation pigments formed through the nucleophilic attack of water on vitisin [62]. In addition, several oligomeric vitisin derivatives have been detected in aged ports $[47,63]$. 
<smiles>COC1=C2C=C(O)C=c3oc(-c4cc(C)c(O)c(C)c4)cc3=C1C=C(C(=O)O)O2</smiles>

Vitisin A<smiles>Cc1cc(C2=C(OCl)c3cc(=O)oc4cc(O)cc(c34)O2)cc(C)c1O</smiles>

Oxovitisin<smiles></smiles>

Vitisin B

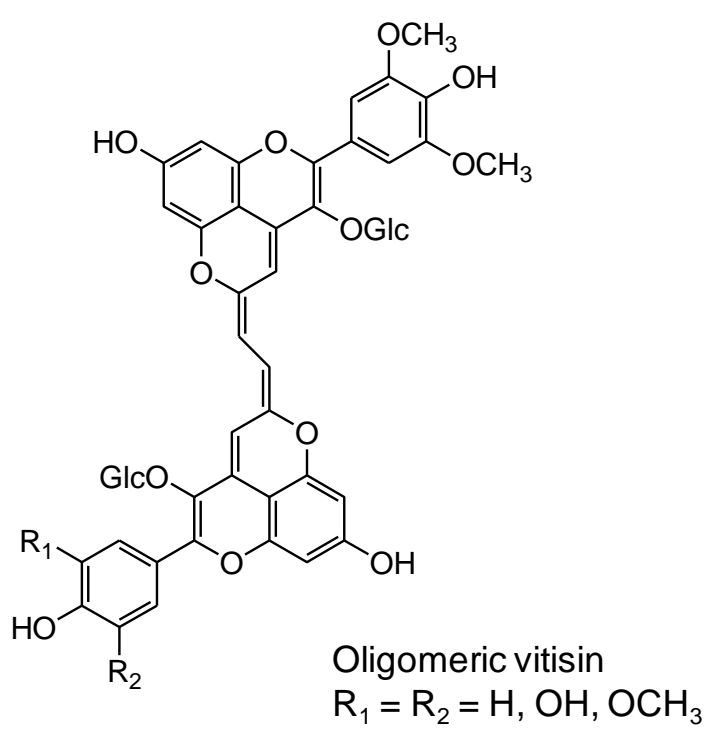

Figure 2.6: Structures of pyranoanthocyanins vitisin A, vitisin B, oxovitisins and oligomeric vitisins.

(ii) Anthocyanin-vinylphenol condensation products

The reaction between grape anthocyanins and several hydroxycinnamic acid such as coumaric acid, ferulic acid, caffeic acid and synaptic acid yields the formation of 4-vinylphenol, 4vinylguaiacol, 4-vinylcatechol, and 4-vinylsyringol adducts, respectively (Fig. 2.7) [64].

Malvidn-3-glucoside-4-vinylphenol was the first pyranoanthocyanin of this type identified in wine $[33,34]$. The formation of this compound pyranoanthocyanin occurs via the cyclo-addition reaction between the vinyl group of coumaric acid and carbon $\mathrm{C}-4$ and the $\mathrm{OH}$ at $\mathrm{C}-5$ of the anthocyanin. Other pathways include the direct reaction with vinylphenols produced from slow hydrolysis of the corresponding tartaric esters of hydroxycinnamic acids [65] or through enzymatic decarboxylation of hydroxycinnamic acids. In addition, several related structures differing only in the substitution pattern of the vinylphenol moiety (and therefore of the parent cinnamic acid) have been identified in wine $[35,66,67]$. Pinotin A (malvidin-glucoside- 
vinylcatechol), resulting from the reaction between malvidin-3-glucoside and caffeic acid, was the first compound of this class to be identified and was named after Pinotage wine in which it was identified. Higher concentrations of Pinotin A are found in aged compared to young wines [68].<smiles></smiles>

Malvidin-3-glucoside-vinylphenol<smiles></smiles>

Malvidin-3-glucoside-vinylcatechol<smiles></smiles>

Malvidin-3-glucoside-vinylguaicol<smiles></smiles>

Malvidin-3-glucoside-vinylsyringol

Figure: 2.7: Structures of several vinylphenol condensation products of malvidin-3-glucoside.

\subsection{Analysis of anthocyanins}

Various techniques such as thin layer chromatography (TLC) [69], capillary electrophoresis (CE) [70,71], have been used for the separation of anthocyanins, although high performance liquid chromatography (HPLC) is by far the most widely used chromatographic method for these compounds. Especially in the last 2 decades, mass spectrometry (MS) has also found extensive application in anthocyanin analysis, with ionisation methods such as matrix assisted laser desorption ionisation (MALDI) [72,73], electrospray ionisation (ESI) [74,75], atmospheric pressure chemical ionisation (APCI) [76,77] and continuous flow fast atom 
bombardment (CF-FAB) [78,79] having been applied. The main focus of the next section will therefore be on LC and LC-MS analysis of anthocyanins.

\subsubsection{HPLC analysis of anthocyanins}

The most widely used method for anthocyanin analysis, whether it be for analytical purposes or preparatory purposes, is HPLC [80]. With the use of HPLC, structurally similar species can be separated and identified in combination with various detectors such as UV-Vis, mass spectrometry (MS) and nuclear magnetic resonance (NMR) spectroscopy. Reversed phase liquid chromatography (RP-LC) is the mode of choice for anthocyanin analysis. RP-LC is usually performed on $\mathrm{C} 18$ columns, with binary mobile phase typically consisting of an acidified aqueous phase and either methanol or acetonitrile as organic modifier. For the RPLC separation of anthocyanins, highly acidic mobile phases are required, which helps improve peak shapes and resolution between closely eluting species [81]. For a typical RP-LC separation of anthocyanins, the separation is governed by hydrophobicity and stereochemistry. The general elution order is affected by three properties of the anthocyanin: (1) the anthocyanidin base, (2) number and nature of the attached glycosidic groups, and (3) the number and nature of acyl groups

The solution chemistry of anthocyanins has important implications for their chromatographic analysis. Because several may exist in the mobile phase, inter-conversion between these species will affect chromatographic performance [17]. Horváth et al. studied the effect of secondary equilibria on chromatographic peak shapes in detail $[82,83]$. Their results showed that, if the rate of inter-conversion is slow, the species will be resolved and multiple peaks will be detected. In contrast, rapid inter-conversion rates yield one peak, with the contribution of secondary equilibria to band broadening approaching zero. For intermediate rate constants, however, broad or distorted peaks are observed; for such reactions secondary equilibria have a negative impact on efficiency.

The Damköhler number $(\mathrm{Da})$ may be used to determine the contribution of secondary equilibria to the plate height. $D a$ is a dimensionless number defined by the residence time in the mobile phase and the relaxation time of the conversion reaction. For $D a$ numbers $<0.1$, the species will be completely separated and the reaction to no longer affects peak-broadening. Similarly, secondary equilibria do not significantly contribute to plate height for very fast conversion rates 
( $D a$ number $>100)$. Therefore the critical region define by $0.1<D a<100$ is where secondary equilibria will affect chromatographic performance (Fig. 2.8) [82].

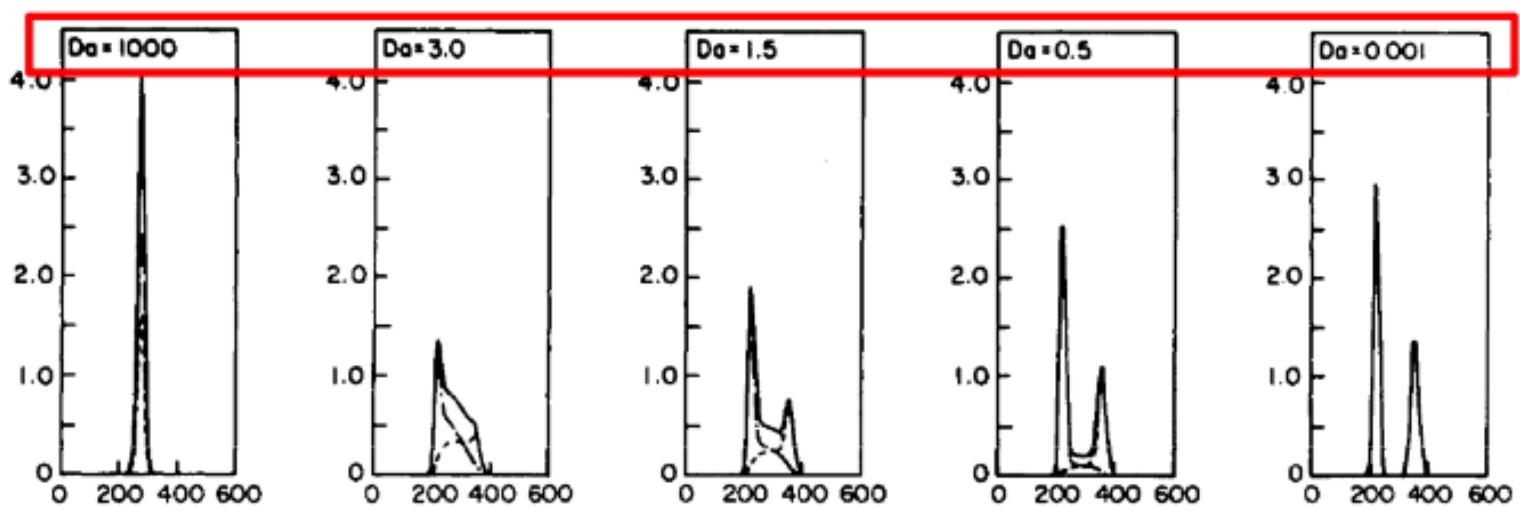

Fig. 2.8: Illustration of the effect of secondary equilibria on band broadening $[9,10]$.

de Villiers et al [17] studied the effect of anthocyanin secondary equilibria on their chromatographic separation in detail. Under highly acidic mobile phase conditions, only flavylium cation, carbinol pseudobase and quinoidal base species are present. These authors calculated $D a$ numbers for the proton transfer and hydration reactions, and showed that while the former does not contribute to band-broadening $\left(D a>2.1 \times 10^{7}\right)$, the latter conversion takes place on the same scale as the separation $(D a<100)$ and therefore leads to peak broadening. Two approaches to improving the chromatographic performance of anthocyanin analyses were reported by de Villiers et al $[17,42]$. In the first instance, using elevated temperature improves the chromatographic efficiency, since high temperatures result in an increase in the rate constant for the hydration reaction, thereby reducing band-broadening due to secondary equilibria [17,42]. In addition, columns packed with smaller particles provide faster mass transfer, which results in an improvement in chromatographic efficiency of anthocyanins, while at the same time increasing the linear velocity. However, relatively long columns need to be employed due to the dependence of the effect of secondary equilibria on the absolute retention time [42].

Hydrophilic interaction chromatography (HILIC) has in recent years increasingly been used as an alternative separation mode for polyphenolic analysis (although the technique has not been applied for anthocyanin analysis). HILIC is an aqueous variant of normal phase LC (NP-LC), which makes use of polar stationary phases (such as silica, bonded amide and diol phases) in 
combination with apolar mobile phases. Compounds are separated based on polarity [84]. The added benefits of HILIC include its suitability for polar compounds not sufficiently retained in RP-LC, excellent compatibility with UV and MS detection, and it also allows for hyphenation to other modes of HPLC in 2-dimensional LC. However, isomeric compounds differing for example in their glycosylation pattern are not separated in HILIC. For highly complex mixtures of phenolics, neither RP-LC nor HILIC provide sufficient resolving power to separate all compounds.

\subsubsection{Detection methods}

Detection is an important facet of any chromatographic analysis. An ideal detector should satisfy the following criteria: be selective, sensitive and be characterised by a linear response to solute concentration over a wide dynamic range. It has to be reliable with good stability and reproducibility, non-destructive, and have a small internal volume to reduce extra-column band broadening. The following discussion will focus on detectors used jointly with HPLC for the analysis of anthocyanins.

LC is most commonly coupled to UV-Vis detection; particularly photodiode array (PDA) detectors provide useful information on the nature of the anthocyanidin base as well as glycosylation and acylation patterns [85-87]. In addition, since anthocyanins absorb in the visible range around $500-550 \mathrm{~nm}$, they can selectively be detected at these wavelengths and can therefore be distinguished from other flavonoid classes [44,88]. One of the major shortcomings of LC-PDA is however that it does not provide sufficient structural information to allow identification of individual anthocyanins with certainty. Since the commercial availability of anthocyanin reference standards are also limited, mass spectrometry (MS) is increasingly being used as complementary detection method.

RP-LC hyphenated to MS is one of the most widely applicable analytical tools for anthocyanin determination, as it provides information on both the molecular mass and molecular structure, which aids in the identification. Various ionisation methods such as electrospray ionisation (ESI) [74,89-92], atmospheric pressure chemical ionisation (APCI) [77], continuous flow fast atom bombardment (CF-FAB) [79] and matrix assisted laser desorption ionisation (MALDI) [72] have been used. Quadrupole mass analysers are most widely applied, due to their robustness and low cost. However, high mass resolution analysers such as time-of-flight (TOF) 
instruments provide the analyst with exact mass and elemental formula information, thereby reducing uncertainty in compounds identification.

Since ESI is the most common form of ionisation used in the LC-MS analysis of anthocyanins, the following discussion will be limited to this mode of ionisation. ESI-MS detection of anthocyanins is virtually exclusively performed in positive ionisation mode. Under acidic mobile phase conditions anthocyanins are predominantly in the flavylium cationic form, which facilitates detection of their molecular cations $\left[\mathrm{M}^{+}\right]$in positive ionisation mode. For glycosylated anthocyanins, the aglycone anthocyanidin base is typically detected as base peak under collision induced dissociation (CID) conditions, following the neutural loss of $\mathrm{m} / \mathrm{z}, 162$ or 132 (glucoside or arabinoside), m/z 204 (acetyl-glucoside), $\mathrm{m} / \mathrm{z} 308$ (coumaroyl-glucoside), m/z 324 (di-glucoside or caffeoyl-glucoside) [44,93] (Fig. 2.9A). Fragmentation of acylated anthocyanins therefore involves cleavage of the intact acyl-glycosidic group [75]. Furthermore, under high collision energy conditions, characteristic fragmentation of the anthocyanidin base occurs which provides additional information on the substituents of the aglycone.

Fig. 2.9 illustrates the fragmentation patterns for anthocyanins under low and high energy CID conditions. In the notation used here, $\mathrm{Y}_{\mathrm{c}}^{\mathrm{a}}$, indicates the aglycone ion with the glycosidic bond specified by the subscript c for di- and tri-glucosides, and the position of glycosylation on the anthocyanidin base denoted by superscripts a and b[93]. Fig 2.9B illustrates the fragmentation involving the $\mathrm{C}$-ring for anthocyanidin aglycone cation under high collision energies. Rupture of the C-ring bond results in charged fragments containing A-rings (denoted $\left[{ }^{\mathrm{c}, \mathrm{d}} \mathrm{A}\right]^{+}$) and Brings, denoted as $\left[{ }^{\mathrm{c}, \mathrm{d}} \mathrm{B}\right]^{+}$. The position of the $\mathrm{C}$-ring fragmentation is specified by the superscript $\mathrm{c}$ and $\mathrm{d}$ in this notation (Fig. 2.9B). Malvidin, peonidin, petunidin, cyanidin and delphinidin all yield the $\left[{ }^{0.2} \mathrm{~A}_{0}\right]^{++}$radical cations at $m / z=150$ as a result if cleavage from $0 / 2$ C-ring bonds. Delphinidin is the only anthocyanidin that yields the $\left[{ }^{0.2} \mathrm{~A}_{0}\right]^{\cdot+}$ cation at $m / z=149$. Cyanidin and delphinidin both yield the $\left[{ }^{0.2} \mathrm{~B}_{0}\right]^{+}$ion at $m / z=137$ and 157 , respectively. The loss of $\mathrm{CO}$ from the $\left[{ }^{0.2} \mathrm{~B}_{0}\right]^{+}$ion to give rise to $\left[{ }^{0.2} \mathrm{~B}_{0}-\mathrm{CO}\right]^{+}$at $m / z=125$. The loss of B-ring yields a relatively intense ion at $m / z=179$ for malvidin and delphinidin. For cyanidin a weak fragment due to cleavage of the $\mathrm{C}-\mathrm{C}$ bond at position $0 / 3$ of the $\mathrm{C}$-ring results in the $\left[{ }^{0.3} \mathrm{~A}_{0}\right]^{+}$ion at $\mathrm{m} / \mathrm{z}=120$ $[44,93]$. 


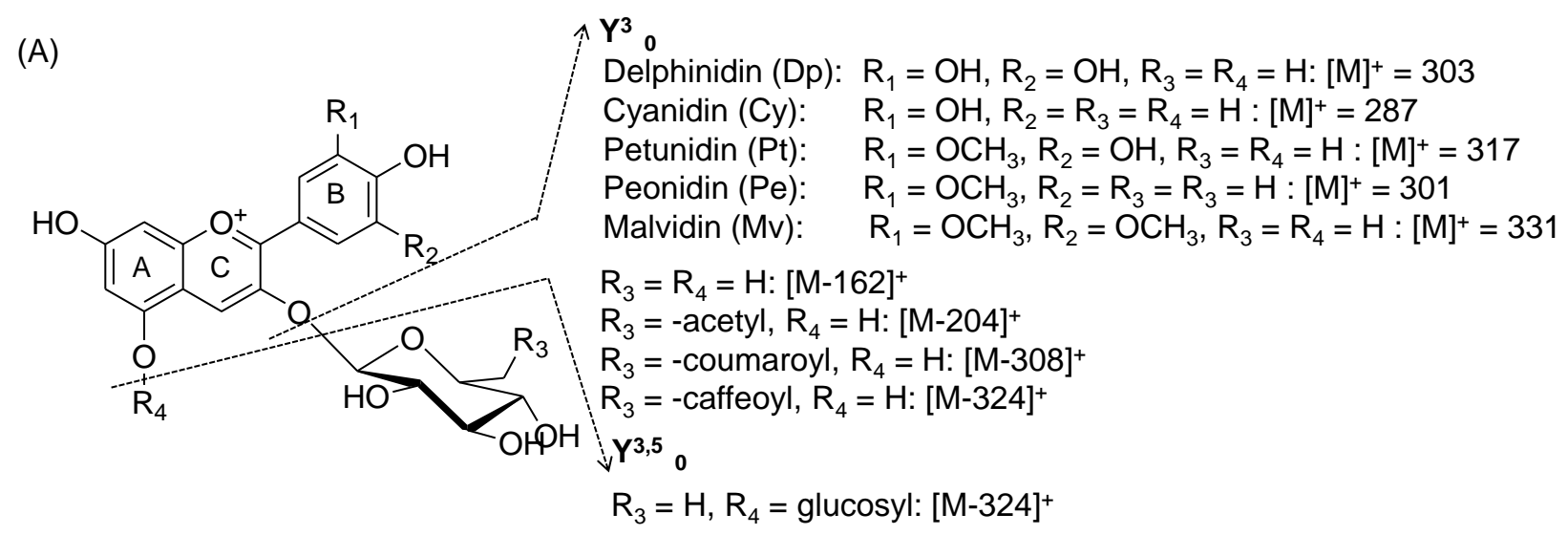

(B)

$[0,2 \mathrm{~A}]^{+*}$

Dp: $m / z 150$

Cy: $m / z 150$

Pt: $m / z 150$

Pe: $m / z 150$

Mv: $m / z 150$

[M-B-ring] ${ }^{+}$

Dp: $m / z 179$

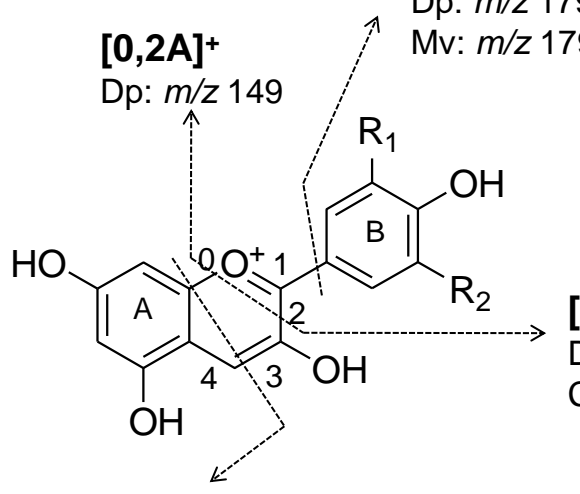

$\left.{ }^{0,2} \mathrm{~B}\right]^{+}$

Dp: $\mathrm{R}_{1}=\mathrm{R}_{2}=\mathrm{OH}: m / z 153$

Cy: $\mathbf{R}_{1}=\mathrm{OH}, \mathrm{R}_{2}=\mathrm{H}: m / z 137$

$\left[{ }^{0,3} \mathrm{~A}\right]^{+}$

[0,2B-CO]+

Cy: $m / z 120$

Dp: $\mathrm{R}_{1}=\mathrm{R}_{2}=\mathrm{OH}: m / z 125$

Figure 2.9: Illustrations of (A) neutral loss fragmentation of anthocyanins under low energy CID conditions and (B) fragmentation involving the C-ring for anthocyanidin aglycone cations under high collision energy [44,93]. Notation: Dp: delphinidin, Cy:cyanidin, Pt: petunidin, Pe: peonidin, and Mv: malvidin.

Tandem mass spectrometry (MS/MS) instruments such as triple quadrupole, ion trap and quadrupole-time-of-flight (Q-TOF) systems have been used extensively for the identification of phenolic compounds due to their high selectivity and structural elucidation properties [94]. These instruments allow the selection of an ion of a specific mass-to-charge $(\mathrm{m} / \mathrm{z})$ ratio to be selectively fragmented to ascertain structural features. LC-MS/MS analysis of anthocyanins has been thoroughly studied by $\mathrm{Wu}$ and Prior $[95,96]$, who used this technique to identify and 
characterise a large number of anthocyanins in common fruits, berries and grains. Controlled fragmentation of anthocyanins aids in compound identification by identifying the nature, number and order of the attached sugars, acyl groups and confirmation of the anthocyanidin base.

Studies involving anthocyanidin and anthocyanin derived pigment fragmentation, include work done by Alberts et al [44] and Pati et al [97], who made use of LC-MS/MS for the screening and identification of anthocyandin derived pigments in wine. Li et al [98] used LC-MS/MS for the identification of anthocyanins in grape juice and Cabernet Sauvignon grape pomace, whereas Huang et al [92] and De la Cruz et al [99] used LC-MS for the identification of anthocyanins in grapes. In fact, MS and MS/MS have been instrumental in identifying derived pigments in wine and model solutions, thereby allowing the elucidation of their formation pathways $[18,36,100-103]$.

\subsection{Recent trends in anthocyanin and other phenolic compound analysis}

As alluded to above, HPLC is the most widely used separation method for phenolic analysis. However, due to the limited chromatographic efficiency obtained on these instruments, complete chromatographic separation of complex samples, such as is the case for natural products, is not possible. During the last decade some significant developments, such as innovative stationary phase supports and instrumental improvements have helped to achieve higher throughput analyses and/or more efficient liquid chromatographic separations. A short discussion on some of the advances and their application on phenolic analysis will be addressed below.

\subsubsection{Ultra-high pressure liquid chromatography (UHPLC)}

The term UHPLC refers to LC separations performed at pressures above 400 bar. The popularity of the UHPLC stems from the fact that the technique can accommodate columns packed with sub-2 $\mu \mathrm{m}$ particles, which results in an increase in improved chromatographic performance. Columns containing smaller particles are packed more tightly, resulting in shorter diffusion distances and a more uniform flow through the column and therefore less peak broadening $[104,105]$. In terms of the van Deemter equation, which describes the origin of ban 
broadening in a packed column, the A (Eddy dispersion) and $\mathrm{C}$ (resistance to mass transfer) terms are reduced with a reduction in particle size. The effect of this can be seen in the plate height curves for 5, 3.5 and $1.7 \mu \mathrm{m}$ particles in the figure below.

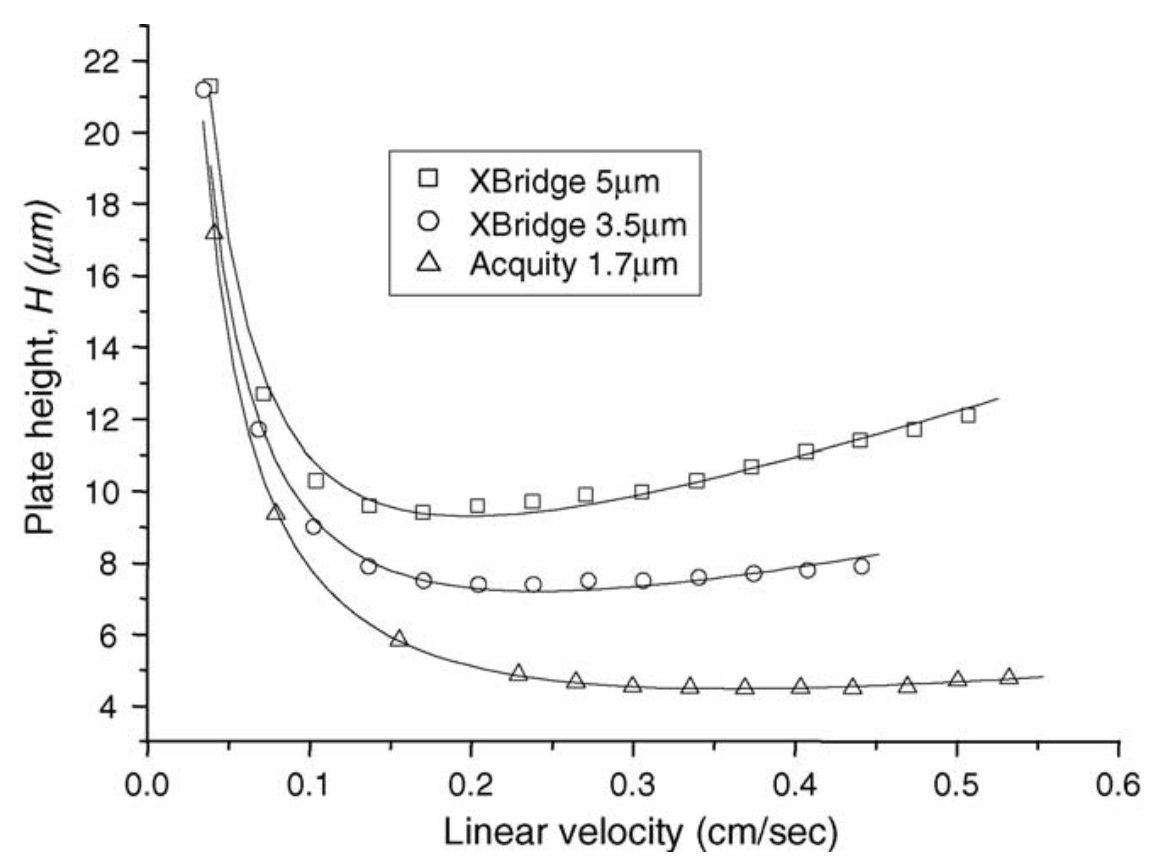

Fig. 2.10: Experimental van Deemter curves for 1.7, 3.5 and $5.0 \mu \mathrm{m}$ RP-LC columns illustrating the effect of a reduction in particle size [106].

Particle size reduction is associated with one important limiting factor: since the resistance to mobile phase flow through a column packed with sub- $2 \mu \mathrm{m}$ particles is higher, higher operating pressures are required. This is illustrated by Darcy's law, which relates the pressure drop across a column $(\Delta \mathrm{P})$ to the column permeability $\left(K_{0}\right)$, mobile phase viscosity $(\eta)$, column length $(L)$, mobile phase linear velocity $(u)$ and particle size $\left(d_{p}\right)$ :

$\Delta P=\frac{K_{o} \eta L}{d_{p}^{2}} u_{o}=\frac{K_{o} N H}{d_{p}^{2}} \mu_{o}$

Equation 2.1

According to equation 2.1 the pressure drop across a column is directly proportional to the column length and inversely proportional to the square root of $d_{p}$. Furthermore, since the optimal mobile phase linear velocity, $u_{o p t}$, is inversely proportional to $d_{p}$, the pressure required to operate at $u_{\mathrm{opt}}$ is proportional to $1 / d_{p}{ }^{3}$. Therefore, since an increase in column length coincides with an increase in pressure, the reduction in $d_{p}$ is often accompanied by a reduction 
in column length leading to faster analyses times. Under these conditions the gain in efficiency is not significant. However, when the length of the column is kept the same, higher efficiencies are obtained at the cost of increased instrumental pressure. The driving force behind the development of commercial UHPLC instrumentation and columns has therefore been the gain in speed and/or resolution attainable.

\subsubsection{Alternative stationary phase morphologies}

The most commonly used support for HPLC columns are fully porous silica particles. However, some limitations are associated with the use of these phases, such as frictional heating, which occurs at ultra-high pressures [107]. Therefore, attempts have been made to find alternative stationary phase morphologies to overcome some of the shortcomings of porous silica.

Monolithic columns are on such type of support, which consist of macroporous structures [108]. The benefit of these columns is that because of their through-pore structures, they have far less resistance to solvent flow, which enhances permeability of the column. Highly efficient chromatographic separations can be achieved through the use of long (meter-length) columns. In addition short monolithic columns can be operated at very high flow rates for an increase in analysis speed.

Superficially porous particles are made up of a non-porous solid core coated with a thin porous layer. Various particle sizes are nowadays available, ranging from 1.3-5 $\mu \mathrm{m}$. The major advantage of using a superficially porous phase is that their performance is similar to those of UHPLC columns packed with smaller particle sizes, but at lower operating pressures. The minimum plate height for these phases are lower than the $2 d_{p}$ limit common for fully porous phases, which is responsible for the fact that similar efficiencies can be obtained on larger particles compared to conventional phases. This means that these columns can also be used on conventional HPLC instruments. In addition, the recent availability of high-pressure resistant superficially porous phases extends their use to UHPLC instruments, where improved performance compared to UHPLC columns of the same particle size can be obtained [107]. 


\subsubsection{High temperature liquid chromatography (HTLC)}

Temperature provides an important means of improving chromatographic separation, also in the case of phenolic compounds. In LC the mobile phase viscosity is generally high, which causes slow diffusion and a strong resistance to mobile phase to flow and therefore high back pressure and high mass transfer resistance. All of these parameters can be reduced by increasing the analysis temperature. The effect of temperature on column performance is illustrated in Fig. 2.11, where plate height curves obtained on the same column at different temperatures are shown [109]. From this figure, it is evident that with an increase in temperature the maximum achievable efficiency, indicted by the minimum plate height value, remains virtually unchanged, while the optimal mobile phase linear velocity is shifted towards higher values, resulting in a reduction in analysis time. Even faster analyses may be achieved when using UHPLC in combination with HTLC.

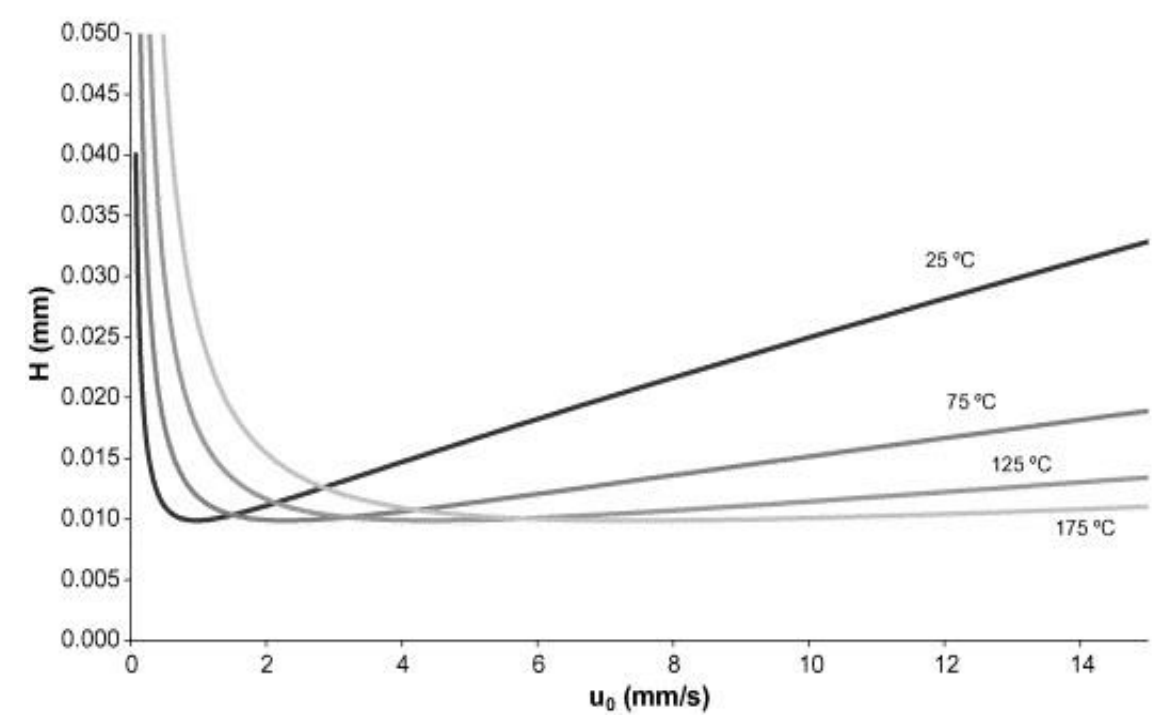

Fig. 2.11: Theoretical plate height curves illustrating the effect of temperature on the performance of a $5 \mu \mathrm{m}$ HPLC column [109].

The beneficial effects of elevated temperatures for the RP-LC separation of anthocyanins have been demonstrated by de Villiers et al [17,42]: an increase in optimal mobile phase linear velocity and a lower maximum plate height are observed at higher temperature. This is due to faster reaction kinetics for the hydration reaction at elevated temperatures, which results in a 
decreased contribution of secondary equilibria to band-broadening. Nevertheless, the analysis of anthocyanins at optimal mobile phase flow rates result in relatively long analysis times, although this might be partially offset by the use of UHPLC column. Alternatively, the low operating pressure implies that longer columns may be used as to benefit from efficiency gains $[17,42]$.

\subsubsection{Multi-dimensional liquid chromatography (MDLC)}

The term 'multidimensional' liquid chromatography (MDLC) refers to a family of techniques in which more than one separation mode are applied to separate the components in a sample. MDLC is characterised by an increase in resolving power compared to one-dimensional LC due to the combination of different separation mechanisms.

Two distinct modes of 2-D MDLC can be distinguished: heart-cutting 2-D LC and comprehensive 2-D LC (denoted LC $\times$ LC). In heart-cutting 2-D LC, only one or a few fractions of the target compounds are collected from the first dimension effluent and re-injected onto the second dimension column, typically by means of a loop interface. The remaining first dimension effluent is by-passed to waste. In $\mathrm{LC} \times \mathrm{LC}$, the entire first dimension effluent is collected and subjected to separation in the second dimension. This provides better separation of all compounds in the sample as opposed to only a selected fraction.

The benefits of $\mathrm{LC} \times \mathrm{LC}$ can be illustrated using the concept of peak capacity, which is defined as the maximum number of peaks that can fit into a given separation space between the first and last eluting peak [110]. Peak capacity is a theoretical metric, which is often used to measure the quality of a separation process. In heart-cutting 2-D-LC the peak capacity is the sum of the peak capacities in the first $\left({ }^{1} n_{c}\right)$ and second $\left({ }^{1} n_{c}+{ }^{2} n_{c}\right)$, whereas in LC $\times \mathrm{LC}$ it is the product of the peak capacities in the first and second dimension $\left({ }^{1} n_{c} \times{ }^{2} n_{c}\right)$.

LC $\times$ LC can be performed in either off-line, stop-flow or on-line modes. Off-line 2D LC is probably the easiest mode, which involves the collection of fractions of the first dimensional effluent (manually or with a fraction collector) and their reinjection onto the second dimension column. To perform an off-line 2-D LC separation only one HPLC system is required for all analyses. Compatibility of mobile phases is not a major concern, since immiscible solvents can be evaporated and redissolved in the second dimension injection solvent. Furthermore, off-line operation does not place restrictions on the chromatographic conditions such as column 
dimensions, flow rates and analysis times in the two dimensions. However, the major limitation of off-line $\mathrm{LC} \times \mathrm{LC}$ is that it is often tedious and time consuming, and there is always the possibility of sample contamination/loss and degradation. Automation of off-line LC $\times \mathrm{LC}$ is also hard to achieve.

Stop-flow LC $\times$ LC involves the direct transfer fractions from the primary column effluent onto the secondary column, after which the first dimension flow is stopped while the transferred fraction is analysed in the second dimension. When the separation in the second dimension is completed the first dimension flow is resumed again for the transfer of the following fraction.

On-line $\mathrm{LC} \times \mathrm{LC}$ is characterised by a much higher throughput, which results in more reproducible results. In this configuration fractions from the primary column are directly transferred to the second dimension column by employing a 2-position switching valve equipped with two loops. While a fraction is collected in one loop the content of the other is analysed on the second dimension column. Very fast second dimension analyses are therefore required for on-line $\mathrm{LC} \times \mathrm{LC}$, typically resulting in lower chromatographic performance compared to off-line LC $\times$ LC. Other limitations include the complexity of setting up an on-line 2-D system, especially the design of the interface. Not all separation modes are easily combined in on-line $\mathrm{LC} \times \mathrm{LC}$, due to mobile phase incompatibility.

Before addressing LC $\times$ LC method development and optimisation, a few key concepts relevant to this process will be discussed below.

\subsubsection{Peak capacity, sampling frequency and orthogonality}

As alluded to above, the major advantage of performing an $\mathrm{LC} \times \mathrm{LC}$ separation is the increased peak capacity, since for an ideal LC $\times$ LC separation system the peak capacity is multiplicative, i.e.,

$n_{c, 2 D}^{*}={ }^{1} n_{c} \times{ }^{2} n_{c}$

Equation 2.2

where $n_{\mathrm{c}, 2 \mathrm{D}}$, is the LC $\times \mathrm{LC}$ peak capacity and ${ }^{1} n_{\mathrm{c}}$ and ${ }^{2} n_{\mathrm{c}}$ the peak capacities of the first and second dimension separations, respectively. However, to attain this performance, several important criteria have to be met. The first of these involves the use of sufficient first dimension sampling frequencies. 
To maintain the first dimension resolution in a $2 \mathrm{D}$ configuration, a sufficient number of fractions across each peak eluting from the first dimension should be transferred. The sample or modulation period should therefore be shorter than the peak width emerging from the primary column. This is necessary since if any partially separated peaks in the first dimension are collected in the same fraction, their separation is completely lost. Therefore the general requirement is that the peaks eluting from the primary column should be sampled at least three to four times across their width to avoid loss of the first dimension resolution due to undersampling.

Murphy and co-workers [111] and Seeley [112] studied the effect of sampling rate on resolution in $\mathrm{LC} \times \mathrm{LC}$, with both groups coming to the same conclusion that when the first dimension peak is sampled a minimum of three times, the loss in resolution is minimal. In a later study by Horie and co-workers [113], it was shown that the best compromise between sampling rate and second dimension analysis time in on-line $\mathrm{LC} \times \mathrm{LC}$ can be achieved when each first dimension peak is sampled a minimum of two times. The reason for this is that, while increased sampling rates are required to maintain first dimension resolution, the use of shorter second dimension analyses comes with a reduction in peak capacity in this dimension, ultimately lowering the overall peak capacity. This facet is of the utmost importance in on-line $\mathrm{LC} \times \mathrm{LC}$ due to the fact that the second dimension separation should be completed within the sampling period. Thus for optimum system performance a compromise between sampling rate and second dimension analysis time is important. Since the two separations are performed independently in off-line and stop-flow LCXLC, the sampling rate can be selected independently of the second dimension analysis time. The only disadvantage is that higher sampling rates means more fractions are analysed in the second dimension, which results in longer overall analysis times.

Under-sampling of the first dimension peaks therefore contributes to loss of resolution and peak capacity in an LCxLC separation. To correct for under-sampling, the following relationship is used [114]:

$n_{c, 2 D}^{*}=\frac{{ }^{1} n_{c} \times{ }^{2} n_{c}}{\beta}$ Equation 2.3

where $\beta$ was used as the under-sampling correction factor, which can be calculated using the equation below [114]: 
$\beta=\sqrt{1+3.35\left(\frac{{ }^{2} t_{c} \times{ }^{1} n_{c}}{{ }^{1} t_{g}}\right)}$

Equation 2.4

where ${ }^{2} t_{c}$ is the second dimension cycle time and ${ }^{1} t_{g}$ is the gradient time in the first dimension [114]. When performing an off-line LC $\times$ LC separation the second dimension cycle time $\left({ }^{2} t_{c}\right)$ is replaced by the first dimension sampling time $\left({ }^{1} t_{s}\right)$.

A second important criterion to attain maximal performance in $\mathrm{LC} \times \mathrm{LC}$ is the requirement of sufficient orthogonality. Two separation methods are orthogonal if the separation mechanisms involved are independent of each other and show distinct retention profiles or provide different selectivities [115]. However, many separation modes have retention mechanisms in common, making the realisation of perfect orthogonality in practice difficult $[116,117]$. In essence, the advantage of an LCXLC separation is not fully utilised if the entire 2-dimensional separation space is not completely utilised, and this require the combination of orthogonal separations.

Several mathematical metrics have been developed to measure and take into account orthogonality in the estimation of LCxLC performance [118-122]. For this dissertation only the methods used herein will be discussed briefly, specifically the surface coverage (SC) methodology developed by Stoll and co-workers [120,121] and the convex hull method $[119,120]$. The Stoll method is based on dividing the separation space (defined as the 2dimensional space obtained using the range-scaled retention time in both dimension) into individual 'bins', and determining the SC value as the collective area encompassing all bins containing peaks. The number of bins $B$ should approximate the number of components $N$ in each sample [118]. Bins that do not contain compounds but are enclosed in the space covered by peaks are included. To obtain the $\mathrm{SC}_{\mathrm{S}}$, the number of bins within the enclosed area is then divided by the total number of bins.

The convex hull method [119] estimates $\mathrm{SC}_{\mathrm{CH}}$ by means of a rectangular hull, where the minimum convex hull is defined as the smallest polygon that can surround a group of peaks with none of the inner angles exceeding $180^{\circ}$ [120]. Both methods provide similar results and work for all kinds of data [118,120]. Fig. 2.12 illustrates graphically how surface coverage values are obtained using each of these methods. 

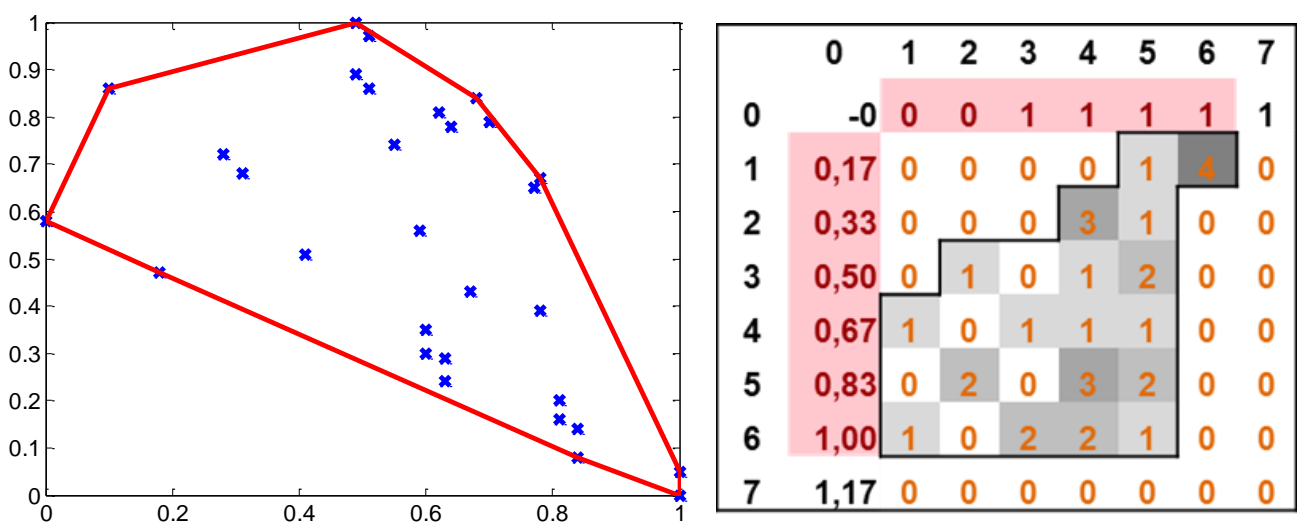

Fig. 2.12: Examples of surface coverage plots obtained using (a) the convex hull method and (b) the Stoll method and for the HILIC $\times$ RP-LC analysis of grape skin anthocyanins. The respective surface coverage values are $\mathrm{SC}_{\mathrm{CH}}: 0.482$ and $\mathrm{SC}_{\mathrm{S}}: 0.639$.

Both $\mathrm{SC}_{\mathrm{S}}$ and $\mathrm{SC}_{\mathrm{CH}}$ can be used as measures of the effective surface coverage, $f_{c}$, of the 2dimensional separation space. This value can then be used to obtain a more accurate measure of the performance of a $\mathrm{LC} \times \mathrm{LC}$ separation by correcting for finite orthogonality according to Equation 2.5:

$n_{c, 2 D}^{*}=\frac{{ }^{1} n_{c} \times{ }^{2} n_{c} \times f_{C}}{\beta}$

Equation 2.5

where the practical LC $\times$ LC peak capacity $\left(n_{c, 2 D}^{*}\right)$ takes into account first dimension undersampling as well as finite orthogonality.

Although both first dimension under-sampling and finite orthogonality should be taken into account when determining $\mathrm{LC} \times \mathrm{LC}$ performance, many literature reports omit these parameters or only account for one, which hampers accurate comparison between different methods.

\subsubsection{Method development and instrumental design in $L C \times L C$}

Prior to the coupling of two separations in $\mathrm{LC} \times \mathrm{LC}$, the individual 1-dimensional separations, should each be optimised according to the sample being used. In this process, all the parameters that influence peak capacity and practical aspects of the coupling of the two dimensions should also be taken into account. Parameters to consider include efficiency of the individual 
dimensions, orthogonality, sampling rate, column dimensions and flow rates and compatibility of the mobile phases.

In terms of the first dimension separation, the separation is defined by the maximum resolution required for a given application, since the analysis time is typically not limited in this dimension. To increase efficiency, the first dimension may consist of several columns coupled in series. Use of a narrow-bore HPLC column in the first dimension allows for minimal dilution and provides low flow rates that are compatible with second dimension injection volumes. The need for a pre-concentration step at the head of the second dimension column, or flow splitting, is therefore avoided. At the same time, complications associated with solvent incompatibility between different separation modes are minimised [117,123]. Wide-bore columns can be used in the first dimension, however, the effluent often should be split prior to the interface resulting in a decrease in sensitivity.

The general rule is to always use a second dimension column with a larger i.d. than the one used in the first dimension [124]. In the second dimension, wide-bore columns are therefore preferred to accommodate large fraction volumes and to enable the use of higher flow rates, which shortens re-equilibration times in the case of gradient analyses. In on-line LCXLC the analysis speed in the second dimension is of primordial importance. As alluded above, a sufficient number of fractions per first dimension peak width is required to maintain the peak capacity in this dimension. This implies that fast second dimension analysis should be performed, since the cycle time, defined as the time to perform a gradient and re-equilibrate the column, is equal to the sampling time. There are various ways to increase the analysis speed in the second dimension, such as using sub-2 $\mu \mathrm{m}$ phases and UHPLC instrumentation, monolithic columns, superficially porous phases and/or elevated temperatures $[125,126]$.

Once the individual separations have been optimised, the next step is to select the mode of coupling. Off-line LC $\times$ LC provides simplicity and the highest peak capacity, but at long analysis times. Stop-flow LC $\times$ LC is an automated alternative to off-line operation, whereas online LC $\times$ LC provides high throughput but generally lower performance than off-line or stopflow operation. The choice of hyphenation mode therefore depends on the time available and the required resolving power.

The major advantage of performing off-line 2-D LC is that longer analysis times can be used in the second dimension to achieve high peak capacities. This is as a result of both dimensions being carried out independently. For off-line method development the first dimension sampling 
time is chosen for an acceptable degree of under-sampling based on measured peak widths, whereas the second dimension cycle time is chosen to provide the required resolution in this dimension. Especially ${ }^{1} t_{s}$ (in other words, the number of fractions), but also ${ }^{2} t_{c}$ directly determines the total analysis time in on-line LC $\times$ LC [127]. Depending on the mobile phase properties and column dimensions and flow rates in both dimensions, the evaporation of solvent might be required prior to re-injection onto the second dimension column.

Method development for stop-flow LC $\times$ LC is essentially similar to off-line operation, with the added consideration that the overall peak capacity may be affected by band-broadening that occurs in the primary column during the stop-flow periods. The effective stop-flow periods experienced by each $1^{\text {st }}$ dimension peak as well as the effective diffusion coefficient of the target analyte(s) determines the extent of such band-broadening. This aspect may be quantified and used as an additional criterion in stop-flow development $[127,128]$

On-line LC $\times$ LC separation is usually performed with the help of switching valve (s) equipped with trapping loops. Fractions from the primary column are collected in one loop, while the content of the second loop is being analysed on the secondary column. This is a continuous process and occurs throughout the first dimension separation. In order to avert band displacement it is of the utmost importance that the second dimension separation be complete before transfer of the next fraction [129]. As a consequence on-line LC $\times \mathrm{LC}$ inherently involves a compromise between the degree of under-sampling (which requires short sampling times), and second dimension peak capacity (which requires long second dimension cycle times). This compromise is reflected in a plot of corrected 2-dimensional peak capacity $\left(n_{c, 2 D}^{*}\right)$ as a function of second dimension cycle time $\left({ }^{2} t_{c}\right)$ which is equal ( ${ }^{1} t_{s}$ in on-line LC $\times \mathrm{LC}$ ) as shown in Fig. 2.13. From this figure it is evident that $n_{c, 2 D}^{*}$ increases with increasing ${ }^{2} t_{c}$ up to a certain point - in this region increasing second dimension peak capacity with longer analysis times dominates the overall peak capacity. Beyond the maximum, excessive under-sampling leads to a reduction in 2-D peak capacity for longer ${ }^{2} t_{c}$ times. For a given on-line $\mathrm{LC} \times \mathrm{LC}$ system, there is therefore an optimal sampling time which will provide the highest practical peak capacity.

In terms of the mobile phase compatibility between two dimensions, if the injection volume onto the second dimension column is smaller than the fraction volume (i.e. the product of the first dimension flow rate and the sampling time), splitting before the second dimension column may be used in on-line and stop-flow LCXLC [127,130,131]. 


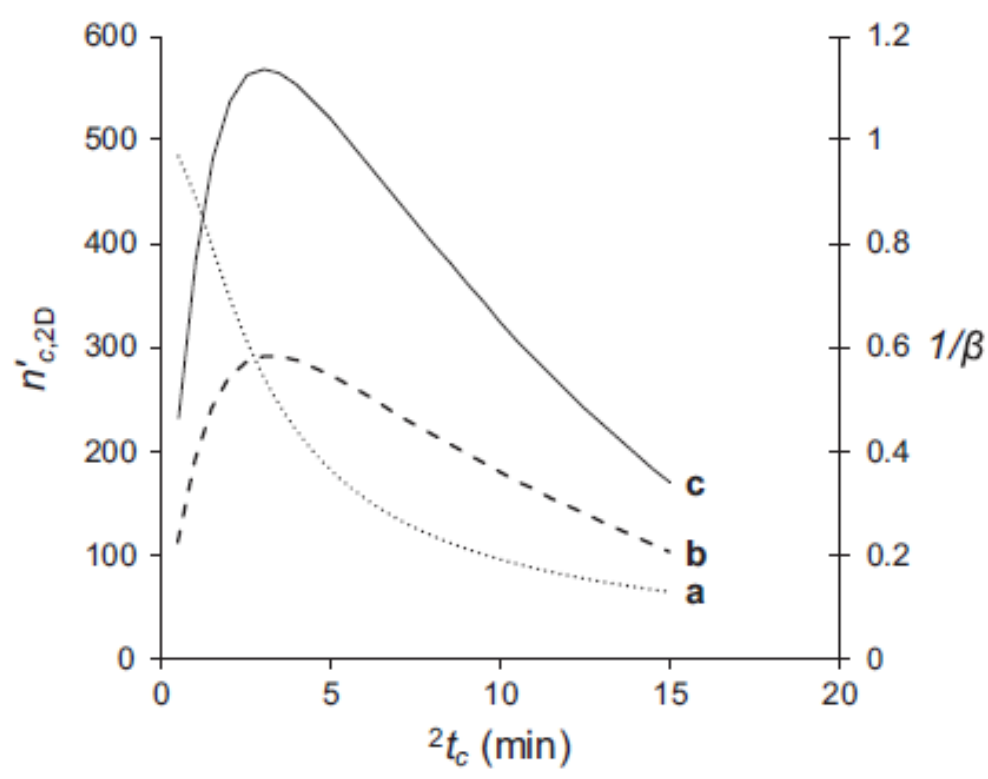

Figure 2.13: Plots of corrected LC $\times$ LC peak capacity (primary y-axis, lines b and $c$ at different first dimension flow rates) and the inverse of the under-sampling correction factor $(1 / \beta$, secondary y-axis, dotted line, curve a) as a function of second dimension cycle time $\left({ }^{2} t_{c}\right)$ for on-line HILIC $\times$ RP-LC analysis of procyanidins [127]. Further experimental conditions may be found in[127].

\subsubsection{Applications of $L C \times L C$ to phenolic analysis}

Due to the growing need for LC methods suitable for the analysis of complex samples, there has been an exponential increase in the use of $\mathrm{LC} \times \mathrm{LC}$ during the last decade. The technique has found application in various fields, including in the separation of complex biological samples [132], polymers[133-136], natural products [130,137,138], peptides [139,140], biomolecules [141], proteins [142], food [143], wine [144,145] etc. In this section, a brief overview of some application of $\mathrm{LC} \times \mathrm{LC}$ for phenolic analysis will be presented.

Various combinations of modes such as have been applied to the analysis phenolic compounds in a wide variety of samples, including NP-LC $\times$ RP-LC [146-148], HILIC $\times$ RP-LC $[130,137,138,149,150]$ and RP-LC $\times$ RP-LC $[144,151-156]$. The same separation mode may be applied in both the dimensions, as is the case with RP-LCXRP-LC, which has been demonstrated by Mondello and co-workers $[144,151,153,156]$. These authors performed on- 
line RP-LC $\times$ RP-LC of wine [144] and beer [145] phenolics. The advantage of this is that mobile phase incompatibility is minimised, although orthogonality is often poor.

For a more orthogonal combination NP-LC, HILIC or even size exclusion chromatography (SEC) can be used in combination with RP-LC, since these modes impart alternative separation selectivity due to their retention mechanisms. One of the major concerns when using NP-LC or HILIC is the solvent immiscibility (in the case of NP-LC) or mismatch between two dimensions. This factor is important, since the first dimension mobile phase is the injection solvent used in the second dimension when evaporation is not performed. Despite this limitation both NP-LC and HILIC have successfully been coupled to RP-LC and been applied for the LCXLC analyses of phenolic compounds. NP-LC $\times$ RP-LC was applied for the characterisation of psolarens and flavones in citrus oil [146,157] using MS detection. HILIC $\times$ RP-LC has been studied in detail by Kalili et al $[138,149,150]$ for the analysis of diverse phenolic compounds in natural products. These authors also hyphenated on-line HILIC $\times$ RP-LC with fluorescence and MS detection for the analysis of grape procyanidins [137] .

On-line LC $\times$ LC separation is most commonly used in phenolic analysis, with various separation modes being coupled [130,132,137,144,145,158,159] Different column stationary phases and mobile phases have been used in the on-line $\mathrm{LC} \times \mathrm{LC}$ analysis of a range of phenolic compounds in various samples such as wine [144], plant extracts, apples [160], beer [145], grape seeds [137] and cocoa [130].

Rather surprisingly, off-line LC $\times$ LC is less often used for phenolic analysis. Off-line HILIC $\times$ RP-LC was utilised by Kalili et al $[130,138,149,150]$, for the analysis of various phenolic compounds such as procyanidins, phenolic acids, flavanols etc., in a range of samples which included cocoa, apples, green tea and rooibos tea. Much higher peak capacities were obtained compared to the on-line configuration, however at longer analysis times.

Stop-flow LCXLC is the least often used mode of hyphenation, although it has been explored for phenolic analysis. Kalili et al $[127,130]$ investigated the potential of stop-flow LC $\times$ LC for the analysis of procyanidins in cocoa. These authors showed that additional first dimension band broadening due to stop-flow operation is not significant for these compounds, and reported similar peak capacity values compared to off-line LC $\times$ LC. Blahova et al [161] also explored stop-flow $\mathrm{LC} \times \mathrm{LC}$ for various phenolic standards and found good separation at slightly longer second dimension gradient times. 
The choice of detector used in LC $\times \mathrm{LC}$ depends on the analyte being detected and the separation mode used in the second dimension. UV is by far the most common detection method in the LC $\times$ LC analysis of phenolics [144,159,162], although the selectivity of fluorescence for procyanidins has been exploited in combination with $\operatorname{LC} \times \operatorname{LC}[130,137,138,149,150]$, and several studies have used MS in combination with LCxLC [137,138,163]. Hyphenation of $\mathrm{LC} \times \mathrm{LC}$ with MS is expected to find increasing application in phenolic analysis.

From the above short summary, it is evident that improved chromatographic separation is obtained by $\mathrm{LC} \times \mathrm{LC}$ for phenolic analysis. Clearly $\mathrm{LC} \times \mathrm{LC}$ will continue to play an important role in the analysis of complex phenolic fractions in the future. Although $\mathrm{LC} \times \mathrm{LC}$ has not to date been employed for anthocyanins, it would seem from the reports cited above that HILIC $\times$ RP-LC would be a promising approach for exploiting the benefits of $\mathrm{LC} \times \mathrm{LC}$ in anthocyanin analysis. 


\subsection{References}

1. W. Hümmer, P. Schreier, Mol. Nutr. Food Res. 52 (2008) 1381.

2. C.D. Stalikas, J. Sep. Sci. 30 (2007) 3268.

3. S.A. Lazarus, G.E. Adamson, J.F. Hammerstone, H.H. Schmitz, J. Agric. Food Chem. 47 (1999) 3693.

4. K.M. Kalili, A. de Villiers, J. Sep. Sci. 34 ( 2011) 854.

5. C.A. Williams, R.J. Grayer, Nat. Prod. Rep. 21 (2004) 539.

6. Ø. M. Andersen, M. Jordheim., The anthocyanins, in: Ø.M. Andersen, K.R. Markham (Eds.), Flavonoids: Chemistry, Biochemistry and Applications, CRC Press Boca Raton (2006), p.471. 7. J.A. Vinson, L. Zubik, P. Bose, N. Samman, J. Proch, J. Am. Coll. Nutr. 24 (2005) 44.

8. R.A. Moyer, K.E. Hummer, C.E. Finn, B. Frei, R.E. Wrolstad, J. Agric. Food Chem. 50 (2002) 519.

9. W. Yi, C.C. Akoh, J. Fischer, G. Krewer, J. Agric. Food Chem. 54 (2006) 5651.

10. M.E. Olsson, K-E. Gustavsson, S. Anderson, A. Nilsson, R-D. Duan, J. Agric. Food Chem. 52 (2004) 7264.

11. H.A. Hassan, A.F. Abdel-Aziz, Food Chem. Toxicol. 48 (2010) 1999.

12. R. Brouillard, Flavonoids and flower colour, in: J.B. Harborne (Ed), The Flavonoids: Advances in Research since1980, Chapman \& Hall, London (1988) p 525.

13. T. Fossen, R. Slimestad, Ø. M. Andersen, Phytochem. 64 (2003) 1367.

14. Ø. Bjorøy, T. Fossen, Ø. M. Andersen, Phytochem. 68 (2007) 640.

15. R. Brouillard, B. Delaporte, J. Am. Chem. Soc. 99 (1977) 8461.

16. R. Brouillard, J.-E. Dubois, J. Am. Chem. Soc. 99 (1977) 1359.

17. A. de Villiers, D. Cabooter, F. Lynen, G. Desmet, P. Sandra, J. Chromatogr. A 1216 (2009) 3270 .

18. C. Alcalde-Eon, M.T. Escribano-Bailon, C. Santos-Buelga, J.C. Rivas-Gonzalo, J. Mass Spectrom. 42 (2007) 735.

19. F. He, N.N. Liang, L. Mu, Q.H. Pan, J. Wang, M.J. Reeves, C.Q. Duan, Molecules. 17 (2012) 1483.

20. T. C. Somers, Phytochem. (1971) 2175.

21. E. García-Puente Rivas, C. Alcalde-Eon, C. Santos-Buelga, J.C. Rivas-Gonzalo, and M.T. Escribano-Bailón, Anal. Chim. Acta. 563 (2006) 215.

22. T. C. Somers, Phytochem. 10 (1971) 2175.

23. H. Liao, Haslam, E, J. Sci. Food Agric. 59 (1992) 299. 
24. C.F. Timberlake, P. Bridle, Am. J. Enol. Vitic. 27 (1976) 97.

25. J. Bakker, A. Picinelli, P. Bridle, Vitis. 32 (1993) 111.

26. J.C. Rivas-Gonzalo, S. Bravo-Haro, C. Santos-Buelga, J. Agric. Food Chem. 43 (1995) 1444.

27. J. Bakker, P. Bridle, T. Honda, H. Kuwano, N. Saito, N Terahara, C.F. Timberlake, Phytochem. 44 (1997) 1375.

28. J. Bakker, C. F. Timberlake, J. Agric. Food Chem. 45 (1997) 35.

29. H. Fulcrand, C. Benabdeljalil, J. Rigaud, V. Cheynier, M. Moutounet, Phytochem. 47 (1998) 1401.

30. C. Romero, J. Bakker, J. Agric. Food Chem. 47 (1999) 3130.

31. N. Mateus, A.M.S. Silva, J. Vercauteren, V. de Freitas, J. Agric. Food Chem. 49 (2001) 4836.

32. N. Mateus, V. de Freitas J. Agric. Food Chem. 49 (2001) 5217.

33. P.J. Cameira-dos-Santos, J.M. Brillouet, V. Cheynier, M. Moutounet, J. Sci. Food Agric. 70 (1996) 204.

34. H. Fulcrand, P.J. Cameira-dos-Santos, P. Sanri-Manchado, V. Cheynier, J. Favre-Bonvin, J. Chem. Soc. Perkin Trans. 1 (1996) 735.

35. M. Schwarz, G. Jerz, P. Winterhalter, Vitis. 42 (2003) 105.

36. Y. Hayasaka, R.E. Asenstorfer, J. Agric. Food Chem. 50 (2002) 756.

37. H. Fulcrand, V. Cheynier, J. Oszmianski, M. Moutounet, Phytochem. 46 (1997) 223.

38. N.E. Es-Safi, C.L. Guernevé, B. Labarbe, H. Fulcrand, V. Cheynier, M. Moutounet, Tetrahedron Lett. 40 (1997) 5869.

39. C. Benabdeljalyl, V. Cheynier, H. Fulcrand, A. Hakiki, M. Mosaddak, M. Moutounet, Sci. Aliments. 20 (2000) 203.

40. Y. Lu, L.Y. Foo, Tetrahedron Lett. 42 (2001) 1371.

41. R.S. Jackson, Wine Science: Principles and Applications, Elsevier-Academic Press.Oxford, UK p.287.

42. A. de Villiers, D. Cabooter, F. Lynen, G. Desmet, P. Sandra, J. Chromatogr. A 1218 (2011) 4660 .

43. J.S. Jensen, S. Demiray, M. Egebo, A.S. Meyer, J. Agric. Food Chem. 56 (2008) 1105.

44. P. Alberts, M.A. Stander, A. de Villiers, J. Chromatogr. A 1235 (2012) 92.

45. N.M.C. Sousa, A.M.S. Silva, A.M. Gonzalez-Paramas, C. Buelga, V. de Freitas, Food Chem. 102 (2007) 1344. 
46. T. Escribano-Bailon, M. Aivarez-Garcia, J.C. Rivas-Gonzalo, F.J. Heredia, C. SantosBuelga, J. Agric. Food Chem. 49 (2001) 1213.

47. A. Marques, M.P. Serratosa, J. Merida, J. Chem. 2013 (2012) 1.

48. L. Cruz, N. Teixeira, A.M. Silva, N. Mateus, J. Borges, V. De Freitas, J. Agric. Food Chem. 56 (2008) 10980.

49. L. Cruz, A.M.S. Silva, N. Mateus, V. de Freitas, Lett. Org. Chem. 5 (2008) 530.

50. V. de Freitas, N. Mateus, Anal. Bioanal. Chem. 401 (2011) 1463.

51. H. Fulcrand, C. Benabdeljalil, J. Rigaud, V. Cheynier, M. Moutounet, Phytochem. 47 (1998) 1401.

52. M. Rentzsch, M. Schwartz, P. Winterhalter, Trends Food Sci. Technol. 18 (2007) 526.

53. D. Von Baer, M. Rentzsch, M.A. Hitschfeld, C. Mardones, C. Vergara, P. Winterhalter, Anal. Chim. Acta. 621 (2008) 52.

54. N. Mateus, J. Oliveira, M. Haettich-Motta, V. de Freitas, J. Biomed. Biotechnol. 5 (2004) 55. V. Cheynier, Flavonoids in Wine, Ø. M, Andersen, K.R. Markham (Eds), In Flavonoids: Chemistry, Biochemistry and Applications, CRC Press, Boca Raton, 2006, p263.

56. M. Schwarz, V. Wray, P. Winterhalter, J. Agric. Food Chem. 52 (2004) 5095.

57. S. Hillebrand, M. Schwarz, P. Winterhalter, J. Agric. Food Chem. 52 (2004) 7331.

58. C.F. Timberlake, J. Bakker, J. Agric. Food Chem. 45 (1997) 35.

59. C. Romero, J. Bakker, J. Agric. Food Chem. 47 (1999) 3130.

60. J. Bakker, P. Briddle, T. Honda, H. Kuwano, N. Saito, N. Terahara, C.F. Timberlake, Phytochem. 44 (1997) 1375.

61. V. de Freitas, N. Mateus, Anal Bioanal Chem. 401 (2011) 1463.

62. J. He, J. Oliveira, A.M Silva, N. Mateus, V. De Freitas, J. Agric. Food Chem. 58 (2010) 8814.

63. J. Oliveira, J. Azevedo, A.M.S. Silva, N. Teixeira, L. Cruz, N. Mateus, V, de Freitas, J. Agric. Food Chem. 58 (2010) 5154.

64. J. Bakker. A. Picinelli, P. Bridle, Vitis. 33 (1994) 31.

65. T. Somers, E. Verette, K. Pocock, J. Sci. Food Agric. 40 (1987) 67.

66. M. Rentzch, M. Schwartz, P. Winterhalter, I. Hermosin-Gutierrez., J. Agric. Food Chem. 55 (2007) 4883.

67. C. Alcalde-Eon, M. T. Escribano-Bailon, C. Santos-Buelga, J. C. Rivas-Gonzalo, Anal. Chim. Acta. 513 (2004) 305.

68. M. Schwarz, T.C. Wabnitz, P. Winterhalter, J. Agric. Food Chem. 51 (2003) 3682. 
69. G. Mazza, E. Minitiati., Introduction. In anthocyanins in fruits, vegetables, and grains, Ed, Boca Raton, CRC Press. FL, 1993.

70. F.J.V. Gomez, R.P. Monasterio, V.C.S. Vargas, M.F. Silva., Electrophoresis. 33 (2012) 2240.

71. P. Bednar, B. Papouskova, L. Muller, P. Bartak, J. Stavek, P. Pavlousek, K. Lemr, J. Sep. Sci. 28 (2005) 291.

72. V. Ivanova, A. Dornyei, M. Stefova, T. Stafilov, B. Vojnoski, F. Kilar, L. Mark, Food Anal. Methods. 4 (2011) 108.

73. H. Wang, M.G. Nair, G.M., Strasburg, Y.C. Chang, A.M. Booren, J.I. Gray, D.L. DeWitt, J. Nat. Prod. 62 (1999) 294.

74. V. Cheynier, T. Doco, H. Fulcrand, S. Guyot, E. le Roux, J. M. Souquet, J. Rigaud, M. Moutounet, Analysis. 25 (1997) M32.

75. M.M. Giusti, L. E. Rodrıguez-Saona, D. Griffin, R. E. Wrolstad, J. Agric. Food Chem. 47 (1999) 4657.

76. W. Mullen, M.E. Lean, A. Crozier, J. Chromatogr. A 966 (2002) 63.

77. A. Baldi, A. Romani, N. Mulinacci, F.F. Vincieri, B. Casetta, J. Agric. Food Chem. 43 (1995) 2104.

78. M.J. Herderich, P.A. Smith, Aust. J. Grape Wine Res. 11 (2005) 205.

79. H. Tamura, Y. Hayashi, H. Sugisawa, T. Kondo, Phytochem. Anal. 5 (1994) 190.

80. A. Marston, K. Hostettmann, in: Ø.M. Andersen, K.R. Markham (Eds), Flavonoids: Chemistry and Biochemistry and Applications, CRC Press,New York, (2006), p.1.

81. M.O. Downey, S. Rochfort, J. Chromatogr. A 1201 (2008) 43.

82. W.R. Melander, H.J. Lin, J. Jacobson, C. Horváth, J. Phys. Chem. 88 (1984) 4527.

83. J. Jacobson, W. Melander, G. Vaisnys, C, Horváth, J. Phys. Chem. 88 (1984) 4536.

84. J. Rigaud, M.T. Escribano-Bailon, C. Prieur, J-M. Souquet, V. Cheynier, J. Chromatogr. A 654 (1993) 255.

85. E. Hebrero, C. Santos-Buelga, J.C. Rivas-Gonzalo, Am. J. Enol. Vitic. 39 (1988) 227.

86. K. R. Maatta-Riihinen, A. Kamal-Eldin, P.H. Mattila, A.M. Gonzalez-Paramas, A.R. Torronen, J. Agric. Food Chem. 52 (2004) 4477.

87. V. Hong, R.E. Wrolstad, J. Agric. Food Chem. 38 (1990) 708.

88. A. de Villiers, G. Vanhoenacker, P. Majek, P. Sandra, J. Chromatogr. A 1054 (2004) 195.

89. I. Tarascou, J-P. Mazauric, E. Meudec, J-M. Souquet, D. Cunningham, S. Nojeim, V. Cheynier, H. Fulcrand, Food Chem. 128 (2011) 802. 
90. M.D. Hossain, D.K. Rai, N.P. Brunton, A.B. Martin-Diana, C. Barry-Ryan, J. Agric. Food Chem. 58 (2010) 10576.

91. J.S. Barnes, H.P. Nguyen, S. Shen, K. A. Schug, J. Chromatogr. A 1216 (2009) 4728.

92. Z. Huang, B. Wang, P, Williams, R.D. Pace, LWT-Food Sci. Technol. 42 (2009) 819.

93. B. Abad-García, L.A. Berrueta, S. Garmón-Lobato, B. Gallo, F. Vicente, J. Chromatogr. A 1216 (2009) 5398.

94. F. Sanchez-Rababeda, O. Jauregui, R.M. Lamuela-Raventos, F. Vilandomat, J. Bastida, C. Codina, Rapid Commun. Mass Spectrom. 18 (2004) 553.

95. X. Wu, R.L. Prior, J. Agric. Food Chem. 53 (2005) 3101.

96. X. Wu, R.L. Prior, J. Agric. Food Chem. 53 (2005) 2589.

97. S. Pati, I. Losito, G. Gambacorta, E. La Notte, F. Palmisano, P.G. Zambonin, J. Mass Spectrom. 41 (2006) 861.

98. Y. Li, R. Ma, Z. Xu, J. Wang, T. Chen, F. Chen, Z. Wang, J. Sci. Food Agric. 93 (2013) 1404.

99. A.A. De la Cruz, G. Hilbert, C. Riviere, V. Mengin, N. Ollat, L. Bordenave, S. Decroocq, J-C. Delaunay, S. Delrot, J-M. Merillon, J-P. Monti, E. Gomez, T. Richard, Anal. Chim. Acta. 732 (2012) 145.

100. M.B. Sanchez-Ilarduya, C. Sanchez-Fernandez, M. Viloria-Bernal, D.M. Lopez-Marquez, L.A. Berrueta, B. Gallo, F. Vicente, Aust. J. Grape Wine Res. 18 (2012) 203.

101. R. Flamini, Mass Spectrom. Rev. 22 (2003) 218.

102. P. Mazzuca, P. Ferranti, G. Picariello, L. Chianese, F. Addeo, J. Mass Spectrom. 40 (2005) 83.

103. H. Fulcrand, T. Doco, N.E. Es-Safi, V. Cheynier, M. Moutounet, J. Chromatogr. A 752 (1996) 85.

104. J.E. McNair, K.C. Lewis, J.W. Jorgenson, Anal. Chem. 69 (1997) 983.

105. D.C. Harris, Quantitative Chemical Analysis (Eds), W.H. Freeman and Co. New York, 1999.

106. A. de Villiers, F. Lestremau, R. Szucs, S. Gelebart, F. David, P. Sandra, J. Chromatogr. A 1127 (2006) 60.

107. T.L. Chester, Anal. Chem. 85 (2013) 579.

108. Y. Li, P. Aggarwal, H.D. Tolley, M.L. Lee, in E Grushka, N. Grinberg (Editors). Advances in chromatography. CRC Press, Boca Raton, FL. (2012) pp. 237.

109. F. Lestremau, A. de Villiers, F. Lynen, A. Cooper, R. Szucs, P. Sandra, J. Chromatogr. A 1138 (2007) 120. 
110. J.C. Giddings, Anal. Chem. 39 (1967) 1027.

111. R.E. Murphy, M.R. Schure, J.P. Foley, Anal. Chem. 70 (1998) 1585.

112. J.V. Seeley, J. Chromatogr. A. 962 (2002) 21.

113. K. Horie, H. Kimura, T. Ikegami, A. Iwatsuka, N. Saad, O. Fiehn, N. Tanaka, Anal. Chem. 79 (2007) 3764.

114. X. Li, R.D. Stoll, P.W. Carr, Anal. Chem. 81 (2009) 845.

115. J.C. Giddings, J. Chromatogr. A. 703 (1995) 3.

116. S.P. Dixon, I.D. Pitfield, D. Perrett, Biomed. Chromatogr. 20 (2006) 508.

117. P. Dugo, F. Cacciola, T. Kumm, G. Dugo, L. Mondello, J. Chromatogr. A 1184 (2008) 353.

118. M. Gilar, J. Fridrich, M.R. Schure, A. Jaworski, Anal. Chem. 84 (2012) 8722-8732.

119. G. Semard, V. Peulon-Agasse, A. Bruchet, J.P. Bouillon, P. Cardinaël, J. Chromatogr. A 1217 (2010) 5449.

120. S.C. Rutan, J.M. Davis, P.W. Carr, J. Chromatogr. A. 1255 (2012) 267.

121. J. M. Davis, D.R. Stoll, P.W. Carr, Anal. Chem. 80 (2008) 8122.

122. Z. Liu, D. G. Jr. Patterson, Anal. Chem. 67 (1995) 3840.

123. I. Francois, K. Sandra, P. Sandra Anal. Chim. Acta. 641 (2009) 14.

124. P.J. Schoenmakers, G. Vivo-Truyols, W. M. C. Decrop, J. Chromatogr. A 1120 (2006) 282.

125. A.M. van Nederkassel, A. Aerts, A. Dierick, D.L, Massart, Y. Vander Heyden, J. Pharm. Biomed. Analysis. 32 (2003) 233.

126. S. Eeltink, G. Desmet, G. Vivo-Truyols, G.P. Rozing, P.J. Schoenmaker, W.Th. Kok, J. Chromatogr. A 1104 (2006) 256.

127. K.M. Kalili, A. de Villiers, J. Chromatogr. A 1289 (2013) 58.

128. F. Bedani, P.J. Schoenmakers, H.-G. Janssen., J. Sep. Sci. 35 (2012) 1697.

129. A.R. Shalliker, M.J. Gray, in E. Grushka, N. Grinberg (Editors), Advances in Chromatography, CRC Press, New York, (2006) pp.177.

130. K.M. Kalili, A. de Villiers, J. Chromatogr. A 1289 (2013) 69.

131. M.R. Filgueira, Y. Huang, K. Witt, C. Castells, P.W. Carr, Anal. Chem. 83 (2011) 9531.

132. D.R. Stoll, J.D. Cohen, P.W. Carr, J. Chromatogr. A 1122 (2006) 123.

133. M. Hehn, K. Maiko, H. Pasch, W. Hiller, Macromol. 46 (2013) 7678.

134. P. Jandera, J. Fischer, H. Lahovska, K. Novotna, C. Cesla, L. Kolarova, J. Chromatogr. A 1119 (2006) 3.

135. A. van der Horst, P.J. Schoenmakers, J. Chromatogr. A 1000 (2003) 693. 
136. M. Maiko, M. Hehn, W. Hiller, H. Pasch, Anal. Chem. 85 (2013) 9793.

137. K.M. Kalili, J. Vestner, M.A. Stander, A. de Villiers, Anal. Chem. 85 (2013) 9107.

138. T. Beelders, K.M. Kalili, E. Joubert, D. de Beer, A. de Villiers, J. Sep. Sci. 35 (2012) 1808.

139. G.J. Opiteck, J.W. Jorgenson, Anal. Chem. 69 (1997) 2283.

140. D.R. Stoll, P.W. Carr, J. Am. Chem. Soc. 127 (2005) 5034.

141. P. Dugo, N. Fawzy, F. Cichello, F. Cacciola, P. Donato, L. Mondello, J. Chromatogr. A 278 (2013) 46.

142. G.J. Opiteck, S.M. Ramirez, J.W. Jorgenson, A.M. Moseley III, Anal. Biochem. 258 (1998) 349.

143. P.Q. Tranchida, P. Dugo, G. Dugo, L. Mondello, J. Chromatogr. A 1054 (2004) 3.

144. P. Dugo, F. Cacciola, P. Donato, D. Airado-Rodríguez, M. Herrero, L. Mondello, J. Chromatogr. A. 1216 (2009) 7483.

145. M. Kivilompolo, T. Hyotylainen, J. Sep. Sci. 31 (2008) 3466

146. I. Francois, A. de Villiers, B. Tienpont, F. David, P. Sandra, J. Chromatogr. A. 1178 (2008) 33.

147. P. Dugo, O. Favoino, R. Luppino, G. Dugo, L. Mondello, Anal. Chem. 76 (2004) 2525.

148. P. Dugo, V. Skerikova, T. Kumm, A. Trozzi, P. Jandera, L. Mondello, Anal. Chem. 78 (2006) 7743.

149. K.M. Kalili, A. de Villiers, J. Sep. Sci. 33 (2010) 853.

150. K.M. Kalili, A. de Villiers, J. Chromatogr. A 1216 (2009) 6274.

151. F. Cacciola, P. Jandera, E. Blahova, L. Mondello, J. Sep. Sci. 29 (2006) 2500.

152. F. Cacciola, P. Jandera, 1. Mondello, J. Sep. Sci. 30 (2007) 462.

153. F. Cacciola, P. Jandera, Z. Hajdu, P. Cesla, L. Mondello, J. Chromatogr. A 1149 (2007) 73.

154. M. Kivilompolo, T. Hyotylainen, J. Chromatogr. A 1145 (2007) 155.

155. P. Cesla, T. Hajek, P. Jandera, J. Chromatogr. A. 1216 (2009) 3443.

156. P. Dugo, F. Cacciola, M. Herrero, P. Donato, L. Mondello, J. Sep. Sci. 31 (2008) 3297.

157. I. Francois, A. de Villiers, P. Sandra, J. Sep. Sci. 29 (2006) 492.

158. M. R. Filqueira, Y. Huang, K. Witt, C. Castells, P.W. Carr, Anal. Chem. 83 (2011) 9531.

159. P. Jandera, T. Hájek, M. Stanková, K. Vynuchalová, P. Cesla, J. Chromatogr. A. 1268 (2012) 91.

160. L. Montero, M. Herrero, E. Ibanez, A. Cifuentes., J. Chromatogr. A 1313 (2013) 275.

161. E. Blahova, P. Jandera, F. Cacciola, L. Mondello., J. Sep. Sci. 29 (2006) 555. 
162. E. P. Toups, M. J. Gray, G. R. Dennis, N. Reddy, M. A. Wilson, R. A. Shalliker, J. Sep. Sci. 29 (2006) 481

163. C.M. Willemse, M.A. Stander, A.G.J. Tredoux, A. de Villiers, J. Chromatogr. A 1359 (2014) 189. 


\section{Chapter 3}

\section{Results and Discussion}




\subsection{Introduction}

The aim of this dissertation was to develop comprehensive two-dimensional liquid chromatographic ( $\mathrm{LC} \times \mathrm{LC})$ methods for anthocyanins and their derived products in red wine. Several key requirements had to be fulfilled in order to achieve this. First, a hydrophilic interaction chromatographic (HILIC) method was developed and evaluated as a complementary separation mode to reversed-phase liquid chromatography (RP-LC) for the analysis of anthocyanins. The developed HILIC method was used in the first dimension in LC $\times$ LC analyses, first of all through the off-line coupling to RP-LC. Optimal experimental conditions were established for both first and second dimensions to provide exceptionally high practical peak capacities, although at the cost of very long analysis times. This information was then used for the development and optimisation of an on-line HILIC $\times$ RP-LC separation using a capillary LC system in the first dimension and an Ultra-performance liquid chromatographic (UPLC) system in the second. The on-line HILIC $\times$ RP-LC method was hyphenated to a high resolution mass spectrometer for the identification of a wide range of anthocyanin derived pigments in red wine.

\subsection{Hydrophilic interaction chromatographic analysis of anthocyanins}

C.M. Willemse, M.A. Stander, A. de Villiers, J. Chromatogr. A 1319 (2013) 127-140; Addendum A.

RP-LC is the separation method of choice for the analysis of anthocyanins due to its high selectivity and good chromatographic performance. RP-LC is usually coupled to a photo-diode array detector (PDA) which has the advantage of selective detection of these analytes at 500 $\mathrm{nm}$, allowing them to be distinguished from other flavonoid classes [1,2]. When hyphenated to mass spectrometry (MS) additional structural information (i.e., molecular mass and fragments) can be obtained for compound identification. However, despite these advantages RP-LC-MS suffers from some limitations, and these, in combination with the complexity of anthocyanins, means that their complete characterisation is often not possible using a single method. For these reasons, alternative separation methods remain relevant in anthocyanin analysis. HILIC would be a promising alternative mode of separation to RP-LC because of its complementary selectivity and suitability for polar molecules. This technique has found widespread application 
for the analysis of various phenolic compounds, and also shows promise for hyphenation to other modes of HPLC in 2-dimensional LC, as has been demonstrated for the analysis of phenolic compounds by various researchers [3-7]. The aim of this work was therefore to examine the suitability of HILIC for the analysis of anthocyanins in natural products. To this end, diverse anthocyanins found in blueberries, black beans, red grape skins, red radish and red cabbage were used as target analytes.

In the development of the HILIC method for anthocyanins, several experimental parameters were systematically optimised. First, a range of HILIC stationary phases were evaluated using a scouting gradient, to determine which was most suitable for the target analytes. Of the six different stationary phases evaluated, the best results in terms of retention and selectivity were obtained on a BEH amide stationary phase. Even on this column though, broad peaks were obtained for the anthocyanins in all samples using the scouting gradient. The exception was the red radish anthocyanins, for which good efficiency was attributed to the fact that this sample uniquely contained acylated pelaragonidin derivatives. This then lead to the optimisation of the mobile phase composition to improve chromatographic performance. Since anthocyanins exist as different chemical species in solution, the mobile phase $\mathrm{pH}$ has a profound effect on their solution chemistry [8-10]. For the RP-LC analysis of anthocyanins, for example, low pH mobile phases are essential to ensure prevalence of the flavylium cationic species, thereby improving the chromatographic performance by minimising the detrimental effects of secondary equilibria $[11,12]$.

Under the chromatographic conditions used for the scouting gradient (5\% formic acid in acetonitrile and water), the corrected $\mathrm{pH}$ was determined to be 2.20 . At this $\mathrm{pH}$, a significant portion of anthocyanins are present in the carbinol pseudobase form, and the resulting interconversion between flavylium cationic and carbinol species is expected to cause severe peak broadening [11]. Decreasing the $\mathrm{pH}$ of the mobile phase by increasing the formic acid content to $10 \%$ confirmed this conclusion: improved peak shapes, selectivity and resolution were obtained, proving that mobile phase $\mathrm{pH}$ also plays an important role in HILIC. Trifluoroacetic acid (TFA, $0.4 \%$ ) was also investigated as an alternative acidic mobile phase component and was found to further improve peak shapes and increase retention due to the lower polarity of this mobile phase. The effect of temperature was investigated, since an increase in temperature has been shown to improve the chromatographic separation of anthocyanins due to faster interconversion between anthocyanin species [11]. Indeed, much better performance was observed 
at $50^{\circ} \mathrm{C}$. Finally, the optimal mobile phase flow rate was established by measuring the plate height curve for malvidin-3-O-glucoside under the optimal conditions.

The optimal method was hyphenated to a high resolution time-of-flight (TOF) MS detector operated in positive ionisation mode for the identification of the anthocyanins in each of the analysed samples. Tentative identification was based on accurate mass data, fragmentation information in $\mathrm{MS}^{\mathrm{E}}$ mode and comparison of UV- and MS spectra with previous literature reports. Anthocyanidin bases were detected as base peaks in the $\mathrm{MS}^{\mathrm{E}}$ spectra, following the loss of $m / z, 162$ or 132 (monoglucosides), m/z 204 (acetyl-glucosides), m/z 308 (coumaroylglucosides) and $m / z 324$ (diglucoside or caffeoyl-glucoside), etc. Furthermore, fragmentation of the anthocyanidin base under high collision induced dissociation (CID) $\mathrm{MS}^{\mathrm{E}}$ conditions (20 - $60 \mathrm{~V}$ ) was observed, which allowed confirmation of the anthocyanidin moiety $[1,13]$.

A total of 35 anthocyanins were identified in black beans, blueberries and grape skins, comprising mainly of monoglucosides in black beans and blueberries and additional acylated mono-glucosides in grape skins. Anthocyanidin-galactosides could not be distinguished from the corresponding -glucosides due to co-elution in HILIC. Interestingly, several anthocyanin derived pigments were also detected in grape skins, of which acetylvitisin A, coumaroylvitisin A and the B-type vitisins were reported in grape skins for the first time.

Red cabbage and red radish contain highly acylated anthocyanins; 36 compounds were tentatively identified in these samples. The major anthocyanidin found in red cabbage was cyanidin, with variations stemming from different acylation patterns of cyanidin-3di/triglucosides. It was not possible to unambiguously identify several compounds with identical molecular weights and fragmentation patterns based on HILIC retention and MS data alone. In red radish, only pelaragonidin derivatives were detected, with the exception of cyanidin-3-(glycosyl)rhamnoside and cyanidin-3-(diglycosyl)rhamnoside (the latter identified for the first time in this sample). Several isomeric structures which could not unambiguously be distinguished were also detected in red radish. Pelaragonidin-(feruloyl)triglucoside-5(malonoyl)glucoside and pelaragonidin-3-(p-coumaroyl)triglucoside-5-(malonoyl)glucoside were tentatively identified for the first time in red radish.

Since this work represented the first application of HILIC for anthocyanin analysis, it was interesting to investigate the retention behaviour of these compounds in this mode. It was found that, for the same glycosylation and acylation pattern, compounds were separated according to the polarity of the anthocyanidin base. Anthocyanidins containing the most methoxy- 
substituents, i.e., malvidin derivatives, eluted first, followed by peonidin, petunidin, pelaragonidin, cyanidin and lastly delphinidin derivatives. Furthermore, anthocyanidinarabinosides eluted before the corresponding -hexosides (the opposite is true for RP-LC), and anthocyanidin-glucosides co-eluted with the corresponding -galactosides in HILIC. Retention in HILIC increases with the degree of glycosylation and decreases with acylation of anthocyanidins, the exception being caffeoyl-derivatives which show higher retention than the corresponding non-acylated anthocyanidins. Comparison of HILIC retention behaviour with the well-established RP-LC elution characteristics of anthocyanins therefore highlights the complementary nature of the two separation modes.

\subsection{Comprehensive two-dimensional liquid chromatographic analysis of anthocyanins}

C.M. Willemse, M.A. Stander, A.G.J. Tredoux, A. de Villiers, J. Chromatogr. A 1359 (2014) 189-201; Addendum B

The coupling of two independent LC separations in comprehensive two-dimensional liquid chromatography (LCXLC) offers a dynamic and powerful approach for the separation of compounds in complex matrices. In this work the previously developed HILIC method was combined with RP-LC for the LC $\times$ LC analysis of anthocyanins. Off-line HILIC $\times$ RP-LC was chosen for the separation of these compounds, since this mode of hyphenation is the easiest to implement and places no restrictions on the second dimension analysis time. This factor is especially important for anthocyanin analysis, since the short second dimension analysis times required for on-line $\mathrm{LC} \times \mathrm{LC}$ are especially detrimental for these compounds due to the effect of secondary equilibria [11,12]. In addition, off-line operation provides the highest overall practical peak capacity.

Systematic optimisation of the off-line HILIC $\times$ RP-LC method involved first of all the choice of first and second dimension column dimensions, flow rates and gradient times. The previously developed HILIC method [14] was adapted to a wide-bore $150 \times 4.6 \mathrm{~mm}$ i.d. column operated at a flow rate of $0.2 \mathrm{~mL} / \mathrm{min}$. These conditions were selected based on the low optimal flow rate for anthocyanins in HILIC [14]: for off-line LC $\times$ LC using short sampling times, this flow rate provided ideal fraction volumes. This column also provided a higher sample capacity and peak capacity, the latter due to the reduced effect of extra-column peak broadening. A 
superficially porous $\mathrm{C} 18$ column $(50 \times 4.6 \mathrm{~mm}$ i.d., $2.6 \mu \mathrm{m})$ was used in the second dimension, with a RP-LC gradient method based on that of de Villiers et al [11, 12] for the high-efficiency separation of anthocyanins. A second dimension gradient time of 30 minutes was used.

One of the major challenges coupling HILIC and RP-LC is the relative elution strengths of the solvents used in the two dimensions [15-17]. A maximum of $2 \mu \mathrm{L}$ of each HILIC fraction could be injected onto the second dimension column to avoid peak distortion of the early eluting compounds (this was confirmed experimentally). A sampling time, ${ }^{1} t_{s}$, of 0.5 minutes was selected, which provided an acceptable degree of under-sampling of the first dimension peaks. Fraction volumes of $100 \mu \mathrm{L}$ were therefore collected (flow rate $0.2 \mathrm{~mL} / \mathrm{min}$ ), meaning that $\sim 2 \%(2 \mu \mathrm{L})$ of each HILIC fraction was injected onto the RP column.

Raw UV data at $500 \mathrm{~nm}$ were used to construct contour plots of each of the analysed samples, and compounds were identified based on 1-dimensional HILIC- and RP-LC-ESI-TOF-MS data. A total of 87 anthocyanins were identified in the contour plots for blueberries, black beans, grape skins, red cabbage and red radish samples. The contour plots revealed improved resolution for numerous compounds that could not be separated in one of the 1-dimensional separations. Furthermore, it was noted that group-type separation of anthocyanins was afforded by the combination of HILIC and RP-LC separations. This was especially evident for the highly acylated and glycosylated anthocyanins identified in red grape skins and red cabbage. The position of compounds in these contour plots thereby provides additional information that could assist in compound identification.

The performance of the off-line HILIC $\times$ RP-LC separation was evaluated in terms of peak capacity, $n_{c}[18,19]$. The 1 -dimensional HILIC separations provided peak capacities ranging between 46 and 78 for the different samples, whereas RP-LC provided peak capacities ranging between 109 and 196 (average 163). This exceptional performance in RP-LC can be ascribed to the use of a $2.6 \mu \mathrm{m}$ superficially porous column operated at optimal flow rate and elevated temperature[11,12]. In order to obtain a realistic measure of the performance of HILIC $\times$ RPLC separation of anthocyanins, practical 2-dimensional peak capacities $\left(n_{c, 2 D}^{*}\right)$ were calculated taking first dimension under-sampling and finite orthogonality into account according to:

$$
n_{c, 2 D}^{*}=\frac{{ }^{1} n_{c} \times{ }^{2} n_{c} \times f_{c}}{\beta}
$$

\section{Equation 3.1}


where, $\beta$ is a measure of first dimension under-sampling, calculated according to Li et al [20], and $f_{c}$ is fractional surface coverage of the available 2-dimensional separation space [21]. Two methods were used to estimate the effective surface coverage (SC): the 'bin counting' method of Stoll et al [22] and the convex hull method [23, 24]. Differences in SC values between these methods were noted, which emphasises the importance of the metrics used to estimate orthogonality in the calculation of practical 2-D peak capacity. Practical peak capacities of up to 6950 were obtained for the off-line HILIC $\times$ RP-LC analysis of anthocyanins, providing peak production rates up to 2.5 peaks/min. It should be noted that this gain in performance compared to 1-dimensional LC methods is only attainable using off-line LC $\times \mathrm{LC}$, and therefore very long analysis times, since a longer second dimension gradient time can be used to provide high peak capacities in this dimension [25]. Nonetheless, HILIC $\times$ RP-LC was found to offer a promising method for anthocyanin analysis, with the group-type separation obtained by the combination of these modes especially useful in facilitating compound identification.

\subsection{Comprehensive two-dimensional HILIC $\times$ RP-LC-UV-MS analysis of anthocyanins and derived pigments in wine}

C.M. Willemse, M.A. Stander, J. Vestner, A.G.J. Tredoux, A. de Villiers, (submitted to Anal. Chem.); Addendum C

The final experimental work involved the on-line comprehensive HILIC $\times$ RP-LC-MS analysis of anthocyanins and derived pigments in red wine. Despite the important roles that anthocyanins play in determining many important wine characteristics, the accurate qualitative and quantitative analysis of wine pigments remains challenging due to their extreme structural diversity and complexity. During wine ageing, the grape-derived anthocyanins are transformed through a range of chemical reactions involving other wine constituents to a large number of derived pigments, which are responsible for the evolution and stabilisation of wine colour as well as changes in the sensory properties of the product. Because of the complexity of the derived products and their low levels in aged wines, MS plays a critical role in their identification. Therefore, in this work the development of an on-line HILIC $\times$ RP-LC method and its hyphenation to high resolution time-of-flight mass spectrometry (HR-Q-TOF-MS) was explored for the detailed analysis of anthocyanins and derived products in red wine. 
For the development of the on-line HILIC $\times$ RP-LC method, the performance of each 1dimensional separation mode was evaluated under various conditions to enable the selection of the best conditions for the on-line method. The first dimension the flow rate $\left({ }^{1} F\right)$ and gradient time were varied using a $1 \mathrm{~mm}$ i.d. amide column operated at $50^{\circ} \mathrm{C}$, and the resulting peak width measured and used to calculate the peak capacity according to:

$n_{c, 1 D}=1+\frac{t_{g}}{1 / n \sum_{1}^{n} w_{b}}$

equation 3.2

where $t_{g}$ is the gradient time and $w_{b}$ is the peak width at baseline, averaged for $n$ peaks. In the second dimension the flow rate and column temperature remained constant at $860 \mu \mathrm{L} / \mathrm{min}$ and $60^{\circ}$, respectively, and the second dimension cycle time $\left({ }^{2} t_{c}\right)$ was varied. RP-LC peak capacities were calculated according to equation 3.1 for each cycle time, and these were fit to an empirical equation [26] which allowed determination of ${ }^{2} n_{c}$ for any given ${ }^{2} t_{c}$. For each set of conditions, the practical LCXLC peak capacity was calculated according to equation 3.2 by correcting for limited orthogonality using the surface coverage metric determined by the convex hull method and first dimension under-sampling using $\beta$, calculated using first dimension peak widths. To accommodate the fact that the maximum injection volume of HILIC fractions in the second dimension is $2 \mu \mathrm{L}$, flow splitting after the first dimension column was assumed in these calculations. The results of this optimisation process showed that the highest 2-dimensional peak capacity would be obtained at low first dimension flow rates and long first dimension gradients. At the optimal conditions $\left({ }^{1} F=1 \mu \mathrm{L} / \mathrm{min},{ }^{1} t_{g}=416 \mathrm{~min},{ }^{1} t_{s}=10 \mathrm{~min}\right)$ a split flow of $4: 1$ would be required, providing a practical peak capacity of 2173 . However, because of the low levels of pigments in especially aged wine, we opted to use a first dimension flow rate of $1 \mu \mathrm{L} / \mathrm{min}$ and a sampling time of $2 \mathrm{~min}$, thereby avoiding the need to split at the cost of some performance loss.

Under these conditions, the first dimension separation was performed on a Cap-LC system, which was hyphenated through a high-pressure 10-port 2-position valve to a UPLC instrument where RP-LC separation was performed using a cycle time of 2 min. A UV detector acquired data on-line just after the second dimension column, where after the effluent was split $~ 1: 1$ before connection to the Q-TOF-MS detector. Raw MS and UV data at $500 \mathrm{~nm}$ were exported to Matlab 7.14 for data processing.

The samples used in this study were one- and six-year old Pinotage wines, pre-concentrated using an in-house developed solid phase extraction (SPE) procedure. The obtained contour 
plots illustrate the complexity of pigmented fractions of especially aged red wines. Both grapederived anthocyanins and a large number of their derived pigments were tentatively identified using accurate mass $\mathrm{MS}$ and $\mathrm{MS}^{\mathrm{E}}$ data. In total, 94 pigments were identified, including anthocyanidin-mono- and -di-glucosides, oligomeric anthocyanins, anthocyanin-tannin adducts, acetaldehyde-mediated tannin adducts, vinylflavanol condensation products, oxovitisins, A and B-type vitisins and anthocyanin-vinylcatechol, -vinylphenol, -vinylguaicol and-vinylsyringol derivatives. The differences in pigment composition between the two wines were clearly evident from the contour plots. For example the 2013 wine contained more grapederived anthocyanins such as anthocyanidin-mono- and di-glucosides as well as oligomeric anthocyanins, whereas levels of anthocyanin-tannin adducts, vinylflavanols, A-type vitisins, vinylcatechol, -guaicol and -syringol derivatives were higher in the older wine.

The benefit of using HILIC $\times$ RP-LC-MS is clearly evident from the contour plots, as neither HILIC nor RP-LC provides complete resolution of all compounds, especially using a 2 min second dimension gradient. For example, 1-dimensional HILIC-MS cannot distinguish between isomeric compounds such as anthocyanidin-galactosides and glucosides or cis and trans isomers, whereas RP-LC provides separation of these isomers based on hydrophobicity. In addition, the structured elution pattern offered by HILIC $\times$ RP-LC separation provides an added advantage in that it allows the tentative assignment of the degree and/or nature of glycosylation and/or acylation of unidentified peaks according to their position in the contour plots relative to known compounds.

The results presented in this work illustrate that HILIC $\times$ RP-LC hyphenated to high resolution MS offers a powerful analytical method for the detailed analysis of anthocyanins and derived pigments in wine. This methodology shows promise for the study of wine pigment evolution, and potentially for elucidation of new reactions involving anthocyanins during wine ageing. 


\subsection{References}

1. P. Alberts, M.A. Stander, A. de Villiers, J. Chromatogr. A 1235 (2012) 92.

2. A. de Villiers, G. Vanhoenacker, P. Majek, P. Sandra, J. Chromatogr. A 1054 (2004) 195.

3. T. Beelders, K.M. Kalili, E. Joubert, D. de Beer, A. de Villiers, J. Sep. Sci. 35 (2012) 1808.

4. K.M. Kalili, A. de Villiers, J. Chromatogr. A 1216 (2009) 6274.

5. K.M. Kalili, A. de Villiers, J. Sep. Sci. 33 (2010) 853.

6. K.M. Kalili, A. de Villiers, J. Chromatogr. A 1289 (2013) 69.

7. K.M. Kalili, J. Vestner, M.A. Stander, A. de Villiers, Anal. Chem. 85 (2013) 9107.

8. R. Brouillard, J.E. Dubois, J. Am. Chem. Soc. 99 (1976) 1359.

9. R. Brouillard, B. Delaporte, J. Am. Chem. Soc. 99 (1977) 8461.

10. R. Brouillard, J.-E. Dubois, J. Am. Chem. Soc. 99 (1977) 1359.

11. A. de Villiers, D. Cabooter, F. Lynen, G. Desmet, P. Sandra, J. Chromatogr. A 1216 (2009) 3270.

12. A. de Villiers, D. Cabooter, F. Lynen, G. Desmet, P. Sandra, J. Chromatogr. A 1218 (2011) 4660.

13. B. Abad-García, L.A. Berrueta, S. Garmón-Lobato, B. Gallo, F. Vicente, J. Chromatogr. A 1216 (2009) 5398.

14. C.M. Willemse, M.A. Stander, A. de Villiers, J. Chromatogr. A 1319 (2013) 127.

15. P.J. Schoenmakers, G. Vivo-Truyols, W.M.C. Decrop, J. Chromatogr. A 1120 (2006) 282.

16. P. Dugo, F. Cacciola, T. Kumm, G. Dugo, L. Mondello, J. Chromatogr. A 1184 (2008) 353.

17. P. Jandera, T. Hájek, M. Stanková, K. Vynuchalová, P. Cesla, J. Chromatogr. A 1268 (2012) 91.

18. J.C. Giddings, Anal. Chem. 39 (1967) 1027.

19. J. C. Giddings, Unified Separation Science, Ed, John Wiley \& Sons, Inc. New York, 1991.

20. X. Li, R.D. Stoll, P.W. Carr, Anal. Chem. 81 (2009) 845.

21. M. Gilar, J. Fridrich, M.R. Schure, A. Jaworski, Anal. Chem. 84 (2012) 8722-8732.

22. J.M. Davis, D.R. Stoll, P.W. Carr, Anal. Chem. 80 (2008) 8122.

23. S. C. Rutan, J. M. Davis, P.W. Carr., J. Chromatogr. A 1255 (2012) 267.

24. G. Semard, V. Peulon-Agasse, A. Bruchet, J.P. Bouillon, P. Cardinaël, J. Chromatogr. A 1217 (2010) 5449. 
25. Z. Liang, K. Li, X. Wang, Y. Ke, Y. Jin, X. Liang, J. Chromatogr. A 1224 (2012) 61.

26. J.N. Fairchild, K. Horváth, G. Guiochon, J. Chromatogr. A 1216 (2009 ) 1363. 


\section{Chapter 4}

\section{General Conclusions and}

\section{Recommendations}




\subsection{General conclusions}

Anthocyanins are important natural pigments, whose analysis remains challenging due their unique solution properties, structural diversity and the lack of commercially available standards. This is especially the case for the grape-derived anthocyanins and their derived pigments found in red wine due to the low levels and large number of related derivatives formed during wine ageing. The ultimate goal of the research reported in this thesis was therefore to develop improved analytical methods for the determination of the complex mixtures of anthocyanins, with special emphasis on the pigments encountered in red wine. Since a single liquid chromatographic method is not capable of resolving such large numbers of related compounds, the combination of multiple orthogonal separations in comprehensive 2dimensional liquid chromatography $(\mathrm{LC} \times \mathrm{LC})$ was explored for this purpose.

In order to achieve the goal of improved separation of anthocyanins by LC $\times \mathrm{LC}$, a systematic approach was followed:

i. In the first instance, a hydrophilic interaction chromatography (HILIC) method was developed as an alternative separation mode to reversed-phase liquid chromatography (RP-LC) for the analysis of anthocyanins. Method development involved optimisation of several key parameters, such as the stationary- and mobile phases, column temperature and flow rate, to provide optimal chromatographic performance. The optimised HILIC method was hyphenated to selective ultra-violet (UV) detection at $500 \mathrm{~nm}$ and high resolution mass spectrometry (HR-MS) for identification purposes. The potential of the HILIC-UV-MS method was demonstrated by its application to a wide range of anthocyanins, ranging from relatively simple to highly complex in terms of structure, in blueberries, grape skins, red cabbage, red radish and black beans. The developed method provided and alternative separation selectivity compared to RP-LC and was therefore suitable for coupling to RP-LC in LC $\times \mathrm{LC}$.

ii. The optimised HILIC method was coupled to RP-LC in an off-line LC $\times$ LC configuration. Each 1-dimensional method was optimised prior to hyphenation, which involved selection of column dimensions and flow rates in each dimension as well as optimal sampling times $\left({ }^{1} t_{s}\right)$ and second dimension cycle times $\left({ }^{2} t_{c}\right)$ for maximum resolution. The applicability of off-line HILIC $\times$ RP-LC in combination with UV and MS detection was demonstrated for the same samples and compounds as in (i). Off-line HILIC $\times$ RP-LC showed improved separation of compounds not resolved in either 
HILIC or RP-LC. Exceptionally high practical peak capacities were calculated for the off-line HILIC $\times$ RP-LC separation. Also of interest was that group-type separation of anthocyanins was observed in the contour plots.

iii. In the final part of the work, an on-line HILIC $\times$ RP-LC method hyphenated to high resolution time-of-flight mass spectrometry (HR-Q-TOF-MS) was developed for the analysis of anthocyanins and derived pigments in old and young red wines. For the online method, optimisation of each 1-D method and the parameters used in their coupling was performed. For the first dimension separation, a $1 \mathrm{~mm}$ i.d. column was used at a flow rate of $1 \mu \mathrm{L} / \mathrm{min}$ and various gradient times and flow rates were evaluated, while in the second dimension various second dimension cycle times were evaluated on a 50 $\mathrm{mm} 1.7 \mu \mathrm{m}$ superficially porous column. By using a sampling time of $2 \mathrm{~min}$, the need to split the first dimension eluent before the second dimension column was avoided. A large number of pigments were identified in red wine by HILIC $\times$ RP-LC-MS, including anthocyanidin-mono- and -di-glucosides, oligomeric anthocyanins, anthocyanin-tannin adducts, acetaldehyde-mediated tannin adducts, vinylflavanol condensation products, oxovitisins, A and B-type vitisins and anthocyanin-vinylcatechol, -vinylphenol, vinylguaicol and -vinylsyringol derivatives. Differences in pigment composition between the two wines were clearly evident from the contour plots. For example the 2013 wine contained more grape-derived anthocyanins such as anthocyanidin-monoand di-glucosides and oligomeric anthocyanins, whereas levels of anthocyanin-tannin adducts, vinylflavanols, A-type vitisins, vinylcatechol, -guaicol and -syringol derivatives were higher in the older wine.

Based on the results obtained in this study for various samples and analytes, it is clear that the combination of multiple complementary analytical techniques is highly beneficial for anthocyanin determination, since their complexity in several natural products exceeds the separation capabilities of a single analytical technique. One of the benefits of performing LC $\times$ LC clearly illustrated in this work, is that compounds co-eluting in one dimension were separated in the other, and vice versa.

Both on-line and off-line HILIC $\times$ RP-LC methods were developed. In both cases, optimal performance required careful optimisation of certain parameters, including gradient times, sampling times, flow rates and column dimensions. On-line as well as off-line LC $\times$ LC offered 
improved separation performance compared to 1-dimensional HILIC and RP-LC. On-line HILIC $\times$ RP-LC offers the advantage of automation and conventional analysis times, whereas off-line operation provides higher peak capacities at longer analysis times.

Hyphenation of HILIC $\times$ RP-LC with UV and MS in an on-line manner provided not only a third dimension but also an increase in specificity. In particular, on-line HILIC $\times$ RP-LC-UVMS offered a powerful method for screening of wine pigments. Even though complete separation was not provided, group-type retention information and the power of high resolution MS allow the potential identification of new analytes and elucidation of their formation during wine ageing.

In conclusion, one-dimensional HILIC and both off-line and on-line HILIC $\times$ RP-LC methods were developed for anthocyanin analysis for the first time. Hyphenation of HILIC $\times$ RP-LC separation to HR-TOF-MS was shown to be a powerful method for the detailed characterisation of anthocyanins and derived pigments in highly complex samples such as wine, and this approach shows promise for shedding further light on these influential natural pigments.

\subsection{Recommendations for future studies}

Due to the complexity associated with anthocyanins and their derived pigments in wine and the potential of HILIC $\times$ RP-LC for their analysis as demonstrated in this dissertation, further research is required to improve these methods or extend already developed methods. Firstly, due to the low levels of these pigments in especially aged wine, selective extraction and preconcentration of these pigments should be performed to improve the sensitivity of the methods used here and allow identification of more trace-level compounds.

Since stop-flow $\mathrm{LC} \times \mathrm{LC}$ is considered an automated alternative to off-line $\mathrm{LC} \times \mathrm{LC}$, it would be interesting to combine HILIC and RP-LC separations under stop-flow conditions. To do this, the effective diffusion coefficients should be measured, since additional first-dimension peakbroadening might be the limiting factor.

Because MS cannot distinguish between isomeric anthocyanins and their derivatives, several of which were detected in wine, it would be relevant extend HILIC $\times$ RP-LC separation to the preparative level to isolate particular compounds for structural elucidation by nuclear magnetic 
resonance (NMR) spectroscopy. Hyphenation of NMR to HILIC $\times$ RP-LC would of course also be an option, although much more complicated in practice.

In order to enhance the orthogonality of the HILIC $\times$ RP-LC separation of anthocyanins, it would be interesting to evaluate the use of different gradient profiles in the second dimension. The use of segmented in fraction (SIF), full in fraction (FIF), or continuous shifting (CF) gradients may prove useful in this regard (P. Jandera et al, J. Sep. Sci. (2010) 33: 1382-1397, G. M. Leme et al, Anal. Bioanal. Chem. (2014) 406: 4315-4324).

Extension of the developed methodologies to other anthocyanin-containing natural products such as cranberries and pomegranate would be interesting, since these samples contain mixtures of different pigments. Finally, exploring the simultaneous analysis of both anthocyanins and non-coloured flavonoids in samples such as wine, cranberries, etc, would extend the applicability of the methods developed in this thesis. 


\section{Addenda}

\section{Research Publications and}

Supporting Information 


\section{Addendum A}

\section{Hydrophilic interaction chromatographic analysis of anthocyanins}

C.M. Willemse, M.A. Stander, A. de Villiers, J. Chromatogr. A 1319 (2013) 127-140.

Author contributions: C.M.W. performed all experiments, did initial data analysis and wrote the first draft of the paper. M.A.S. and A.d.V. assisted with MS data analysis and edited the manuscript. 


\title{
Hydrophilic interaction chromatographic analysis of anthocyanins ${ }^{\text {is }}$
}

\author{
Chandré M. Willemse ${ }^{\mathrm{a}}$, Maria A. Stander ${ }^{\mathrm{b}, \mathrm{c}}$, André de Villiers ${ }^{\mathrm{a}, *}$ \\ a Stellenbosch University, Department of Chemistry and Polymer Science, Private Bag X1, Matieland 7602, South Africa \\ b Stellenbosch University, Central Analytical Facility, Private Bag X1, Matieland 7602, South Africa \\ c Stellenbosch University, Department of Biochemistry, Private Bag X1, Matieland 7602, South Africa
}

\section{A R T I C L E I N F O}

\section{Article history:}

Received 2 August 2013

Received in revised form 7 October 2013

Accepted 12 October 2013

Available online 20 October 2013

\section{Keywords:}

Anthocyanins

Hydrophilic interaction chromatography

(HILIC)

HPLC

Quadrupole-time-of-flight mass

spectrometry (Q-TOF-MS)

Phenolics

\begin{abstract}
A B S T R A C T
Hydrophilic interaction chromatography (HILIC) provides an alternative separation mode for the analysis of phenolic compounds, in which aqueous-organic mobile phases with polar stationary phases are used. This paper reports the evaluation of HILIC for the analysis of the natural pigments anthocyanins, which are of importance because of their chromophoric properties and a range of health benefits associated with their consumption. Several HILIC stationary phases (silica, diol, amine, cyanopropyl and amide) and mobile phase combinations were evaluated, with the latter proving particularly important due to the distinctive chromatographic behaviour of anthocyanins. Diode array detection was used for selective detection of anthocyanins, while high resolution quadrupole-time-of-flight mass spectrometry (Q-TOFMS) was used for compound identification. The potential of HILIC separation is demonstrated for a range of anthocyanins varying in glycosylation and acylation patterns found in blueberries, grape skins, black beans, red cabbage and red radish. HILIC is shown to be a complementary separation method to reversed phase liquid chromatography (RP-LC) due to the alternative retention mechanism.
\end{abstract}

(c) 2013 Elsevier B.V. All rights reserved.

\section{Introduction}

Extensive research has been focused on plants and food rich in phenolic antioxidants, specifically fruit and vegetables, since they play a pivotal role in the reduced risk of a number of chronic and degradative diseases in humans [1-3]. Fruits and vegetables are one of the most important sources of phenolic compounds in the human diet. The phenolic pigments anthocyanins (anthocyanidinglycosides), a class of the flavonoid family, are responsible for the red, blue and violet colours of many plants, flowers, fruits, grains and vegetables [4]. Anthocyanins have been used as natural food colourants for many years, but recently attention has shifted towards their possible application as nutritional supplements, constituents of functional food formulae, medicines, etc. [5]. Due to the importance of anthocyanins to the food industry, understanding their chemical structure and distribution is of some significance. Anthocyanins are predominantly found in nature as glycosides of polyhydroxy- or polymethoxy-derivatives of flavylium salts. The six common anthocyanidins found in nature are pelaragonidin (Pel), cyanidin (Cy), peonidin (Pe), delphinidin (Dp), petunidin (Pt)

\footnotetext{
is Presented at the 39th International Symposium on High-Performance LiquidPhase Separations and Related Techniques, Amsterdam, Netherlands, 16-20 June 2013.

* Corresponding author. Tel.: +27 21808 3351; fax: +27 218083360.

E-mail address: ajdevill@sun.ac.za (A.de Villiers).
}

and malvidin (Mv) (Fig. 1), although more than 30 anthocyanidins have been identified [6]. Further structural variation stems from different degrees of glycosylation and acylation involving a variety of acids [7], resulting in a diverse range of anthocyanin structures: more than 560 natural anthocyanins have been identified to date $[6,8]$.

Due to this complexity, the accurate qualitative and quantitative analysis of anthocyanins remains challenging. The most widely used separation method for the analysis of anthocyanins is reversed phase liquid chromatography (RP-LC), because of its high sensitivity and selectivity, good chromatographic performance and relatively short analysis times. This mode of separation is often coupled to photodiode-array (PDA) detection, which provides some information on the nature of the aglycone as well as glycosylation and acylation patterns [9-11]. Since anthocyanins absorb in the visible range around $500-550 \mathrm{~nm}$, they can selectively be detected at these wavelengths, and therefore distinguished from other flavonoid classes [12,13]. However, LC-PDA is does not provide sufficient structural information to allow identification of individual anthocyanins, which, in light of the limited commercial availability of the reference compounds, is a major limitation. RP-LC hyphenated with mass spectrometry (MS) may provide information on both the molecular mass and molecular structure, which greatly facilitates in the identification of anthocyanins. Tandem mass spectrometry (MS/MS) is especially helpful in structural elucidation since selective fragmentation provides additional information [14,15]. Despite these benefits, however, 
(c)

Dp: $\mathrm{R}_{1}=\mathrm{R}_{2}=\mathrm{OH}, \mathrm{R}_{3}=\mathrm{R}_{4}=\mathrm{H}$

Cy: $R_{1}=O H, R_{2}=R_{3}=R_{4}=H$

Pel: $R_{1}=R_{2}=R_{3}=R_{4}=H$

Pt: $\quad \mathrm{R}_{1}=\mathrm{OCH}_{3}, \mathrm{R}_{2}=\mathrm{OH}, \mathrm{R}_{3}=\mathrm{R}_{4}=\mathrm{H}$

Pe: $R_{1}=O_{3} H_{3}, R_{2}=R_{3}=R_{4}=H$

Mv: $\mathrm{R}_{1}=\mathrm{OCH}_{3}, \mathrm{R}_{2}=\mathrm{OCH}_{3}, \mathrm{R}_{3}=\mathrm{R}_{4}=\mathrm{H}$

$\mathrm{R}_{3}, \mathrm{R}_{4}=\mathrm{H}$ or

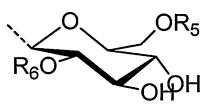

or $\mathrm{R}_{3}=$ arabinoside

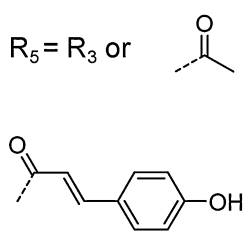

acetyl

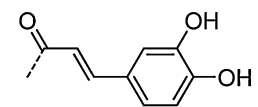

caffeoyl

p-coumaroyl

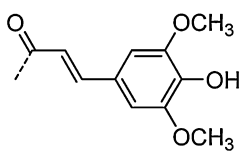

sinapoyl

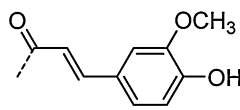

feruloyl

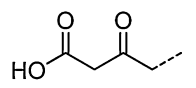

malonoyl

$\mathrm{R}_{6}=\mathrm{R}_{3}$ or $\mathrm{H}$

Fig. 1. Structures of anthocyanins identified in black beans, blueberries, red grape skins, red cabbage and red radish. Abbreviations: Dp: delphinidin, Cy: cyanidin, Pel: pelaragonidin, Pt: petunidin, Pe: peonidin, Mv: malvidin.

LC-MS alone also demonstrates some limitations for anthocyanin analysis. In positive ionisation mode (almost exclusively used for anthocyanin analyses), non-anthocyanin flavonoids such as glycosylated flavonols show identical molecular ions and fragments [16]. Moreover, MS is not capable of distinguishing between glycosidic isomers such as -glucoside and -galactosides, while the relatively simple fragmentation pattern of glycosylated anthocyanins makes the identification of related compounds in complex mixtures challenging. For these reasons, chromatographic separation of anthocyanins remains essential.

Conventional RP-LC methods display some shortcomings associated with the unique chromatographic behaviour of anthocyanins, primarily in terms of relatively low chromatographic efficiency and low optimal mobile phase flow rates, the latter implying that long analysis times are required for maximum resolution at conventional temperatures $[17,18]$. In combination with the large structural diversity of anthocyanins, this means that complete chromatographic separation of complex anthocyanin mixtures, such as encountered in for example grapes, blueberries and radish, is not always possible. Therefore the identification of anthocyanins, mostly based on the RP-LC elution order, UV-vis spectra as well as mass spectral information, is often tentative.

In order to improve the certainty in compound identification, alternative separation modes for anthocyanin analysis, such as reported for example by Vergara et al. [19] using a mixed mode ion-exchange-RP stationary phase, would be useful in terms of providing complementary selectivity. Hydrophilic interaction chromatography (HILIC, a term coined by Alpert [20]) is an aqueous variant of normal phase liquid chromatography (NP-LC) [21] which has in recent years found increasing application in phenolic analysis [22-24]. Potential advantages of HILIC include its suitability for the analysis of polar compounds that are not sufficiently retained in RP-LC, excellent compatibility with UV and especially MS detection and an alternative separation mechanism [20], which implies that compounds not easily separated by RP-LC may be resolved in this mode. As a consequence, HILIC also shows promise for hyphenation to other modes of HPLC in 2-dimensional liquid chromatography, as has been demonstrated by the HILIC $\times$ RP-LC analysis of an array of phenolic compounds [25-30].
To the best of our knowledge, HILIC has not yet been applied to the analysis of anthocyanins. The objective of this study was therefore to explore the applicability of HILIC for anthocyanin analysis. The potential of the approach will be demonstrated by the analysis of diverse anthocyanins in blueberries, grape skins, black beans, red cabbage and red radish.

\section{Experimental}

\subsection{Reagents and materials}

HPLC grade methanol, acetonitrile and trifluoroacetic acid (TFA) were purchased from Sigma-Aldrich (Steinheim, Germany) and formic acid from Merck (Darmstadt, Germany). Malvidin-3$O$-glucoside chloride was obtained from Extrasynthese (Genay, France), and dissolved at $1 \mathrm{mg} / \mathrm{mL}$ in methanol as stock solution. Deionised water was obtained using a Milli-Q water purification system (Millipore, Milford, MA, USA). All mobile phases were filtered through $0.45 \mu \mathrm{m}$ HVLP membrane filters (Millipore) and degassed in an ultrasonic bath before use. Blueberries, red cabbage, red radish, grapes and black beans were purchased from a local supermarket. The OASIS HLB solid phase extraction (SPE) cartridges (6 mL, $500 \mathrm{mg}$ ) were from Waters (Milford, MA, USA).

\subsection{Sample preparation}

Each of the five samples, i.e., blueberries, red cabbage, red radish, grape skins and black beans, were prepared in the same manner. $15 \mathrm{~g}$ of each of the samples was frozen using liquid nitrogen and ground in a coffee grinder. Anthocyanins were extracted from the ground samples with $3 \times 150 \mathrm{~mL}$ methanol/formic acid/water $(50 / 5 / 45(v / v / v))$. After filtration, the extracts were combined and concentrated to $\sim 100 \mathrm{~mL}$ on a Büchi rotary evaporator at $30^{\circ} \mathrm{C}$. Of this extract, $15 \mathrm{~mL}$ was loaded on a preconditioned ( $3 \mathrm{~mL}$ methanol and $3 \mathrm{~mL}$ water acidified with $0.1 \%$ formic acid) SPE cartridge. The cartridge was rinsed with $5 \times 1 \mathrm{~mL}$ acidified water and eluted with $2 \times 0.5 \mathrm{~mL}$ methanol/formic acid $(95 / 5(v / v))$. The methanolic fraction containing the anthocyanins was filtered through a PDVF syringe filter (Millipore) prior to HPLC analysis to remove potential particulate matter (no loss of anthocyanins was observed following this step).

\subsection{Chromatographic conditions}

\subsubsection{HPLC-UV scouting gradient conditions}

HILIC-UV analyses were performed on an Acquity UPLC system equipped with a binary bump, sample manager, column oven compartment, photodiode array (PDA) detector ( $500 \mathrm{~nL}$ flow cell, $10 \mathrm{~mm}$ path length) and controlled by Empower software (Waters). UV chromatograms were recorded at $500 \mathrm{~nm}$ using an acquisition rate of $10 \mathrm{~Hz}$. Raw UV data was exported into Origin 8.5 (OriginLab Corporation, USA) to create the figures.

Five different columns were evaluated during the initial experiments to find the stationary phase that provided the best separation. These included Develosil Diol (Nomura Chemicals, $250 \mathrm{~mm} \times 4.6 \mathrm{~mm}$ i.d., $5 \mu \mathrm{m}$ particles), Spherisorb $\mathrm{NH}_{2}$ (Waters, $250 \mathrm{~mm} \times 4.6 \mathrm{~mm}$ i.d., $5 \mu \mathrm{m}$ particles), Luna CN (Phenomenex, $250 \mathrm{~mm} \times 4.6 \mathrm{~mm}$ i.d., $5 \mu \mathrm{m}$ particles), Betasil Diol-100 (Thermo Scientific, $250 \mathrm{~mm} \times 4.6 \mathrm{~mm}$ i.d., $5 \mu \mathrm{m}$ particles), XBridge $\mathrm{BEH}$ Amide (Waters, $150 \mathrm{~mm} \times 4.6 \mathrm{~mm}$ i.d., $2.5 \mu \mathrm{m}$ particles), Kinetex HILIC (Phenomonex, $150 \mathrm{~mm} \times 2.1 \mathrm{~mm}$ i.d., $2.6 \mu \mathrm{m}$ particles) and Shodex Asahipak $\mathrm{NH}_{2} \mathrm{P}$ (Showa Denko, $250 \mathrm{~mm} \times 4.6 \mathrm{~mm}$ i.d., $5 \mu \mathrm{m}$ particles) columns. The mobile phase composition for these experiments consisted of (A) 5\% formic acid in acetonitrile and (B) 5\% formic acid in water. All experiments were performed at a flow rate of $1 \mathrm{~mL} / \mathrm{min}$ (with the exception of the Kinetex column, where the 
flow rate was $0.2 \mathrm{~mL} / \mathrm{min}$ ), ambient temperature with an injection volume of $2 \mu \mathrm{L}$. The gradient was as follows: $4-40 \% \mathrm{~B}(0-43 \mathrm{~min})$, $40 \% \mathrm{~B}$ isocratic (43-48 $\mathrm{min}$ ), 4-100\% $\mathrm{B}$ (48-53 min) before returning to initial conditions at $60 \mathrm{~min}$. For the amide column the gradient was adjusted for a $150 \mathrm{~mm}$ length column.

Apart for the experiments listed above to evaluate different stationary phases, all separations were performed on an Acquity UPLC BEH Amide column $(150 \mathrm{~mm} \times 1.0 \mathrm{~mm}$ i.d., $1.7 \mu \mathrm{m}$ particles $)$ with a UPLC pre-column filter (Waters). Three different mobile phase compositions were used during initial scouting experiments on this phase, consisting respectively of (A) 5\% formic acid/or $10 \%$ formic acid/or $0.4 \%$ trifluoroacetic acid (TFA) in acetonitrile and (B) $5 \%$ formic acid/or $10 \%$ formic acid/or $0.4 \%$ TFA in water. The flow rate was $0.05 \mathrm{~mL} / \mathrm{min}$ for all these experiments. The scouting gradient profile used was as follows: $4-40 \% \mathrm{~B}(0-24 \mathrm{~min}), 40 \%$ isocratic (24-27 $\mathrm{min}$ ), 40-100\% B (27-30 min) before returning to initial conditions at $33 \mathrm{~min}$. The column was re-equilibrated for $15 \mathrm{~min}$ before the next analysis. Analyses were performed at $25^{\circ} \mathrm{C}$ as well as $50^{\circ} \mathrm{C}$.

\subsubsection{Optimised gradient conditions for HPLC-UV and HPLC-ESI-MS analyses}

The optimised methods employed a binary mobile phase consisting of (A) $0.4 \%$ TFA in acetonitrile and (B) $0.4 \%$ TFA in water. The flow rate was $0.025 \mathrm{~mL} / \mathrm{min}$ and all analyses were performed at $50{ }^{\circ} \mathrm{C}$. Four different gradients were used; for blueberries and red grape skins: $4-35 \% \mathrm{~B}(0-35 \mathrm{~min}), 35-100 \% \mathrm{~B}$ (35-36 min), $100 \%$ B for $5 \mathrm{~min}$; red cabbage: $13-40 \%$ B (0-40 min), $40-100 \%$ B (40-41 min), $100 \%$ B for $5 \mathrm{~min}$; black beans: $5-30 \%$ B (0-27 min), 30-100\% B (27-28 min), 100\% B for 5 min; red radish: $15-33.5 \%$ B (0-36 min), $33.5-100 \%$ B (36-37 min), 100\% B for $5 \mathrm{~min}$. The column was re-equilibrated for $15 \mathrm{~min}$ before each analysis. Injection of $0.5 \mu \mathrm{L}$ was performed in a partial loop with needle overfill mode using acetonitrile/TFA $(99.6 / 0.4, v / v)$ as the weak needle wash solvent.

LC-MS, LC-MSE and LC-MS/MS analyses were performed on a Waters Acquity UPLC system equipped with a binary solvent manager, sample manager and column oven, interfaced through an electrospray ionisation (ESI) source to a Waters Synapt G2 quadrupole time-of-flight (Q-TOF) mass spectrometer. The mass spectrometer was operated in positive ionisation mode, with a capillary voltage of $2.5 \mathrm{kV}$ and a cone voltage of $35 \mathrm{~V}$. The source and desolvation temperatures were $120^{\circ} \mathrm{C}$ and $275^{\circ} \mathrm{C}$, respectively. The desolvation gas flow was $650 \mathrm{~L} / \mathrm{h}$ and the cone gas flow $50 \mathrm{~L} / \mathrm{h}$ (both $\mathrm{N}_{2}$ ). LC-MS, LC-MSE and LC-MS/MS conditions were identical except that for LC-MS ${ }^{\mathrm{E}}$ acquisition a collision energy ramp of 20-60 V was used, whereas for LC-MS/MS a static collision energy of $30 \mathrm{~V}$ was used. Data was acquired from 200 to $2000 \mathrm{amu}$ and collected and processed using MassLynx v.4.1 software (Waters). The instrument was calibrated using sodium formate solution and leucine enkephalin was used as lock mass for accurate mass determinations.

\subsubsection{Construction of van Deemter curves}

Plate height data were acquired on an XBridge BEH Amide column $(50 \mathrm{~mm} \times 4.6 \mathrm{~mm}, 2.5 \mu \mathrm{m}$ particles $)$ with a KrudKatcher pre-column filter (Phenomenex, Torrance, CA, USA) at $50^{\circ} \mathrm{C}$ on the HPLC-UV instrument. The mobile phase consisted of (A) $0.4 \%$ TFA in acetonitrile and (B) $0.4 \%$ TFA in water in the ratio $97 / 3$ (phase A/phase B). The sample contained $10 \mathrm{mg} / \mathrm{L}$ naphthalene as void-time marker and $50 \mathrm{mg} / \mathrm{L}$ malvidin-3-O-glucoside dissolved in the mobile phase. The flow rates were increased from 0.05 to $2.0 \mathrm{~mL} / \mathrm{min}$. 10 different flow rates were used and all measurements were performed in duplicate. UV detection was performed at 280 and $500 \mathrm{~nm}$, with an acquisition rate of $10 \mathrm{~Hz}$. To correct for the system contribution to band broadening, the column was replaced with a union and naphthalene was injected under the same conditions.

\section{Results and discussion}

\subsection{HILIC method optimisation}

\subsubsection{Evaluation of stationary phases}

HILIC methods previously reported for the separation of proanthocyanidins, flavonols and chalcones by our group [25-27] were used as starting point for the development of a HILIC method for anthocyanins. Initial HILIC-UV experiments were performed using mobile phases consisting of $5 \%$ formic acid in acetonitrile (solvent $A$ ) and $5 \%$ formic acid in water (solvent B). The use of highly acidic mobile phases was dictated by the unique chromatographic behaviour of anthocyanins (see further). A range of different stationary phases was evaluated, including silica (Kinetex HILIC), diol (Develosil Diol, Betasil Diol-100), amino (Spherisorb $\mathrm{NH}_{2}$, Shodex Asahipak $\mathrm{NH}_{2} \mathrm{P}$ ), cyanopropyl (Luna $\mathrm{CN}$ ) and amide (BEH Amide) phases. With the exception of the cyanopropyl, amide and diol phases, all columns showed very poor retention of anthocyanins (Figure S1).

In the case of the bare silica column, the lack of retention is most likely due to the suppression of ion exchange interaction involving free silanols under the highly acidic mobile phase conditions dictated for anthocyanin analysis [31]. For the amino columns, protonation of stationary phase is responsible for exclusion of the positively charged flavylium cations (the same behaviour was observed for aminopropyl (Spherisorb $\mathrm{NH}_{2}$ ) and polyamine-functionalised polymeric (Shodex $\mathrm{NH}_{2} \mathrm{P}$ ) phases). While the cyanopropyl bonded phase showed some retention, the selectivity was very poor, with all anthocyanins eluting as a single peak. In contrast, the diol phases showed higher retention and partial separation of anthocyanins under these conditions. Significant differences were however observed between the two diol phases evaluated, with the Develosil phase providing improved retention and separation. These results are in accordance with previous reports which highlighted the suitability of this diol phase for procyanidin analysis [26,27,32] and might be associated with higher carbon load and the lack of endcapping of the Develosil phase compared to the Betasil phase. Of all the columns evaluated, the BEH Amide phase showed the best performance in terms of both retention and selectivity. The high retention of cationic species on the amide phase is in accordance with previous reports [29]. On the column used here, retention is likely associated with the amide functionality and not the ethyl-bridged (BEH) particle, as much higher retention has previously been demonstrated for basic compounds on the BEH Amide phase compared to a native $\mathrm{BEH}$ phase [33], also considering that ion exchange is expected to play a minimal role in retention under the highly acid mobile phase conditions used. It would therefore be interesting to evaluate alternative amide phases, such as for example the carbamoyl-based TSK-gel Amide, in future work to determine whether these phases might provide improved performance for this application.

Since the most promising results were obtained on the $\mathrm{BEH}$ Amide phase, the scouting gradient was adapted on this column to ensure elution of anthocyanins in all five samples across the entire gradient profile (Fig. 2). It is immediately evident that, with the exception of red radish, anthocyanins in all the other samples were characterised by very broad peaks. Red radish is unique amongst the samples investigated in that it contains pelaragonidin-tri-glycosides varying in their acylation patterns. Considering that the other acylated anthocyanins show markedly broader peaks (for example acylated cyanidin-triglycosides in red cabbage), this behaviour is presumably related to the nature of the 


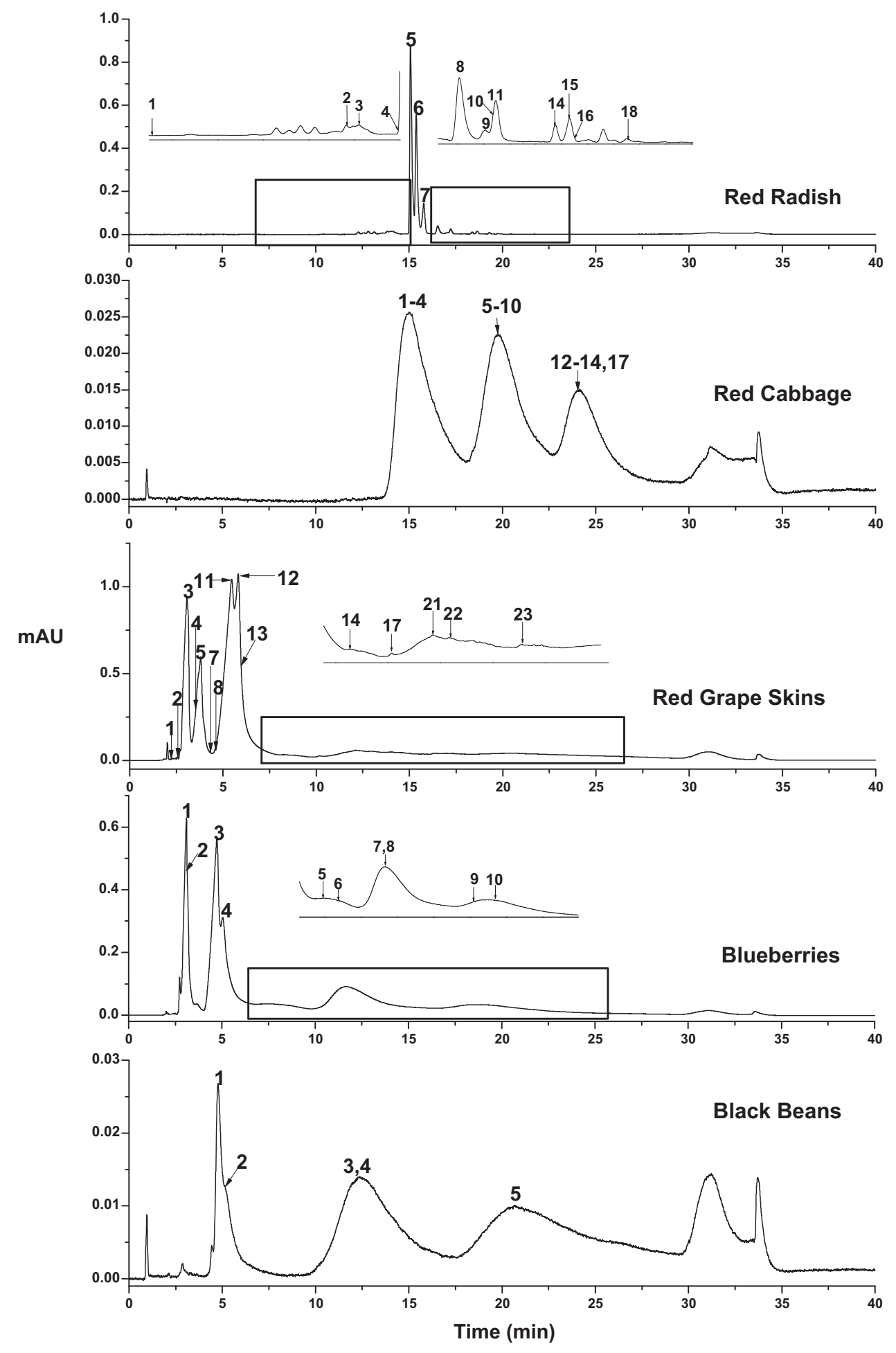

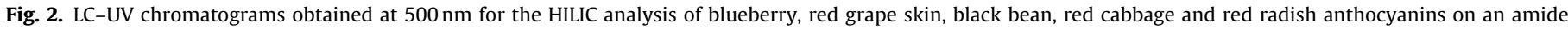

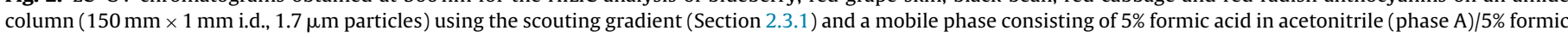

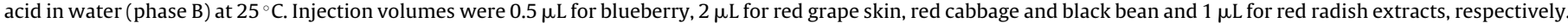
Peak numbers correspond to Table 1.

anthocyanidin moiety. The divergent chromatographic characteristics of pelaragonidin species are likely linked to differences in the kinetics of the hydration reaction between flavylium cationic and carbinol pseudobasic forms of this anthocyanidin. According to data reported by Leydet et al. [34], the equilibrium constant for the hydration reaction of pelarogonidin-3-O-glucoside is about $40-60 \%$ lower than for the other five anthocyanins (due to a faster "reverse" rate constant for this reaction in the case of pelargonidin). Due to the smaller percentage of pseudobasic species of pelargonidin derivatives present at a given $\mathrm{pH}$, the effect of the hydration reaction on chromatographic efficiency is therefore also expected to be reduced. This observation is consistent with less peak broadening evident for pelaragonidin species in red radish. 
The higher retention evident for red radish and cabbage anthocyanins compared to the other samples is due to the presence of di-, tri- and tetra-glycosylated species of cyanidin and pelarogonidin in the former two samples (see further).

In order to develop a generic HILIC method suitable for the analysis of diverse natural anthocyanins, further optimisation of especially the mobile phase composition was required to improve the chromatographic performance, as discussed briefly in the following sections.

\subsubsection{Optimisation of mobile phase composition}

The prevalent mobile phase $\mathrm{pH}$ has a profound effect on the solution chemistry (and colour properties) of anthocyanins [7,35], and should therefore be considered in HPLC method development. Extensive research by Brouillard and co-workers [36-38] as well as other researchers has demonstrated that anthocyanins exist in solution as mixtures of different species: the flavylium cation $\left(\mathrm{AH}^{+}\right)$, quinoidal base (A), carbinol pseudobase (B) and chalcone (C) forms (Fig. 3). The flavylium cationic species, which is responsible for visible absorption at $\sim 520 \mathrm{~nm}$, and therefore the red colour of most anthocyanins, predominates at low pH (below 3) [17]. With an increase in $\mathrm{pH}$, thermodynamic and kinetic competition between the hydration of the flavylium cation and proton transfer involving the acidic hydroxyl group occurs. Hydration of the flavylium cationic species leads to formation of a carbinol pseudo-base, which can undergo further ring opening to yield the yellow chalcone.

The chromatographic implications of these secondary reactions have been studied by de Villiers et al. [17,18]. Highly acidic mobile phases are essential to ensure prevalence of the flavylium cationic species and improved chromatographic performances, since interspecies conversions that occur at higher $\mathrm{pH}$ severely compromise efficiency. Further improvements may be obtained by operating at elevated temperature and at the optimal mobile phase linear velocity $[17,18]$. Previous work on the topic was however focused exclusively on RP-LC separation; therefore, the effect of mobile phase $\mathrm{pH}$ in HILIC was also evaluated here.

Under the chromatographic conditions used for the scouting analysis (Fig. 2), i.e. acetonitrile/water containing $5 \%$ formic acid, the $\mathrm{pH}$ at the start of the gradient was measured as 0.54 . This value was obtained using a standard $\mathrm{pH}$ electrode calibrated in aqueous reference solutions, denoted ${ }_{s}^{w} \mathrm{pH}$. While this value would be sufficiently low (in an aqueous environment) to ensure that anthocyanins are virtually exclusively present in the flavylium cationic form, it should be considered that under HILIC conditions, i.e. where a high percentage of organic modifier is used, the effective $\mathrm{pH},{ }_{s}^{s} \mathrm{pH}$, is much higher [39]. According to the conversion protocol reported by Gagliardi et al. [39], the effective $\mathrm{pH}$ of $5 \%$ formic acid in $96 \%$ acetonitrile would be $\sim 2.20$. This value is indeed in the region where a significant portion of anthocyanins will be present in the carbinol pseudobase form, and therefore where secondary equilibria are expected to affect chromatographic performance [17]. In reality the situation is probably much more complex, since the primarily aqueous environment close to the stationary phase would effectively have a lower $\mathrm{pH}$ than the bulk of the mobile phase. This is primarily due to the higher activity coefficients of protons in the aqueous environment, and may be enhanced by potential partitioning of the acidic component of the mobile phase in the aqueous layer surrounding the stationary phase. It is therefore likely that an effective $\mathrm{pH}$ gradient is formed between the layer of solvent associated with the stationary phase and the bulk mobile phase, which will affect anthocyanin equilibria. To study the effect of these phenomena is exceptionally hard. Nevertheless, the fact that the effective $\mathrm{pH}$ in the bulk mobile phase is higher than in predominantly aqueous mobile phases containing the same acid content remains valid, and this is expected to affect the chromatographic behaviour of anthocyanins.
To verify this hypothesis, and confirm that secondary equilibria are responsible for the broad peaks observed in Fig. 2, the acid content of the mobile phase was increased to $10 \%$ formic acid, keeping all other conditions identical. The ${ }_{s}^{w} \mathrm{pH}$ and ${ }_{s}^{s} \mathrm{pH}$ values for this mobile phase under initial HILIC conditions are -0.74 and $\sim 0.92$, respectively. A comparison between $5 \%$ and $10 \%$ formic acid mobile phases (refer to Figure S2) indeed shows improved peak shapes for the more acidic mobile phase, especially in the case of black bean and red cabbage anthocyanins, thereby confirming that $\mathrm{pH}$ plays an important role in the chromatographic efficiency in HILIC. In addition, retention for virtually all analytes decreased significantly with an increase in formic acid content, as expected for a more polar mobile phase.

The use of trifluoroacetic acid (TFA) as acidic mobile phase component was also investigated, as TFA is known to impart unique selectivity, especially in the case of anthocyanins, where ionpairing plays a role in their RP-LC separation [40]. The scouting gradient was therefore performed using $0.4 \%(v / v)$ TFA instead of formic acid. ${ }_{s}^{w} \mathrm{pH}$ and ${ }_{s}^{s} \mathrm{pH}$ values for this mobile phase under initial HILIC conditions were -0.11 and $\sim 1.55$, respectively, therefore slightly higher than for a $10 \%$ formic acid mobile phase. Despite this, however, anthocyanin peak shapes for all samples improved dramatically with the TFA phases. An increase in retention for all anthocyanins may be largely ascribed to the lower polarity of the mobile phase containing $0.4 \%$ TFA compared to $10 \%$ formic acid. Counteracting this effect, ion-pairing of anthocyanins with TFA is likely to occur, which would decrease retention. The effect of ionpairing is clearly overshadowed by the differences in mobile phase polarity. However, the ion-pair reaction with the flavylium cation is expected to compete with the hydration reaction, and this possibly contributes to a reduction in band broadening due to the latter equilibrium. As example, Fig. $4 a-c$ illustrates the effect of mobile phase composition and $\mathrm{pH}$ on the HILIC analysis of black bean anthocyanins. A complete overview of the analysis of each of the samples using each of the mobile phase compositions discussed above is presented in Figure S2.

\subsubsection{Effect of temperature}

Increasing the temperature has been shown to be an effective means of improving the chromatographic performance for the separation of anthocyanins [17,18]. This follows from the fact that higher temperatures result in an increase in the rate constant for the hydration reaction, thereby leading to a reduction in band broadening due to secondary equilibria [17]. The effect of increasing the analysis temperature to $50{ }^{\circ} \mathrm{C}$ was therefore evaluated using the $0.4 \%$ TFA mobile phase. It should be noted that, although anthocyanins are susceptible to thermal degradation, it has been shown that thermal degradation does not pose a risk at this temperature for analysis times less than $1 \mathrm{~h}$ [17]. Comparison of Fig. 4c and d clearly confirms that a noteworthy improvement in peak shape and resolution is obtained at the higher temperature for black bean anthocyanins. Figure S3 presents an overview of the effect of analysis temperature for all investigated samples. A similar improvement with increasing temperature was observed for the formic acid mobile phases (results not shown), as previously also shown for RP-LC separations $[17,18]$.

\subsubsection{Optimisation of mobile phase flow rate}

Previous work has shown that anthocyanins, when analysed by RP-LC, exhibit exceptionally low optimal linear velocities and elevated plate heights. This implies drastic losses in efficiency and/or speed under conventional RP-LC conditions. In general, HILIC shows much lower chromatographic efficiency, as well as lower optimal flow rates, compared to RP-LC [41]. In order to get an idea of the optimal flow rate for the HILIC analysis of anthocyanins, plate height curves were constructed using the optimal 


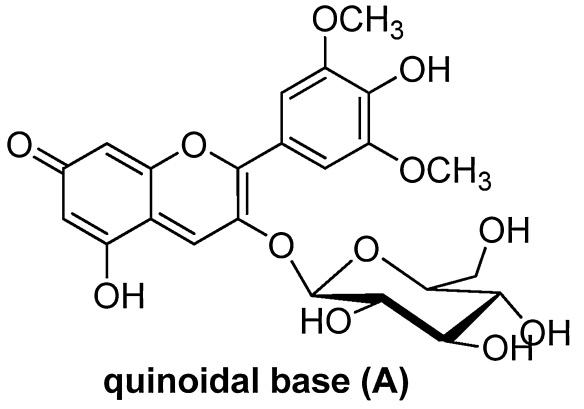<smiles>[Y20]C#CC#C</smiles>

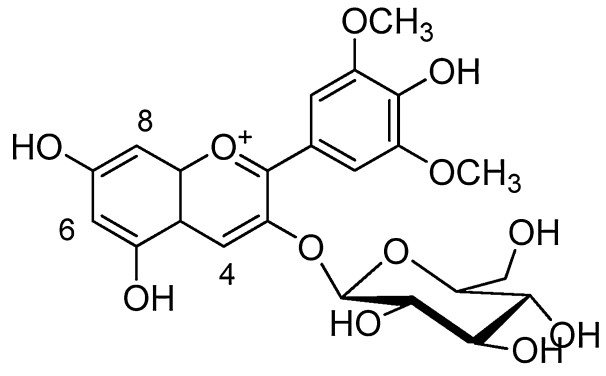

flavylium cation $\left(\mathrm{AH}^{+}\right)$<smiles>COc1cc(C(=O)/C(=C\c2c(O)cc(O)cc2O)O[C@@H]2O[C@H](CO)[C@@H](O)[C@H](O)[C@H](O)[C@H]2O)cc(OC)c1O</smiles>

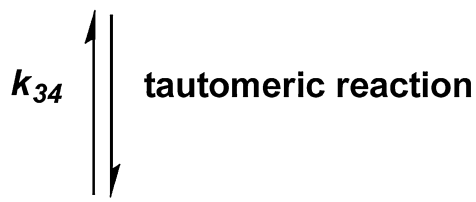<smiles>C=CC(=O)O</smiles><smiles>COC1=CC2=CC(OC)=C(O1)C(O[C@H]1C(O)C[C@@H](O)[C@H](O)[C@H]1O)OC1=Cc3c(O)cc(O)cc3OC21O</smiles>

carbinol pseudobase (B)

Fig. 3. The pH-dependant equilibria between the different chemical forms of anthocyanins, demonstrated for malvidin-3-O-glucoside.

conditions ascertained previously: a mobile phase containing $0.4 \%$ TFA in acetonitrile and water at $50^{\circ} \mathrm{C}$. Malvidin-3-O-glucoside was used as representative compound in these experiments and a $50 \mathrm{~mm} \times 4.6 \mathrm{~mm}, 2.5 \mu \mathrm{m} d_{p}$ Amide column was used to minimise the effect of extra-column band broadening.

The plate height curve obtained on this column is presented in Fig. 5. The most striking feature of these data is the exceptionally low optimal linear velocity: the value of $\sim 0.1 \mathrm{~mm} / \mathrm{s}$ corresponds to an optimal flow rate of $\sim 0.07 \mathrm{~mL} / \mathrm{min}$ on a $4.6 \mathrm{~mm}$ i.d. column ( $\sim 4 \mu \mathrm{L} / \mathrm{min}$ on a $1 \mathrm{~mm}$ i.d. column). Furthermore, the minimum plate height value of $20.15 \mu \mathrm{m}$ corresponds to about $8 d_{p}$, which is much higher than the norm of $\sim 2 d_{p}$ for RP-LC, and even compared to other HILIC separations [41]. Analogously to RP-LC [17], these results demonstrate a residual effect of secondary equilibria even at $50^{\circ} \mathrm{C}$, which might be further exacerbated by mixed-mode HILIC retention mechanisms [41].

For the $150 \mathrm{~mm} \times 1.0 \mathrm{~mm}$, i.d., $1.7 \mu \mathrm{m} d_{p}$ Amide column used for gradient analysis in this study, the optimal flow rate would be slightly higher $(\sim 6 \mu \mathrm{L} / \mathrm{min})$ due to the smaller particle size. However, due to practical considerations such as pumping accuracy and analysis times, gradient analyses were performed at $25 \mu \mathrm{L} / \mathrm{min}$ on this column. These conditions were found to provide adequate results on a low-dispersion instrument.

\subsection{Application of HILIC-ESI-MS(/MS) to the analysis of anthocyanins in natural products}

Based on the optimal HILIC experimental conditions derived for anthocyanin analysis (Section 3.1), gradient profiles were finetuned for the analysis of each of the samples under investigation. These optimised methods were coupled to high resolution ESI-QTOF MS detection to identify anthocyanins in each of the samples.
The general identification strategy based on MS data will be outlined below, followed by a more detailed discussion on the HILIC separation of anthocyanins in each of the samples investigated. Note that no quantification was performed in the current study due to a lack of authentic standards. However, the sensitivity of the developed methods was sufficient to allow detection of trace-level $(<1 \mathrm{mg} / \mathrm{kg}$ ) [42] anthocyanins in for example grape skin extracts.

\subsubsection{HILIC-ESI-MS(/MS) identification of anthocyanins}

ESI-MS detection was performed in positive ionisation mode, since anthocyanins are predominantly present as flavylium cations under the acidic mobile phase conditions, facilitating the production of molecular cations $[\mathrm{M}]^{+}$in the highest yields. Compounds were tentatively identified based on accurate mass measurements in combination with molecular formulae as well as by comparison of UV-, and MS spectra and primary fragmentation patterns compared to literature reports [13,43-46]. Retention characteristics relative to RP-LC were also taken into account (refer to Section 3.3 further).

The Q-TOF instrument was programmed to acquire data in scan mode for molecular ion information, while at the same time acquiring $\mathrm{MS}^{\mathrm{E}}$ data, where a ramp of collision cell energy is used to obtain fragmentation information for any ions present in the collision cell. Targeted MS/MS experiments were also performed for selected coeluting compounds in black beans and red cabbage. Examples of typical MS ${ }^{\mathrm{E}}$ and MS/MS mass spectra for different classes of anthocyanins are presented in Fig. 6a-f. It should be noted that while mobile phases containing relatively high quantities of TFA such as used here are often considered incompatible with MS detection due to potential ion suppression and source contamination, acceptable results were obtained using the developed HILIC methods. Some ion suppression compared to mobile phases containing formic acid was 


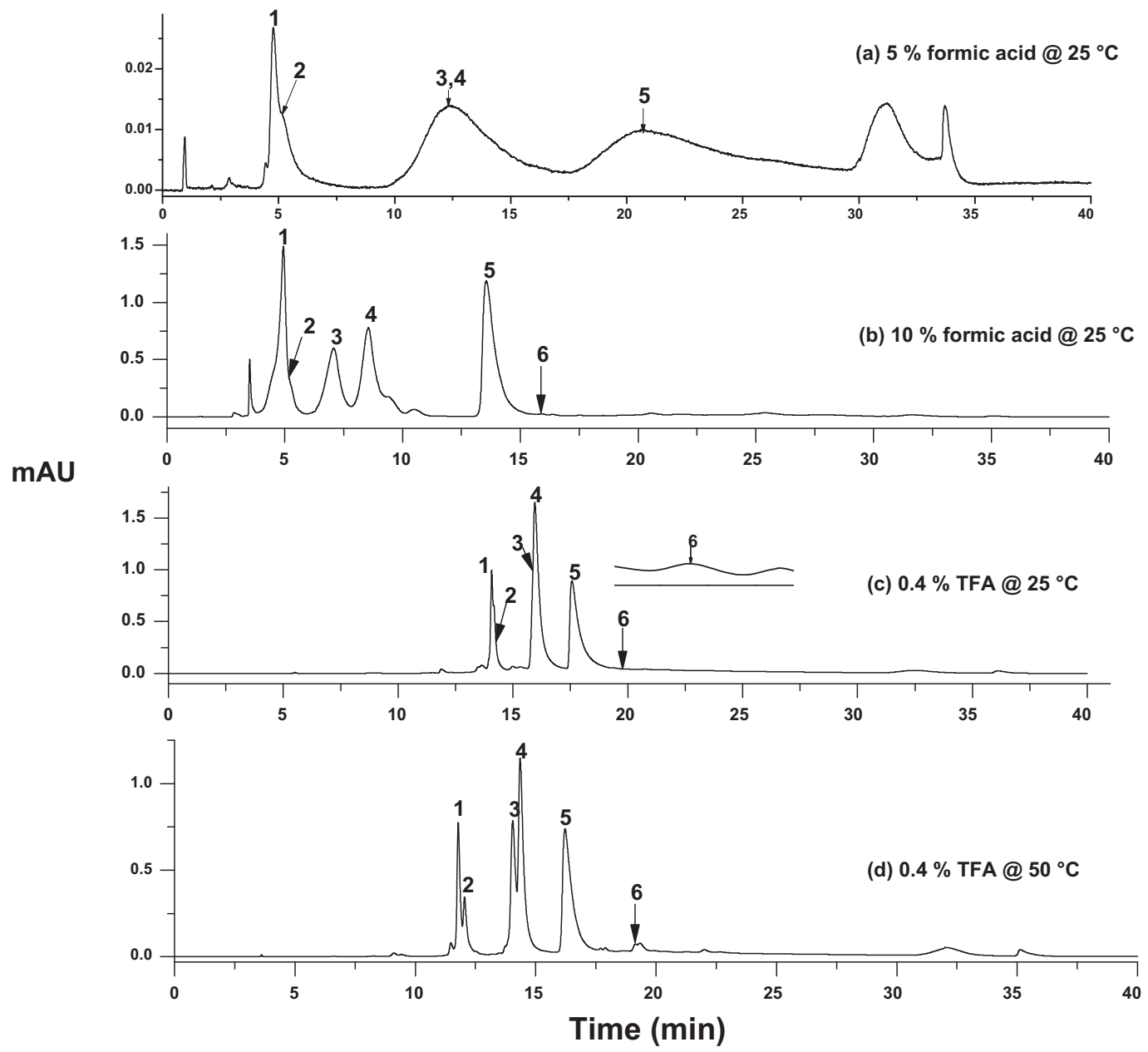

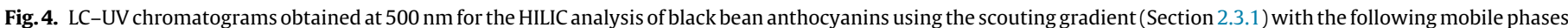

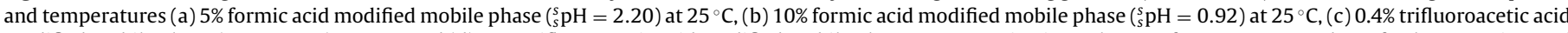

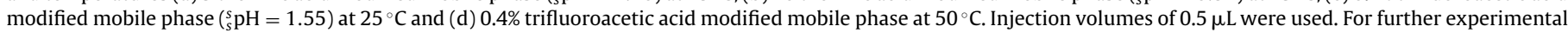
detail, refer to Section 2.3.1. Peak numbers correspond to Table 1.

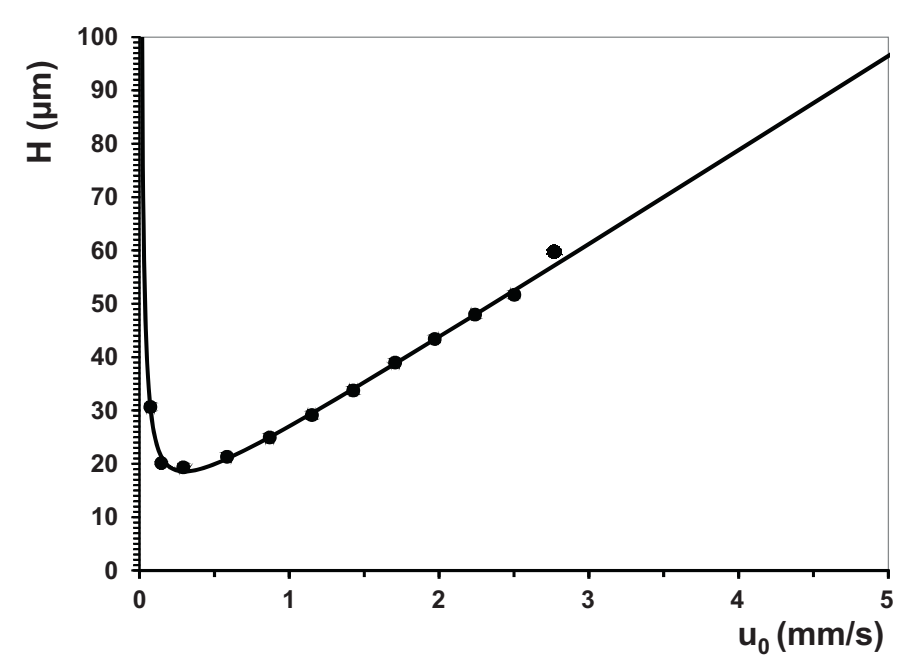

Fig. 5. Experimental van Deemter curve obtained for malvidin-3-O-glucoside on an XBridge BEH Amide column $\left(50 \mathrm{~mm} \times 4.6 \mathrm{~mm}\right.$ i.d., $\left.2.5 \mu \mathrm{m} d_{p}\right)$. Mobile phase composition: $97 \%$ phase $\mathrm{A} / 3 \%$ phase $\mathrm{B}$ (acetonitrile/water, both containing $0.4 \% \mathrm{TFA}$ ), column temperature $50^{\circ} \mathrm{C}$. observed, yet sufficient sensitivity was obtained to allow accurate detection of even trace-level anthocyanins using MS and MS/MS. In practice cleaning of the sample cone was required as well as thorough rinsing to clean the system from TFA for subsequent analyses.

The common glycosylation sites for anthocyanins are the 3-and 5-hydroxyl positions on the $C$ and A rings, respectively (Fig. 1). In addition, various acylated derivatives, as well as several diand triglucosides of the 6 main anthocyanindin bases (delphinidin, cyanidin, petunidin, peonidin, pelargonidin and malvidin) were detected. The anthocyanidin bases were detected as base peaks in the $\mathrm{MS}^{\mathrm{E}}$ mass spectra, following the loss of for example $\mathrm{m} / \mathrm{z}$ 162 or 132 (monoglucosides), $m / z 204$ (acetyl-glucosides), $m / z$ 308 (coumaroyl-glucoside) and $\mathrm{m} / \mathrm{z} 324$ (diglucosides or caffeoylglucosides), etc. $[12,14]$. As is common for acylated anthocyanins, fragmentation therefore involves cleavage of the intact acylglycosidic group [15].

Furthermore, under $\mathrm{MS}^{\mathrm{E}}$ conditions, some fragmentation of the anthocyanidin base was also observed. Significantly, under high collision energy conditions, this fragmentation pattern is characteristic and allows the identification of the relevant anthocyanidin $[12,14]$. For example, in the case of methoxylated anthocyanidins such as peonidin (Fig. 6a-c), losses of $\mathrm{CH}_{3}{ }^{\bullet}, \mathrm{CHO}{ }^{\bullet}, \mathrm{CO}$ and/or $\mathrm{CH}_{3} \mathrm{OH}$ are dominant, and this fragmentation pattern is largely independent of the glycosylation/acylation substitution of the base $[14,47]$. 

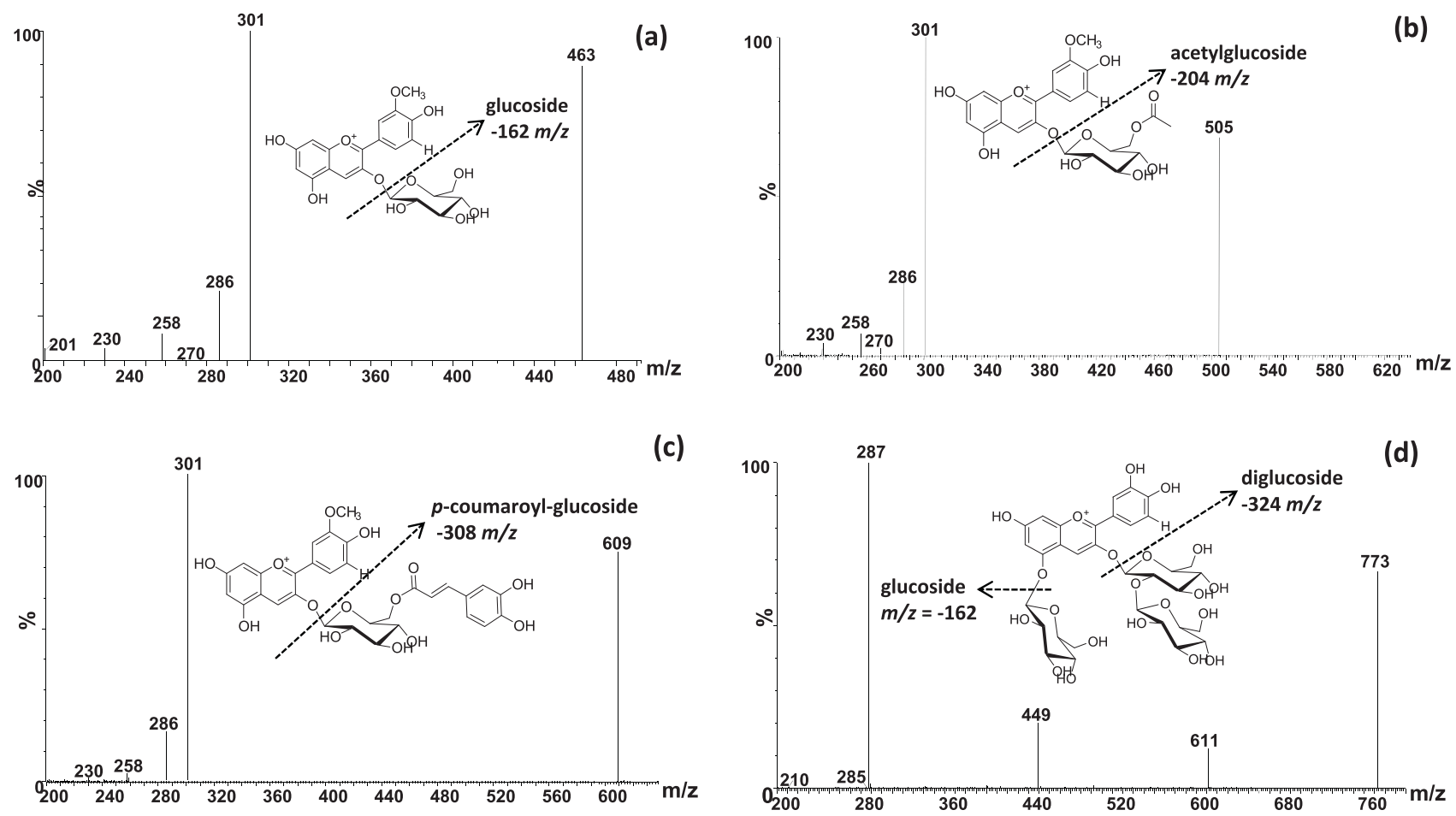

(f)
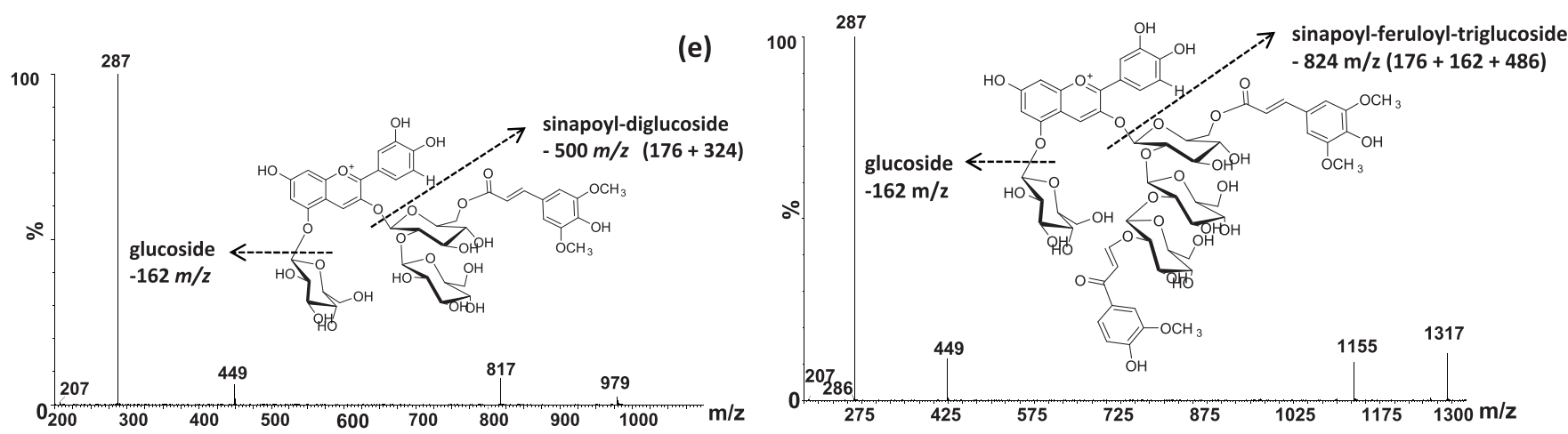

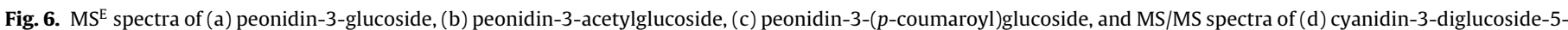

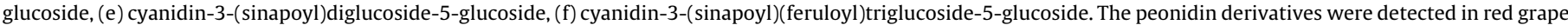
skins and the cyanidin derivatives in red cabbage.

$\mathrm{MS}^{\mathrm{E}}$ as well as MS/MS spectra are also very informative for the anthocyanidin-di-O-glycosides and their acylated derivatives such as found in red cabbage and red radish. For example, the MS ${ }^{\mathrm{E}}$ spectrum obtained for cyanidin-3-glucoside-5-diglucoside (Fig. 6d) shows three major fragment ions at $m / z 449,611$ and 287, respectively. The ions at $m / z 611$ and $m / z 449$ correspond to the loss of glucoside $(-162 \mathrm{~m} / \mathrm{z})$ and di-glucoside $(-324 \mathrm{~m} / \mathrm{z})$ groups, respectively, while the ion at $m / z 287$ identifies the aglycone (cyanidin). For these molecules, cleavage of the inter-glycosidic bond is not common (with the exception of anthocyanidin-rutinosides [15]), and therefore di-hexosides (or caffeoyl-glucosides) are readily identified by the loss of $m / z$ 324. For acylated anthocyanidin-di$O$-glucosides, similar fragmentation behaviour was observed, with the acyl-(di)glycoside moiety being cleaved intact during fragmentation. For example, cyanidin-3-(sinapoyl)diglucoside-5-glucoside (6) displayed fragment ions at $m / z 817,449$ and 287 (Fig. 6e). These correspond to the loss of glucose (162), sinapoyl-glucose $(162+206)$ and loss of both groups to result in the aglycone cyanidin ion. Fig. $6 \mathrm{f}$ shows the fragmentation patterns of highly acylated derivative of cyanidin-3-diglucoside-5-glucoside. Fragment ions at $\mathrm{m} / \mathrm{z} 1155,449$ and 287 correspond to loss of 162 (glucose), 706 $(324+382)$ and 868 (i.e. $706+162) \mathrm{m} / z$. The loss of 382 indicates the presence of more than one acylated group, and this compounds was therefore identified as cyanidin-3-(sinapoyl)(feruloyl)triglucoside5 -glucoside (8), previously identified in red cabbage $[46,48]$.

The remainder of the anthocyanins was tentatively identified using the same criteria as outlined above, details being provided in Table 1. Note that, in the absence of available standards, the identities of the specific hexoside or pentosides could not be established based on MS, MS ${ }^{\mathrm{E}}$ and MS/MS data alone. In addition, distinction between -glucoside and -galactoside isomers was not possible, since these compounds co-eluted in HILIC (see further). The same applies to the position and sequence of glycosylation (i.e. 3- or 5glycosides, especially in the case of di- and tri-glycosides) and to the acylation position (acylation at $3^{\prime}-$ and $6^{\prime}$ - positions are most commonly encountered). For unambiguous structural elucidation, complementary techniques such as nuclear magnetic resonance (NMR) of LC-NMR would be required [43]. Tentative compound 
Table 1

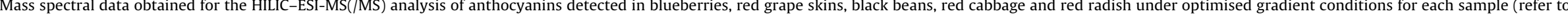
Section 2.3.2 for details).

\begin{tabular}{|c|c|c|c|c|c|c|c|c|}
\hline $\begin{array}{l}\text { Compound } \\
\text { number }\end{array}$ & $t_{R}(\min )$ & Identity & $\begin{array}{l}\text { Molecular } \\
\text { formula }[\mathrm{M}]^{+}\end{array}$ & $\begin{array}{l}\text { Experimental } \\
\text { mass }(m / z)\end{array}$ & Error $(\mathrm{ppm})$ & $\mathrm{MS}^{\mathrm{E}}$ fragments & MS/MS fragments & References \\
\hline \multicolumn{9}{|l|}{ Black beans } \\
\hline 1 & 18.16 & Malvidin-3-glucoside & $\mathrm{C}_{23} \mathrm{H}_{25} \mathrm{O}_{12}$ & 493.1343 & -0.6 & $\begin{array}{l}331.08^{* \mathrm{~b}}, 315.05,287.06, \\
242.06\end{array}$ & $\begin{array}{l}331.08^{* \mathrm{~b}}, 315.05,287.06,242.06 \\
213.06\end{array}$ & [45] \\
\hline 2 & 18.76 & Peonidin-3-glucoside & $\mathrm{C}_{22} \mathrm{H}_{23} \mathrm{O}_{11}$ & 463.1235 & -1.1 & $301.07^{* \mathrm{~b}}$ & $301.07^{* \mathrm{~b}}, 286.05,258.05$ & [45] \\
\hline 3 & 22.03 & Petunidin-3-glucoside & $\mathrm{C}_{22} \mathrm{H}_{23} \mathrm{O}_{12}$ & 479.1189 & -0.2 & $317.07^{* \mathrm{~b}}, 302.04$ & $\begin{array}{l}317.07^{* \mathrm{~b}}, 302.04,274.05,245.05 \\
217.05,203.04\end{array}$ & [45] \\
\hline 4 & 22.47 & Cyanidin-3-glucoside & $\mathrm{C}_{21} \mathrm{H}_{21} \mathrm{O}_{11}$ & 449.1085 & 0.2 & $287.06^{* \mathrm{~b}}, 213.05$ & $287.06^{* \mathrm{~b}}, 213.06$ & [45] \\
\hline 5 & 25.38 & Delphinidin-3-glucoside & $\mathrm{C}_{21} \mathrm{H}_{21} \mathrm{O}_{12}$ & 465.1035 & 0.4 & $303.05^{* \mathrm{~b}}$ & n.p.c & [45] \\
\hline 6 & 27.13 & Delphinidin-3,5-diglucoside & $\mathrm{C}_{27} \mathrm{H}_{31} \mathrm{O}_{17}$ & 627.1550 & -1.8 & $465.10,303.05^{* b}$ & n.p. ${ }^{c}$ & [45] \\
\hline \multicolumn{9}{|l|}{ Blueberries } \\
\hline 1 & 12.42 & Malvidin-3-arabinoside & $\mathrm{C}_{22} \mathrm{H}_{23} \mathrm{O}_{11}$ & 463.1240 & 0.0 & $\begin{array}{l}331.08^{* b}, 315.05,287.06, \\
242.06\end{array}$ & n.p. ${ }^{c}$ & {$[45,46]$} \\
\hline 2 & 13.32 & Peonidin-3-arabinoside & $\mathrm{C}_{21} \mathrm{H}_{21} \mathrm{O}_{10}$ & 465.1140 & 1.2 & $301.07^{* \mathrm{~b}}$ & n.p. ${ }^{c}$ & {$[45,46]$} \\
\hline 3 & 18.59 & Malvidin-3-glucoside & $\mathrm{C}_{23} \mathrm{H}_{25} \mathrm{O}_{12}$ & 493.1346 & 0.0 & $331.08^{* \mathrm{~b}}, 315.05,242.06$ & n.p. ${ }^{c}$ & {$[45-47]$} \\
\hline 4 & 19.12 & Peonidin-3-glucoside & $\mathrm{C}_{22} \mathrm{H}_{23} \mathrm{O}_{11}$ & 463.1240 & 0.0 & $\begin{array}{l}301.07^{* \mathrm{~b}}, 286.05,258.05, \\
149.02\end{array}$ & n.p. ${ }^{c}$ & {$[45-47]$} \\
\hline 5 & 19.50 & Petunidin-3-arabinoside & $\mathrm{C}_{21} \mathrm{H}_{21} \mathrm{O}_{11}$ & 449.1091 & 1.6 & $317.07^{* \mathrm{~b}}, 287.06$ & n.p. ${ }^{c}$ & [45-47] \\
\hline 6 & 20.24 & Cyanidin-3-arabinoside & $\mathrm{C}_{20} \mathrm{H}_{19} \mathrm{O}_{10}$ & 419.0979 & 0.2 & $287.06^{* \mathrm{~b}}, 213.06,149.03$ & n.p. ${ }^{c}$ & {$[45,46]$} \\
\hline 7 & 23.28 & Petunidin-3-glucoside & $\mathrm{C}_{22} \mathrm{H}_{23} \mathrm{O}_{12}$ & 479.1197 & 1.5 & $\begin{array}{l}317.07^{* b}, 302.04,274.05, \\
149.02\end{array}$ & n.p. ${ }^{c}$ & [45-47] \\
\hline 8 & 23.49 & Cyanidin-3-glucoside & $\mathrm{C}_{21} \mathrm{H}_{21} \mathrm{O}_{11}$ & 449.1080 & -0.9 & $287.06^{* \mathrm{~b}}, 213.05,149.03$ & n.p. ${ }^{c}$ & [45-47] \\
\hline 9 & 23.95 & Delphinidin-3-arabinoside & $\mathrm{C}_{20} \mathrm{H}_{19} \mathrm{O}_{11}$ & 435.0925 & -0.5 & $303.05^{* b}$ & n.p. ${ }^{c}$ & {$[45,46]$} \\
\hline 10 & 26.75 & Delphinidin-3-glucoside & $\mathrm{C}_{21} \mathrm{H}_{21} \mathrm{O}_{12}$ & 465.1040 & 1.5 & $303.05^{* \mathrm{~b}}, 149.03$ & n.p. ${ }^{c}$ & [45-47] \\
\hline \multicolumn{9}{|c|}{ Red grape skins } \\
\hline 1 & 7.07 & $\begin{array}{l}\text { Acetaldehyde derivative of } \\
\text { malvidin-3-actylglucoside (Acetylvitisin B) }\end{array}$ & $\mathrm{C}_{27} \mathrm{H}_{27} \mathrm{O}_{13}$ & 559.1452 & 5.5 & $355.07^{* \mathrm{~b}}$ & n.p. ${ }^{c}$ & {$[13,53-56]$} \\
\hline 2 & 9.12 & $\begin{array}{l}\text { Acetaldehyde derivative of } \\
\text { malvidin-3-( } p \text {-coumaroyl) glucoside } \\
\text { (Coumaroylvitisin B) }\end{array}$ & $\mathrm{C}_{34} \mathrm{H}_{31} \mathrm{O}_{14}$ & 663.1714 & 3.8 & $355.08^{* \mathrm{~b}}$ & n.p. ${ }^{c}$ & {$[13,53-56]$} \\
\hline 3 & 10.69 & $\begin{array}{l}\text { Acetaldehyde derivative of } \\
\text { malvidin-3-glucoside (Vitisin B) }\end{array}$ & $\mathrm{C}_{25} \mathrm{H}_{25} \mathrm{O}_{12}$ & 517.1373 & 5.2 & $355.09^{* \mathrm{~b}}$ & n.p. ${ }^{c}$ & {$[12-14,53-56]$} \\
\hline 4 & 12.74 & Malvidin-3-acetylglucoside & $\mathrm{C}_{25} \mathrm{H}_{27} \mathrm{O}_{13}$ & 535.1453 & 0.2 & $331.08^{* \mathrm{~b}}, 315.05,242.06$ & n.p. ${ }^{c}$ & {$[41,45]$} \\
\hline 5 & 13.38 & Peonidin-3-acetylglucoside & $\mathrm{C}_{24} \mathrm{H}_{25} \mathrm{O}_{12}$ & 505.1346 & 0.0 & $301.07^{* b}, 286.05$ & n.p. ${ }^{c}$ & {$[45,46]$} \\
\hline 6 & 15.24 & $\begin{array}{l}\text { Pyruvic acid derivative of } \\
\text { malvidin-3-acetylglucoside (Acetylvitisin A) }\end{array}$ & $\mathrm{C}_{28} \mathrm{H}_{27} \mathrm{O}_{15}$ & 603.1350 & 0.5 & $399.07^{* \mathrm{~b}}$ & n.p. ${ }^{c}$ & {$[13,51-54]$} \\
\hline 7 & 17.00 & Malvidin-3-( $p$-coumaroyl) glucoside & $\mathrm{C}_{32} \mathrm{H}_{31} \mathrm{O}_{14}$ & 639.1717 & 0.5 & $331.08^{* \mathrm{~b}}$ & n.p. ${ }^{c}$ & {$[42,45]$} \\
\hline 8 & 17.38 & Peonidin-3-( $p$-coumaroyl) glucoside & $\mathrm{C}_{31} \mathrm{H}_{29} \mathrm{O}_{13}$ & 609.1613 & 0.8 & $\begin{array}{l}301.07^{* \mathrm{~b}}, 286.05,258.05, \\
147.02\end{array}$ & n.p. ${ }^{c}$ & {$[42,45]$} \\
\hline 9 & 18.19 & $\begin{array}{l}\text { Pyruvic acid derivative of } \\
\text { malvidin-3-( } p \text {-coumaroyl) glucoside } \\
\text { (Coumaroylvitisin } A \text { ) }\end{array}$ & $\mathrm{C}_{35} \mathrm{H}_{31} \mathrm{O}_{16}$ & 707.1609 & -0.4 & $399.07^{* \mathrm{~b}}$ & n.p. ${ }^{c}$ & {$[12,13,53-56]$} \\
\hline 10 & 18.89 & Petunidin-3-acetylglucoside & $\mathrm{C}_{24} \mathrm{H}_{25} \mathrm{O}_{13}$ & 521.1296 & 0.2 & $317.07 * \mathrm{~b}, 302.04$ & n.p. ${ }^{c}$ & {$[42,46]$} \\
\hline 11 & 19.21 & Malvidin-3-glucoside & $\mathrm{C}_{23} \mathrm{H}_{25} \mathrm{O}_{12}$ & 493.1354 & 1.6 & $\begin{array}{l}331.08^{* b}, 315.05,287.06, \\
149.03\end{array}$ & n.p. ${ }^{c}$ & {$[42,45,47]$} \\
\hline 12 & 19.57 & Malvidin-3-(caffeoyl) glucoside & $\mathrm{C}_{32} \mathrm{H}_{31} \mathrm{O}_{15}$ & 655.1663 & 0.0 & $331.08^{* \mathrm{~b}}, 242.06,147.05$ & n.p. ${ }^{c}$ & {$[42,46]$} \\
\hline 13 & 19.77 & Peonidin-3-glucoside & $\mathrm{C}_{22} \mathrm{H}_{23} \mathrm{O}_{11}$ & 463.1239 & -0.2 & $\begin{array}{l}301.07^{* \mathrm{~b}}, 286.05,258.05, \\
201.06\end{array}$ & n.p. ${ }^{c}$ & {$[42,45,46]$} \\
\hline 14 & 19.92 & Peonidin-3-(caffeoyl) glucoside & $\mathrm{C}_{31} \mathrm{H}_{29} \mathrm{O}_{14}$ & 625.1551 & -1.0 & $301.07^{* \mathrm{~b}}, 149.02$ & n.p.c & {$[42,46]$} \\
\hline 15 & 20.56 & Malvidin-3-glucoside-pyruvic acid (Vitisin A) & $\mathrm{C}_{26} \mathrm{H}_{25} \mathrm{O}_{14}$ & 561.1244 & -4.1 & $399.08^{* b}$ & n.p. ${ }^{c}$ & {$[13,53-56]$} \\
\hline 16 & 20.73 & Petunidin-3-( $p$-coumaroyl) glucoside & $\mathrm{C}_{31} \mathrm{H}_{29} \mathrm{O}_{14}$ & 625.1567 & 1.6 & $317.07^{* \mathrm{~b}}$ & n.p. ${ }^{c}$ & {$[42,46]$} \\
\hline
\end{tabular}




\begin{tabular}{|c|c|c|c|c|c|c|c|c|}
\hline $\begin{array}{l}\text { Compound } \\
\text { number }^{\mathrm{a}}\end{array}$ & $t_{R}(\min )$ & Identity & $\begin{array}{l}\text { Molecular } \\
\text { formula }[\mathrm{M}]^{+}\end{array}$ & $\begin{array}{l}\text { Experimental } \\
\text { mass }(m / z)\end{array}$ & Error (ppm) & MSE fragments & MS/MS fragments & References \\
\hline 17 & 21.17 & Cyanidin-3-(p-coumaroyl) glucoside & $\mathrm{C}_{30} \mathrm{H}_{27} \mathrm{O}_{13}$ & 595.1458 & 1.0 & $287.06^{* \mathrm{~b}}, 149.02$ & n.p. ${ }^{c}$ & {$[42,46]$} \\
\hline 18 & 22.50 & Petunidin-3-(caffeoyl) glucoside & $\mathrm{C}_{31} \mathrm{H}_{29} \mathrm{O}_{15}$ & 641.1524 & 2.8 & $317.07 * \mathrm{~b}$ & n.p. ${ }^{c}$ & {$[42,46]$} \\
\hline 19 & 22.82 & Delphinidin-3-( $p$-coumaroyl)glucoside & $\mathrm{C}_{30} \mathrm{H}_{27} \mathrm{O}_{14}$ & 611.1413 & 2.0 & $303.04^{* b}$ & n.p. ${ }^{c}$ & {$[42,46]$} \\
\hline 20 & 22.87 & Delphinidin-3-acetylglucoside & $\mathrm{C}_{23} \mathrm{H}_{23} \mathrm{O}_{13}$ & 507.1139 & 0.0 & $303.05^{* \mathrm{~b}}$ & n.p. ${ }^{c}$ & {$[42,46]$} \\
\hline 21 & 23.46 & Petunidin-3-glucoside & $\mathrm{C}_{22} \mathrm{H}_{23} \mathrm{O}_{12}$ & 479.1191 & 0.2 & $317.07^{* \mathrm{~b}}$ & n.p. ${ }^{c}$ & {$[42,45-47]$} \\
\hline 22 & 24.00 & Cyanidin-3-glucoside & $\mathrm{C}_{21} \mathrm{H}_{21} \mathrm{O}_{11}$ & 449.1083 & -0.2 & $287.06^{* b}$ & n.p. ${ }^{c}$ & {$[42,45-47]$} \\
\hline 23 & 27.23 & Delphinidin-3-glucoside & $\mathrm{C}_{21} \mathrm{H}_{21} \mathrm{O}_{12}$ & 465.1046 & 2.8 & $303.05^{* b}$ & n.p. ${ }^{c}$ & {$[42,45-47]$} \\
\hline \multicolumn{9}{|c|}{ Red cabbage } \\
\hline 1 & 16.24 & Cyanidin-3-(sinapoyl) glucoside-5-glucoside & $\mathrm{C}_{38} \mathrm{H}_{41} \mathrm{O}_{20}$ & 817.2172 & -2.3 & $449.75,287.06^{* b}$ & $919,449,287^{* \mathrm{~b}}, 207,158,147$ & {$[45,58]$} \\
\hline 2 & 17.17 & $\begin{array}{l}\text { Cyanidin-3-(sinapoyl) } \\
\text { (sinapoyl)diglucoside-5-glucoside }\end{array}$ & $\mathrm{C}_{55} \mathrm{H}_{61} \mathrm{O}_{29}$ & 1185.3298 & 0.6 & $1023.28,449.10,287.06^{* b}$ & $\begin{array}{l}\text { 1023.29, 449.11, 287.06*b } 207.07 \\
177.06\end{array}$ & {$[45,58]$} \\
\hline 3 & 17.32 & $\begin{array}{l}\text { Cyanidin-3-(sinapoyl) } \\
\text { (feruloyl)diglucoside-5-glucoside }\end{array}$ & $\mathrm{C}_{54} \mathrm{H}_{59} \mathrm{O}_{28}$ & 1155.3190 & -0.3 & $\begin{array}{l}\text { 993.27, 899.45, 449.11, } \\
287.06^{* \mathrm{~b}}\end{array}$ & $\begin{array}{l}\text { 993.27, 449.11, 287.06*b } 207.07 \\
\text { 177.06 }\end{array}$ & {$[45,58]$} \\
\hline 4 & 17.87 & $\begin{array}{l}\text { Cyanidin-3-(feruloyl) } \\
\text { (feruloyl)diglucoside-5-glucoside }\end{array}$ & $\mathrm{C}_{53} \mathrm{H}_{57} \mathrm{O}_{27}$ & 1125.3094 & 0.6 & $963.26,449.11,287.06$ *b & $\begin{array}{l}963.27,449.11,287.06^{* \mathrm{~b}}, 207.07 \\
177.06\end{array}$ & {$[45,58]$} \\
\hline 5 & 22.86 & Cyanidin-3-(feruloyl) diglucoside-5-glucoside & $\mathrm{C}_{43} \mathrm{H}_{49} \mathrm{O}_{24}$ & 949.2615 & 0.1 & $787.20,449.11,287.06^{* b}$ & $\begin{array}{l}\text { 787.21, 449.11, 287.06*b }, 177.06 \\
145.03\end{array}$ & {$[45,58]$} \\
\hline 6 & 22.87 & Cyanidin-3-(sinapoyl) diglucoside-5-glucoside & $\mathrm{C}_{44} \mathrm{H}_{51} \mathrm{O}_{25}$ & 979.2717 & -0.2 & $449.11,287.06^{* b}$ & $\begin{array}{l}\text { 817.22, 449.11, 287.06*b }, 207.07 \text {, } \\
\text { 175.04 }\end{array}$ & {$[45,58]$} \\
\hline 7 & 23.29 & $\begin{array}{l}\text { Cyanidin-3-( } p \text {-coumaroyl) } \\
\text { diglucoside-5-glucoside }\end{array}$ & $\mathrm{C}_{42} \mathrm{H}_{47} \mathrm{O}_{23}$ & 919.2502 & -0.7 & $757.20,449.11,287.06^{* b}$ & $757.20,449.11,287.06^{* \mathrm{~b}}, 147.05$ & {$[45,58]$} \\
\hline 8 & 25.20 & $\begin{array}{l}\text { Cyanidin-3-(sinapoyl) } \\
\text { (feruloyl)triglucoside-5-glucoside }\end{array}$ & $\mathrm{C}_{60} \mathrm{H}_{69} \mathrm{O}_{33}$ & 1317.3732 & 0.8 & $1155.33,449.11,287.06^{* b}$ & $1155.32,449.11,287.06^{* b}, 177.05$ & [58] \\
\hline 9 & 25.27 & $\begin{array}{l}\text { Cyanidin-3-(sinapoyl) } \\
\text { (sinapoyl)triglucoside-5-glucoside }\end{array}$ & $\mathrm{C}_{61} \mathrm{H}_{71} \mathrm{O}_{34}$ & 1347.3806 & -1.6 & $1185.32,449.11,287.06^{* b}$ & 1185.36, 449.11, 287.06*b 177.05 & [58] \\
\hline 10 & 25.54 & $\begin{array}{l}\text { Cyanidin-3-(sinapoyl) ( } p \text {-coumaroyl) } \\
\text { triglucoside-5-glucoside }\end{array}$ & $\mathrm{C}_{59} \mathrm{H}_{67} \mathrm{O}_{32}$ & 1287.3625 & 0.8 & $1125.28,449.11,287.06^{* b}$ & $1125.30,449.11,287.06^{* b}, 147.05$ & [58] \\
\hline 11 & 25.69 & Cyanidin-3-(caffeoyl) diglucoside-5-glucoside & $\mathrm{C}_{42} \mathrm{H}_{47} \mathrm{O}_{24}$ & 935.2427 & -3.2 & $773.19,449.11,287.06^{* b}$ & n.p. ${ }^{c}$ & {$[45,58]$} \\
\hline 12 & 27.91 & Cyanidin-3-(feruloyl) triglucoside-5-glucoside & $\mathrm{C}_{49} \mathrm{H}_{59} \mathrm{O}_{29}$ & 1111.3146 & 0.4 & $949.16,449.11,287.06^{* b}$ & $947.24,449.11,287.06^{* \mathrm{~b}}, 116.05$ & {$[45,58]$} \\
\hline 13 & 28.17 & Cyanidin-3-(sinapoyl) triglucoside-5-glucoside & $\mathrm{C}_{50} \mathrm{H}_{61} \mathrm{O}_{30}$ & 1141.3247 & -0.1 & $979.26,449.11,287.06^{* b}$ & $979.26,449.11,287.06^{* b}, 207.07$ & {$[45,58]$} \\
\hline 14 & 28.99 & Cyanidin-3-diglucoside-5-glucoside & $\mathrm{C}_{33} \mathrm{H}_{41} \mathrm{O}_{21}$ & 773.2141 & 0.1 & $611.16,449.11,287.06^{* b}$ & n.p. ${ }^{c}$ & {$[45,58]$} \\
\hline 15 & 31.23 & Cyanidin-3-(feruloyl) triglucoside-5-glucoside & $\mathrm{C}_{49} \mathrm{H}_{59} \mathrm{O}_{29}$ & 1111.3127 & -1.3 & $949.25,449.11,287.06^{* b}$ & $949.25,449.11,287.06^{* b}, 177.05$ & {$[45,58]$} \\
\hline 16 & 31.29 & Cyanidin-3-(sinapoyl) triglucoside-5-glucoside & $\mathrm{C}_{50} \mathrm{H}_{61} \mathrm{O}_{30}$ & 1141.3220 & -0.4 & $979.27,449.11,287.06^{* b}$ & $\begin{array}{l}979.28,449.11,287.06^{* b}, 207.06, \\
177.05\end{array}$ & {$[45,58]$} \\
\hline 17 & 31.71 & $\begin{array}{l}\text { Cyanidin-3-( } p \text {-coumaroyl }) \\
\text { triglucoside-5-glucoside }\end{array}$ & $\mathrm{C}_{48} \mathrm{H}_{57} \mathrm{O}_{28}$ & 1081.3055 & 1.8 & $919.25,449.11,287.06^{* b}$ & n.p.c & \\
\hline \multicolumn{9}{|l|}{ Red radish } \\
\hline 1 & 9.31 & Cyanidin-3-(glycosyl) rhamnoside & $\mathrm{C}_{27} \mathrm{H}_{31} \mathrm{O}_{15}$ & 595.1678 & 2.5 & $433.11,287.06^{* b}$ & n.p. ${ }^{c}$ & [45] \\
\hline 2 & 12.83 & $\begin{array}{l}\text { Pelaragonin-3-(feruloyl) } \\
\text { (feruloyl)diglucoside-5-(malonoyl)glucoside }\end{array}$ & $\mathrm{C}_{56} \mathrm{H}_{59} \mathrm{O}_{29}$ & 1195.3137 & -0.4 & $\begin{array}{l}519.11,271.06^{* b}, 177.05 \\
149.02\end{array}$ & n.p. ${ }^{c}$ & [45] \\
\hline 3 & 13.11 & $\begin{array}{l}\text { Pelaragonin-3-(p-coumaroyl) } \\
\text { (feruloyl)diglucoside-5-(malonoyl)glucoside }\end{array}$ & $\mathrm{C}_{55} \mathrm{H}_{57} \mathrm{O}_{28}$ & 1165.3037 & 0.1 & $917.23,519.11,271.06^{* b}$ & n.p. ${ }^{c}$ & [45] \\
\hline 4 & 15.27 & Pelaragonin-3-glucoside-5-glucoside & $\mathrm{C}_{27} \mathrm{H}_{31} \mathrm{O}_{15}$ & 595.1687 & 4.0 & $271.06^{* b}$ & n.p. ${ }^{c}$ & [45] \\
\hline 5 & 15.25 & $\begin{array}{l}\text { Pelaragonin-3-(feruloyl) } \\
\text { diglucoside-5-(malonoyl) glucoside }\end{array}$ & $\mathrm{C}_{46} \mathrm{H}_{51} \mathrm{O}_{26}$ & 1019.2676 & 0.7 & $771.22,519.12,271.06 *$ b & $771.22,519.12,271.06^{* \mathrm{~b}}, 177.05$ & [45] \\
\hline 6 & 15.64 & $\begin{array}{l}\text { Pelaragonin-3-( } p \text {-coumaroyl)diglucoside-5- } \\
\text { (malonoyl)glucoside }\end{array}$ & $\mathrm{C}_{45} \mathrm{H}_{49} \mathrm{O}_{25}$ & 989.2566 & 0.3 & $741.21,519.12,271.06^{* b}$ & $741.21,519.12,271.06^{* b}, 147.05$ & [45] \\
\hline 7 & 16.78 & $\begin{array}{l}\text { Pelaragonin-3-(feruloyl) } \\
\text { diglucoside-5-(malonoyl) glucoside }\end{array}$ & $\mathrm{C}_{46} \mathrm{H}_{51} \mathrm{O}_{26}$ & 1019.2663 & -0.6 & $519.11,271.06^{* b}$ & $519.11,271.06^{* b}, 147.05$ & [45] \\
\hline 8 & 16.81 & Cyanidin-3-(diglucosyl)rhamnoside & $\mathrm{C}_{33} \mathrm{H}_{41} \mathrm{O}_{20}$ & 757.2194 & 0.4 & $287.06^{* \mathrm{~b}}$ & $287.06^{* \mathrm{~b}}, 203.03$ & [45] \\
\hline 9 & 17.04 & $\begin{array}{l}\text { Pelaragonin-3-( } p \text {-coumaroyl)diglucoside-5- } \\
\text { (malonoyl)glucoside }\end{array}$ & $\mathrm{C}_{45} \mathrm{H}_{49} \mathrm{O}_{25}$ & 989.2567 & 0.4 & 741.19, 519.11, 271.06*b & $741.19,519.11,271.06^{* b}, 201.06$ & [45] \\
\hline 10 & 17.45 & $\begin{array}{l}\text { Pelaragonin-3-(feruloyl) } \\
\text { diglucoside-5-(malonoyl) glucoside }\end{array}$ & $\mathrm{C}_{46} \mathrm{H}_{51} \mathrm{O}_{26}$ & 1019.2662 & -0.7 & $\begin{array}{l}771.21,519.11,271.06^{* b} \\
149.02\end{array}$ & $771.21,519.11,271.06^{* \mathrm{~b}}, 147.05$ & [45] \\
\hline
\end{tabular}




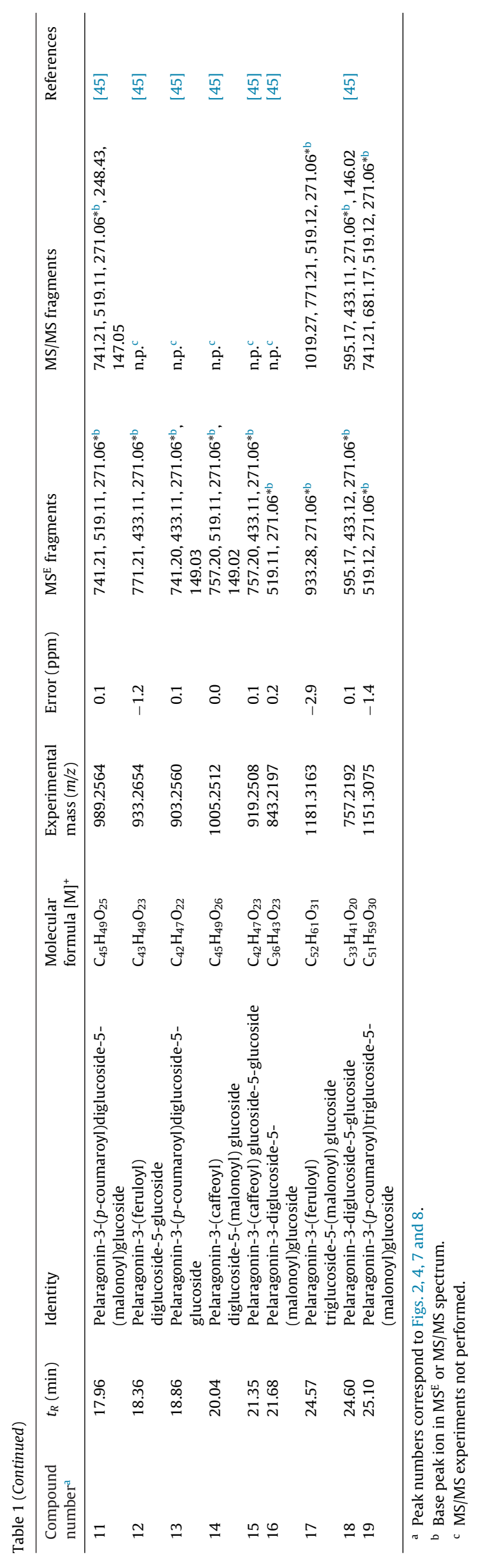

identification in the current work was therefore based on previous literature reports for the relevant samples [46,48-52].

\subsubsection{HILIC analysis of black bean, blueberry and red grape skin anthocyanins}

HILIC-UV $(500 \mathrm{~nm})$ chromatograms obtained using the optimised (Section 2.3.2) gradient conditions for black bean, blueberry and red grape skin anthocyanins are presented in Fig. 7, with compound numbers corresponding to Table 1. Mainly anthocyanidin-3-O-monoglycosides were found in blueberries and black beans, while red grape skins also contained several acylated derivatives. A total of 35 anthocyanins were identified in these samples, most of which have previously been reported in the literature [43-46]. Identification of compounds was relatively straightforward based on accurate mass data as well as fragmentation information (Table 1). Due to the retention mechanism in HILIC, anthocyanidin-galactosides could not be distinguished from their-glucoside isomers, although the former have previously been identified in black beans and blueberries [44-46].

In red grape skins, it is interesting to note the presence of several derived anthocyanin species, including the pyruvic acid (Vitisin A (15)) [53] and acetaldehyde (Vitisin B (3) ) [54] derivatives of malvidin-3-O-glucoside, as well as malvidin-3-acetylglucoside (acetylvitisin A (6) and acetylvitisin B (1)) and malvidin-3-pcoumaroylglucoside (coumaroylvitisin A (9) and coumaroylvitisin B (2)) [13,53-56]. These compounds are formed by reaction between the relevant anthocyanin and pyruvic acid and acetaldehyde, respectively, and are mainly thought to be produced during fermentation and wine ageing $[13,53,54]$. Our results confirm a previous report of Vitisin A in grape skin extracts [55], although to the best of our knowledge, this is the first time that acetylvitisin A, coumaroylvitisin A and the B-type Vitisins are detected in grape skin. These compounds displayed lower retention than the 'native' anthocyanins in HILIC due to the reduced polarity of the derived anthocyanidin base.

\subsubsection{HILIC analysis of red cabbage and red radish anthocyanins}

Chromatograms recorded at $500 \mathrm{~nm}$ for the HILIC analysis of red cabbage and red radish anthocyanins using optimised gradient conditions (Section 2.3.2) are shown in Fig. 8 (compound numbers correspond to Table 1 ).

A total of 17 anthocyanins were identified in red cabbage (Fig. 8a, Table 1). Only cyanidin derivatives were found in this vegetable, with variation stemming from different acylation patterns of cyanidin-3-di/triglucoside-5-glucoside [46,52,57]. The general elution pattern was therefore based on the number of glycosydic groups and the type and number of acylated groups.

Several compounds with identical molecular weights were detected in red cabbage, including cyanidin-3-(sinapoyl) triglucoside-5-glucoside (13 and 16) and cyanidin-3-(feruloyl) triglucoside-5-glucoside (12 and 15). Although it is not possible to unambiguously identify these compounds based on the available MS data, their structures most likely differ in terms of the position of acylation of the -sinapoyl/-feruloyl groups. Other acylated cyanidin-3-triglucoside-5-glucoside derivatives detected include cyanidin-3-(sinapoyl)(feruloyl)triglucoside-5-glucoside (8), cyanidin-3-(sinapoyl)(sinapoyl)triglucoside-5-glucoside (9) and cyanidin-3-(sinapoyl)( $p$-coumaroyl)triglucoside-5-glucoside (10) [58]. These compounds generally showed lower retention in HILIC than the corresponding non-acylated cyanidin-3diglucoside-5-glucoside (14).

Compound 17 has have similar MS characteristics, both in terms of (nominal) molecular weight and fragmentation pattern, to cyanidin-3-(caffeoyl)( $p$-coumaroyl)diglucoside-5-glucoside previously identified in red cabbage [46,58]. However, accurate mass data clearly indicated that the molecular weight of compound $\mathbf{1 7}$ 

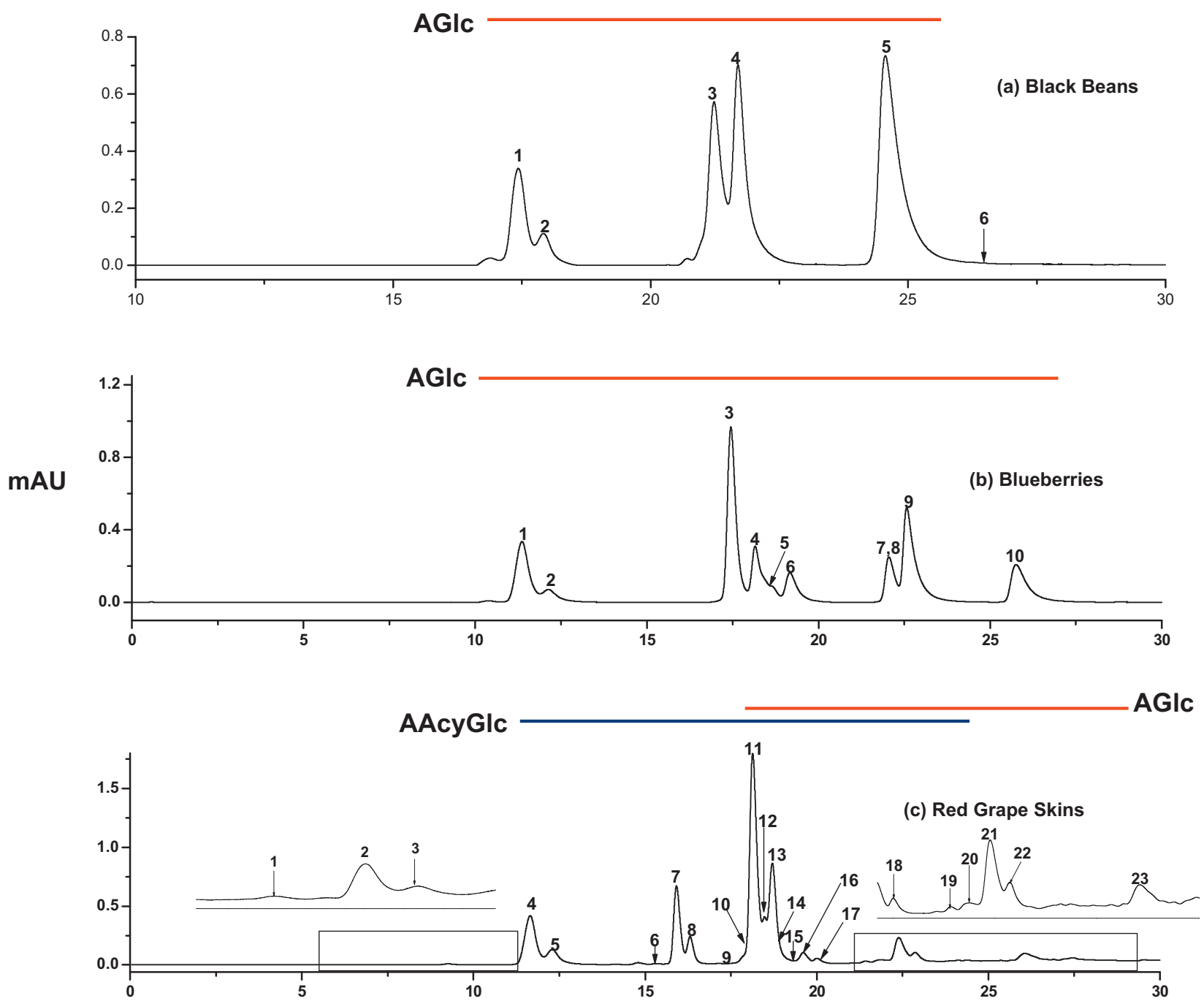

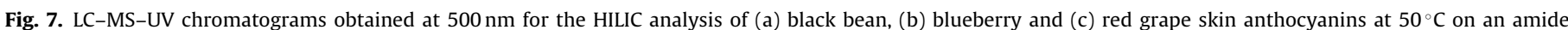

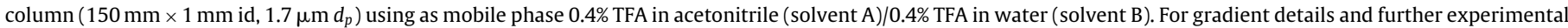

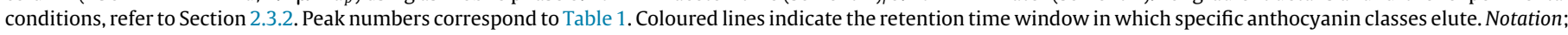
AGlc: anthocyanidin-glycosides, AAcyGlc: anthocyanidin-acyl-glycosides.

did not fit the structure of the latter compound, but rather that of cyanidin-3-( $p$-coumaroyl)triglucoside-5-glucoside. Distinction between these structures would not be possible based on lowresolution MS or MS/MS data alone, even considering the relative retention times, which clearly highlights the benefits of high resolution MS (HR-MS) instruments for anthocyanin analysis.

Nineteen anthocyanins were identified in red radish (Fig. 8b, Table 1), mainly comprising highly acylated anthocyanins derivatives of pelaragonidin (the exceptions being cyanidin-3(glycosyl)rhamnoside (1) and cyanidin-3-(diglycosyl)rhamnoside (8), the latter reported here in red radish for the first time). The principal anthocyanins in this sample are acylated derivatives of pelaragonin-3-diglucoside-5-glucoside, with the major acylsubstituents being a combination of - $p$-coumaroyl, -feruloyl, and -malonoyl groups [59].

As was the case for red cabbage, several isomeric structures were detected, including pelaragonin-3-(feruloyl)diglucoside5-(malonoyl)glucoside (5, 7 and 10) and pelaragonin-3-( $p$ coumaroyl)diglucoside-5-(malonoyl)glucoside (6,9 and 11). All isomers displayed identical fragmentation patterns, and could therefore not be unambiguously identified based solely on MS data, although they most likely differ in terms of their respective acylation patterns.
Pelaragonidin-3-(feruloyl)triglucoside-5-(malonoyl)glucoside (17) and pelaragonin-3-( $p$-coumaroyl) triglucoside-5-(malonoyl)glucoside (19) are to the best of our knowledge reported here for the first time in red radish. Similar to red cabbage, compounds with similar MS properties were previously assigned to the 3-(feruloyl)(caffeoyl)diglucoside and 3-( $p$ coumaroyl)(caffeoyl)diglucoside derivatives [46]. While low resolution MS cannot distinguish between -(caffeoyl)diglucoside and triglucoside substitution, HR-MS data confirm the structure containing the latter. All other compounds identified in red radish (Table 1) were in agreement with previously reported literature [35].

\subsection{Observations on the retention behaviour of anthocyanins in HILIC}

From the data presented in Figs. 7 and 8 and Table 1, some interesting observations may be made regarding the retention behaviour of anthocyanins in HILIC. First, for the same glycosylation and acylation pattern, compounds are separated according to the polarity of the anthocyanidin base; the elution order is therefore malvidin (with most methoxy-substituents), followed by peonidin, petunidin, cyanidin and lastly delphinidin. Pelaragonidin 

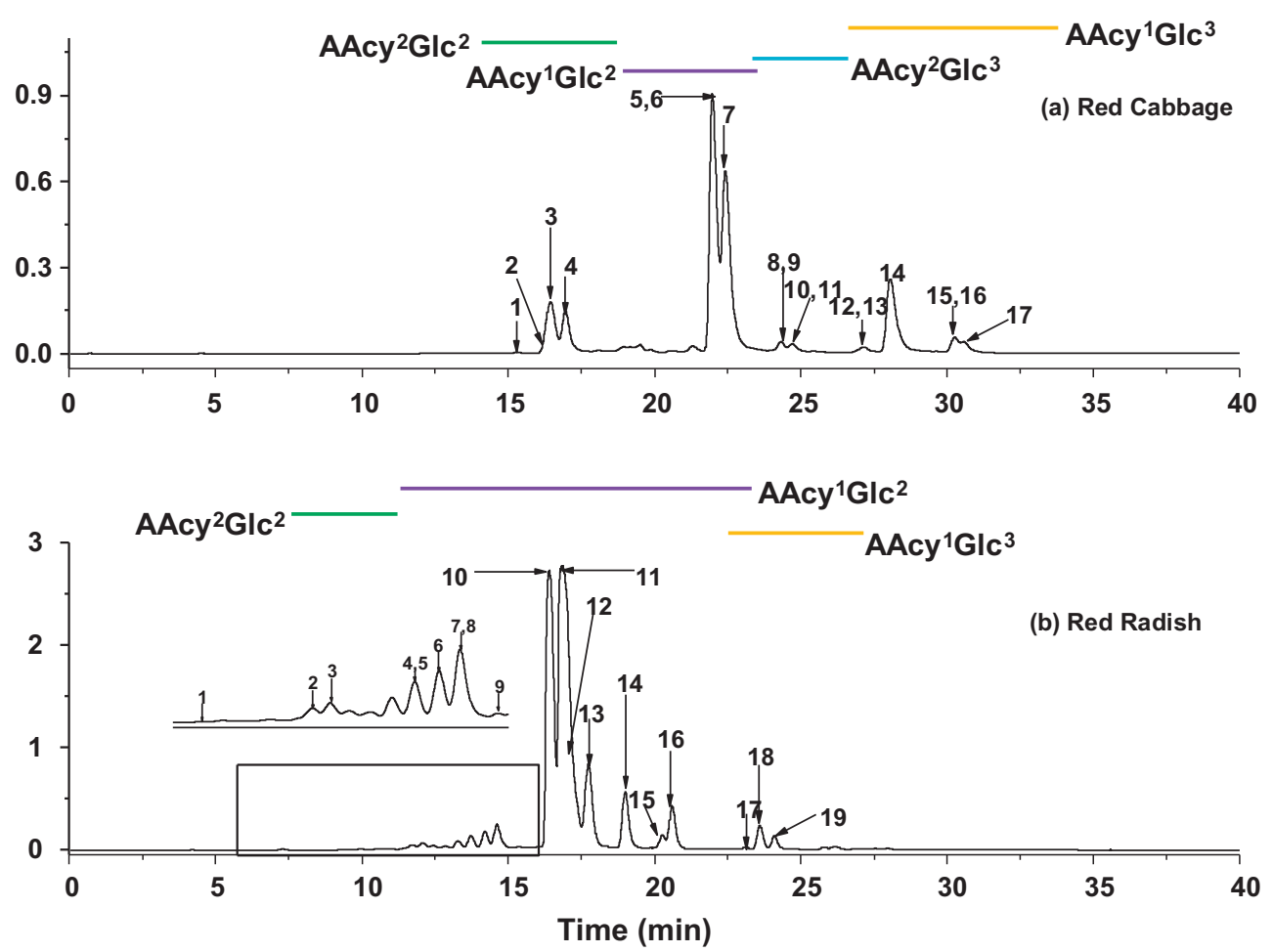

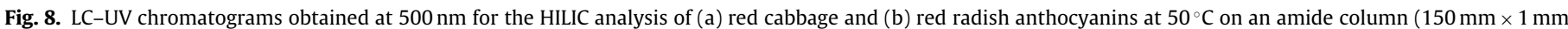

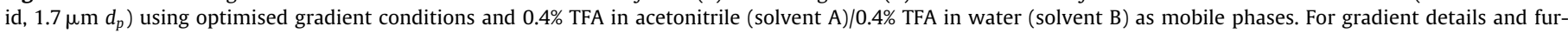

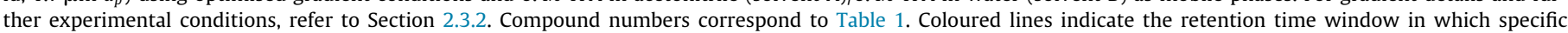

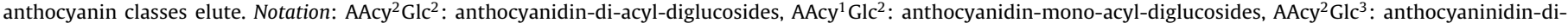
acyl-triglucosides, $\mathrm{AAcy}^{1} \mathrm{Glc}^{3}$ : anthocyanidin-mono-acyl-triglucosides.

derivatives eluted earlier than the corresponding cyanidin derivatives (compare compounds $\mathbf{1 4}$ and $\mathbf{1 8}$ in red cabbage and radish, respectively), although their retention relative to the other anthocyanidins is not clear due to the lack of compounds containing identical glycosylation/acylation substituents.

In terms of the effect of glycosylation on HILIC retention, arabinosides eluted before the hexosides for the same anthocyanidin base and degree of acylation (which is the opposite elution order compared to RP-LC), while glucosides and galactosides were not resolved in HILIC. In contrast, galactosides are separated from glucosides in RP-LC, with the latter eluting later and followed by the arabinosides [44].

A higher degree of glycosylation results in higher HILIC retention [27]. The elution order of anthocyanidin-mono-glycosides, as observed in blueberries and black beans, is therefore: malvidin3 -arabinoside $\leq$ malvidin-3-glucoside $\leq$ peonidin-3-arabinoside $\leq$ peonidin-3-glucoside $\leq$ petunidin-3-arabinoside $\leq$ petunidin3-glucoside $\leq$ cyanidin-3-arabinoside $\leq$ cyanidin-3-glucoside $\leq$ cyanidin-3-glucoside $\leq$ delphinidin-3-arabinoside $\leq$ delphinidin3-glucoside.

Acylation of anthocyanins generally decreases HILIC retention, the exception being caffeoyl-derivatives, which show higher retention than the corresponding non-acylated anthocyanin. Mono-acylated anthocyanins eluted in the sequence acetylglucosides $<p$-coumaroyl-glucosides $<$ glucosides $<$ caffeoyl-gluco sides (as observed in grape skins). It is also relevant to note that for anthocyanins acylated with cinnamic acids, cis and trans isomers were not separated in HILIC, whereas the former isomers elute earlier in RP-LC [20,58].

For highly acylated compounds, the evaluation of the effect of individual acyl groups on HILIC retention is far from straightforward, partially since this will also depend on the substitution pattern. Nevertheless, some conclusions may be drawn from the retention order observed for related compounds. For example, the relative retention of the acylated derivatives of cyanidin-3diglucoside-5-glucoside in red cabbage are according to the polarity of the respective acyl groups: sinapoyl-sinapoyl $\leq$ sinapoylferuloyl $\leq$ feruloyl-feruloyl $\leq$ feruloyl $\leq$ sinapoyl $\leq p$-coumaroyl $\leq$ caffeoyl. The same elution order is observed for the more retained acylated cyanidin-3-triglucoside-5-glucoside derivatives, as well as for the acylated derivatives of pelaragonin-3diglucoside-5-(malonyl)glucoside observed in red radish: feruloyl-feruloyl $\leq(p$-coumaroyl $)($ feruloyl $) \leq$ feruloyl $\leq p$-couma royl $\leq$ caffeoyl (Table 1 ).

Comparison of the abovementioned HILIC retention orders with well-established RP-LC elution characteristics of anthocyanins $[12,14,17,18,43-46,58]$ highlights the complementary nature of the two separation modes. This opens the door for the combination of HILIC and RP-LC separations for the comprehensive 2-dimensional liquid chromatographic (LC $\times$ LC) analysis of anthocyanins. Due to the divergent retention mechanisms, improved resolution of complex anthocyanin fractions by HILIC $\times$ RP-LC analysis may be envisaged. Several approaches may be used to overcome the challenges associated with the coupling of HILIC and RP-LC due to mobile phase incompatibility, as illustrated in reports highlighting the beneficial application of HILIC $\times$ RP-LC for the high-resolution separation of phenolic samples [25-27,60].

\section{Conclusions}

The application of HILIC for the analysis of anthocyanins has been demonstrated for the first time. Due to the unique chromatographic behaviour of anthocyanins, extensive optimisation was required to deliver acceptable performance. This involved first of all the selection of the most suitable stationary phase (the $1.7 \mu \mathrm{m}$ $\mathrm{BEH}$ amide phase). Highly acidic mobile phases are especially 
critical when using high organic content mobile phases, due to the pH-dependant solution chemistry of anthocyanins. The use of elevated temperatures and analysis at low flow rates further improved the separation of these compounds. HILIC methods suitable for the analysis of a diverse range of anthocyanins, including anthocyanidin-mono-, di- and tri-glycosides and their acylated derivatives were developed. Application to blueberries, red grape skins, black beans, red cabbage and red radish illustrates the potential of the technique, and allowed identification of 71 anthocyanins by ESI-MS(/MS).

HILIC was found to offer an alternative separation mechanism to RP-LC, with the clear separation according to degree of glycosylation and acylation facilitating tentative compound identification. HILIC has the added advantage of lower operating pressures due to the lower viscosity of the eluents. Co-elution of isomeric anthocyanidin-hexosides and cis/trans acylated isomers as well as lower chromatographic efficiency and higher organic modifier consumption represent drawbacks of HILIC compared to RP-LC. The alternative separation mechanism offered by HILIC shows promise for use in 2-dimensional LC analysis of complex anthocyanin fractions, which will be explored in future work. Furthermore, the applicability of the developed methods for the simultaneous analysis of anthocyanins and non-coloured flavonoids should be explored.

\section{Acknowledgements}

The authors gratefully acknowledge Sasol (grant to AdV and bursary to CW), the National Research Foundation (NRF, South Africa, Grant 81830 to AdV) and the International Foundation of Science (IFS, Sweden, Grant F/4904-1 to AdV) for financial support. W.J. Gerber is thanked for helpful discussions.

\section{Appendix A. Supplementary data}

Supplementary material related to this article can be found, in the online version, at http://dx.doi.org/10.1016/j.chroma. 2013.10.045.

\section{References}

[1] W. Yi, C.C. Akoh, J. Fischer, G. Krewer, J. Agric. Food Chem. 54 (2006) 5651.

[2] M.E. Olsson, K.-E. Gustavsson, S. Anderson, A. Nilsson, R.-D. Duan, J. Agric. Food Chem. 52 (2004) 7264.

[3] H.A. Hassan, A.F. Abdel-Aziz, Food Chem. Toxicol. 48 (2010) 1999.

[4] D. Strack, V. Wray, J.B. Harborne (Eds.), Flavonoids: Advances in Research since 1986, Chapman and Hall, London, 1994 (chapter 1).

[5] J. Valls, S. Millán, M.P. Martí, E. Borràs, L. Arola, J. Chromatogr. A 1216 (2009) 7143.

[6] Ø.M. Andersen, M.Jordheim, The anthocyanin, in: Ø.M. Andersen, K.R. Markham (Eds.), Flavonoids: Chemistry, Biochemistry and Applications, CRC Press, Boca Raton, 2006, p. 471

[7] C.R. Welch, Q. Wu, J.E. Simon, Curr. Anal. Chem. 4 (2008) 75

[8] Ø.M. Andersen, Recent advances in the field of anthocyanins, in: V. Lattanzio, F. Daayf (Eds.), Polyphenols-Recent Advances in Research, Blackwell, London, 2008, p. 167

[9] E. Hebrero, C. Santos-Buelga, J.C. Rivas-Gonzalo, Am. J. Enol. Vitic. 39 (1988) 227.

[10] K.R. Maatta-Riihinen, A. Kamal-Eldin, P.H. Mattila, A.M. Gonzalez-Paramas, A.R. Torronen, J. Agric. Food Chem. 52 (2004) 4477.

[11] V. Hong, R.E. Wrolstad, J. Agric. Food Chem. 38 (1990) 708
[12] P. Alberts, M.A. Stander, A. de Villiers, J. Chromatogr. A 1235 (2012) 92.

[13] A. de Villiers, G. Vanhoenacker, P. Majek, P. Sandra, J. Chromatogr. A 1054 (2004) 195.

[14] B. Abad-García, L.A. Berrueta, S. Garmón-Lobato, B. Gallo, F. Vicente, J. Chromatogr. A 1216 (2009) 5398

[15] M.M. Giusti, L.E. Rodrıguez-Saona, D. Griffin, R.E. Wrolstad, J. Agric. Food Chem. 47 (1999) 4657

[16] J. Sun, L.-Z. Lin, P. Chen, Rapid Commun. Mass Spectrom. 26 (2012) 1123.

[17] A. de Villiers, D. Cabooter, F. Lynen, G. Desmet, P. Sandra, J. Chromatogr. A 1216 (2009) 3270

[18] A. de Villiers, D. Cabooter, F. Lynen, G. Desmet, P. Sandra, J. Chromatogr. A 1218 (2011) 4660.

[19] C. Vergara, C. Mardones, I. Hermosin-Gutierresz, D. von Baer, J. Chromatogr. A 1217 (2010) 5710.

[20] A.J. Alpert, J. Chromatogr. A 177 (1990) 499

[21] C.T. Mant, J.R. Litowski, R.S. Hodges, J. Chromatogr. A 816 (1998) 65

[22] P. Jandera, T. Hajek, J. Sep. Sci. 32 (2009) 3603.

[23] A. Yanagida, H. Murao, M. Ohnishi-Kameyama, Y. Yamakawa, A. Shoji, M Tagashira, T. Kanda, H. Shindo, Y. Shibusawa, J. Chromatogr. A 1143 (2007) 153.

[24] H. Zhang, Z. Guo, F. Zhang, Q. Xu, X. Liang, J. Sep. Sci. 31 (2008) 1623.

[25] T. Beelders, K.M. Kalili, E. Joubert, D. de Beer, A. de Villiers, J. Sep. Sci. 35 (2012) 1808.

[26] K.M. Kalili, A. de Villiers, J. Chromatogr. A 1216 (2009) 6274.

[27] K.M. Kalili, A. de Villiers, J. Sep. Sci. 33 (2010) 853.

[28] P. Jandera, J. Sep. Sci. 31 (2008) 1421.

[29] P. Jandera, Anal. Chim. Acta 692 (2011) 1.

[30] P. Jandera, T. Hajek, V. Skerıkova, J. Soukup, J. Sep. Sci. 33 (2010) 841.

[31] D.V. McCalley, J. Chromatogr. A 1171 (2007) 46.

[32] M.A. Kelm, J.C. Johnson, R.J. Robbins, J.F. Hammerstone, H.H. Schmitz, J. Agric. Food Chem. 54 (2006) 1571.

[33] L. Novakova, I. Kaufmannova, R. Janska, J. Sep. Sci. 33 (2010) 765.

[34] Y. Leydet, R. Gavara, V. Petrov, A.M. Diniz, A.J. Parola, J.C. Lima, F. Pina, Phytochemistry 83 (2012) 125

[35] J.A. Kennedy, A.L. Waterhouse, J. Chromatogr. A 866 (2000) 25.

[36] R. Brouillard, J.E. Dubois, J. Am. Chem. Soc. 99 (1976) 1359.

[37] R. Brouillard, B. Delaporte, J. Am. Chem. Soc. 99 (1977) 8461.

[38] R. Brouillard, J.-E. Dubois, J. Am. Chem. Soc. 99 (1977) 1359.

[39] L.G. Gagliardi, C.B. Castells, C. Rafols, M. Roses, E. Bosch, Anal. Chem. 79 (2007) 3180.

[40] P. Mazzuca, P. Ferranti, G. Picariello, L. Chianese, F. Addeo, J. Mass Spectrom. 40 (2005) 83.

[41] T. Ikegami, K. Tomomatsu, H. Takubo, K. Horie, N. Tanaka, J. Chromatogr. A 1184 (2008) 474.

[42] Y. Li, R. Ma, Z. Xu, J. Wang, T. Chen, F. Chen, Z. Wang, J. Sci. Food Agric. 93 (2013) 1404.

[43] A.A. De la Cruz, G. Hilbert, C. Riviere, V. Mengin, N. Ollat, L. Bordenave, S Decroocq, J.-C. Delaunay, S. Delrot, J.-M. Merillon, J.-P. Monti, E. Gomez, T. Richard, Anal. Chim. Acta 732 (2012) 145

[44] J.S. Barnes, H.P. Nguyen, S. Shen, K.A. Schug, J. Chromatogr. A 1216 (2009) 4728

[45] X. Wu, R.L. Prior, J. Agric. Food Chem. 53 (2005) 2589.

[46] X. Wu, R.L. Prior, J. Agric. Food Chem. 53 (2005) 3101

[47] J.S. Barnes, K.A. Schug, Int. J. Mass Spectrom. 308 (2011) 71.

[48] G.J. McDougal, S. Fyffe, P. Dobson, S. Stewart, Phytochem. 68 (2007) 1285.

[49] E. Idaka, K. Suzuki, H. Yaakita, T. Ogawa, T. Kondo, T. Goto, Chem. Lett. 1 (1987) 145.

[50] E. Idaka, H. Yamakita, T. Ogawa, T. Kondo, M. Yamamoto, T. Goto, Chem. Lett. 6 (1987) 1213

[51] K.K. Ikeda, H. Nakamura, M. Nakatani, N., Chem. Express 2 (1987) 563

[52] G. Lanzarini, L. Morselli, Ind. Conserv, 49 (1974) 16.

[53] J. Bakker, P. Bridle, T. Honda, H. Kuwano, N. Saito, N. Terahara, C.F. Timberlake Phytochem. 44 (1977) 1375

[54] J. Bakker, C.F. Timberlake, J. Agric. Food Chem. 45 (1997) 35

[55] N. Mateus, A.M.S. Silva, J. Vercauteren, V. de Freitas, J. Agric. Food Chem. 49 (2001) 4836.

[56] A.M. Vivar-Quintana, C. Santos-Buelga, J.C. Rivas-Gonzalo, Anal. Chim. Acta 458 (2002) 147.

[57] T. Shimizu, T. Muroi, T. Ichi, M. Nakamura, K. Yoshihira, J. Food Hyg. Soc. Japan 38 (1997) 34.

[58] P. Arapitsas, Per J.R. Sjober, C. Turner, Food Chem. 109 (2008) 219.

[59] H. Matsufuji, T. Otsuki, T. Takeda, M. Chino, M. Takeda, J. Agric. Food Chem. 51 (2003) 3157

[60] P. Jandera, T. Hájek, M. Stanková, K. Vynuchalová, P. Cesla, J. Chromatogr. A 1268 (2012) 91. 


\section{SUPPORTING INFORMATION FOR THE MANUSCRIPT:}

\section{Hydrophillic interaction chromatographic analysis of anthocyanins}

Chandré M. Willemse ${ }^{\mathrm{a}}$, Maria A. Stander ${ }^{\mathrm{b}, \mathrm{c}}$, André de Villiers ${ }^{\mathrm{a}}$

aStellenbosch University, Department of Chemistry and Polymer Science, Private Bag X1, Matieland 7602, South Africa

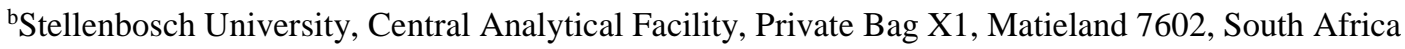

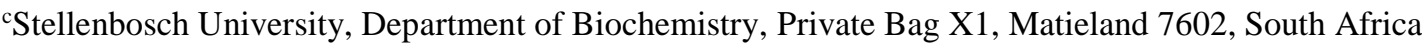




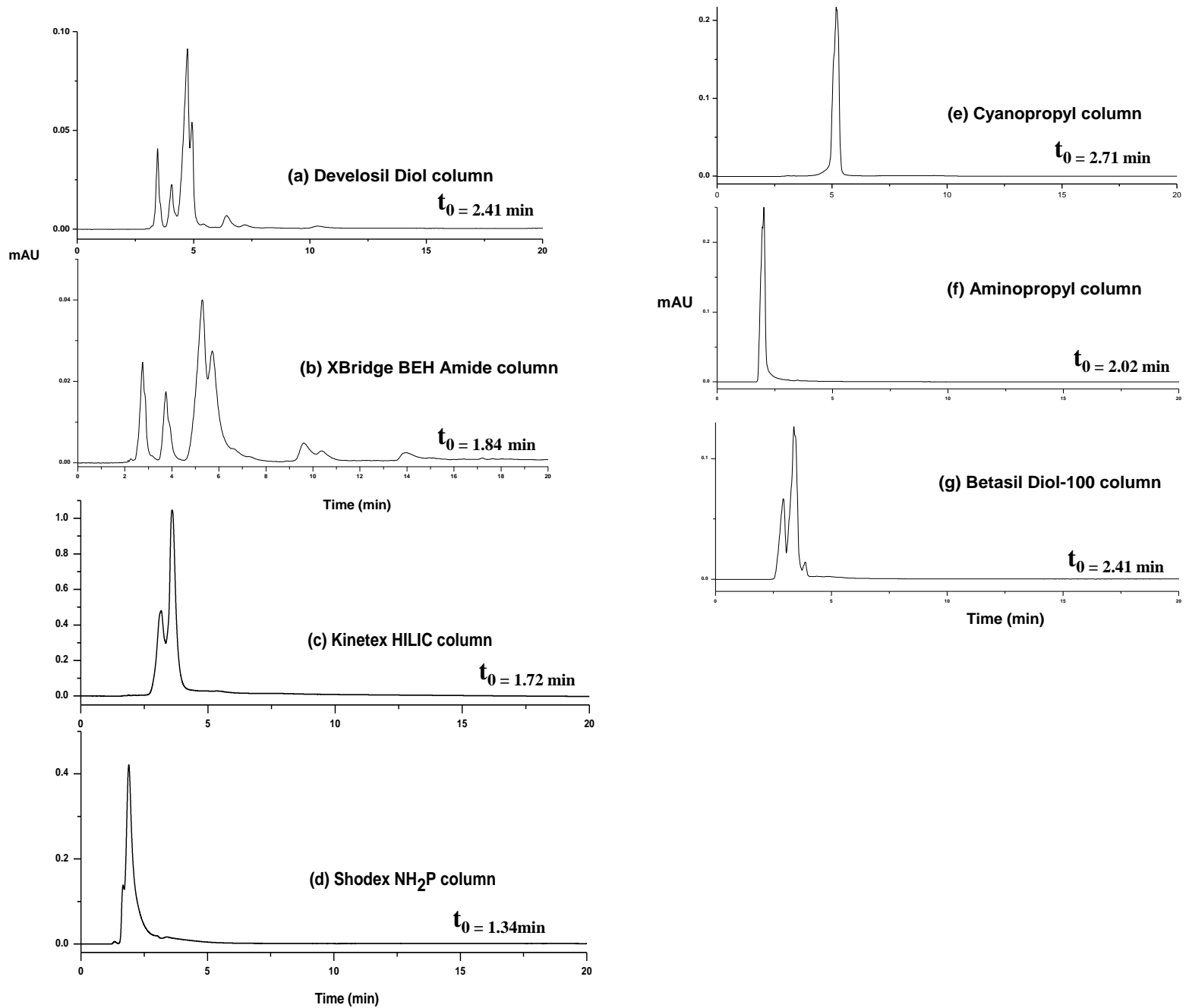

Figure S1: LC-UV chromatograms obtained at $500 \mathrm{~nm}$ for the HILIC analysis of grape skin anthocyanins on different HILIC stationary phases: (a) Develosil diol, (b) XBridge BEH Amide, (c) Kinetex HILIC (silica), (d) Shodex Asahipak $\mathrm{NH}_{2} \mathrm{P}$, (e) Luna cyanopropyl, (f) Spherisorb aminopropyl, and (g) Betasil Diol-100. Mobile phases: (A) $5 \%$ formic acid in water and (B) $5 \%$ formic acid in water. Analyses performed at ambient temperature. Injection volumes of $2 \mu \mathrm{L}$ were used. For further experimental detail, refer to section 2.3.1. 

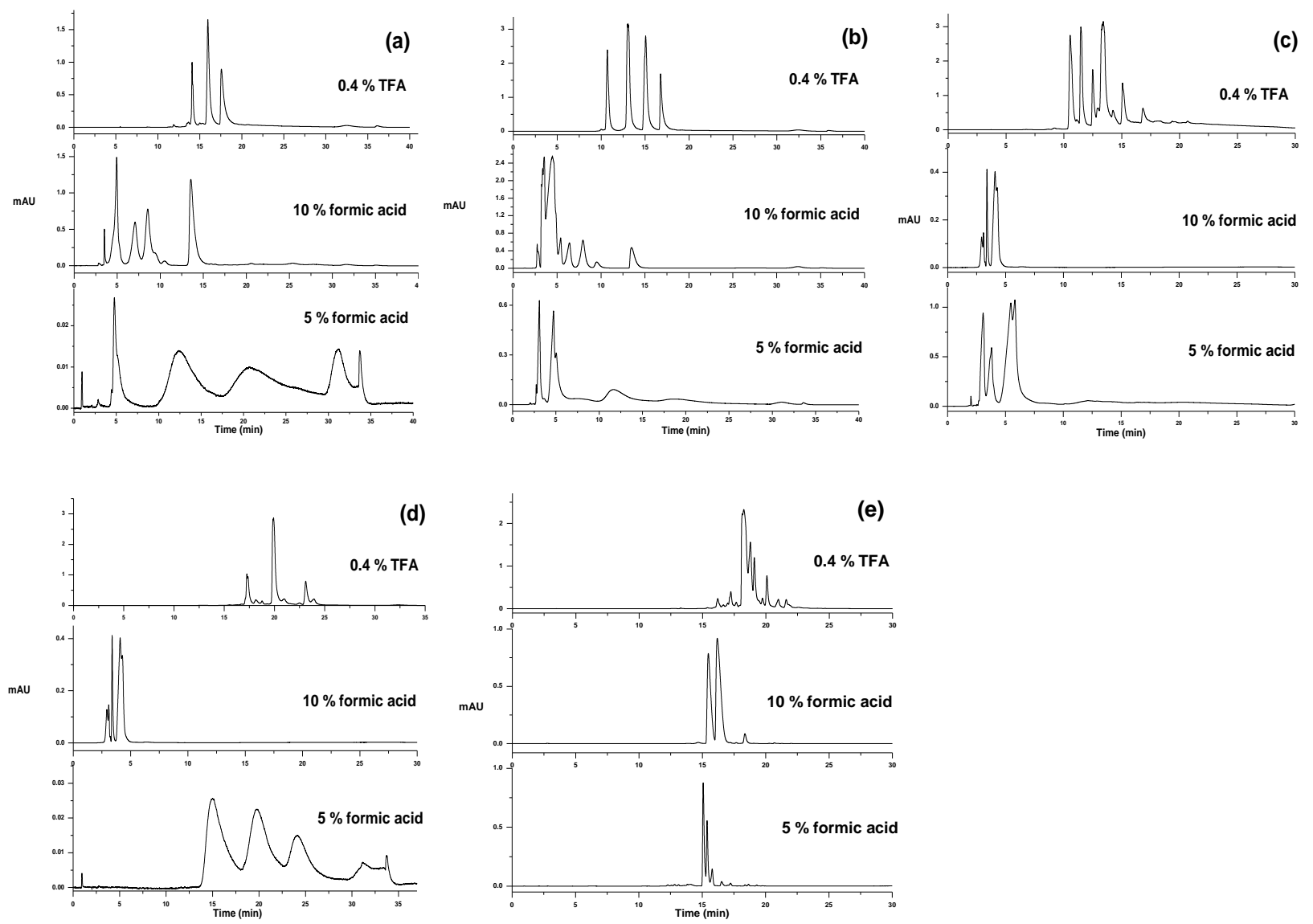

Figure S2: LC- UV chromatograms obtained at $500 \mathrm{~nm}$ for the HILIC analysis of (a) black bean, (b) blueberry, (c) red grape skin, (d) red cabbage and (e) red radish anthocyanins using the scouting gradient (Section 2.3.1) on the BEH Amide column $(150 \mathrm{~mm} \times 1.0 \mathrm{~mm}$ i.d., 1.7 $\mu \mathrm{m})$ with the following mobile phases: $5 \%$ formic acid modified mobile phase at $25{ }^{\circ} \mathrm{C} ; 10$ $\%$ formic acid modified mobile phase at $25{ }^{\circ} \mathrm{C}$; and $0.4 \%$ trifluoroacetic acid modified at 25 ${ }^{\circ} \mathrm{C}$. Injection volumes of $0.5 \mu \mathrm{L}$ were used. For further experimental detail, refer to section 2.3.1. 

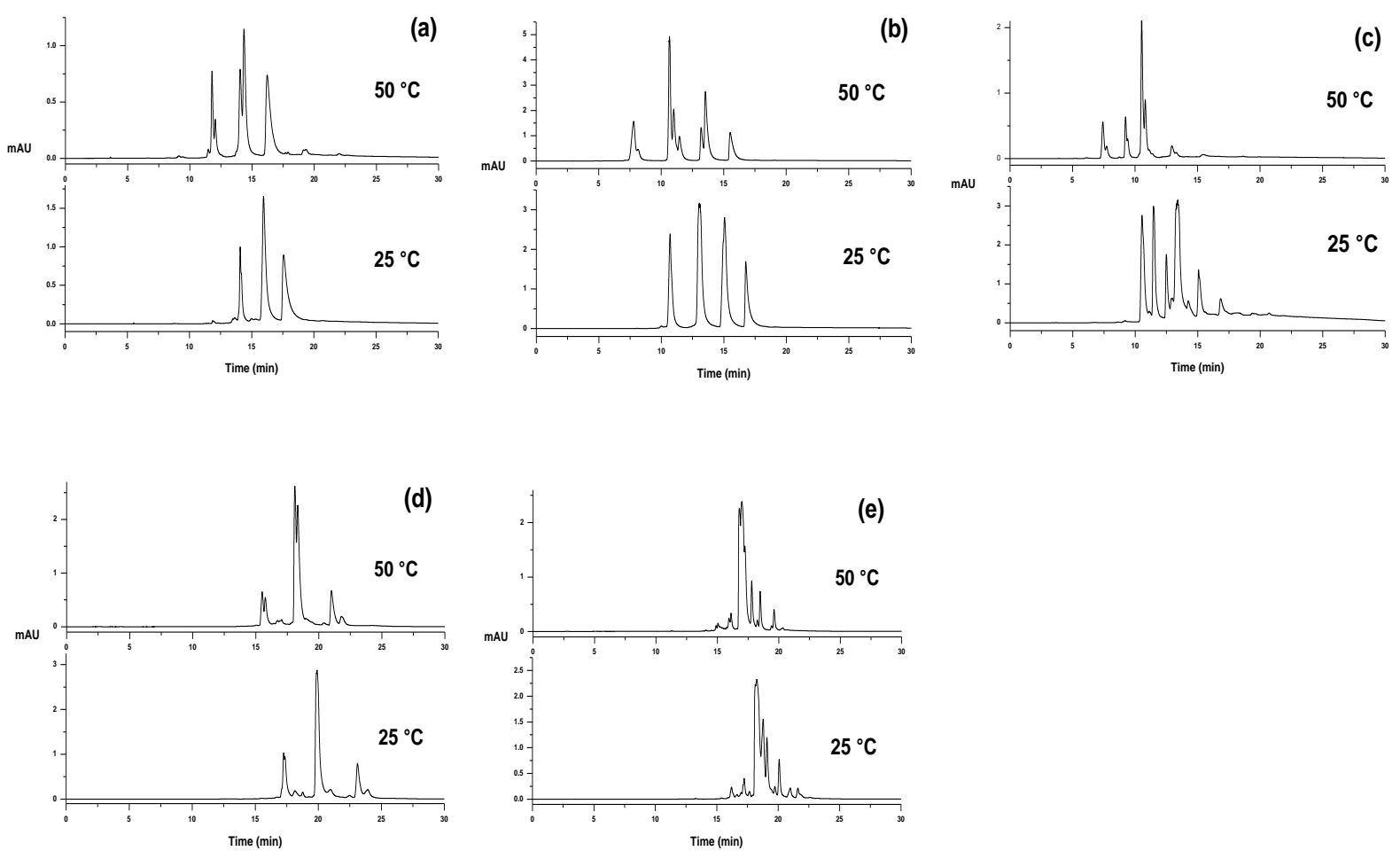

Figure S3: Overview of the effect of temperature on the HILIC separation of (a) black bean, (b) blueberry, (c) red grape skin, (d) red cabbage and (e) red radish anthocyanins. The scouting gradient (Section 2.3.1) was used in all cases with $0.4 \%$ trifluoroacetic acid as mobile phase modifier on the BEH Amide column $(150 \mathrm{~mm} \times 1.0 \mathrm{~mm}$ i.d., $1.7 \mu \mathrm{m})$. Injection volumes of $0.5 \mu \mathrm{L}$ were used. For further experimental detail, refer to section 2.3.1. 


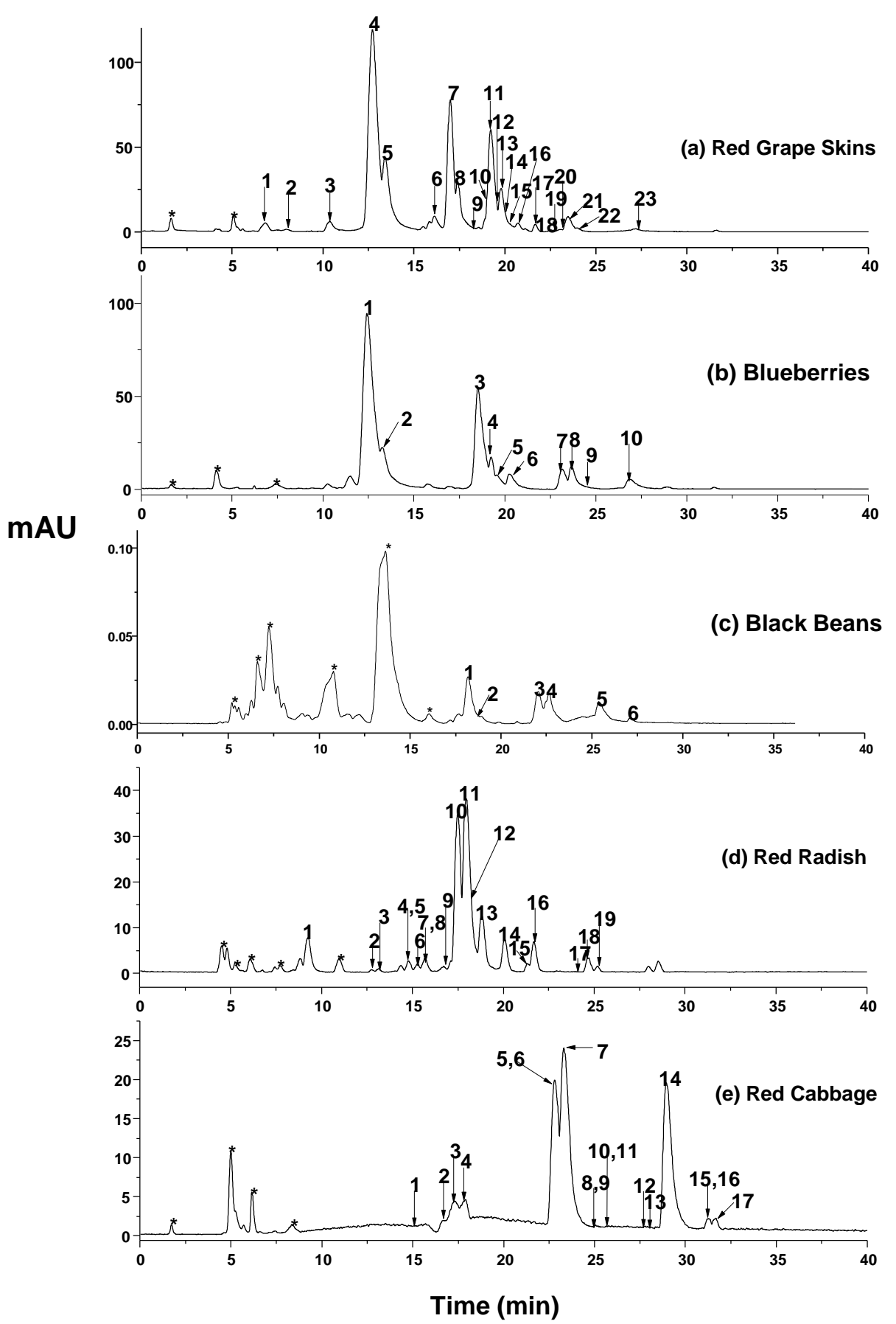

Figure S4: Total ion chromatograms obtained for the HILIC-ESI-MS analysis of anthocyanins in (a) red grape skin, (b) blueberry, (c) black beans, (d) red radish and (e) red cabbage extracts. Analyses were performed at $50{ }^{\circ} \mathrm{C}$ on an amide column $(150 \times 1 \mathrm{~mm}$ id, 1.7 $\mu \mathrm{m} d_{p}$ ) using as mobile phase $0.4 \%$ TFA in acetonitrile (solvent A)/ $0.4 \%$ TFA in water (solvent B). For gradient details and further experimental conditions, refer to Section 2.3.2.* Designates non-anthocyanin compounds. 


\section{Addendum B}

\section{Comprehensive two-dimensional liquid chromatographic analysis of anthocyanins}

C.M. Willemse, M.A. Stander, A.G.J. Tredoux, A. de Villiers, J. Chromatogr. A 1359 (2014) 189-201.

Author contributions: C.M.W. performed all experiments, did initial data analysis and wrote the first draft of the paper. M.A.S., A.G.J.T. and A.d.V. assisted with MS data analysis and edited the manuscript. 


\title{
Comprehensive two-dimensional liquid chromatographic analysis of anthocyanins
}

\author{
Chandré M. Willemse ${ }^{\mathrm{a}}$, Maria A. Stander ${ }^{\mathrm{b}, \mathrm{c}}$, Andreas G.J. Tredoux ${ }^{\mathrm{a}}$, André de Villiers ${ }^{\mathrm{a}, *}$ \\ a Stellenbosch University, Department of Chemistry and Polymer Science, Private Bag X1, Matieland 7602, South Africa \\ b Stellenbosch University, Central Analytical Facility, Private Bag X1, Matieland 7602, South Africa \\ ' Stellenbosch University, Department of Biochemistry, Private Bag X1, Matieland 7602, South Africa
}

\section{A R T I C L E I N F O}

\section{Article history:}

Received 4 April 2014

Received in revised form 25 June 2014

Accepted 16 July 2014

Available online 22 July 2014

\section{Keywords:}

Anthocyanins

Comprehensive two-dimensional liquid

chromatography $(\mathrm{LC} \times \mathrm{LC})$

Hydrophilic interaction chromatography (HILIC)

Reversed phase liquid chromatography (RP-LC)

Phenolic compounds

Orthogonality

\begin{abstract}
A B S T R A C T
Anthocyanins are naturally occurring plant pigments whose accurate analysis is hampered by their complexity and unique chromatographic behaviour associated with on-column conversion reactions. This paper reports the evaluation of off-line comprehensive two-dimensional liquid chromatography $(\mathrm{LC} \times \mathrm{LC})$ for the analysis of anthocyanins. Hydrophilic interaction chromatography (HILIC) was used in the first dimension in combination with reversed phase liquid chromatography (RP-LC) in the second dimension. For the selective detection of anthocyanins, diode array detection was used, while high resolution quadrupole-time-of-flight mass spectrometry (Q-TOF) was used for compound identification. As application, the HILIC $\times$ RP-LC separation of diverse anthocyanins in blueberries, red radish, black beans, red grape skins and red cabbage is demonstrated. Off-line HILIC $\times$ RP-LC revealed information which could not be obtained by one-dimensional HPLC methods, while the structured elution order for the anthocyanins simplifies compound identification and facilitates the comparison of anthocyanin content of natural products by means of contour plots.
\end{abstract}

(C) 2014 Elsevier B.V. All rights reserved.

\section{Introduction}

Anthocyanins are a group of the flavonoid family of phenolic compounds comprising water-soluble pigments that are responsible for the red, blue and violet colours of many flowers, fruits, grains and vegetables [1]. These pigments are important to the food industry and have been recognised for their nutritional value, since they play a role in the reduced risk of a number of chronic and degradative diseases in humans [2,3]. Over the last two decades there has been a significant growth in anthocyanin research due to their potential use in applications varying from food colourants to supplements with potential health benefits [4].

Due to the complexity of many natural products the accurate qualitative and quantitative analysis of anthocyanins remains challenging. The most successful separation of anthocyanins is obtained by reversed phase liquid chromatography (RP-LC) [5]. Coupled to photodiode-array (PDA) detection, selective detection of

\footnotetext{
is Presented at the 13th International Symposium on Hyphenated Techniques in Chromatography and Separation Technology, Bruges, Belgium, 29-31 January 2014.

* Corresponding author. Tel.: +27 21808 3351; fax: +27 218083360.

E-mail address: ajdevill@sun.ac.za (A. de Villiers).
}

anthocyanins is possible [6,7], since they absorb in the visible range around $500-550 \mathrm{~nm}$ in low $\mathrm{pH}$ mobile phase conditions. RP-LC hyphenated with mass spectrometry (MS) or tandem mass spectrometry (MS/MS) provides increased sensitivity and structural elucidation capabilities [8]. Despite these advantages, however, RP-LC-MS also demonstrates some limitations for anthocyanin analysis. First, RP-LC separation of anthocyanins is characterised by extremely low optimal mobile phase velocity, and, when analysed under sub-optimal conditions, very low chromatographic efficiency $[9,10]$. This is a result of inter-conversion between different anthocyanin species in solution, which depends on the $\mathrm{pH}$ and anthocyanin structure [9-11]. Secondly, the chemical diversity and lack of commercially available standards hampers identification, whereas similar fragmentation patterns between different anthocyanin classes complicate structural elucidation. For these reasons, improved chromatographic separation of anthocyanins remains important.

The coupling of two independent LC separations in comprehensive two-dimensional liquid chromatography ( $\mathrm{LC} \times \mathrm{LC})$ offers a powerful approach for the separation of compounds in complex samples [12-15]. In LC $\times$ LC all sample components eluting from the first dimension are also subjected to separation in a second dimension [16], in this manner providing the advantages of increased 
<smiles>[R6]Oc1cc(-c2cc([R2])c(O)c([R2])c2)[o+]c2cc(O)cc([R])c12</smiles>

Dp: $\mathrm{R}_{1}=\mathrm{R}_{2}=\mathrm{OH}, \mathrm{R}_{3}=\mathrm{R}_{4}=\mathrm{H}$

Cy: $\mathrm{R}_{1}=\mathrm{OH}, \mathrm{R}_{2}=\mathrm{R}_{3}=\mathrm{R}_{4}=\mathrm{H}$

Pel: $\mathrm{R}_{1}=\mathrm{R}_{2}=\mathrm{R}_{3}=\mathrm{R}_{4}=\mathrm{H}$

Pt: $\quad \mathrm{R}_{1}=\mathrm{OCH}_{3}, \mathrm{R}_{2}=\mathrm{OH}, \mathrm{R}_{3}=\mathrm{R}_{4}=\mathrm{H}$

Pe: $\mathrm{R}_{1}=\mathrm{OCH}_{3}, \mathrm{R}_{2}=\mathrm{R}_{3}=\mathrm{R}_{4}=\mathrm{H}$

Mv: $\mathrm{R}_{1}=\mathrm{OCH}_{3}, \mathrm{R}_{2}=\mathrm{OCH}_{3}, \mathrm{R}_{3}=\mathrm{R}_{4}=\mathrm{H}$

$$
\mathrm{R}_{3}, \mathrm{R}_{4}=\mathrm{H} \text { or }
$$

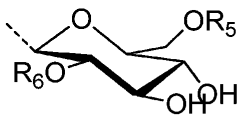

or $\mathrm{R}_{3}=$ arabinoside<smiles>[R5]B=[Bi]C(C)=O</smiles><smiles>CC(=O)/C=C/c1ccc(O)c(O)c1</smiles>
caffeoyl<smiles>CC(=O)/C=C/c1ccc(O)cc1</smiles>
p-coumaroyl<smiles>COc1cc(/C=C/C(C)=O)cc(OC)c1O</smiles><smiles>COc1cc(/C=C/C(C)=O)ccc1O</smiles>

feruloyl<smiles>CCC(=O)CC(=O)O</smiles>

sinapoyl

malonoyl

$$
\mathrm{R}_{6}=\mathrm{R}_{3} \text { or } \mathrm{H}
$$

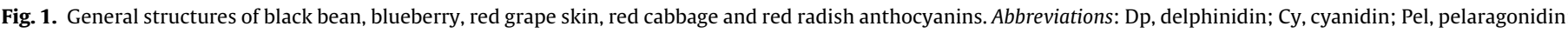
Pt, petunidin; Pe, peonidin; Mv, malvidin.

resolution and selectivity in comparison to one-dimension (1-D) LC.

One of the principal requirements of an efficient $\mathrm{LC} \times \mathrm{LC}$ system is that the separation modes employed be orthogonal. This implies that the separation mechanisms to be used in each dimension be carefully selected in order to minimise the retention correlation between dimensions [17]. Hydrophilic interaction chromatography (HILIC) is receiving increasing attention as an alternative mode to RP-LC for the analysis of polar compounds. In fact, the combination of HILIC and RP-LC provides a high degree of orthogonality, and HILIC $\times$ RP-LC has successfully been applied to the analysis of a range of phenolic compounds [18-21]. However, to the best of our knowledge, LC $\times$ LC has to date not been applied to anthocyanin analysis.

The objective of this study was therefore to develop a HILILC $\times$ RP-LC method, employing the HILIC method recently developed for anthocyanins [22], for the separation of various anthocyanin-rich natural products. As application, several samples characterised by complex and diverse anthocyanin constituents were selected, including blueberries, grape skins, black beans, red cabbage and red radish (Fig. 1).

\section{Experimental}

\subsection{Reagents and materials}

Blueberries, black beans, grapes, red cabbage and red radish were purchased from a local supermarket. HPLC grade acetonitrile, methanol and trifluoroacetic acid (TFA) were purchased from Sigma-Aldrich (Steinheim, Germany) and formic acid from Merck (Darmstadt, Germany). Deionised water was prepared using a MilliQ water purification system (Millipore, Milford, MA, USA). All mobile phases were filtered through $0.45 \mu \mathrm{m}$ HLVP membrane filters (Millipore), and degassed in an ultrasonic bath. The OASIS HLB solid phase extraction (SPE) cartridges $(6 \mathrm{~mL}, 500 \mathrm{mg})$ were from Waters (Milford, MA, USA).

\subsection{Sample preparation}

Each of the five samples were extracted in the same manner as reported previously [22]. The extracts were loaded on a preconditioned SPE cartridge, rinsed with acidified water and eluted with $3 \mathrm{~mL} \times 1 \mathrm{~mL}$ methanol/formic acid $(95 / 5,(v / v))$ [22]. The methanolic fraction containing the anthocyanins was evaporated to dryness and re-dissolved in $0.5 \mathrm{~mL}$ methanol/formic acid $(95 / 5,(v / v))$ prior to HPLC analysis. No loss of anthocyanins was observed following SPE clean-up.

\subsection{Instrumentation}

One-dimensional HILIC-UV and RP-LC-UV analyses as well as off-line HILIC $\times$ RP-LC analyses were performed on a Waters Acquity UPLC system equipped with a binary pump, sample manager, column oven compartment, photodiode array (PDA) detector ( $500 \mathrm{~nL}$ flow cell, $10 \mathrm{~mm}$ path length) and controlled by Waters Empower software (Waters, Milford, MA, USA). UV-vis chromatograms were recorded at $500 \mathrm{~nm}$ using an acquisition rate of $10 \mathrm{~Hz}$.

LC-MS and LC-MS ${ }^{\mathrm{E}}$ analyses were performed on a Waters Acquity UPLC system equipped with a binary solvent manager, sample manager and column oven, and interfaced through an electrospray ionisation (ESI) source to a Waters Synapt G2 quadrupole time-of-flight (Q-TOF) mass spectrometer.

\subsection{Chromatographic conditions}

\subsubsection{Off-line HILIC $\times R P$-LC analyses}

HILIC separation was performed on an XBridge BEH Amide column $(150 \mathrm{~mm} \times 4.6 \mathrm{~mm}$ i.d., $2.5 \mu \mathrm{m}$ particles, Waters $)$ with a 
Table 1

HILIC gradient details for each of the samples analysed.

\begin{tabular}{|c|c|c|}
\hline Sample & Gradient & Injection volume $(\mu \mathrm{L})$ \\
\hline Blueberries & $4-35 \%$ B (0-92.58 min), 35-100\% B (92.58-95.22 min), 100\% B for $5 \mathrm{~min}$ & 5 \\
\hline Grape skins & $4-35 \%$ B $(0-92.58 \mathrm{~min}), 35-100 \%$ B $(92.58-95.22 \mathrm{~min}), 100 \%$ B for $5 \mathrm{~min}$ & 6 \\
\hline Red cabbage & $13-40 \%$ B (0-105.80 min $), 40-100 \%$ B (105.80-108.45 min $), 100 \%$ B for 5 min & 10 \\
\hline Red radish & $15-33.5 \%$ B (0-92.58 min), 33.5-100\% B (92.58-97.87 min), $100 \%$ B for 5 min & 10 \\
\hline Black beans & $5-30 \%$ B $(0-71.42 \mathrm{~min}), 30-100 \%$ B $(71.42-74.06 \mathrm{~min}), 100 \%$ B for $5 \mathrm{~min}$ & 10 \\
\hline
\end{tabular}

Krudcatcher UHPLC pre-column filter (Phenomenex, Torrace USA). The binary mobile phase consisted of (A) 0.4\% TFA in acetonitrile and (B) $0.4 \%$ TFA in water. Four different gradients were used, which are tabulated together with the relevant injection volumes in Table 1. Injections were performed in the 'partial loop with needle overfill' mode using acetonitrile/TFA $(99.6 / 0.4, v / v)$ as the weak needle wash solvent. The flow rate was $200 \mu \mathrm{L} / \mathrm{min}$, and all analyses were performed at $50^{\circ} \mathrm{C}$.

For off-line HILIC $\times$ RP-LC analyses half minute fractions $(0.1 \mathrm{~mL}$ each) of the HILIC effluent were collected using a BIO-RAD 2110 fraction collector (Corston, UK). Collected fractions were transferred to $1.5 \mathrm{~mL}$ vials containing $250 \mu \mathrm{L}$ glass inserts and were kept under nitrogen prior to RP-LC analyses.

RP-LC separation was performed in the second dimension on a Kinetex C18 column $(50 \mathrm{~mm} \times 4.6 \mathrm{~mm}$ i.d., $2.6 \mu \mathrm{m}$ particles $)$ with a security guard column (Phenomenex, for $4.6 \mathrm{~mm}$ i.d. columns). The binary mobile phases consisted of $7.5 \%(v / v)$ formic acid in water $(\mathrm{A})$ and $7.5 \%(\mathrm{v} / \mathrm{v})$ formic acid in acetonitrile (B). The gradient used was as follows: $1 \% \mathrm{~B}(0-0.75 \mathrm{~min}), 1-13.5 \% \mathrm{~B}(0.75-8.50 \mathrm{~min})$, 13.5-23.5\% B (8.50-16.75 min), 23.5-28.5\% B (16.75-19.5 min), $28.5 \%$ B isocratic (19.5-24.5 min), before returning to initial conditions at $25.0 \mathrm{~min}$. The flow rate was $500 \mu \mathrm{L} / \mathrm{min}$ and the column was re-equilibrated for $15 \mathrm{~min}$ before the next analysis. The column temperature was set to $50^{\circ} \mathrm{C}$. An injection volume of $2 \mu \mathrm{L}$ in the 'partial loop with needle overfill' mode was used, with $7.5 \%$ formic acid as the weak needle wash solvent.

\subsubsection{HPLC-ESI-MS analyses}

LC-Q-TOF-MS data were acquired in positive ionisation mode with a scan range of 200-2000 amu. MS ${ }^{\mathrm{E}}$ (an acquisition mode performed concurrently with full scan acquisition in a single analysis, where fragmentation is performed using a collision energy ramp) data were acquired across a mass range of 40-2000 amu. All instrumental parameters were the same as previously reported [22]. Data were collected and acquired using MassLynx v.4.1 software (Waters).

\subsection{Data handling}

Raw UV-vis data were exported into OriginPro 8.5 software (OriginLab Corporation, USA) to construct the figures. Excel (Microsoft Corporation, USA) and Matlab (Mathworks Inc., Natick, Massachusetts, USA) were used to calculate orthogonality data according to Ref. [23]. For the calculation of surface coverage, experimental retention data obtained for each sample were normalised in both dimensions [23].

\section{Results and discussion}

\subsection{Method optimisation: choice of experimental conditions}

RP-LC was selected as separation mode in the second dimension of LC $\times$ LC, due to its high efficiency, speed and compatibility with MS detection. The RP-LC method used here was based on the optimal conditions reported for anthocyanin analysis by de Villiers et al. $[9,10]$. In short, a highly acidic mobile phase (7.5\% formic acid) was used at a relatively low flow rate $(500 \mu \mathrm{L} / \mathrm{min}$ on a $4.6 \mathrm{~mm}$ i.d. column ) and elevated temperature $\left(50^{\circ} \mathrm{C}\right)$. Under these conditions, the detrimental effect of on-column inter-conversion reactions on the chromatographic efficiency of anthocyanins is largely negated [9]. Highly acidic mobile phases also ensure that the anthocyanins are predominantly in their flavylium cationic form, which absorb maximally in the visible region around $500-550 \mathrm{~nm}$ and therefore allows group selective detection at these wavelengths [7]. A superficially porous $\mathrm{C} 18$ column (Kinetex, $50 \mathrm{~mm} \times 4.6 \mathrm{~mm}, 2.6 \mu \mathrm{m}$ $d_{p}$ ) was used, as this column has been shown to provide good chromatographic performance for the analysis of various phenolic compounds [24]. Following optimisation of the gradient profile, the Kinetex column provided good separation of the diverse anthocyanins present in all the samples for gradient times of $30 \mathrm{~min}$ (including re-equilibration) (Fig. S1).

The most important consideration for the first dimension separation was to obtain a high degree of orthogonality relative to the RP-LC separation used in the second dimension. HILIC and RPLC have been shown to be a versatile, orthogonal combination for the LC $\times$ LC separation of diverse phenolic compounds [17-20]. The HILIC method recently reported for the separtion of anthocyanins was therefore used in the first dimension [22], with some modification with regard to the column i.d. and gradient time. A wide-bore ( $150 \mathrm{~mm} \times 4.6 \mathrm{~mm}$ i.d.) column was selected since anthocyanins show very low optimal flow rates in HILIC [22] - on the $4.6 \mathrm{~mm}$ column, the optimal flow rate is only $0.2 \mathrm{~mL} / \mathrm{min}$. This flow is ideal for off-line LC $\times$ LC when short sampling times are used. The use of a narrow-bore $1 \mathrm{~mm}$ column, for example, would result in an optimal flow rate of $\sim 6 \mu \mathrm{L} / \mathrm{min}$ [22], which is below the capabilities of most commercial HPLC instruments and unpractical from a fraction collection point of view. Furthermore, $4.6 \mathrm{~mm}$ i.d. columns provide higher volume loadability and peak capacity (the latter due to the diminished effect of extra-column band broadening) than narrowbore columns commonly used in LC $\times$ LC. As can be seen in Fig. S2, which summerises 1-D HILIC analyses of the samples, good chromatographic peak shapes were obtained for the anthocyanins in each of these samples.

Hyphenation of two separations in $\mathrm{LC} \times \mathrm{LC}$ can be performed in three ways, i.e. on-line, off-line and stop-flow, with the former two being most frequently used. In the on-line approach, fractions from the first dimension are continuously transferred to the second dimension column by employing a switching valve and fast second dimension separations, whereas in stop-flow mode the flow in the first dimension is stopped during the second dimension analysis of the transferred fraction [18]. Off-line LC $\times$ LC involves the collection of fractions from the first dimension which are then reinjected onto the second dimension column. This approach places less restrictions on the second dimension analysis time, and by allowing maximum exploitation of each of the 1-D separations [25] provides the highest peak capacities, typically in the order of a few thousand [18-20].

Off-line HILIC $\times$ RP-LC was selected in the current study for several reasons. First, since off-line coupling places less restriction on the second dimension analysis time, this mode provides higher overall resolution and higher peak capacities, although at the cost of longer analysis times, compared to on-line $\operatorname{LC} \times \operatorname{LC}[18,20,25,26]$. This is due to separations in the two dimensions being carried out independently; therefore second dimension analyses can be 
performed under optimal conditions (low flow rate, longer gradient times) for maximum resolution. Secondly, in the case of anthocyanins as target analytes, the very fast second dimension analyses required for on-line operation will result in much worse performances than for 'normal' analytes. This is because band-broadening associated with the hydration secondary equilibrium is especially detrimental for short analysis times $[9,10]$. Compared to stop-flow operation, the off-line approach offers a simpler experimental configuration at the cost of automation $[25,27]$.

The coupling of HILIC and RP-LC in LC $\times$ LC is challenging due to the relative elution strengths of the solvents in the two dimensions $[14,28,29]$. Injection of large volumes of HILIC fractions onto the RP-LC column results in severe injection band broadening which impacts negatively on the second dimension separation [19,29]. On the $50 \mathrm{~mm} \times 4.6 \mathrm{~mm}$ i.d. C18 column used in the second dimension, maximally $2 \mu \mathrm{L}$ of the HILIC fractions could be injected to avoid peak distortion of especially early eluting compounds.

Since complete fraction transfer is not possible in off-line LC $\times$ LC, this implies that some sensitivity loss will occur. Exactly how much depends on the sampling time, ${ }^{1} t_{\mathrm{s}}$, the first dimension volumetric flow rate and the maximum permissible injection volume in the second dimension. A sampling time of $30 \mathrm{~s}$ was selected in the current study since this provided an acceptable degree of under-sampling of the first dimension peaks (see Table 3 further). (Note that shorter sampling times would decrease under-sampling, but significantly increase the total analysis time $[25,27,30])$. Using a first dimension flow rate of $0.2 \mathrm{~mL} / \mathrm{min}$, fraction volumes of $100 \mu \mathrm{L}$ were obtained, which are practical to work with in off-line separations. Therefore, $\sim 2 \%(2 \mu \mathrm{L})$ of each HILIC fraction is injected onto the second dimension column. This loss in sensitivity is partially compensated for by employing relatively large injection volumes, enabled by the use of a $4.6 \mathrm{~mm}$ i.d. column in the first dimension. Furthermore, injecting highly concentrated extracts permits the detection of even low-level anthocyanins under these conditions (see further).

\subsection{HILIC $\times R P-L C$ analysis of anthocyanins in natural products}

Contour plots obtained for the off-line HILIC $\times$ RP-LC analyses of blueberry, grape skin, black bean, red cabbage and red radish anthocyanins are shown in Figs. 2-5. Identification of peaks in these contour plots was based on correlation of retention times with both 1-dimensional HILIC-ESI-MS(/MS) and RP-LC-ESI-MS(/MS) data. MS detection was performed in positive ionisation mode and compounds were identified based on accurate mass data as well as fragmentation information obtained from $\mathrm{MS}^{\mathrm{E}}$ and $\mathrm{MS} / \mathrm{MS}$ experiments and comparison with previous literature reports [8,31-38]. A summary of the MS data for each of the identified compounds is presented in Table 2. For further details on the identification of anthocyanins in each of the investigated samples, the reader is referred to Ref. [22].

\subsubsection{Blueberries and black beans}

Blueberries and black beans contained relatively simple anthocyanins, mainly comprising anthocyanidin-monoglycosides. For blueberries, 15 anthocyanins were identified (Fig. 2a). Anthocyanidin-glucosides and anthocyanidingalactosides were not resolved in HILIC, whereas in RP-LC the anthocyanidin-galactosides elute before the corresponding anthocyanidin-glucosides [22]. Anthocyanidin-arabinosides elute last in RP-LC, and much earlier than the corresponding-glucosides and-galactosides in HILIC. As a result, malvidin-3-glucoside (3) and malvidin-3-galactoside (4), peonidin-3-glucoside (5) and peonidin-3-galactoside (7), petunidin-3-glucoside (9) and petunidin-3-galactoside (10), cyanidin-3-glucoside (11) and cyanidin-3-galactoside (13) as well as delphinidin-3-glucoside
(14) and delphinidin-3-galactoside (15) co-elute in HILIC, but are separated in RP-LC based on the glycosidic moiety. On the other hand, cyanidin-3-galactoside (11) and delphinidin-3-arabinoside (12) co-elute in HILIC due to the similar polarity of these compounds despite differences in terms of both the anthocyanidin base and glycosidic groups (the same is true for compounds $\mathbf{4}, 5$ and $\mathbf{6}$ ).

Compounds which co-eluted in RPLC, for example cyanidin-3arabinoside (8) and petunidin-3-glucoside (9) were separated in HILIC primarily based on the sugar moiety. However, several coeluting compounds were not separated in either of the dimensions, such as peonidin-3-galactoside $(\mathbf{5})$ and petunidin-3-arabinoside $(\mathbf{6})$ as well as delphinidin-3-arabinoside (12) and cyanidin-3-glucoside (13).

Ten anthocyanins were identified in black beans (Fig. 2b). RPLC provided complete separation of all anthocyanins, whereas the HILIC dimension added little relevant information for this sample. Interestingly though, three distinct groups of compounds are observed in the HILIC dimension. The first of these includes the malvidin and peonidin derivatives, the second more strongly retained (in HILIC) group includes the petunidin and cyanidin derivatives, while the most retained group contains the delphinidin derivatives. The only acylated compound, delphinidin-3-acetylglucoside (4), does not follow this trend, as acylation results in earlier elution in HILIC. Similar to blueberries, all anthocyanidin-galactosides and -glucosides co-elute in HILIC but were resolved in RP-LC.

\subsubsection{Red radish}

Red radish anthocyanins comprise a mixture of monoor di-acylated derivatives of anthocyanidin-di-(or-tri)glucosides. The major anthocyanidin present in red radish is pelaragonidin, with the exception of two compounds, cyanidin-3(glycosyl)rhamnoside (1) and cyanidin-3-(diglycosyl)rhamnoside (8) [22,32]. A total of 21 anthocyanins spanning a wide range of concentrations were identified (Fig. 3). Red radish anthocyanins were relatively strongly retained on the amide column due to their high degree of glycosylation, and also generally displayed high RP-LC retention due to their high degree of acylation. Unlike blueberries and black beans, no clear elution pattern according to common structural characteristics was evident for red radish anthocyanins. This is due to the structural diversity of these compounds, and the contrasting effects of the nature, number and position of acyl groups on retention in both dimensions.

For example, pelaragonidin-3-( $p$-coumaroyl)diglucoside5-(malonoyl)glucoside $\left(\mathbf{6}^{\mathbf{C}}\right)$ and pelaragonidin-3-(feruloyl)diglucoside-5-glucoside $\left(\mathbf{7}^{\mathbf{B}}\right)$ were only partially separated in HILIC, but were well resolved in RP-LC due to more efficient differentiation according to the degree of acylation in the latter mode. On the other hand, pelaragonidin-3-diglucoside-5-glucoside (14) and pelaragonidin-3-(feruloyl)diglucoside-5-glucoside $\left(\mathbf{7}^{\mathbf{A}}\right)$ as well as pelaragonidin-3-(feruloyl)diglucoside-5(malonoyl)glucoside $\left(\mathbf{5}^{\mathbf{D}}\right)$ and pelaragonidin-3-(caffeoyl)diglucoside-5(malonoyl)glucoside (10) co-eluted in RP-LC, since the separation of highly glycosylated species with the same degree of acylation is not very efficient according to the nature of the acyl-group. These compounds were however separated in HILIC as a result of the polarity of acyl-substituents. While acylation generally results in a decrease in retention in HILIC, caffeoylated derivatives show the highest retention among the acylated derivatives, resulting in retention times for these compounds which are close to those of the corresponding non-acylated anthocyanins. This allows clear separation of compounds $\mathbf{5}^{\mathbf{D}}$ and $\mathbf{1 0}$ in HILIC.

Several isomeric compounds were identified in red radish (compounds labelled $\mathbf{5}^{\mathbf{A}-\mathbf{D}}, \mathbf{6}^{\mathbf{A}-\mathbf{C}}$ and $\mathbf{7}^{\mathbf{A}, \mathbf{B}}$ in Table 2). These compounds have identical molecular formulae and fragmentation patterns, but are clearly separated by HILIC and/or RP-LC. Most likely these 
Table 2

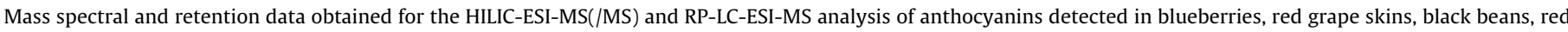
cabbage and red radish under optimised gradient conditions for each sample (refer to Section 2.4.1 for details).

\begin{tabular}{|c|c|c|c|c|c|c|c|}
\hline Compound number ${ }^{\mathrm{a}}$ & HILIC $t_{R}^{\mathrm{b}}(\mathrm{min})$ & $\begin{array}{l}\mathrm{RP}-\mathrm{LC} t_{R}^{\mathrm{b}} \\
(\mathrm{min})\end{array}$ & Identity & $\begin{array}{l}\text { Molecular } \\
\text { formula }[\mathrm{M}]^{+}\end{array}$ & $\begin{array}{l}\text { Experimental } \\
\text { mass }(m / z)\end{array}$ & $\begin{array}{l}\mathrm{MS}^{\mathrm{E}} \\
\text { Fragments }\end{array}$ & References \\
\hline \multicolumn{8}{|l|}{ Blueberries } \\
\hline 1 & 23.91 & 9.34 & Malvidin-3-arabinoside & $\mathrm{C}_{22} \mathrm{H}_{23} \mathrm{O}_{11}$ & 463.1135 & $\begin{array}{l}331^{\mathrm{c}}, 315 \\
287,242\end{array}$ & {$[8,22,32]$} \\
\hline 2 & 24.79 & 8.63 & Peonidin-3-arabinoside & $\mathrm{C}_{21} \mathrm{H}_{21} \mathrm{O}_{10}$ & 433.1244 & $301^{\mathrm{c}}$ & {$[8,22,32]$} \\
\hline 3 & 32.31 & 8.35 & Malvidin-3-galactoside & $\mathrm{C}_{23} \mathrm{H}_{25} \mathrm{O}_{12}$ & 493.1346 & $\begin{array}{l}331^{\mathrm{c}}, 315 \\
242\end{array}$ & {$[8,22,32,39]$} \\
\hline 4 & 33.44 & 8.83 & Malvidin-3-glucoside & $\mathrm{C}_{23} \mathrm{H}_{25} \mathrm{O}_{12}$ & 493.1343 & $\begin{array}{l}331^{\mathrm{c}}, 315 \\
287,242\end{array}$ & {$[8,22,32,39]$} \\
\hline 5 & 33.75 & 8.17 & Peonidin-3-galactoside & $\mathrm{C}_{22} \mathrm{H}_{23} \mathrm{O}_{11}$ & 463.1240 & $\begin{array}{l}301^{\mathrm{c}}, 286 \\
258,149\end{array}$ & {$[8,22,32,39]$} \\
\hline 6 & 33.99 & 7.73 & Petunidin-3-arabinoside & $\mathrm{C}_{21} \mathrm{H}_{21} \mathrm{O}_{11}$ & 449.1091 & $317^{c}, 287$ & {$[8,22,32]$} \\
\hline 7 & 34.58 & 8.19 & Peonidin-3-glucoside & $\mathrm{C}_{22} \mathrm{H}_{23} \mathrm{O}_{11}$ & 463.1240 & $\begin{array}{l}301^{\mathrm{c}}, 286 \\
258,149\end{array}$ & {$[8,22,32,39]$} \\
\hline 8 & 35.07 & 6.88 & Cyanidin-3-arabinoside & $\mathrm{C}_{20} \mathrm{H}_{19} \mathrm{O}_{10}$ & 419.0979 & $\begin{array}{l}287^{c}, 213 \\
149\end{array}$ & {$[8,22,32,39]$} \\
\hline 9 & 42.19 & 7.22 & Petunidin-3-galactoside & $\mathrm{C}_{22} \mathrm{H}_{23} \mathrm{O}_{12}$ & 479.1197 & $\begin{array}{l}317^{c}, 302 \\
274,149\end{array}$ & {$[8,22,32,39]$} \\
\hline 10 & 42.90 & 7.27 & Petunidin-3-glucoside & $\mathrm{C}_{22} \mathrm{H}_{23} \mathrm{O}_{12}$ & 479.1197 & $\begin{array}{l}317^{c}, 302 \\
274,149\end{array}$ & {$[8,22,32,39]$} \\
\hline 11 & 43.06 & 5.92 & Cyanidin-3-galactoside & $\mathrm{C}_{21} \mathrm{H}_{21} \mathrm{O}_{11}$ & 449.1080 & $287^{c}, 213$ & {$[8,22,32,39]$} \\
\hline 12 & 43.76 & 5.97 & Delphinidin-3-arabinoside & $\mathrm{C}_{20} \mathrm{H}_{19} \mathrm{O}_{11}$ & 435.0925 & $303^{c}$ & {$[8,22,32]$} \\
\hline 13 & 44.51 & 5.97 & Cyanidin-3-glucoside & $\mathrm{C}_{21} \mathrm{H}_{21} \mathrm{O}_{11}$ & 449.1080 & $287^{c}, 213$ & {$[8,22,32,39]$} \\
\hline 14 & 52.60 & 5.02 & Delphinidin-3-galactoside & $\mathrm{C}_{21} \mathrm{H}_{21} \mathrm{O}_{12}$ & 465.1039 & $303^{c}$ & {$[8,22,32,39]$} \\
\hline 15 & 53.31 & 5.47 & Delphinidin-3-glucoside & $\mathrm{C}_{21} \mathrm{H}_{21} \mathrm{O}_{12}$ & 465.1040 & $303^{c}, 149$ & {$[8,22,32,39]$} \\
\hline \multicolumn{8}{|l|}{ Black beans } \\
\hline 1 & 30.00 & 8.82 & Malvidin-3-galactoside & $\mathrm{C}_{23} \mathrm{H}_{25} \mathrm{O}_{12}$ & 493.1343 & $\begin{array}{l}331^{\mathrm{c}}, 315 \\
287,242\end{array}$ & {$[8,22]$} \\
\hline 2 & 30.89 & 8.39 & Malvidin-3-glucoside & $\mathrm{C}_{23} \mathrm{H}_{25} \mathrm{O}_{12}$ & 493.1343 & $\begin{array}{l}331^{\mathrm{c}}, 315 \\
287,242\end{array}$ & {$[8,22]$} \\
\hline 3 & 31.89 & 8.06 & Peonidin-3-glucoside & $\mathrm{C}_{22} \mathrm{H}_{23} \mathrm{O}_{11}$ & 463.1235 & $301^{c}$ & {$[8,22]$} \\
\hline 4 & 32.05 & 9.28 & Delphinidin-3-acetylglucoside & $\mathrm{C}_{23} \mathrm{H}_{23} \mathrm{O}_{13}$ & 507.1139 & $303.05^{c}$ & {$[8,22]$} \\
\hline 5 & 39.81 & 7.03 & Petunidin-3-galactoside & $\mathrm{C}_{22} \mathrm{H}_{23} \mathrm{O}_{12}$ & 479.1184 & $\begin{array}{l}317^{c}, 302 \\
274,242\end{array}$ & {$[8,22]$} \\
\hline 6 & 40.44 & 7.10 & Petunidin-3-glucoside & $\mathrm{C}_{22} \mathrm{H}_{23} \mathrm{O}_{12}$ & 479.1189 & $317^{c}, 302$ & {$[8,22]$} \\
\hline 7 & 41.38 & 5.84 & Cyanidin-3-galactoside & $\mathrm{C}_{21} \mathrm{H}_{21} \mathrm{O}_{11}$ & 449.1102 & $287^{c}$ & {$[8,22]$} \\
\hline 8 & 41.85 & 6.34 & Cyanidin-3-glucoside & $\mathrm{C}_{21} \mathrm{H}_{21} \mathrm{O}_{11}$ & 449.1085 & $287^{c}, 213$ & {$[8,22]$} \\
\hline 9 & 50.24 & 4.98 & Delphinidin-3-galactoside & $\mathrm{C}_{21} \mathrm{H}_{21} \mathrm{O}_{12}$ & 465.1044 & $303^{c}$ & {$[8,22]$} \\
\hline 10 & 50.62 & 5.42 & Delphinidin-3-glucoside & $\mathrm{C}_{21} \mathrm{H}_{21} \mathrm{O}_{12}$ & 465.1035 & $303^{c}$ & {$[8,22]$} \\
\hline \multicolumn{8}{|l|}{ Red radish } \\
\hline 1 & 25.57 & 10.14 & Cyanidin-3-(glycosyl) rhamnoside & $\mathrm{C}_{27} \mathrm{H}_{31} \mathrm{O}_{15}$ & 595.1678 & $433,287^{c}$ & {$[22,32]$} \\
\hline 2 & 26.32 & 15.24 & $\begin{array}{l}\text { Pelaragonin-3-(feruloyl) } \\
\text { (feruloyl)diglucoside-5- } \\
\text { (malonoyl)glucoside }\end{array}$ & $\mathrm{C}_{56} \mathrm{H}_{59} \mathrm{O}_{29}$ & 1195.3137 & $\begin{array}{l}519,271^{\mathrm{c}} \\
177,149\end{array}$ & {$[22,32]$} \\
\hline 3 & 26.45 & 14.86 & $\begin{array}{l}\text { Pelaragonin-3- } \\
\text { ( } p \text {-coumaroyl) } \\
\text { (feruloyl)diglucoside-5- } \\
\text { (malonoyl)glucoside }\end{array}$ & $\mathrm{C}_{55} \mathrm{H}_{57} \mathrm{O}_{28}$ & 1165.3037 & $\begin{array}{l}917,519, \\
271^{\mathrm{c}}\end{array}$ & {$[22,32]$} \\
\hline 4 & 28.33 & 6.98 & Pelaragonin-3-glucoside-5-glucoside & $\mathrm{C}_{27} \mathrm{H}_{31} \mathrm{O}_{15}$ & 595.1687 & $271^{\mathrm{c}}$ & {$[22,32]$} \\
\hline $5^{\mathrm{Ad}}$ & 28.49 & 13.77 & $\begin{array}{l}\text { Pelaragonin-3-(feruloyl) } \\
\text { diglucoside-5-(malonoyl) } \\
\text { glucoside }\end{array}$ & $\mathrm{C}_{46} \mathrm{H}_{51} \mathrm{O}_{26}$ & 1019.2676 & $\begin{array}{l}771,519, \\
271^{\mathrm{c}}\end{array}$ & {$[22,32]$} \\
\hline $6^{\mathrm{A}}$ & 31.60 & 13.19 & $\begin{array}{l}\text { Pelaragonin-3- } \\
\text { ( } p \text {-coumaroyl)diglucoside-5- } \\
\text { (malonoyl)glucoside }\end{array}$ & $\mathrm{C}_{45} \mathrm{H}_{49} \mathrm{O}_{25}$ & 989.2566 & $\begin{array}{l}741,519, \\
271^{\mathrm{c}}\end{array}$ & {$[22,32]$} \\
\hline $7^{A}$ & 32.71 & 12.82 & $\begin{array}{l}\text { Pelaragonin-3-(feruloyl) } \\
\text { diglucoside-5-glucoside }\end{array}$ & $\mathrm{C}_{43} \mathrm{H}_{49} \mathrm{O}_{23}$ & 933.2651 & $\begin{array}{l}933,771 \\
433,271^{c}\end{array}$ & {$[22,32]$} \\
\hline $5^{\mathrm{B}}$ & 33.64 & 11.50 & $\begin{array}{l}\text { Pelaragonin-3-(feruloyl) } \\
\text { diglucoside-5-(malonoyl) } \\
\text { glucoside }\end{array}$ & $\mathrm{C}_{46} \mathrm{H}_{51} \mathrm{O}_{26}$ & 1019.2663 & $519,271^{\mathrm{c}}$ & {$[22,32]$} \\
\hline 8 & 34.47 & 9.70 & Cyanidin-3-(diglucosyl)rhamnoside & $\mathrm{C}_{33} \mathrm{H}_{41} \mathrm{O}_{20}$ & 757.2194 & $287^{c}$ & {$[22,32]$} \\
\hline $6^{\mathrm{B}}$ & 35.14 & 11.51 & $\begin{array}{l}\text { Pelaragonin-3- } \\
\text { (p-coumaroyl)diglucoside-5- } \\
\text { (malonoyl)glucoside }\end{array}$ & $\mathrm{C}_{45} \mathrm{H}_{49} \mathrm{O}_{25}$ & 989.2567 & $\begin{array}{l}741,519, \\
271^{\mathrm{c}}\end{array}$ & {$[22,32]$} \\
\hline $5^{\mathrm{C}}$ & 36.39 & 13.53 & $\begin{array}{l}\text { Pelaragonin-3-(feruloyl) } \\
\text { diglucoside-5-(malonoyl) } \\
\text { glucoside }\end{array}$ & $\mathrm{C}_{46} \mathrm{H}_{51} \mathrm{O}_{26}$ & 1019.2662 & $\begin{array}{l}771,519 \\
271^{\mathrm{c}}, 149\end{array}$ & {$[22,32]$} \\
\hline $6^{C}$ & 37.48 & 13.05 & $\begin{array}{l}\text { Pelaragonin-3- } \\
\text { ( } p \text {-coumaroyl)diglucoside-5- } \\
\text { (malonoyl)glucoside }\end{array}$ & $\mathrm{C}_{45} \mathrm{H}_{49} \mathrm{O}_{25}$ & 989.2564 & $\begin{array}{l}741,519 \\
271^{\mathrm{c}}\end{array}$ & {$[22,32]$} \\
\hline $5^{\mathrm{D}}$ & 37.71 & 11.26 & $\begin{array}{l}\text { Pelaragonin-3-(feruloyl) } \\
\text { diglucoside-5-(malonoyl) } \\
\text { glucoside }\end{array}$ & $\mathrm{C}_{46} \mathrm{H}_{51} \mathrm{O}_{26}$ & 1019.2662 & $\begin{array}{l}771,519 \\
271^{\mathrm{c}}, 149\end{array}$ & {$[22,32]$} \\
\hline
\end{tabular}


Table 2 (Continued)

\begin{tabular}{|c|c|c|c|c|c|c|c|}
\hline Compound number ${ }^{\mathrm{a}}$ & HILIC $t_{R}^{\mathrm{b}}(\mathrm{min})$ & $\begin{array}{l}\mathrm{RP}-\mathrm{LC} t_{R}^{\mathrm{b}} \\
(\mathrm{min})\end{array}$ & Identity & $\begin{array}{l}\text { Molecular } \\
\text { formula }[\mathrm{M}]^{+}\end{array}$ & $\begin{array}{l}\text { Experimental } \\
\text { mass }(m / z)\end{array}$ & $\begin{array}{l}\mathrm{MS}^{\mathrm{E}} \\
\text { Fragments }\end{array}$ & References \\
\hline $7^{\mathrm{B}}$ & 38.11 & 12.47 & $\begin{array}{l}\text { Pelaragonin-3-(feruloyl) } \\
\text { diglucoside-5-glucoside }\end{array}$ & $\mathrm{C}_{43} \mathrm{H}_{49} \mathrm{O}_{23}$ & 933.2654 & $\begin{array}{l}771,433 \\
271^{\mathrm{c}}\end{array}$ & {$[22,32]$} \\
\hline 9 & 39.45 & 12.06 & $\begin{array}{l}\text { Pelaragonin-3- } \\
\text { ( } p \text {-coumaroyl)diglucoside-5-glucoside }\end{array}$ & $\mathrm{C}_{42} \mathrm{H}_{47} \mathrm{O}_{22}$ & 903.2560 & $\begin{array}{l}741,433 \\
271^{\mathrm{c}}, 149\end{array}$ & {$[22,32]$} \\
\hline 10 & 42.34 & 11.25 & $\begin{array}{l}\text { Pelaragonin-3-(caffeoyl) } \\
\text { diglucoside-5-(malonoyl) } \\
\text { glucoside }\end{array}$ & $\mathrm{C}_{45} \mathrm{H}_{49} \mathrm{O}_{26}$ & 1005.2512 & $\begin{array}{l}757,519 \\
271^{c}, 149\end{array}$ & {$[22,32]$} \\
\hline 11 & 45.44 & 10.61 & $\begin{array}{l}\text { Pelaragonin-3-(caffeoyl) } \\
\text { glucoside-5-glucoside }\end{array}$ & $\mathrm{C}_{42} \mathrm{H}_{47} \mathrm{O}_{23}$ & 919.2508 & $\begin{array}{l}757,433 \\
271^{\mathrm{c}}\end{array}$ & {$[22,32]$} \\
\hline 12 & 46.85 & 7.43 & $\begin{array}{l}\text { Pelaragonin-3-diglucoside-5- } \\
\text { (malonoyl)glucoside }\end{array}$ & $\mathrm{C}_{36} \mathrm{H}_{43} \mathrm{O}_{23}$ & 843.2197 & $519,271^{\mathrm{c}}$ & {$[22,32]$} \\
\hline 13 & 53.35 & $\mathrm{n} / \mathrm{d}$ & $\begin{array}{l}\text { Pelaragonin-3-(feruloyl) } \\
\text { triglucoside-5-(malonoyl) } \\
\text { glucoside }\end{array}$ & $\mathrm{C}_{52} \mathrm{H}_{61} \mathrm{O}_{31}$ & 1181.3163 & $933,271^{c}$ & {$[22,32]$} \\
\hline 14 & 54.21 & 12.79 & Pelaragonin-3-diglucoside-5-glucoside & $\mathrm{C}_{33} \mathrm{H}_{41} \mathrm{O}_{20}$ & 757.2192 & $\begin{array}{l}595,433 \\
271^{\mathrm{c}}\end{array}$ & {$[22,32]$} \\
\hline 15 & 54.84 & 5.60 & $\begin{array}{l}\text { Pelaragonin-3- } \\
\text { ( } p \text {-coumaroyl)triglucoside-5- } \\
\text { (malonoyl)glucoside }\end{array}$ & $\mathrm{C}_{51} \mathrm{H}_{59} \mathrm{O}_{30}$ & 1151.3075 & $519,271^{c}$ & {$[22,32]$} \\
\hline \multicolumn{8}{|l|}{ Red grape skins } \\
\hline 1 & 17.81 & $\mathrm{n} / \mathrm{d}$ & $\begin{array}{l}\text { Acetaldehyde derivative of } \\
\text { malvidin-3-actylglucoside } \\
\text { (Acetylvitisin B) }\end{array}$ & $\mathrm{C}_{27} \mathrm{H}_{27} \mathrm{O}_{13}$ & 559.1452 & $355^{c}$ & {$[6,7,22]$} \\
\hline 2 & 20.58 & 14.25 & $\begin{array}{l}\text { Acetaldehyde derivative of } \\
\text { malvidin-3-( } p \text {-coumaroyl) } \\
\text { glucoside(Coumaroylvitisin B) }\end{array}$ & $\mathrm{C}_{34} \mathrm{H}_{31} \mathrm{O}_{14}$ & 663.1714 & $355^{c}$ & {$[6,7,22]$} \\
\hline 3 & 22.04 & 12.97 & $\begin{array}{l}\text { Acetaldehyde derivative of } \\
\text { malvidin-3-glucoside (Vitisin B) }\end{array}$ & $\mathrm{C}_{25} \mathrm{H}_{25} \mathrm{O}_{12}$ & 517.1373 & $355^{c}$ & {$[6,7,22]$} \\
\hline 4 & 23.94 & 12.91 & Malvidin-3-acetylglucoside & $\mathrm{C}_{25} \mathrm{H}_{27} \mathrm{O}_{13}$ & 535.1453 & $\begin{array}{l}331^{c}, 315 \\
242\end{array}$ & {$[6,7,22,47]$} \\
\hline 5 & 24.86 & 12.39 & Peonidin-3-acetylglucoside & $\mathrm{C}_{24} \mathrm{H}_{25} \mathrm{O}_{12}$ & 505.1346 & $301^{\mathrm{c}}, 286$ & {$[6,7,22,47]$} \\
\hline 6 & 27.07 & 10.65 & $\begin{array}{l}\text { Pyruvic acid derivative of } \\
\text { malvidin-3-acetylglucoside } \\
\text { (Acetylvitisin A) }\end{array}$ & $\mathrm{C}_{28} \mathrm{H}_{27} \mathrm{O}_{15}$ & 603.1350 & $399^{c}$ & {$[6,7,22]$} \\
\hline 7 & 28.46 & 15.91 & Malvidin-3-(p-coumaroyl) glucoside & $\mathrm{C}_{32} \mathrm{H}_{31} \mathrm{O}_{14}$ & 639.1717 & $331^{c}$ & {$[6,7,22,47]$} \\
\hline 8 & 28.97 & 15.49 & Peonidin-3-(p-coumaroyl) glucoside & $\mathrm{C}_{31} \mathrm{H}_{29} \mathrm{O}_{13}$ & 609.1613 & $\begin{array}{l}301^{\mathrm{c}}, 286 \\
258,147\end{array}$ & {$[6,7,22,47]$} \\
\hline 9 & 31.00 & 13.08 & $\begin{array}{l}\text { Pyruvic acid derivative of } \\
\text { malvidin-3- }(p \text {-coumaroyl) glucoside } \\
\text { (Coumaroylvitisin } \mathrm{A})\end{array}$ & $\mathrm{C}_{35} \mathrm{H}_{31} \mathrm{O}_{16}$ & 707.1609 & $399^{c}$ & {$[6,7,22]$} \\
\hline 10 & 33.13 & 11.04 & Petunidin-3-acetylglucoside & $\mathrm{C}_{24} \mathrm{H}_{25} \mathrm{O}_{13}$ & 521.1296 & $317^{c}, 302$ & {$[6,7,22,47]$} \\
\hline 11 & 33.79 & 8.85 & Malvidin-3-glucoside & $\mathrm{C}_{23} \mathrm{H}_{25} \mathrm{O}_{12}$ & 493.1354 & $\begin{array}{l}331^{\mathrm{c}}, 315 \\
287,149\end{array}$ & {$[6,7,22,47]$} \\
\hline 12 & 34.08 & 13.78 & Malvidin-3-(caffeoyl) glucoside & $\mathrm{C}_{32} \mathrm{H}_{31} \mathrm{O}_{15}$ & 655.1663 & $\begin{array}{l}331^{\mathrm{c}}, 242 \\
147\end{array}$ & {$[6,7,22,47]$} \\
\hline 13 & 34.88 & 8.24 & Peonidin-3-glucoside & $\mathrm{C}_{22} \mathrm{H}_{23} \mathrm{O}_{11}$ & 463.1239 & $\begin{array}{l}301^{\mathrm{c}}, 286 \\
258,201\end{array}$ & {$[6,7,22,47]$} \\
\hline 14 & 35.32 & 13.24 & Peonidin-3-(caffeoyl) glucoside & $\mathrm{C}_{31} \mathrm{H}_{29} \mathrm{O}_{14}$ & 625.1551 & $301^{c}, 149$ & {$[6,7,22,47]$} \\
\hline 15 & 36.41 & 9.78 & $\begin{array}{l}\text { Malvidin-3-glucoside-pyruvic acid } \\
\text { (Vitisin A) }\end{array}$ & $\mathrm{C}_{26} \mathrm{H}_{25} \mathrm{O}_{14}$ & 561.1244 & $399^{c}$ & {$[6,7,22]$} \\
\hline 16 & 37.51 & 14.02 & $\begin{array}{l}\text { Petunidin-3-( } p \text {-coumaroyl }) \\
\text { glucoside }\end{array}$ & $\mathrm{C}_{31} \mathrm{H}_{29} \mathrm{O}_{14}$ & 625.1567 & $317^{c}$ & {$[6,7,22,47]$} \\
\hline 17 & 38.17 & 13.42 & Cyanidin-3-(p-coumaroyl) glucoside & $\mathrm{C}_{30} \mathrm{H}_{27} \mathrm{O}_{13}$ & 595.1458 & $287^{c}, 149$ & {$[6,7,22,47]$} \\
\hline 18 & 39.18 & 12.13 & Petunidin-3-(caffeoyl) glucoside & $\mathrm{C}_{31} \mathrm{H}_{29} \mathrm{O}_{15}$ & 641.1524 & $317^{c}$ & {$[6,7,22,47]$} \\
\hline 19 & 41.38 & 9.18 & Delphinidin-3-acetylglucoside & $\mathrm{C}_{23} \mathrm{H}_{23} \mathrm{O}_{13}$ & 507.1139 & $303^{c}$ & {$[6,7,22,47]$} \\
\hline 20 & 42.5 & 12.37 & Delphinidin-3-( $p$-coumaroyl)glucoside & $\mathrm{C}_{30} \mathrm{H}_{27} \mathrm{O}_{14}$ & 611.1413 & $303^{c}$ & {$[6,7,22,47]$} \\
\hline 21 & 43.27 & 7.25 & Petunidin-3-glucoside & $\mathrm{C}_{22} \mathrm{H}_{23} \mathrm{O}_{12}$ & 479.1191 & $317^{c}$ & {$[6,7,22,47]$} \\
\hline 22 & 44.80 & 6.47 & Cyanidin-3-glucoside & $\mathrm{C}_{21} \mathrm{H}_{21} \mathrm{O}_{11}$ & 449.1083 & $287^{c}$ & {$[6,7,22,47]$} \\
\hline 23 & 53.34 & 5.51 & Delphinidin-3-glucoside & $\mathrm{C}_{21} \mathrm{H}_{21} \mathrm{O}_{12}$ & 465.1046 & $303^{c}$ & {$[6,7,22,47]$} \\
\hline \multicolumn{8}{|l|}{ Red cabbage } \\
\hline 1 & 30.14 & 13.24 & $\begin{array}{l}\text { Cyanidin-3-(sinapoyl) } \\
\text { glucoside-5-glucoside }\end{array}$ & $\mathrm{C}_{38} \mathrm{H}_{41} \mathrm{O}_{20}$ & 817.2172 & $449,287^{c}$ & {$[22,32,44]$} \\
\hline 2 & 31.91 & 12.92 & $\begin{array}{l}\text { Cyanidin-3-(sinapoyl) } \\
\text { (sinapoyl)diglucoside-5-glucoside }\end{array}$ & $\mathrm{C}_{55} \mathrm{H}_{61} \mathrm{O}_{29}$ & 1185.3298 & $\begin{array}{l}1023,449 \\
287^{c}\end{array}$ & {$[22,32,41,42,44]$} \\
\hline 3 & 32.26 & 12.46 & $\begin{array}{l}\text { Cyanidin-3-( sinapoyl) } \\
\text { (feruloyl)diglucoside-5-glucoside }\end{array}$ & $\mathrm{C}_{54} \mathrm{H}_{59} \mathrm{O}_{28}$ & 1155.3190 & $\begin{array}{l}993,899 \\
449,287^{c}\end{array}$ & {$[22,32,42,44]$} \\
\hline 4 & 33.58 & 12.48 & $\begin{array}{l}\text { Cyanidin-3-(feruloyl) } \\
\text { (feruloyl)diglucoside-5-glucoside }\end{array}$ & $\mathrm{C}_{53} \mathrm{H}_{57} \mathrm{O}_{27}$ & 1125.3094 & $\begin{array}{l}963,449 \\
287^{c}\end{array}$ & {$[22,32,42,44]$} \\
\hline 5 & 45.84 & 11.58 & $\begin{array}{l}\text { Cyanidin-3-(feruloyl) } \\
\text { diglucoside-5-glucoside }\end{array}$ & $\mathrm{C}_{43} \mathrm{H}_{49} \mathrm{O}_{24}$ & 949.2615 & $\begin{array}{l}787,449 \\
287^{c}\end{array}$ & {$[22,32,43,44]$} \\
\hline $6^{\mathrm{A}}$ & 45.84 & 11.58 & $\begin{array}{l}\text { Cyanidin-3-(sinapoyl) } \\
\text { diglucoside-5-glucoside }\end{array}$ & $\mathrm{C}_{44} \mathrm{H}_{51} \mathrm{O}_{25}$ & 979.2717 & $449,287^{c}$ & {$[22,32,43,44]$} \\
\hline $6^{\mathrm{B}}$ & 45.84 & 6.51 & $\begin{array}{l}\text { Cyanidin-3-(sinapoyl) } \\
\text { diglucoside-5-glucoside }\end{array}$ & $\mathrm{C}_{44} \mathrm{H}_{51} \mathrm{O}_{25}$ & 979.2694 & $\begin{array}{l}817,449 \\
287^{c}\end{array}$ & {$[22,32,43,44]$} \\
\hline
\end{tabular}


Table 2 (Continued)

\begin{tabular}{|c|c|c|c|c|c|c|c|}
\hline Compound number ${ }^{\mathrm{a}}$ & HILIC $t_{R}^{\mathrm{b}}(\mathrm{min})$ & $\begin{array}{l}\mathrm{RP}-\mathrm{LC} t_{R}^{\mathrm{b}} \\
(\mathrm{min})\end{array}$ & Identity & $\begin{array}{l}\text { Molecular } \\
\text { formula }[\mathrm{M}]^{+}\end{array}$ & $\begin{array}{l}\text { Experimental } \\
\text { mass }(m / z)\end{array}$ & $\begin{array}{l}\mathrm{MS}^{\mathrm{E}} \\
\text { Fragments }\end{array}$ & References \\
\hline 7 & 47.20 & 11.05 & $\begin{array}{l}\text { Cyanidin-3-( } p \text {-coumaroyl) } \\
\text { diglucoside-5-glucoside }\end{array}$ & $\mathrm{C}_{42} \mathrm{H}_{47} \mathrm{O}_{23}$ & 919.2502 & $\begin{array}{l}757,449 \\
287^{c}\end{array}$ & {$[22,32,44]$} \\
\hline 8 & 51.37 & 10.08 & $\begin{array}{l}\text { Cyanidin-3-(sinapoyl) } \\
\text { (feruloyl)triglucoside-5-glucoside }\end{array}$ & $\mathrm{C}_{60} \mathrm{H}_{69} \mathrm{O}_{33}$ & 1317.3732 & $\begin{array}{l}1155,449 \\
287^{c}\end{array}$ & {$[22,32,44]$} \\
\hline 9 & 51.37 & 10.34 & $\begin{array}{l}\text { Cyanidin-3-(sinapoyl) } \\
\text { (sinapoyl)triglucoside-5-glucoside }\end{array}$ & $\mathrm{C}_{61} \mathrm{H}_{71} \mathrm{O}_{34}$ & 1347.3806 & $\begin{array}{l}1185,449 \\
287^{c}\end{array}$ & {$[22,32,44]$} \\
\hline 10 & 52.26 & 9.74 & $\begin{array}{l}\text { Cyanidin-3-(sinapoyl) } \\
\text { ( } p \text {-coumaroyl) } \\
\text { triglucoside-5-glucoside }\end{array}$ & $\mathrm{C}_{59} \mathrm{H}_{67} \mathrm{O}_{32}$ & 1287.3625 & $\begin{array}{l}1125,449 \\
287^{c}\end{array}$ & {$[22,32,44]$} \\
\hline 11 & 59.39 & $\mathrm{n} / \mathrm{d}$ & $\begin{array}{l}\text { Cyanidin-3-(caffeoyl) } \\
\text { diglucoside-5-glucoside }\end{array}$ & $\mathrm{C}_{42} \mathrm{H}_{47} \mathrm{O}_{24}$ & 935.2427 & $\begin{array}{l}773,449 \\
287^{c}\end{array}$ & {$[22,32,44]$} \\
\hline $12^{\mathrm{A}}$ & 59.10 & 6.49 & $\begin{array}{l}\text { Cyanidin-3-(feruloyl) } \\
\text { triglucoside-5-glucoside }\end{array}$ & $\mathrm{C}_{49} \mathrm{H}_{59} \mathrm{O}_{29}$ & 1111.3146 & $\begin{array}{l}949,449 \\
287^{c}\end{array}$ & {$[22,32,44]$} \\
\hline $13^{A}$ & 62.20 & $\mathrm{n} / \mathrm{d}$ & $\begin{array}{l}\text { Cyanidin-3-(sinapoyl) } \\
\text { triglucoside-5-glucoside }\end{array}$ & $\mathrm{C}_{50} \mathrm{H}_{61} \mathrm{O}_{30}$ & 1141.3247 & $\begin{array}{l}979,449, \\
287^{c}\end{array}$ & {$[22,32,44]$} \\
\hline 14 & 62.20 & 4.88 & Cyanidin-3-diglucoside-5-glucoside & $\mathrm{C}_{33} \mathrm{H}_{41} \mathrm{O}_{21}$ & 773.2141 & $\begin{array}{l}611,449 \\
287^{c}\end{array}$ & {$[22,32,44]$} \\
\hline $12^{\mathrm{B}}$ & 66.90 & 8.79 & $\begin{array}{l}\text { Cyanidin-3-(feruloyl) } \\
\text { triglucoside-5-glucoside }\end{array}$ & $\mathrm{C}_{49} \mathrm{H}_{59} \mathrm{O}_{29}$ & 1111.3127 & $\begin{array}{l}949,449 \\
287^{c}\end{array}$ & {$[22,32,44]$} \\
\hline $13^{\mathrm{B}}$ & 66.90 & 9.01 & $\begin{array}{l}\text { Cyanidin-3-(sinapoyl) } \\
\text { triglucoside-5-glucoside }\end{array}$ & $\mathrm{C}_{50} \mathrm{H}_{61} \mathrm{O}_{30}$ & 1141.3220 & $\begin{array}{l}979,449 \\
287^{c}\end{array}$ & {$[22,32,44]$} \\
\hline 15 & 68.10 & 8.50 & $\begin{array}{l}\text { Cyanidin-3-( } p \text {-coumaroyl) } \\
\text { triglucoside-5-glucoside }\end{array}$ & $\mathrm{C}_{48} \mathrm{H}_{57} \mathrm{O}_{28}$ & 1081.3055 & $\begin{array}{l}919,449 \\
287^{c}\end{array}$ & {$[22,32,44]$} \\
\hline
\end{tabular}

a Peak numbers correspond to Figs. 2-5.

b $t_{0}$ times for HILIC and RP-LC separations were 7.0 and $0.92 \mathrm{~min}$, respectively.

c Base peak ion in $\mathrm{MS}^{\mathrm{E}}$ or MS/MS spectrum.

d Peak numbers marked with superscript letters A, B, C or D denote isomeric compounds with identical MS characteristics.

isomers differ in their acylation patterns, although the exact differences in acylation positions could not be determined based on MS data. The fact that some of these isomeric compounds co-eluted in one dimension (for example $5^{\mathbf{C}}$ and $5^{\mathbf{D}}$ in HILIC, and $\mathbf{6}^{\mathbf{A}}$ and ${ }_{6} \mathrm{C}$ in RP-LC) and cannot be distinguished by MS, emphasises the importance of improved chromatographic separation offered by HILIC $\times$ RP-LC.

\subsubsection{Red grape skins}

Red grape skin anthocyanins comprise mainly the anthocyanidin-monoglucosides and their acetyl-, p-coumaroyl-, and caffeoyl-derivatives. A total of 23 anthocyanins and derivatives were identified in this sample (Fig. 4). Grape anthocyanins have a characteristic elution order in RP-LC, with the -glucosides eluting before acetyl-glucosides, which elute before the $p$-coumaroylglucosides. For each of these classes, the relative elution order is based on the anthocyanidin bases according to the following sequence: delphinidin, cyanidin, petunidin, peonidin and malvidin. Partial overlap between the different classes of grape anthocyanins does occur, especially for the acylated compounds (the caffeoyl derivatives for example elute between the -acetyl and - $p$-coumaroyl derivatives). As a consequence, co-elution between compounds of different classes may occur. For example, delphinidin-3-( $p$-coumaroyl)glucoside (20) and peonidin-3acetylglucoside (5) were found to co-elute in RP-LC. The retention order of anthocyanins according to both the anthocyanidin base and the nature of acylation are somewhat different in HILIC, resulting in the clear separation of these compounds in HILIC.

On the other hand, the non-acylated and caffeoyl derivatives of malvidin and peonidin (i.e. malvidin-3-glucoside (11) and malvidin-3-(caffeoyl)glucoside (12), peonidin-3-glucoside (13) and peonidin-3-(caffeoyl)glucoside (14)) were not resolved in HILIC but were separated in RP-LC as a result of the higher retention of caffeoyl derivatives in this mode. The pyranoanthocyanin Vitisin B (3), resulting from the cycloaddition of acetaldehyde to malvidin3 -glucoside $[7,45]$, was also observed in the contour plot at lower HILIC and higher RP-LC retention, respectively, than malvidin-3glucoside (11) [7,45].

\subsubsection{Red cabbage}

A total of 18 anthocyanins were identified in red cabbage (Fig. 5, Table 2) based on relative RP-LC retention times, MS and MS ${ }^{\mathrm{E}}$ data compared to previous literature reports [32,40]. Only cyanidin derivatives were found in this sample, with variation stemming from different acylation patterns of cyanidin-3-(di/tri)glucoside5 -glucosides $[32,33,46]$. Several isomeric structures which could not be distinguished based on MS data (compounds $\mathbf{6}^{\mathbf{A}, \mathbf{B}}, \mathbf{1 2}^{\mathbf{A}, \mathbf{B}}$ and $13^{\mathbf{A}, \mathbf{B}}$ ) were also detected in red cabbage.

The general elution pattern for red cabbage anthocyanins is based on the number of glycosidic groups as well as the type and number of acylated substituents. The tetra-glycosidic species display higher retention in HILIC than the corresponding tri-glycosides [22]; the opposite is observed in RP-LC. Furthermore, variation in the nature of the acyl-groups allowed partial separation of red cabbage anthocyanins in HILIC and clear separation in RP-LC. For example, for the same degree of glycosylation, the elution order in RP-LC is p-coumaroyl<-feruloyl<-sinapoyl, while in HILIC the retention order is roughly opposite, although the -sinapoyl and -feruloyl derivatives are not resolved in this mode. As a consequence, cyanidin-3-(sinapoyl)(sinapoyl)diglucoside-5-glucoside (2) and cyanidin-3-(sinapoyl)(feruloyl)diglucoside-5-glucoside (3), cyanidin-3-(sinapoyl)(feruloyl)triglucoside-5-glucoside (8) and cyanidin-3-(sinapoyl)(sinapoyl)triglucoside-5-glusoside (9) as well as cyanidin-3-(feruloyl)triglucoside-5-glucoside $\left(\mathbf{1 2}^{\mathbf{B}}\right)$ and cyanidin-3-(sinapoyl)triglucoside-5-glucoside $\left(\mathbf{1 3}^{\mathbf{B}}\right)$ all co-elute in HILIC but were separated in the RP-LC dimension due to better differentiation according to the nature of acyl groups in this mode.

Cyanidin-3-(feruloyl)diglucoside-5-glucoside (5) and cyanidin3-(sinapoyl)diglucoside-5-glucoside $\left(\mathbf{6}^{\mathbf{A}}\right)$ co-eluted in both dimensions, whereas cyanidin-3-(feruloyl)triglucoside-5-glucoside $\left(\mathbf{1 2}^{\mathbf{A}}\right)$ and cyanidin-3-(sinapoyl)diglucoside-5-glucoside $\left(\mathbf{6}^{\mathbf{B}}\right)$ co-eluted in RP-LC, but were separated in HILIC based on their different degree of glycosylation.

\subsubsection{Structured elution patterns of anthocyanins}

The primary reason for performing $\mathrm{LC} \times \mathrm{LC}$ analysis of anthocyanins is to combine the selectivity of two independent separation 

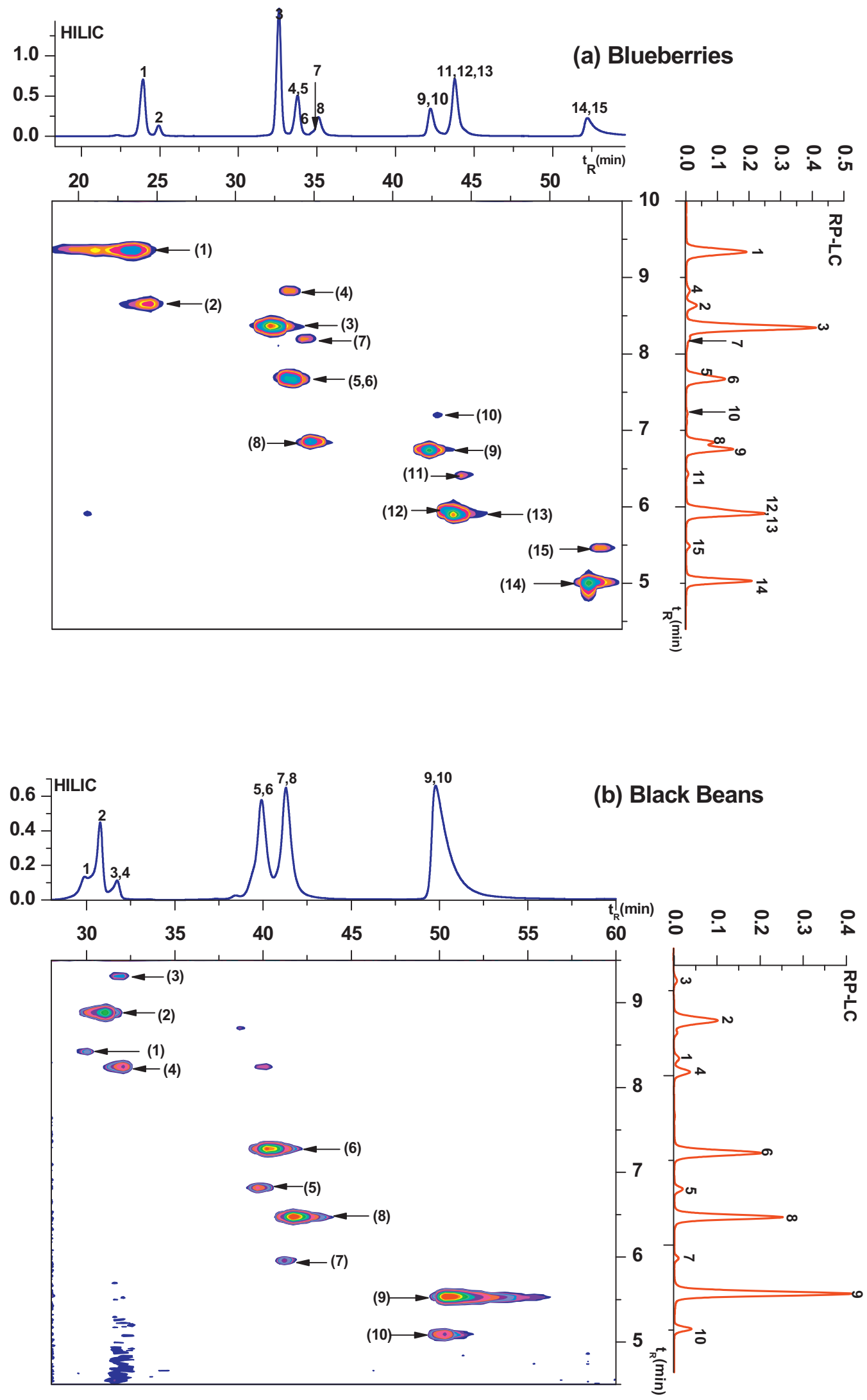

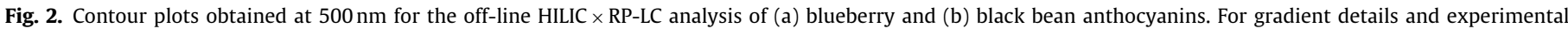
conditions, refer to Section 2.4.1. Peak numbers correspond to Table 2. 


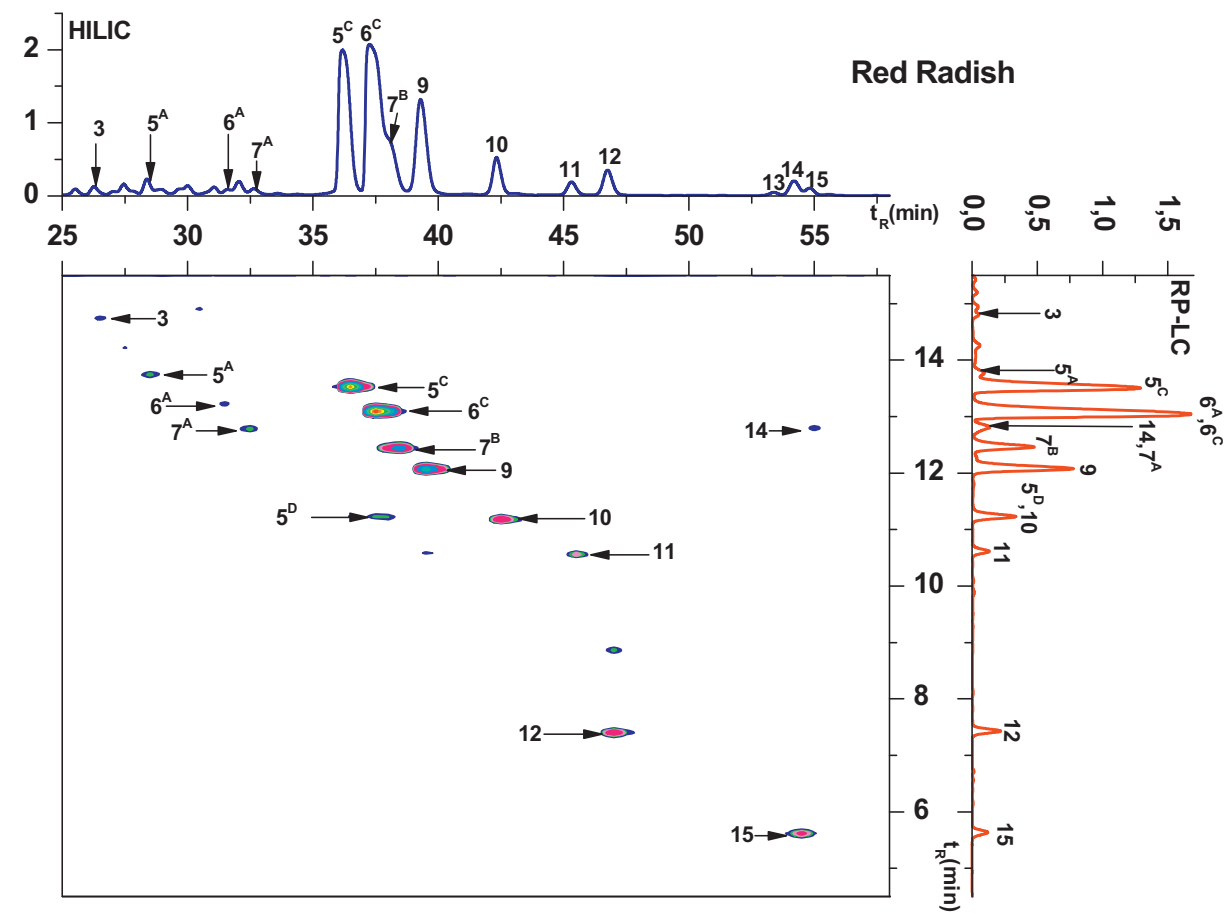

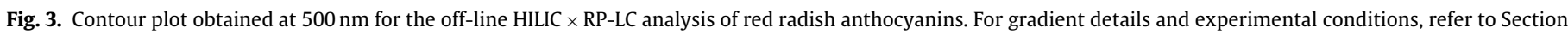
2.4.1. Peak numbers correspond to Table 2 .

modes to obtain enhanced resolution. From the precedent discussion and the contour plots presented in Figs. 2-5 the improved resolution offered by a HILIC $\times$ RP-LC separation is evident. In fact, with the exception of the RP-LC analysis of black beans, neither 1-dimensional HILIC nor RP-LC provided complete separation of anthocyanins in any of the other samples (Supplementary information, Figs. S1 and S2). This is also true for dedicated RP-LC methods developed for example for the analysis of red grape skins and blueberries [8], red radish and black beans [32] and red cabbage [44].

Another noteworthy advantage of HILIC $\times$ RP-LC separation is the group-type separation afforded by the combination of these separation modes, as highlighted for grape skin and red cabbage anthocyanins by the dotted lines in Figs. 4 and 5 .

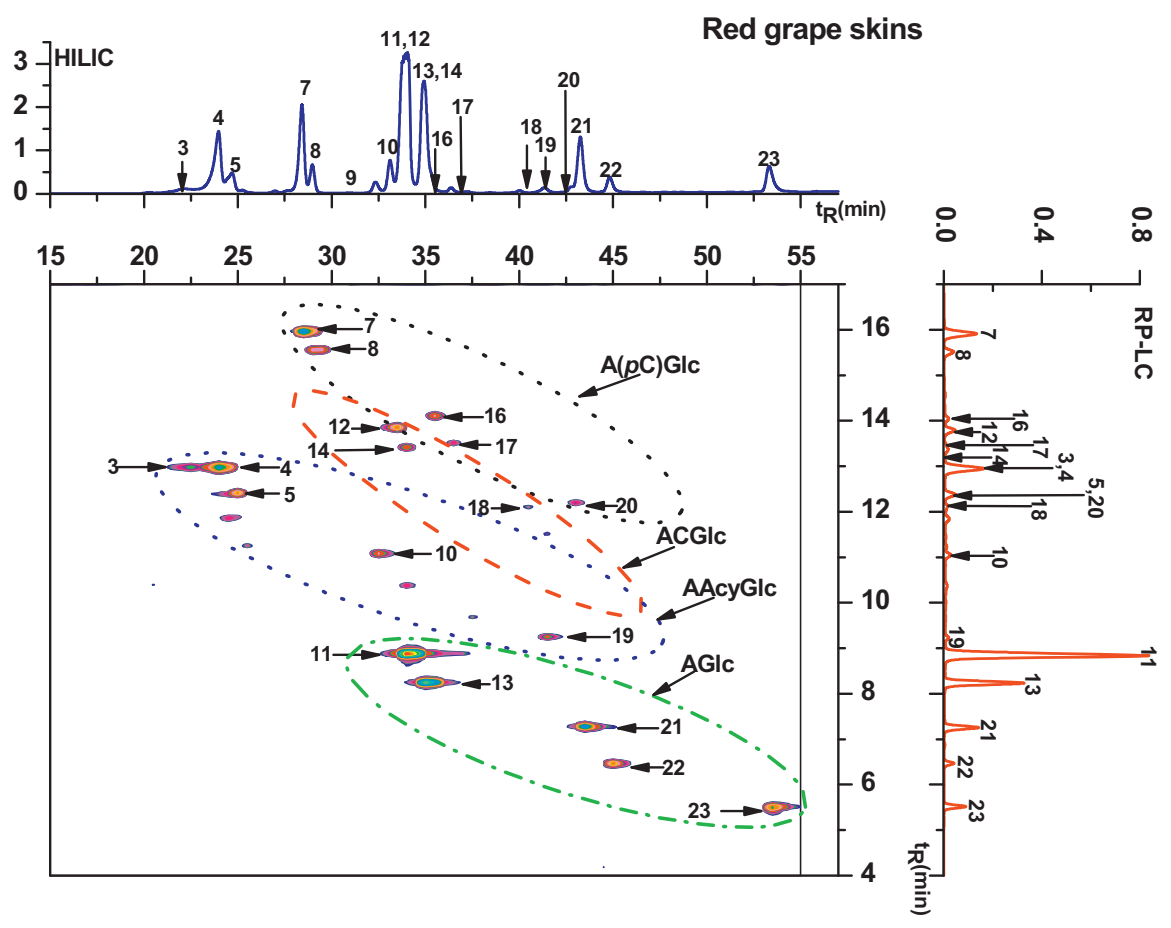

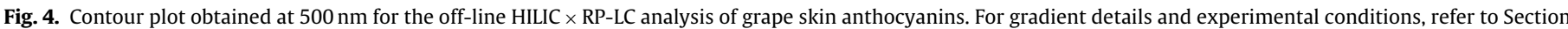

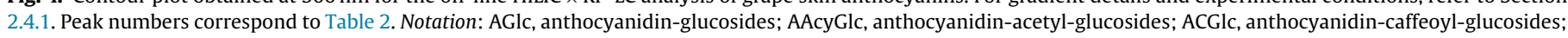
$\mathrm{A}(p \mathrm{C}) \mathrm{Glc}$, anthocyanidin-( $p$-coumaroyl)glucosides. 


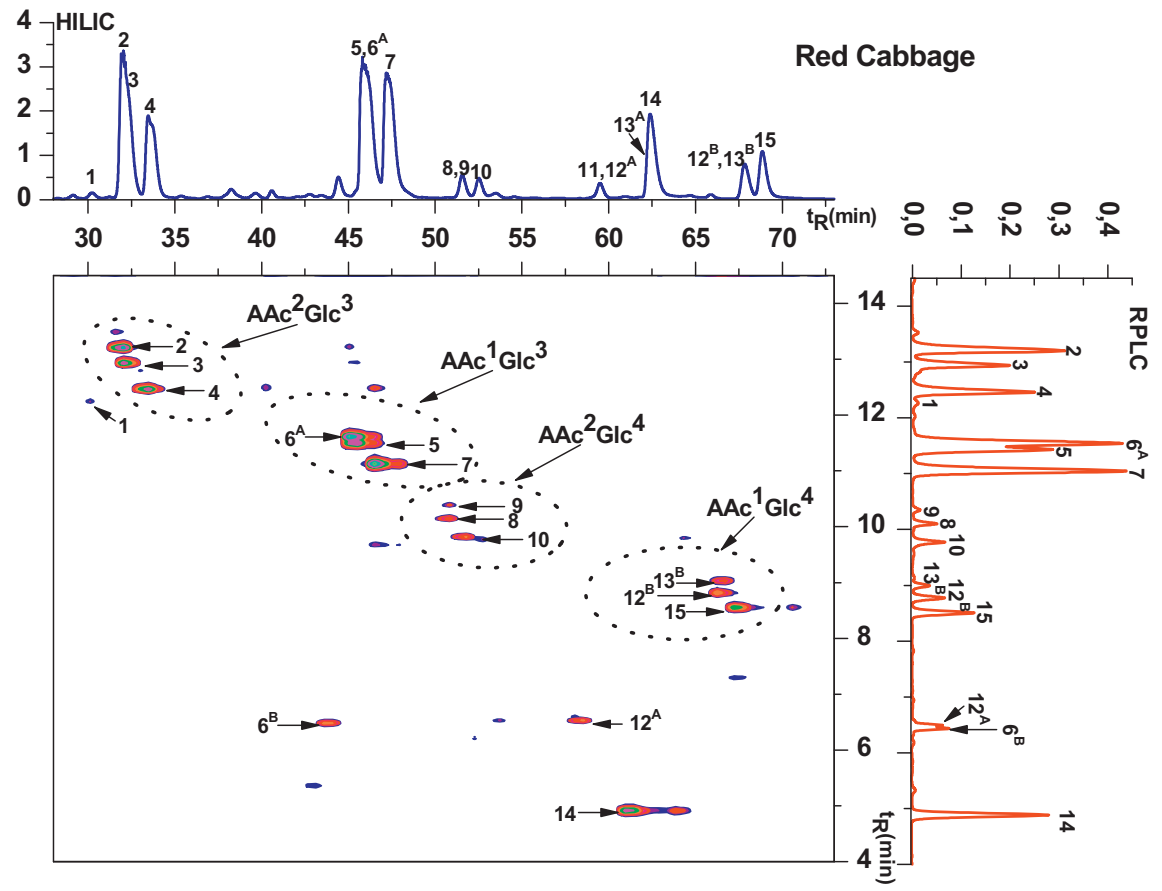

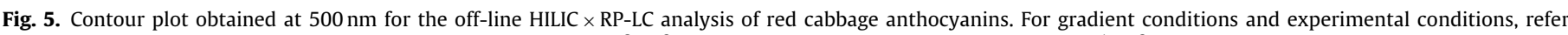

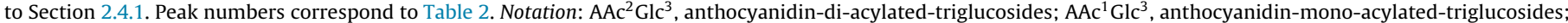
$\mathrm{AAc}^{2} \mathrm{Glc}^{4}$, anthocyanidin-di-acylated-tetra-glucosides; $\mathrm{AAc}^{1} \mathrm{Glc}^{4}$, anthocyanidin-mono-acylated-tetra-glucosides.

In the case of grape skins, anthocyanidin-3-glucosides elute in the region of high HILIC retention ( $\sim 30-55$ min Fig. 4) and low RP-LC retention ( $\sim 5-9$ min). Relative to this group, anthocyanidin-acetyl-glucosides elute in the region of moderately high RP-LC (9-13 min) and lower HILIC retention ( $20-47 \mathrm{~min})$. Anthocyanidin-caffeoyl-glucosides elute amongst the acetylglucosides but show higher retention in both dimensions. Finally, anthocyanidin- $p$-coumaroyl-glucosides were the most retained compounds in RP-LC and were moderately well retained in HILIC. Within each of these classes, anthocyanins are grouped according to the polarity of the anthocyanidin base, with the elution order being opposite in HILIC to that observed in RP-LC, but also differing in the relative retention between different anthocyanidin bases.

For the higher acylated anthocyanidin- tri- and tetra-glucosides in red cabbage the following grouping was observed: the anthocyanidin-di-acylated-tri-glucosides were the least retained group of compounds in the HILIC dimension ( $31-35 \mathrm{~min})$, and were highly retained in the second dimension ( $\sim 12-14 \mathrm{~min})$ (Fig. 5). Relative to this group the anthocyanidin-mono-acylatedtri-glucosides were more retained in the HILIC dimension ( $\sim 45-50 \mathrm{~min})$ and less in the RP-LC dimension ( $11-12 \mathrm{~min}$ ). Compared to these two groups, the anthocyanidin-di-acylatedtetra-glucosides displayed higher retention in HILIC ( $\sim 51-55 \mathrm{~min})$, indicating that the degree of glycosylation is more important than the degree of acylation in HILIC. Analogous to the tri-glucosides, the mono-acylated-tetra-glucoside derivatives, the most retained group of compounds in the first dimension ( $\sim 65-70 \mathrm{~min})$, eluted after the di-acylated-tetra-glucosides. In general the opposite behaviour is observed in the second dimension, with RP-LC retention increasing with an increase in the degree of acylation and a decrease in the degree of glycosylation.

The red radish sample also contains highly acylated anthocyanidins, and, similar to red cabbage, their relative retention was primarily determined by the degree of glycosylation (especially important in HILIC) and acylation (influential in RP-LC). For red radish anthocyanins, however, the presence or absence of malonoyl-groups on the 5-O-glucoside added additional selectivity: this form of acylation results in lower HILIC and higher RP-LC retention (Fig. 3, Table 2). For both red cabbage and red radish anthocyanins, for the same degree of glycosylation and acylation, the elution order in both dimensions was based on the polarity of the acyl group.

This relatively structured elution pattern observed for the anthocyanins is advantageous, since it allows tentative assignment of the degree and/or nature of acylation/glycosylation of unidentified peaks according to their position in the contour plot relative to known compounds. This is especially helpful when compounds with similar masses and fragmentation patterns are encountered, such as for example pelaragonidin-3-( $p$ coumaroyl)diglucoside-5(malonoyl)glucoside and pelaragonidin3-(feruloyl)diglucoside-5(malonoyl)glucoside isomers found in red radish and cyanidin-3-(sinapoyl)diglucoside-5-glucoside and cyanidin-3-(feruloyl)triglucoside-5-glucoside isomers in red cabbage. It is relevant to note here that the reproducibility of off-line HILIC $\times$ RP-LC separation has been shown to be excellent [27].

\subsection{Evaluation of the performance of off-line HILIC $\times R P$-LC separation of anthocyanins}

The performance of LC $\times$ LC separations is commonly evaluated in terms of peak capacity, $n_{\mathrm{c}}$, defined as the number of peaks that can theoretically be separated within a given analysis time [34,35]. Ideally the peak capacity of an LC $\times$ LC separation is equal to the product of the peak capacities of the first $\left({ }^{1} n_{\mathrm{c}}\right)$ and second $\left({ }^{2} n_{\mathrm{c}}\right)$ dimensions [36,37]. To determine the peak capacities of both HILIC and RP-LC separations, the following relationship was used $[26,38]$ :

$n_{\mathrm{c}, 1 D}=1+\frac{t_{\mathrm{g}}}{1 / n \sum_{1}^{n} w_{\mathrm{b}}}$

where $t_{\mathrm{g}}$ is the gradient time and $w_{\mathrm{b}}$ is the peak width at baseline, averaged for $n$ number of peaks. The HILIC separation provided peak capacities ranging between 46 and 78 for the analysed samples, with the largest value obtained for red radish (Table 3 ). These values represent relatively good performance, especially considering 
Table 3

Summary of parameters used to calculate the practical peak capacity of off-line HILIC $\times$ RP-LC analysis of anthocyanins.

\begin{tabular}{|c|c|c|c|c|c|}
\hline Parameters & Blueberries & Black beans & Grape skins & Red cabbage & Red radish \\
\hline HILIC peak capacity ${ }^{\mathrm{a}},{ }^{1} n_{\mathrm{c}}$ & 58 & 46 & 65 & 60 & 78 \\
\hline RP peak capacity (average) ${ }^{\mathrm{a}},{ }^{2} n_{\mathrm{c}}$ & 176 & 196 & 109 & 171 & 159 \\
\hline Surface coverage ${ }^{\mathrm{b}},\left(\mathrm{SC}_{\mathrm{s}}, f_{\mathrm{c}}\right)$ & 0.563 & 1.00 & 0.639 & 0.625 & 0.938 \\
\hline Surface coverage ${ }^{c},\left(\mathrm{SC}_{\mathrm{CH}}, f_{\mathrm{c}}\right)$ & 0.254 & 0.733 & 0.482 & 0.488 & 0.740 \\
\hline Theoretical peak capacity ${ }^{\mathrm{d}}, n_{\mathrm{c}, 2 \mathrm{D}}$ & 10208 & 9016 & 7085 & 10260 & 12402 \\
\hline Under-sampling correction factor ${ }^{\mathrm{e}}, \beta$ & 1.15 & 1.16 & 1.22 & 1.10 & 1.32 \\
\hline Practical peak capacity ${ }^{\mathrm{f}}, n_{\mathrm{c}, 2 \mathrm{D}}$ & 4997 & 7772 & 3711 & 5830 & 8813 \\
\hline Practical peak capacity ${ }^{g}, n^{*}{ }_{c, 2 \mathrm{D}}$ & 2255 & 5697 & 2799 & 4552 & 6953 \\
\hline
\end{tabular}

a Calculated according to [38], Eq. (1).

b Calculated according to Stoll et al. [52] (supplementary information Fig. S3).

c Calculated according to the convex hull method [54] (supplementary information Fig. S4).

${ }^{\text {d }} n_{\mathrm{c}, 2 \mathrm{D}}={ }^{1} n_{\mathrm{c}} \times{ }^{2} n_{\mathrm{c}}$.

e $\beta=$ calculated according to Li et al. [49], Eq. (3).

f Calculated according to Eq. (2) [23], corrected for lack of orthogonality by using SC [52] and under-sampling according to [49].

g Calculated according to Eq. (2) [23], corrected for lack of orthogonality by using $\mathrm{SC}_{\mathrm{CH}}$ [53] and under-sampling according to [49].

that chromatographic efficiency, and therefore peak capacities, are generally lower in HILIC compared to RP-LC [47]. Peak capacities for the second dimension RP-LC separations varied between 109 and 196 (average 163). These values represents excellent performance for a relatively short gradient, which can be ascribed to the use of a $2.6 \mu \mathrm{m}$ superficially porous phase operated under optimal conditions (low flow rate and elevated temperature), in accordance with $[9,10]$.

In order to obtain a realistic measure of the performance of HILIC $\times$ RP-LC separation of anthocyanins, the practical 2dimensional peak capacity $\left(n_{\mathrm{c}, 2 D}^{*}\right)$, taking into account both the effects of first dimension sampling rates (i.e. first dimension undersampling) and finite orthogonality, was calculated according to:

$n_{\mathrm{c}, 2 D}^{*}=\frac{{ }^{1} n_{\mathrm{c}} \times{ }^{2} n_{\mathrm{c}} \times f_{\mathrm{c}}}{\beta}$

where $\beta$ is a measure of under-sampling of first dimension peaks [48] and $f_{\mathrm{c}}$ is the fractional surface coverage of the available 2dimensional space [23].

For the off-line hyphenation of HILIC and RP-LC separations, a first dimension sampling time of $0.5 \mathrm{~min}$ was used in the current study. Considering the measured anthocyanin peak widths in HILIC, every first dimension peak was therefore sampled 2-3 times. The under-sampling correction factor, $\beta$, was calculated according to $\mathrm{Li}$ et al. [49] (Eq. (3)), and is listed for each sample in Table 3:

$\beta=\sqrt{1+3.35\left(\frac{{ }^{1} t_{\mathrm{s}} \times{ }^{1} n_{\mathrm{c}}}{{ }^{1} t_{\mathrm{g}}}\right)}$

where ${ }^{1} t_{\mathrm{s}}$ is the sampling time and ${ }^{1} t_{\mathrm{g}}$ the gradient time in the first dimension. Note that for off-line operation the sampling time replaces the second dimension cycle time $\left({ }^{2} t_{\mathrm{c}}\right)$ in the original equation reported in Ref. [49], since the latter no longer determines the sampling rate [26]. With the exception of red radish, the $\beta$ values determined for all samples indicated acceptable losses in the HILIC peak capacity due to insufficient sampling rates. A higher $\beta$ value for red radish is a result of better HILIC efficiency for the anthocyanins in this sample. The likely reasons for this have been addressed in Ref. [22]: in short, the divergent chromatographic behaviour of red radish anthocyanins may be ascribed to higher rate constants for the hydration reaction of the pelaragonidin derivatives found in this sample compared to the other anthocyanidin derivatives [50].

Secondly, the orthogonality of the HILIC $\times$ RP-LC separation of anthocyanins was also taken into account in the estimation of the practical performance of the approach. Orthogonality is a quantitative measure of efficacy of separation space utilisation in comprehensive multidimensional separations. Several orthogonality metrics have been developed $[23,51]$, although there is little consensus in literature regarding the most accurate measure of this parameter. In this contribution, the effective surface coverage (SC, $f_{c}$ ) was used as a measure of orthogonality, since this metric has a direct relationship to the 2-D peak capacity [23]. Surface coverage (SC) was estimated using two complementary methods: the 'bin counting' procedure described by Stoll et al. [52,53], and the convex hull method $\left(\mathrm{SC}_{\mathrm{CH}}\right)[53,54]$ (referred to as $\mathrm{SC}_{\mathrm{S}}$ and $\mathrm{SC}_{\mathrm{CH}}$ in Table 3, respectively). In the former approach the separation space is divided into individual 'bins', and SC is determined by the collective area of all bins containing peaks. The discretisation, in other words the choice of the number of bins, is a critical parameter. In this work, the number of bins, $B$, was selected to be equal to the number of components $N$ in each sample according to Ref. [23]. In the calculation of $\mathrm{SC}_{\mathrm{S}}$, bins that do not contain compounds but that are enclosed in the space covered by peaks are included; $\mathrm{SC}_{\mathrm{S}}$ is then obtained by dividing the number of bins within the enclosed area containing peaks by the total number of bins $[23,53]$. The convex hull method estimates SC by means of a rectangular hull $[53,54]$, where the minimum convex hull is defined as the smallest polygon that can surround a group of peaks with none of the inner hull angles exceeding $180^{\circ}$. A possible limitation of this method is that open spaces within and near the hull edges may be included because all interior hull angles are convex. It should also be noted that both $\mathrm{SC}_{\mathrm{S}}$ and $\mathrm{SC}_{\mathrm{CH}}$ are sensitive to outlying components - such data points may therefore result in overestimation of orthogonality of some two-dimensional systems found in literature.

SC values obtained using each of these methods, and the practical 2-dimensional peak capacities calculated from these values according to Eq. (2) are presented for each of the samples in Table 3 (details on the calculation of SC are presented in the Supplementary Information (Figs. S3 and S4). $\mathrm{SC}_{\mathrm{CH}}$ values are significantly lower than the corresponding $\mathrm{SC}_{\mathrm{S}}$ values for all samples. These discrepancies emphasise the importance of the metric used to estimate orthogonality in the calculation of practical peak capacities. The effect of the number of bins on $\mathrm{SC}_{\mathrm{S}}$ is especially critical for samples with few analytes, as can be seen in the larger differences between $\mathrm{SC}_{\mathrm{S}}$ and $\mathrm{SC}_{\mathrm{CH}}$ values for blueberries. Since the convex hull method does not rely on discretisation, this method was considered to provide a more reliable measure of surface coverage, and the following discussion will be limited to $n_{\mathrm{c}, 2 D}^{*}$ values obtained using $\mathrm{SC}_{\mathrm{CH}}$ data.

From Table 3, it is evident that off-line HILIC $\times$ RP-LC analysis of anthocyanins provides exceptionally high practical peak capacities compared to 1-dimensional separations (depending on the sample, 39-124× higher values). These values confirm the improved separation evident from the contour plots presented in Figs. 2-5 compared to 1-dimensional separations presented in Supplementary Information (Figs. S1 and S2). This improvement in separation 
performance is especially important considering that MS detection does not allow identification of isomeric structures.

It should be noted that this gain in performance is only achievable using off-line operation, since the longer second dimension gradient times provide high peak capacities in this dimension [26]. The off-line HILIC $\times$ RP-LC separation of a single sample requires 2310 min for the shortest HILIC gradient and 3300 min for the longest under the conditions used here. Nevertheless, peak capacity production rates (i.e. the number of peaks that can theoretically be separated per minute) are in the order of $1.4-2.5$ peaks $/ \mathrm{min}$. These values compare favourably to those obtained by 1-dimensional HILIC (0.6-0.8 peaks/min) and RP-LC (4.4-7.8 peaks/min) separations, yet provide much higher practical performance than either of these methods.

In this context, it is worth noting that while on-line $\mathrm{LC} \times \mathrm{LC}$ as a rule provides much higher peak production rates, this may not be the case for anthocyanins: the unique effect of secondary equilibria means that the chromatographic performance for these molecules is much worse under fast analysis conditions as required for online LC $\times$ LC operation. Current research is focused on exploring the possibilities of on-line HILIC $\times$ RP-LC separation of anthocyanins.

\section{Conclusions}

The analysis of anthocyanins is challenging owing to the fact that many of these compounds co-elute in one-dimensional separations and have the same molecular weight and MS fragmentation patterns. In this study, off-line comprehensive 2-dimensional HILIC $\times$ RP-LC methods for the analysis of anthocyanins were successfully developed and evaluated. HILIC $\times$ RP-LC analysis was found to offer improved resolution due to the combination of independent separation mechanisms, allowing separation of compounds co-eluting in either of the one-dimensional HPLC modes. Practical peak capacities, taking into account both first dimension under-sampling and finite orthogonality, of up to 6950 were obtained, providing peak production rates of up to 2.5 peaks $/ \mathrm{min}$. Furthermore, the group-type separation obtained by the combination of HILIC and RP-LC retention mechanisms was found to provide an effective means of facilitating compound identification.

The off-line HILIC $\times$ RP-LC method reported here proved suitable for the separation of a wide range of anthocyanins, including anthocyanidin-mono-,-di-, tri- and tetra-glycosides as well as their acylated derivatives found in blueberries, red grape skins, black beans, red cabbage and red radish.

\section{Acknowledgements}

The authors gratefully acknowledge Sasol (grant to AdV and bursary to CW), the National Research Foundation (NRF, South Africa, Grant 81830 to AdV), THRIP and the International Foundation of Science (IFS, Sweden, Grant F/4904-1 to AdV) for financial support.

\section{Appendix A. Supplementary data}

Supplementary data associated with this article can be found, in the online version, at http://dx.doi.org/10.1016/j.chroma. 2014.07.044.

\section{References}

[1] D. Strack, V. Wray, The flavonoids, in: J.B. Harborne (Ed.), Advances in Research Since 1986, Chapman and Hall, London, 1994, p. 1

[2] W. Yi, C.C. Akoh, J. Fischer, G. Krewer, Absorption of anthocyanins from blueberry extracts by Caco-2 human intestinal cell monolayers, J. Agric. Food Chem. 54 (2006) 5651-5658.
[3] M.E. Olsson, K.-E. Gustavsson, S. Anderson, A. Nilsson, R.-D. Duan, Inhibition of cancer cell proliferation in vitro by fruit and berry extracts and correlations with antioxidant levels, J. Agric. Food Chem. 52 (2004) 7264-7271.

[4] F.P. Prencipe, R. Bruni, A. Guerrini, D. Rossi, S. Benvenuti, F. Pellati, Metabolite profiling of polyphenols in Vaccinium berries and determination of their chemopreventive properties, J. Pharm. Biomed. Anal. 89 (2014) 257-267.

[5] J.A. Kennedy, A.L. Waterhouse, Analysis of pigmented high molecular mass grape phenolics using ion-pair, normal-phase high performance liquid chromatography, J. Chromatogr. A 866 (2000) 25-35.

[6] P. Alberts, M.A. Stander, A. de Villiers, Advanced ultra high pressure liquid chromatography-tandem mass spectrometric methods for the screening of red wine anthocyanins and derived pigments, J. Chromatogr. A 1235 (2012) 92-102.

[7] A. de Villiers, G. Vanhoenacker, P. Majek, P. Sandra, Determination of anthocyanins in wine by direct injection liquid chromatography-diode array detection-mass spectrometry and classification of wines using discriminant analysis, J. Chromatogr. A 1054 (2004) 195-204.

[8] X. Wu, R.L. Prior, Systematic identification and characterization of anthocyanins by HPLC-ESI-MS/MS in common foods in the United States: fruits and berries, J. Agric. Food Chem. 53 (2005) 2589-2599.

[9] A. de Villiers, D. Cabooter, F. Lynen, G. Desmet, P. Sandra, High performance liquid chromatography analysis of wine anthocyanins revisited: effect of particle size and temperature, J. Chromatogr. A 1216 (2009) 3270-3279.

[10] A. de Villiers, D. Cabooter, F. Lynen, G. Desmet, P. Sandra, High-efficiency high performance liquid chromatographic analysis of red wine anthocyanins, J. Chromatogr. A 1218 (2011) 4660-4670

[11] G. Mazza, E. Minitiati, Anthocyanins in Fruits, Vegetables, and Grains, CRC Press Boca Raton, Florida, 1993.

[12] D.R. Stoll, J.D. Cohen, P.W. Carr, Fast, comprehensive online two-dimensiona high performance liquid chromatography through the use of high temperature ultra-fast gradient elution reversed-phase liquid chromatography, J. Chromatogr. A 1122 (2006) 123-137.

[13] P. Dugo, T. Kumm, M.L. Crupi, A. Controneo, L. Mondello, Comprehensive two-dimensional liquid chromatography combined with mass spectrometric detection in the an analyses of triacylglycerol in natural lipidic matrixes, J. Chromatogr. A 1112 (2006) 269-275.

[14] P.J. Schoenmakers, G. Vivo-Truyols, W.M.C. Decrop, A protocol for designing comprehensive two-dimensional liquid chromatography separation systems, J. Chromatogr. A 1120 (2006) 282-290.

[15] P. Dugo, O. Favoino, R. Luppino, G. Dugo, L. Mondello, Comprehensive two-dimensional normal-phase (adsorption)-reversed-phase liquid chromatography, Anal. Chem. 76 (2004) 2525-2530.

[16] F. Erni, R.W. Frei, Two-dimensional column liquid chromatographic technique for resolution of complex matrices, J. Chromatogr. 147 (1978) 561-569.

[17] P. Jandera, Column selectivity for two-dimensional liquid chromatography, J Sep. Sci. 29 (2006) 1763-1783.

[18] K.M. Kalili, A. de Villiers, Recent developments in the HPLC separation of phenolic compounds, J. Sep. Sci. 34 (2011) 845-876.

[19] K.M. Kalili, A. de Villiers, Off-line comprehensive 2-dimensional hydrophilic interaction $\times$ reversed phase liquid chromatography analysis of procyanidins, J. Chromatogr. A 1216 (2009) 6274-6284.

[20] K.M. Kalili, A. de Villiers, Off-line comprehensive two dimensional hydrophilic interaction $\times$ reversed-phase liquid chromatographic analysis of green tea phenolics, J. Sep. Sci. 33 (2010) 853-863.

[21] T. Beelders, K.M. Kalili, E. Joubert, D. de Beer, A. de Villiers, Comprehensive two dimensional liquid chromatographic analysis of rooibos (Aspalathus linearis) phenolics, J. Sep. Sci. 35 (2012) 1808-1820.

[22] C.M. Willemse, M.A. Stander, A. de Villiers, Hydrophilic interaction chromatographic analysis of anthocyanins, J. Chromatogr. A 1319 (2013) 127-140.

[23] M. Gilar, J. Fridrich, M.R. Schure, A. Jaworski, Comparison of orthogonality estimation methods for the two-dimensional separations of peptides, Anal. Chem. 84 (2012) 8722-8732.

[24] K.M. Kalili, D. Cabooter, G. Desmet, A. de Villiers, Kinetic optimisation of the reversed phase liquid chromatographic separation of proanthocyanidins on sub-2 $\mu \mathrm{m}$ and superficially porous phases, J. Chromatogr. A 1236 (2012) 63-76.

[25] K.M. Kalili, A. de Villiers, Systematic optimisation and evaluation of on-line, off-line and stop-flow comprehensive hydrophilic interaction chromatography $\times$ reversed phase liquid chromatographic analysis of procyanidin. Part I. Theoretical considerations, J. Chromatogr. A 1289 (2013) 58-68.

[26] Z. Liang, K. Li, X. Wang, Y. Ke, Y. Jin, X. Liang, Combination of off-line two-dimensional hydrophilic interaction liquid chromatography for polar fractions and two-dimensional hydrophilic interaction liquid chromatography $\times$ reversed-phase liquid chromatography for medium-polar fraction in a traditional Chinese medicine, J. Chromatogr. A 1224 (2012) 61-69.

[27] K.M. Kalili, A. de Villiers, Systematic optimisation and evaluation of on-line off-line and stop-flow comprehensive hydrophilic interaction chromatography $\times$ reversed phase liquid chromatographic analysis of procyanidins. Part II. Application to cocoa procyanidins, J. Chromatogr. A 1289 (2013) 69-79.

[28] P. Dugo, F. Cacciola, T. Kumm, G. Dugo, L. Mondello, Comprehensive multidimensional liquid chromatography: theory and applications, J. Chromatogr. A 1184 (2008) 353-368

[29] P. Jandera, T. Hájek, M. Stanková, K. Vynuchalová, P. Cesla, Optimization of comprehensive two-dimensional gradient chromatography coupling in-line hydrophilic interaction and reversed phase liquid chromatography, J. Chromatogr. A 1268 (2012) 91-101. 
[30] X. Wang, W. Li, H.T. Rasmussen, Orthogonal method development using hydrophilic interaction chromatography and reversed-phase high performance liquid chromatography for the determination of pharmaceuticals and impurities, J. Chromatogr. A 1083 (2005) 58-62.

[31] B. Abad-García, L.A. Berrueta, S. Garmón-Lobato, B. Gallo, F. Vicente, A general analytical strategy for the characterisation of phenolic compounds in fruit juices by high-performance liquid chromatography with diode array detection coupled to electrospray ionization and triple quadrupole mass spectrometry, J. Chromatogr. A 1216 (2009) 5398-5415.

[32] X. Wu, R.L. Prior, Identification and characterisation of anthocyanins by high performance liquid chromatography-electrospray ioization-tandem mass spectrometry in common foods in the United States: vegetables, nuts, and grains, J. Agric. Food Chem. 53 (2005) 3101-3113.

[33] G. Lanzarini, L. Morselli, The anthocyanins of red cabbage, Indus. Conserv. 49 (1974) 16-20

[34] J.C. Giddings, Maximum number of components resolvable by gel filtration and other elution chromatographic methods, Anal. Chem. 39 (1967) 1027-1028.

[35] J.C. Giddings, Unified Separation Science, John Wiley \& Sons, Inc., New York, 1991.

[36] J.C. Giddings, Two-dimensional separations: concept and compromise, Anal. Chem. 56 (1984) 1258A-1270A.

[37] J.C. Giddings, Concepts and comparison in multidimensional separations, J. High Res. Chromatogr. 10 (1987) 319-323

[38] U.D. Neue, Theory of peak capacity in gradient elution, J. Chromatogr. A 1079 (2005) 153-161.

[39] J.S. Barnes, H.P. Nguyen, S. Shen, K.A. Schug, General method for extraction of blueberry anthocyanins and identification using high performance liquid chromatography-electrospray ionization-ion traptime of flight-mass spectrometry, J. Chromatogr. A 1216 (2009) 4728-4735.

[40] G.J. McDougal, S. Fyffe, P. Dobson, S. Stewart, Anthocyanins from red cabbagestability to stimulated gasstrointestinal digestion, Phytochemistry 68 (2007) 1285-1294.

[41] K. Ikeda, H. Kikazaki, M. Nakamura, N. Nakatani, Structure of two acylated anthocyanins from red cabbage (Brassica oleracea), Chem. Express. 2 (1987) 563-566.

[42] E. Idaka, H. Yamakita, T. Ogawa, T. Kondo, M. Yamamoto, T. Goto, Structure of three diacylated anthocyanins isolated from red cabbage, Brassica oleracea, Chem. Lett. 6 (1987) 1213-1216.
[43] E. Idaka, K. Suzuki, H. Yaakita, T. Ogawa, T. Kondo, T. Goto, Structure of monoacylated anthocyanins isolated from red cabbage, Brassica oleracea, Chem. Lett. 1 (1987) 145-148.

[44] P. Arapitsas, P.J.R. Sjober, C. Turner, Characterisation of anthocyanins in red cabbage using high resolution liquid chromatography coupled with photodiode array detection and elctrospray ionization-linear ion trap mass spectrometry, Food Chem. 109 (2008) 219-226.

[45] I. Revilla, S. Perez-Magarino, M.L. Gonzalez-Sanjose, Identification of anthocyanin derivatives in grape skin extracts and red wines by liquid chromatography with diode array and mass spectrometric detection, J. Chromatogr. A 847 (1999) 83-90.

[46] T. Shimizu, T. Muroi, T. Ichi, M. Nakamura, K. Yoshihira, Analysis of red cabbage colors in commercial foods using high performance liquid chromatography with photodiode array detection-mass spectrometry, J. Food Hyg. Soc. Jpn. 38 (1997) 34-38.

[47] T. Ikegami, K. Tomomatsu, H. Takubo, K. Horie, N. Tanaka, Separation efficiencies in hydrophilic interaction chromatography, J. Chromatogr. A 1184 (2008) 474-503.

[48] P.W. Carr, J.M. Davis, S.C. Rutan, D.R. Stoll, Principles of online comprehensive multidimensional liquid chromatography, Adv. Chromatogr. 50 (2012) 139-235.

[49] X. Li, D.R. Stoll, P.W. Carr, Equation for peak capacity estimation in twodimensional liquid chromatography, Anal. Chem. 81 (2009) 845-850.

[50] Y. Leydet, R. Gavara, V. Petrov, A.M. Diniz, A.J. Parola, J.C. Lima, F. Pina, The effect of self-aggregation on the determination of the kinetic and thermodynamic constants of the network of chemical reactions in 3-glucoside anthocyanins, Phytochemistry 83 (2012) 125-135.

[51] R. Dück, H. Sonderfeld, O.J. Schmitz, A simple method for the determination of peak distribution in comprehensive two-dimensional liquid chromatography, J. Chromatogr. A 1246 (2012) 69-75.

[52] J.M. Davis, D.R. Stoll, P.W. Carr, Dependence of effective peak capacity in comprehensive two-dimensional separations on the distribution of peak capacity between the two dimensions, Anal. Chem. 80 (2008) 8122-8134.

[53] S.C. Rutan, J.M. Davis, P.W. Carr, Fractional coverage metrics based on ecological home range for calculation of the effective peak capacity in comprehensive two-dimensional separations, J. Chromatogr. A 1255 (2012) 267-276.

[54] G. Semard, V. Peulon-Agasse, A. Bruchet, J.P. Bouillon, P. Cardinaël, Convex hull: a new method to determine the separation space used and to optimize operating conditions for comprehensive twp-dimensional gas chromatography, J. Chromatogr. A 1217 (2010) 5449-5454. 


\section{SUPPORTING INFORMATION FOR THE MANUSCRIPT:}

Comprehensive two-dimensional liquid chromatographic analysis of anthocyanins

Chandré M. Willemse ${ }^{\mathrm{a}}$, Maria A. Stander ${ }^{\mathrm{b}, \mathrm{c}}$, Andreas G.J. Tredoux ${ }^{\mathrm{a}}$, André de Villiers ${ }^{\mathrm{a}}$

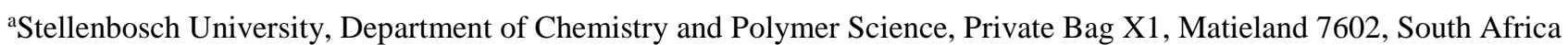

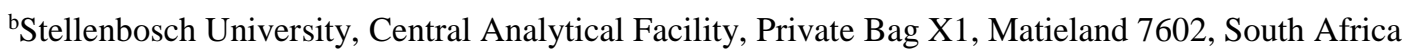

'Stellenbosch University, Department of Biochemistry, Private Bag X1, Matieland 7602, South Africa 

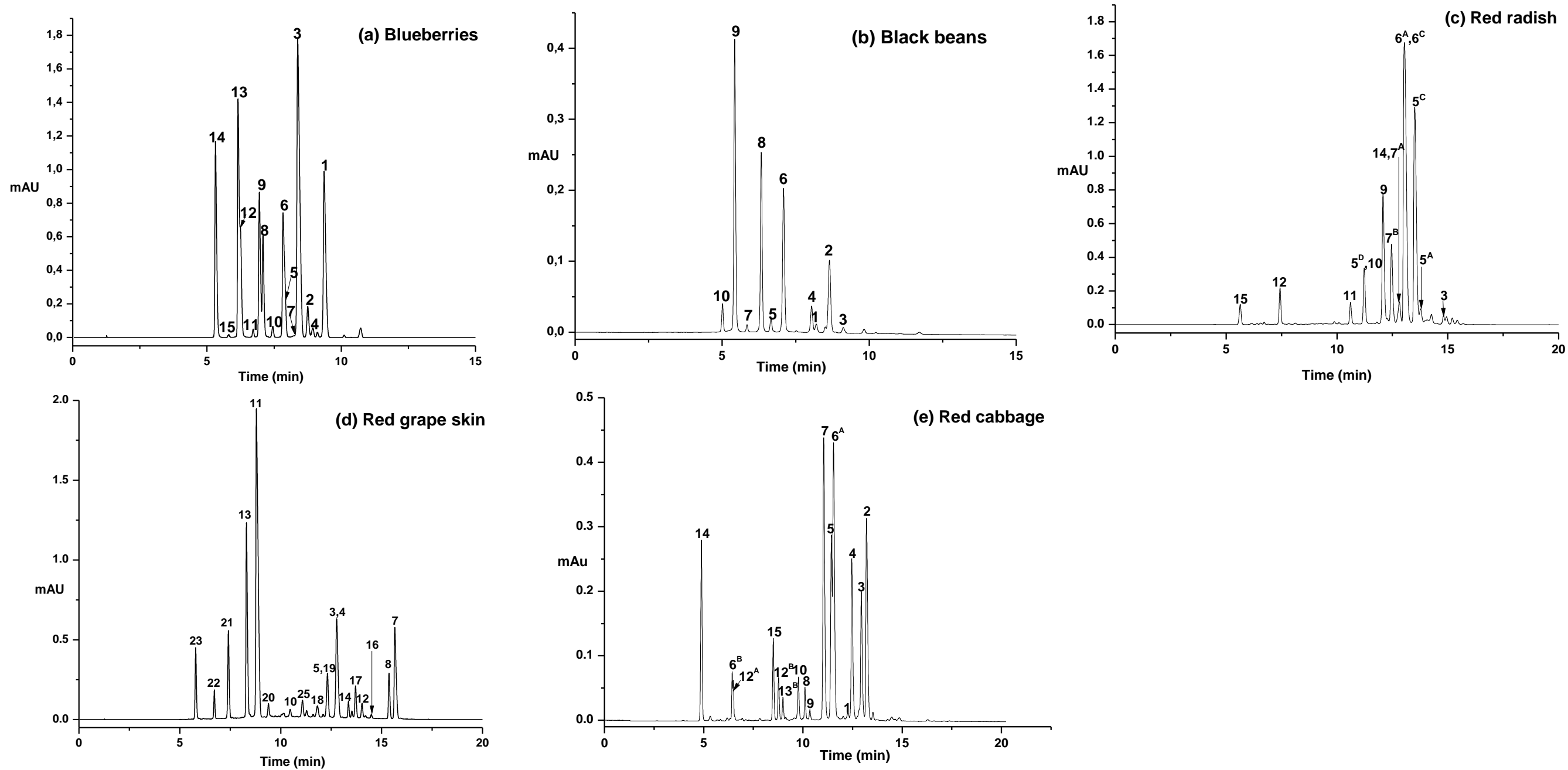

Figure S1: LC-MS-UV chromatograms obtained at $500 \mathrm{~nm}$ for the RP-LC analysis of (a) black bean, (b) blueberry, (c) red grape skin, (d) red cabbage and (e) red radish anthocyanins at $50{ }^{\circ} \mathrm{C}$ on a $\mathrm{C} 18$ column $\left(50 \times 4.6 \mathrm{~mm}\right.$ i.d., $\left.2.5 \mu \mathrm{m} d_{p}\right)$ using as mobile phase $7.5 \%$ formic in acetonitrile (solvent A)/ $7.5 \%$ formic in water (solvent B). For gradient details and further experimental conditions, refer to Section 2.4.1. Peak numbers correspond to Table 2. 
Stellenbosch University http://scholar.sun.ac.za
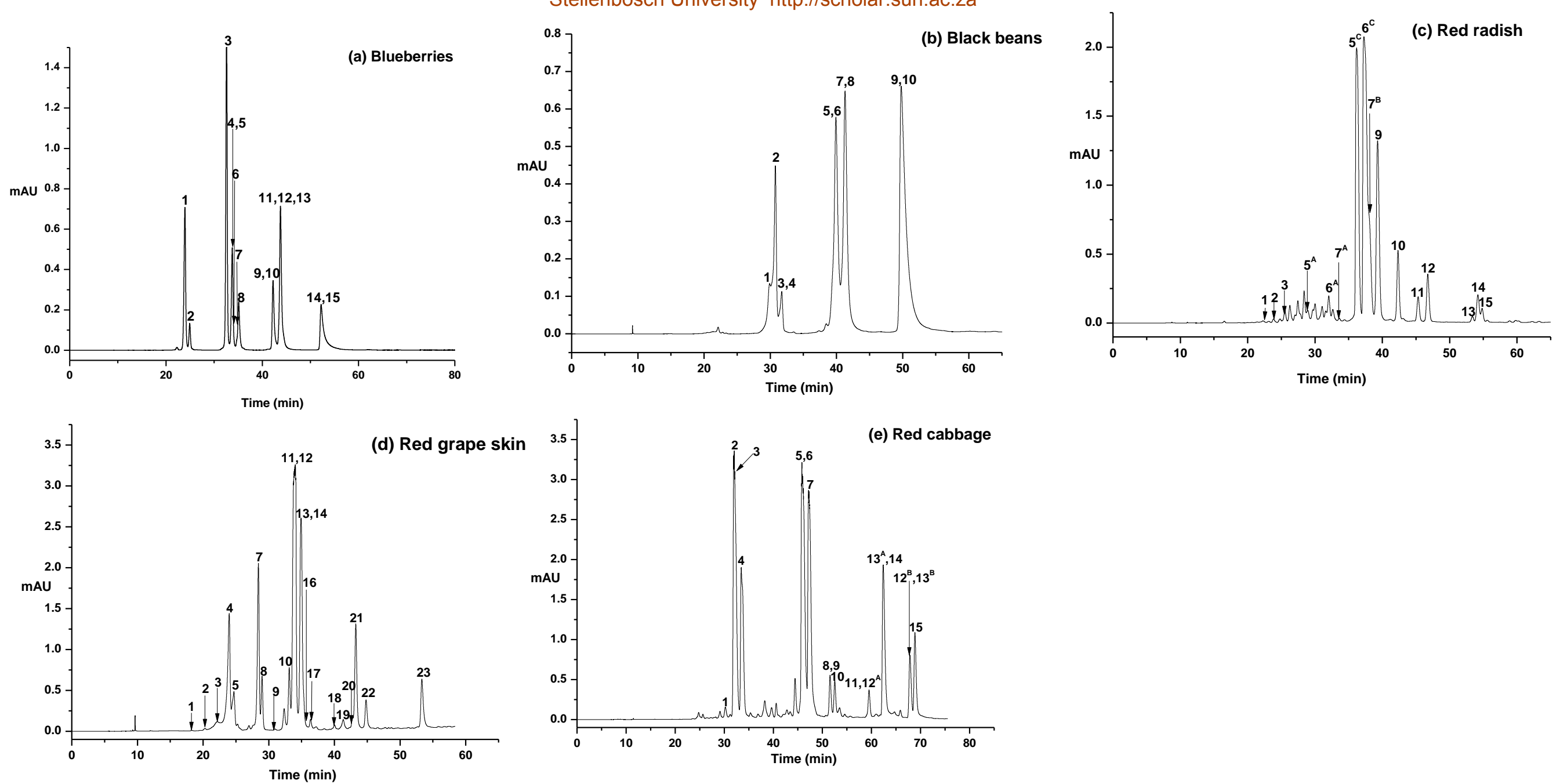

Figure S2: LC-MS-UV chromatograms obtained at $500 \mathrm{~nm}$ for the HILIC analysis of (a) black bean, (b) blueberry, (c) red grape skin, (d) red cabbage and (e) red radish anthocyanins at $50{ }^{\circ} \mathrm{C}$ on an Amide column $\left(150 \times 4.6 \mathrm{~mm}\right.$ i.d., $\left.2.5 \mu \mathrm{m} d_{p}\right)$ using as mobile phase $0.4 \%$ TFA in acetonitrile (solvent A)/ $0.4 \%$ TFA in water (solvent B). For gradient details and further experimental conditions, refer to Section 2.4.1. Peak numbers correspond to Table 2. 
(a) Blueberries

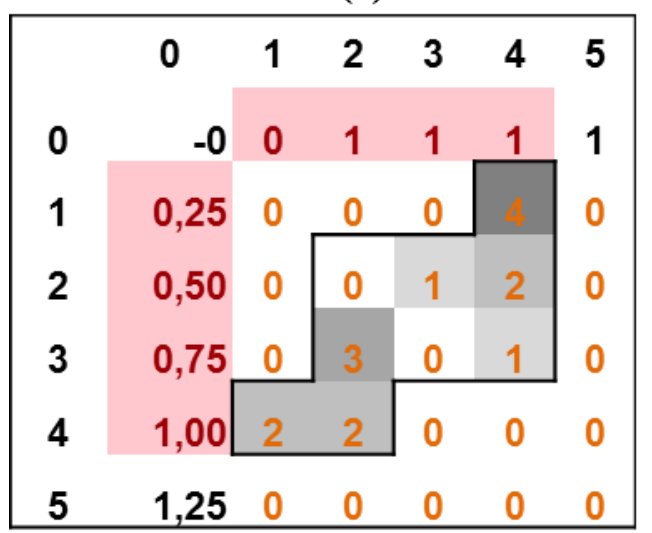

(d) Red grape skins

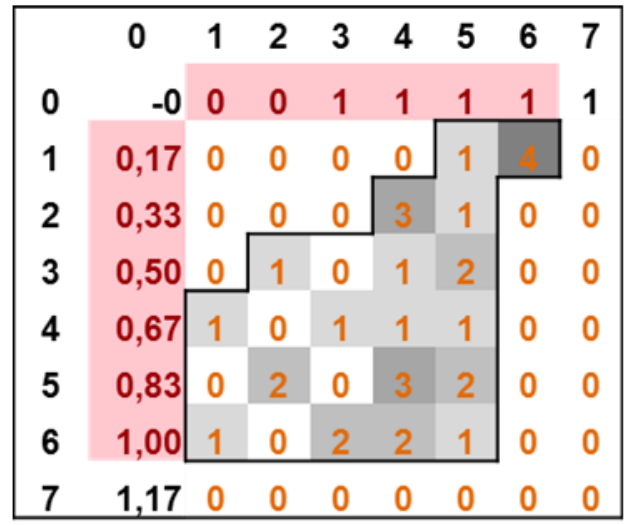

(b) Black beans

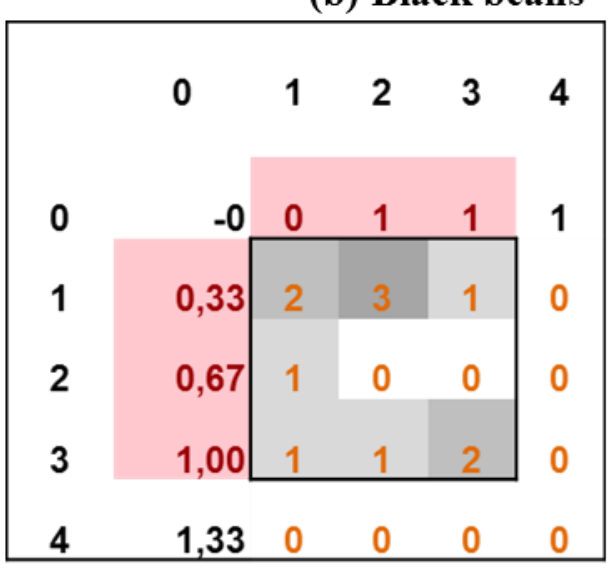

(e) Red cabbage

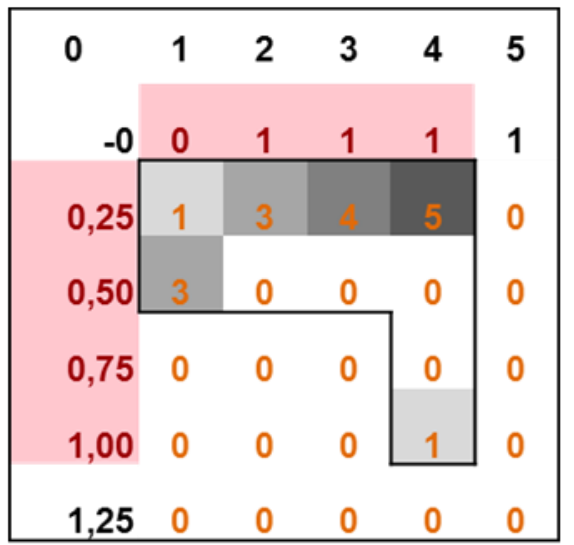

(c) Red radish

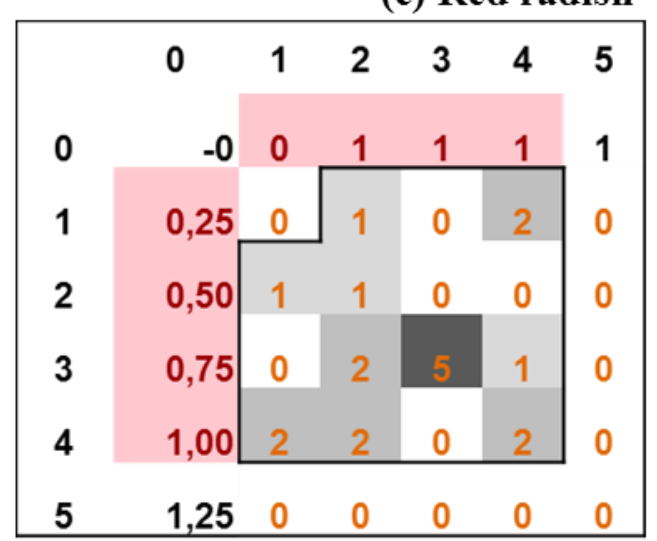

$\begin{array}{lllllll}5 & 1,25 & 0 & 0 & 0 & 0 & 0\end{array}$

Figure S3: Surface coverage plots obtained using the Stoll method [23] for (a) blueberry, (b) black bean, (c) red radish, (d) red grape skin and (e) red cabbage anthocyanins. Data are summarised in Table 3. 

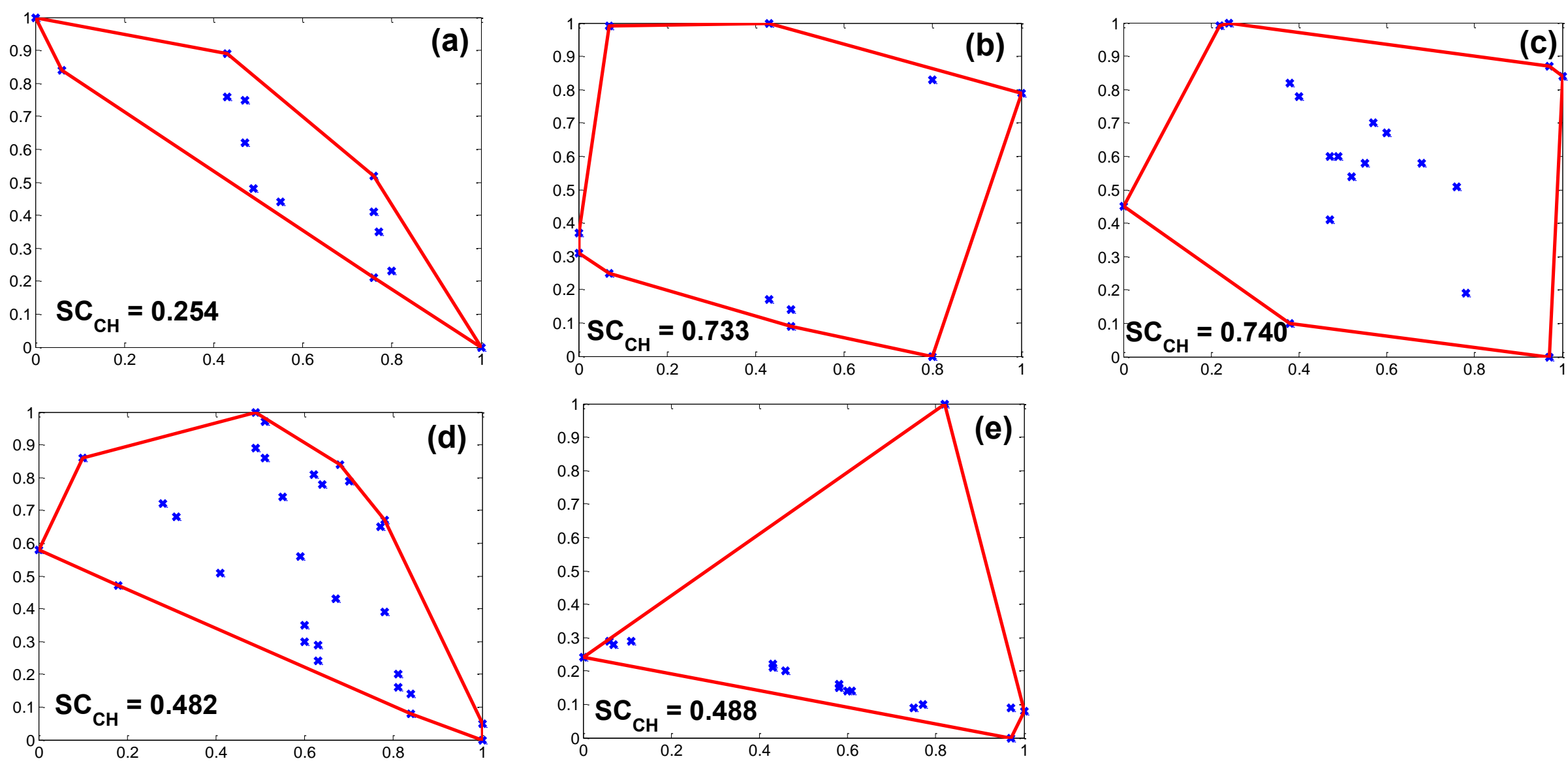

Figure S4: Surface coverage plots obtained using the convex hull method [53] for (a) blueberry, (b) black bean, (c) red radish, (d) red grape skin and (e) red cabbage anthocyanins. Data are summarised in Table 3. 


\section{Addendum C}

\section{Comprehensive two-dimensional HILIC $\times$ RP-LC-UV-MS analysis of anthocyanins and derived pigments in wine}

C.M. Willemse, M.A. Stander, J. Vestner, A.G.J. Tredoux, A. de Villiers, (submitted to Anal. Chem.)

Author contributions: C.M.W. performed all experiments, did initial data analysis and wrote the first draft of the paper. J. Vestner assisted with preparation of figures in Matlab and edited the manuscript. M.A.S. and A.G.J.T. edited the manuscript. A.d.V. assisted with MS data analysis and edited the manuscript. 


\section{Comprehensive two-dimensional HILIC $\times$ RP-LC-UV-MS analysis of anthocyanins and derived pigments in red wine}

Chandré M. Willemse ${ }^{1}$, Maria A. Stander ${ }^{2,3}$, Jochen Vestner ${ }^{4}$, Andreas G.J. Tredoux ${ }^{1}$, André de Villiers ${ }^{*}$

${ }^{1}$ Stellenbosch University, Department of Chemistry and Polymer Science, Private Bag X1, Matieland 7602, South Africa

${ }^{2}$ Stellenbosch University, Central Analytical Facility, Private Bag X1, Matieland 7602, South Africa

${ }^{3}$ Stellenbosch University, Department of Biochemistry, Private Bag X1, Matieland 7602, South Africa

${ }^{4}$ Center of Analytical Chemistry and Microbiology, Department of Microbiology and Biochemistry, Hochshule Geisenheim, Germany

*Corresponding author. Tel.: +27 21808 3351; fax +27 218083360.

E-mail address: ajdevill@sun.ac.za (A. de Villiers). 


\begin{abstract}
The chemical alteration of anthocyanins represents one of the most important transformations involved in red wine ageing. Accurate analysis of the these derived pigments, as required to study the evolution of anthocyanins and tannins during ageing, is however hampered by their extreme structural diversity, low levels, and the fact that many of these compounds have identical mass spectral characteristics. In this context, chromatographic separation is critical. In this contribution, the application of on-line hydrophilic interaction chromatography (HILIC) $\times$ reversed phase LC (RP-LC) separation coupled to high resolution MS is described for the detailed characterization of anthocyanins and their derived pigments in aged red wine. A systematic approach was followed for the optimization of HILIC $\times$ RP-LC separation parameters using a capillary LC system in the first dimension and an ultra-high pressure LC system in the second dimension to ensure maximum sensitivity and performance. Ninety four anthocyanin-derived pigments were tentatively identified in one- and six year old Pinotage wines using accurate mass and fragmentation information obtained on a quadrupole-time-offlight (Q-TOF) MS instrument. On-line HILIC $\times$ RP-LC-MS was found to offer high resolution separation due to the combination of two different separation modes, while the structured elution order observed improved certainty in compound identification. This approach therefore shows promise for the detailed elucidation of the chemical alteration of anthocyanins during wine ageing.
\end{abstract}

Key words: Comprehensive two-dimensional liquid chromatography $(L C \times L C)$, anthocyanins, derived pigments, HILIC $\times R P-L C$, wine, mass spectrometry $(M S)$ 


\section{Introduction}

Anthocyanins (anthocyanidin-glycosides) are influential constituents of red grapes and wine that play an important role in the aesthetic perception and quality and also serve as markers for wine authentication. Red grape varieties are characterized by a relatively complex mixture of anthocyanins, primarily consisting of 5 anthocyanidin-glucosides and-diglucosides and their acylated, coumaroylated and caffeoylated derivatives ${ }^{1,2}$. During the production and ageing of red wine, these grape-derived anthocyanins undergo a number of chemical transformations that play an important role in the properties of the end product. Levels of grape-derived anthocyanins rapidly decrease as they react with other wine components, leading to the formation of more stable products that are responsible for the evolution of wine color as well as sensory properties (Figure S1). Extensive research utilizing model solutions as well as wine samples in combination with MS has enabled elucidation of the most important reaction pathways. These include the $\operatorname{direct}^{3}$ and acetaldehyde-mediated ${ }^{4}$ condensation with flavanols and proanthocyanidins, the formation of anthocyanin dimers and higher oligomers ${ }^{5,6}$, and the formation of diverse pyranoanthocyanins ${ }^{3}$ through reaction of anthocyanins with acetaldehyde $^{7}$, pyruvic acid ${ }^{8,9}$, cinnamic acids ${ }^{10,11}$, acetoacetic acid ${ }^{12}$ and procyanidins in the presence of acetaldehyde ${ }^{13}$. As a result of these reactions, the complexity of the pigmented fraction of wines increases significantly during ageing. This, together with the fact that many of the formed products are present at trace levels and are themselves precursors to further reactions, is responsible for significant challenges in the accurate analysis of these compounds.

The most commonly used method for the analysis of red wine anthocyanins is reversed-phase (RP) LC, mostly coupled to diode array detection (DAD) and/or MS or tandem mass spectrometry (MS/MS). RP-LC-DAD allows for selective detection of anthocyanins at $500 \mathrm{~nm}$, whereas MS provides increased sensitivity and structural information. MS/MS is particularly beneficial for structural elucidation and compound identification ${ }^{14}$. However, RP-LCMS(/MS) also suffers from several limitations. The RP-LC separation of anthocyanins displays very low optimal flow rates as a result of on-column inter-conversion between flavylium cationic and carbinol pseudobasic forms of anthocyanins ${ }^{15,16}$, resulting in long analysis times or poor performance under sub-optimal conditions. Furthermore, the lack of commercially available standards and identical MS characteristics of several anthocyanin derivatives means that unambiguous identification of compounds is not possible in the absence of their chromatographic resolution. 
The applicability of hydrophilic interaction chromatography (HILIC) as an alternative separation mode has recently been demonstrated for the analysis of diverse anthocyanins ${ }^{17}$. This mode of separation showed clear separation of anthocyanins according to degree of glycosylation and acylation, although isomeric compounds were not resolved. The combination of HILIC and RP-LC in comprehensive two-dimensional liquid chromatography (LC $\times$ LC) has been shown to offer an exceptionally powerful approach for the analysis of complex phenolic fractions ${ }^{18-23}$. We have recently demonstrated how off-line HILIC $\times$ RP-LC analysis provides a promising approach for complex anthocyanins mixtures, including grape skin extracts ${ }^{22}$. In this contribution, the development and optimization of an on-line HILIC $\times$ RP-LC method suitable for the analysis of pigments in aged wine is reported. The optimized method was hyphenated to high resolution time-of-flight (TOF) MS detection to allow tentative identification of a wide range of the target analytes.

\section{Experimental}

\subsection{Reagents and materials}

Pinotage wine samples of 2013 and 2008 vintages were kindly donated by W.J. du Toit (Department of Enology and Viticulture, Stellenbosch University). The wines were produced using standard winemaking procedures at the Welgevallen Experimental cellar (DEV, SU), using grapes from the same vineyard and blueberries were purchased from the local supermarket. HPLC grade acetonitrile, methanol and trifluoroacetic acid (TFA) were purchased from Sigma-Aldrich (Aston Manor, South-Africa) and formic acid from Merck (Modderfontein, South-Africa). Deionised water was prepared using a Milli-Q water purification system (Millipore, Milford, MA, USA) and all mobile phases were filtered through $0.45 \mu \mathrm{m}$ HLVP membrane filters (Millipore) and degassed in an ultrasonic bath. The OASIS HLB solid phase extraction (SPE) cartridges $(20 \mathrm{~mL}, 1 \mathrm{~g})$ were from Waters (Milford, MA, USA).

\subsection{Sample preparation}

Blueberries were extracted in the same manner as previously reported ${ }^{17}$. Wine samples $(100$ $\mathrm{mL})$ were loaded onto a preconditioned $(6 \mathrm{~mL}$ methanol and $6 \mathrm{~mL}$ water acidified with $0.1 \%$ 
formic acid) SPE cartridge. The cartridge was rinsed with $2 \times 10 \mathrm{~mL}$ acidified water and eluted with $5 \mathrm{~mL}$ methanol/formic acid $(95 / 5(v / v))$. All anthocyanin containing methanolic fractions were evaporated to dryness and re-dissolved in $0.5 \mathrm{~mL}$ methanol/formic acid $(95 / 5(v / v))$ prior to HPLC analysis.

\subsection{Instrumentation and chromatographic conditions}

A Waters capillary LC (CapLC $\left.{ }^{\circledR}\right)$ system controlled by MassLynx v.4.1 software (Waters) was used in the first dimension. HILIC separation was performed on an Acquity UPLC BEH Amide column $(150 \times 1 \mathrm{~mm}$ i.d., $1.7 \mu \mathrm{m}$ particles) with a UPLC pre-column filter (Waters). The mobile phases consisted of (A) $0.4 \%$ TFA in acetonitrile and (B) $0.4 \%$ TFA in water. The gradient was as follows: 12-35\% B (0-315 min), 35-100\% B (315-324 min), 100-12\% B (324$369 \mathrm{~min}$ ). The flow rate was $1.0 \mu \mathrm{L} / \mathrm{min}$ and the column was thermostatted to $50{ }^{\circ} \mathrm{C}$. Injection volumes of 2 and $3 \mu \mathrm{L}$ were used for extracts of the 2013 and 2008 wine samples, respectively.

In the second dimension RP-LC separations were performed on a Phenomenex Kinetex C18 column $(50 \times 2.1 \mathrm{~mm}$ i.d., $1.3 \mu \mathrm{m}$ particles $)$ equipped with a security guard column (Phenomenex, Torrace, USA). Analyses were performed on an Acquity UPLC system equipped with a PDA detector controlled by Empower software (Waters). The binary mobile phases consisted of $7.5 \%(v / v)$ formic acid in water $(\mathrm{A})$ and $7.5 \%(v / v)$ formic acid in acetonitrile (B). The gradient used was as follows: $1 \% \mathrm{~B}$ (0-0.04 min), 1-13.5\% B (0.04-0.44 $\min ), 13.5-23.5 \%$ B (0.44-0.86 min), 23.5-28.5 \% B (0.86-1.0 min), $28.5 \%$ B isocratic (1.0$1.16 \mathrm{~min}$ ), before returning to initial conditions at $1.26 \mathrm{~min}$. The total analysis time including re-equilibration was $2 \mathrm{~min}$. The column temperature was set to $60^{\circ} \mathrm{C}$ and the flow rate was 860 $\mu \mathrm{L} / \mathrm{min}$.

MS and $\mathrm{MS}^{\mathrm{E}}$ data were acquired on a Waters Synapt G2 quadrupole time-of-fight (Q-TOF) instrument operated independently from a separate computer equipped with MassLynx v.4.1 software. Electrospray ionization was performed in positive mode with scan ranges of 200$2000 \mathrm{amu}$ and 40-2000 amu for MS and $\mathrm{MS}^{\mathrm{E}}$ acquisition, respectively. All other conditions were identical to those reported previously ${ }^{17}$, except that $\mathrm{MS}^{\mathrm{E}}$ acquisition was performed using a collision energy ramp of $20-60 \mathrm{~V}$. The instrument was calibrated using sodium formate solution and leucine encephalin was used as lock mass for accurate mass determination. 
For HILIC $\times$ RP-LC separations the Cap-LC and UPLC instruments were interfaced through a 10-port 2-position high pressure switching valve (1000 bar maximum pressure, $150 \mu \mathrm{m}$ bore, Valco Instruments Co. Inc., Houston Texas, USA) equipped with two $5 \mu \mathrm{L}$ loops and configured in a symmetrical manner ${ }^{24}$ (Figure S2). Switching of the valve occurred every 2 min via the UPLC external events, which resulted in a first dimension sampling time of 2 min and a second dimension injection volume of $2 \mu \mathrm{L}$ (i.e., $1 \mu \mathrm{L} / \mathrm{min} \times 2 \mathrm{~min}$ ). The RP-LC effluent was split $\sim$ 1:1 after the PDA detector to provide a flow of $\sim 430 \mu \mathrm{L} / \mathrm{min}$ to the MS.

\subsubsection{Data handling}

All optimisation and evaluation calculations were performed using Microsoft Excel. MS data analysis and identification of compounds was performed using Masslynx v.4.1. UV data for individual 2-minute second dimension analyses were exported as .csv files and used to create a matrix in Excel. Data were baseline corrected by subtracting a blank analysis and contour plots were generated using Matlab 7.14 (The Mathworks, Natick, MA, USA). MS data were acquired as a single data file for each analysis; the data were exported as .cdf files and imported into Matlab to create contour plots.

\section{Results and Discussion}

\subsection{Method optimization}

Previous work by our group has demonstrated the advantages of off-line HILIC $\times$ RP-LC for anthocyanin analysis ${ }^{22}$ : the combination of these orthogonal separation modes provides much higher resolving power, while group-type separation of different classes of anthocyanins faciliate compound identification and comparison between samples. The off-line methodology does however present several disadvantages, primarily in terms of very long analysis times (up to 55 hours) and increased risk of sample alteration during fraction collection and transfer. For the HILIC $\times$ RP-LC-MS analysis of wine pigments, therefore, an on-line methodology was developed to reduce the total analysis time (and data file size).

On-line LC $\times$ LC involves the transfer of fractions from the first dimension column directly to the second dimension column via a switching valve. Several practical implications follow from this mode of operation. First of all, the speed of the second dimension separation is critical, 
since in the time alloted for the collection of each fraction (the sampling time, ${ }^{1} t_{s}$ ), the previous fraction should be analysed and the column re-equilibrated:

$$
{ }^{1} t_{s}={ }^{2} t_{c}={ }^{2} t_{g}+{ }^{2} t_{\text {re-equil }}
$$

equation 1

Where ${ }^{2} t_{c}$ is the second dimension cycle time, which is the sum of the gradient and reequilibration times. ${ }^{1} t_{s}$ directly determines the degree of under-sampling of first-dimension peaks, while ${ }^{2} t_{c}$ determines the second dimension (and overall) peak capacity as well as the total analysis time ${ }^{25}$. Furthermore, in the absence of flow splitting, the product of the first dimension volumetric flow $\left({ }^{1} F\right)$ and the sampling time is equal to the injection volume per fraction in the second dimension, ${ }^{2} V_{\text {inj }}$ :

$$
{ }^{2} V_{i n j}={ }^{1} F \times{ }^{1} t_{s}
$$

equation 2

This is an important consideration in on-line HILIC $\times$ RP-LC, since HILIC mobile phases are strong injection solvents in RP-LC and therefore ${ }^{2} V_{i n j}$ should be minimised. In order to establish the optimal experimental conditions for on-line HILIC $\times$ RP-LC, each of these considerations was taken into account in a similar manner as outlined reported previously ${ }^{25,26}$ and briefly outlined below.

For the first dimension HILIC separation, a $1 \mathrm{~mm}$ i.d. amide column was used. A capillary LC system was employed in this dimension, since it has been shown that the optimal flow rate for anthocyanins on this column was $\sim 6 \mu \mathrm{L} / \min ^{17}$. Various first dimension gradient times $\left({ }^{1} t_{g}\right.$, 168-451 min) and flow rates ( ${ }^{1} F, 1-10 \mu \mathrm{L} / \mathrm{min}$ ) were evaluated; for each set of conditions the peak capacity was determined according to equation $3^{27}$ :

$$
n_{c, 1 D}=1+\frac{t_{g}}{1 / n \sum_{1}^{n} w_{b}}
$$

equation 3

where $t_{g}$ is the gradient time and $w_{b}$ is the peak width at baseline, averaged for $n$ peaks. In the second dimension a Kinetex $50 \times 2.1 \mathrm{~mm}$, i.d., $1.3 \mu \mathrm{m} d_{p} \mathrm{C} 18$ column was used for maximum performance for very short analysis times at a flow rate of $0.86 \mathrm{~mL} / \mathrm{min}$ to minimize reequilibration times. A column temperature of $60^{\circ} \mathrm{C}$ was used to minimize the detrimental effect of anthocyanin secondary equilibria on chromatographic performance ${ }^{15,16}$. Several gradient times (1-10 min) were evaluated on this column, and the peak capacities obtained using equation 3 were fit to the empirical relationship derived by Fairchild and co-workers ${ }^{28}$. This 
equation was used to determine the second dimension peak capacity for any cycle time in method optimization. Because the complexity of the wine extracts precluded accurate measurement of peak widths in both 1-dimensional analyses, a blueberry extract, which contains a simpler anthocyanin profile, was used to determine peak capacities in both dimensions for method optimization.

Using these two sets of peak capacity values, the practical two-dimension peak capacity could be determined for any combination of conditions by correcting for both finite orthogonality and first dimension under-sampling:

$$
n *_{c, 2 D}=\frac{{ }^{1} n_{c} \times{ }^{2} n_{c} \times f_{c}}{\beta}
$$

\section{equation 4}

where $\beta$ is a measure of under-sampling of first dimension peaks ${ }^{29}$ and $f_{\mathrm{c}}$ is the fractional surface coverage of the available 2-dimensional space ${ }^{30}$. Values for $f_{\mathrm{c}}$ were determined using the 'convex hull' method ${ }^{31}$ and normalized retention data for the compounds identified in the wine samples (refer to section 3.2, Figure S5)

Plots of $n_{c, 2 D}^{*}$ as a function of ${ }^{1} t_{g}$ and ${ }^{2} t_{c}$ for various ${ }^{1} F$ are presented in Figure S3. Most of these curves illustrate a characteristic maximum for $n_{c, 2 D}^{*}$, which is a consequence of the compromise between second dimension peak capacity (higher ${ }^{2} t_{c}$ and ${ }^{2} n_{c}$ ) and less undersampling (lower ${ }^{2} t_{c}$ and ${ }^{1} t_{s}$ ). For the lower flow rates and longer first dimension gradient times, the maximum performance is reached only at very long ${ }^{2} t_{c}$ times (> $10 \mathrm{~min}$, Figure S3A and B). Comparing different first dimension flow rates and gradient times, it is evident that higher two-dimensional peak capacities are obtained at lower first dimension flow rates and longer gradient times. This is because the optimal sampling time increases with first dimension peak width, resulting in higher second dimension and therefore overall peak capacities.

Related to these findings is the effect of first dimension flow and sampling time on the injection volume in the second dimension. Since this value is limited by the requirement to avoid injection band broadening (experimentally determined to be $2 \mu \mathrm{L}$ for the columns and phases used here), splitting of the first dimension flow is required if optimal conditions in both dimensions are to be used ${ }^{25,32}$. The drawback of this is of course a loss in sensitivity. Figure 1 
summarises the relationship between the corrected 2-dimensional peak capacity, ${ }^{2} t_{c}$ and the required split ratios for various first dimension flow rates.

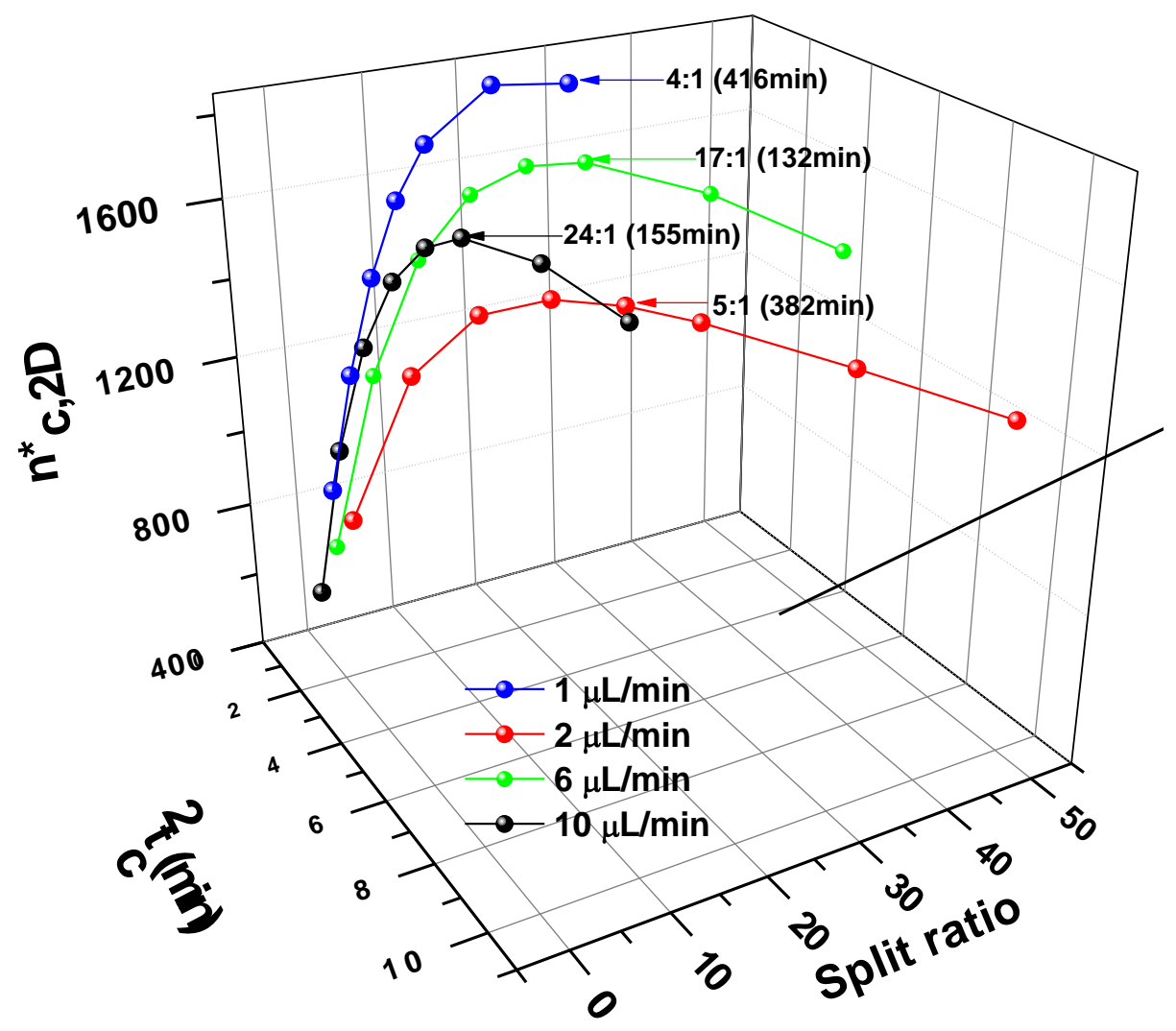

Figure 1: Plot of corrected practical 2-dimensional peak capacity $\left(n_{c, 2 D}^{*}\right)$ as a function ofsecond dimension cycle time $\left({ }^{2} t_{c}\right)$ at various first dimension flow rates. The split ratios and total analysis times required for selected points are indicated.

From Figure 1 it is evident that lower first dimension flow rates generally provide higher peak capacities, although this comes at the cost of longer overall analysis times (Figure S4). Furthermore, for a given first dimension flow rate, an increase in ${ }^{2} t_{c}$ implies a concomitant increase in the split ratio. For example, for a first dimension flow rate of $10 \mu \mathrm{L} / \mathrm{min}$ and ${ }^{2} t_{c}=$ 8 , a split ratio of 39:1 is required, whereas at $1 \mu \mathrm{L} / \mathrm{min}$ for the same ${ }^{2} t_{c}$ a split ratio of $3: 1$ is required. Furthermore, the split ratio required at the optimal second dimension cycle time decreases with the first dimension flow (indicated in Figure 1). 
Due to the low levels of anthocyanins and derived pigments in especially aged red wine, sensitivity was of paramount importance in the current study. For this reason, a low first dimension flow rate $(1 \mu \mathrm{L} / \mathrm{min})$ was selected to minimize the split ratio. For this flow rate, the optimal sampling time is $\sim 10 \mathrm{~min}$, with a requisite split ratio of $4: 1$. Initial experiments under these conditions showed insufficient sensitivity, and therefore we selected to reduce the cycle time to $2 \mathrm{~min}$ and work under splitless conditions. Under these conditions, practical two dimensional peak capacity is sacrificed for increased sensitivity (due to shorter second dimension analyses): $n_{c, 2 D}^{*}=889$ compared to $n_{c, 2 D}^{*}=2173$ at ${ }^{2} t_{c}=10 \mathrm{~min}$, split $4: 1$, indicated in Figure 1.

A summary of the performance of the HILIC $\times \mathrm{RP}-\mathrm{LC}$ separation is presented in Table $\mathrm{S} 1$. The value for the surface coverage used here was the average value for both wine samples (Figure S5), while peak capacities in both dimensions were obtained for the blueberry extract. The gain in performance offered by on-line LC separation is evident. Under the conditions used here, the effect of first-dimension under-sampling is virtually negligible, although the performance of the second dimension separation is sacrificed by the use of short cycle times to avoid flow splitting. Comparison of these performance parameters with off-line HILIC $\times$ RP-LC separation of anthocyanins ${ }^{22}$ confirms that off-line operation affords much higher peak capacities due to the first and second dimension separations being carried out independently. However, this increased performance comes at a cost of exorbitant total analyses times for off-line separations.

In the case of on-line HILIC $\times$ RP-LC, corrected 2-D peak capacities are $3.1 \times$ higher than have been reported even for highly optimized RP-LC separation of anthocyanins ${ }^{16}$. This gain in performance is especially relevant in light of the fact that many pigments in red wine cannot be distinguished by MS, as will be outlined in the next section. It is also worth noting that the reproducibility of the on-line HILIC $\times$ RP-LC method was good, with retention time variations of \pm 1 modulation period in the first dimension and $\pm 0.03 \mathrm{~min}$ in the second dimension, respectively.

\subsection{On-line HILIC $\times R P-L C-M S$ analysis of red wine pigments}

Contour plots obtained at $500 \mathrm{~nm}$ for the on-line HILIC $\times$ RP-LC-UV-MS analysis of the 2013 and 2008 Pinotage wines using the established conditions are shown in Figures 2 and 3. From 
these figures, both the complexity of wine pigments and the improved resolution offered by the combination of the complementary separation mechanisms of HILIC and RP-LC is evident. Many of the compounds detected co-elute in either one of the dimensions, and the effective 2dimensional separation space is well utilized under the experimental conditions used.

While UV detection at $500 \mathrm{~nm}$ has the advantage of allowing selective detection of pigmented molecules, hyphenation of HILIC $\times$ RP-LC with Q-TOF MS detection proved indispensible in allowing the identification of compounds based on accurate mass data, fragmentation information obtained from simultaneous $\mathrm{MS}^{\mathrm{E}}$ acquisition and comparison of relative retention times in both HILIC and RP-LC dimensions with previous literature reports ${ }^{14,16,33,34}$. On the basis of MS data, a total of 94 compounds were identified in both wine samples using relative retention times and accurate mass measurements. A summary of all compounds identified is presented in Table 1; these are discussed by class below.

Anthocyanidin-glucosides: The predominant colored species present in the young 2013 Pinotage wine are grape-derived anthocyanins, largely comprising anthocyanidin monoglucosides and their corresponding acetyl-, $p$-coumaroyl-, and caffeoyl derivatives. Malvidin3-glucoside (4) was the major pigment in both wines. Acylation of anthocyanins generally decreases HILIC retention, with the acetylated derivatives eluting slightly later than the corresponding coumaroylated derivatives ${ }^{17,22}$. Malvidin-3-caffeoyl-glucoside (15) was the sole caffeoylated derivative detected, only in the 2013 wine. Grape-derived anthocyanins are not stable in wine and their levels decrease significantly during maturation, as is evident from the much lower levels of compounds 1-15 in the 2008 wine.

Anthocyanidin-di-glucosides: Low levels of anthocyanidin-di-glucosides (malvidin-3,5diglucoside (18), malvidin-3-coumaroylglucoside-5-glucoside (19) and malvidin-3acetylglucoside-5-glucoside (20)) were tentatively identified in the 2013 Pinotage based on accurate mass and relative retention times ${ }^{16,35}$. These compounds eluted before their corresponding anthocyanidin monoglucosides in RP-LC and were highly retained in HILIC.

Oligomeric anthocyanins: The direct condensation of anthocyanins results in several oligomeric anthocyanins, four of which were tentatively identified in this sample using accurate mass data ${ }^{5,6,36}$. These include the dimers malvidin-glucoside-malvidin-glucoside- (21), malvidin-glucoside-malvidin-acetyl-glucoside (23) and malvidin-glucoside-malvidincoumaroyl-glucoside (24). The oligomeric anthocyanins are characterized by very broad peaks, which may be due to inter-conversion reactions of anthocyanins exacerbated by the fast second 
dimension cycle time ${ }^{15,16}$. Similar to the free anthocyanins, levels of the diglucosides and oligomeric anthocyanins decrease rapidly with age. As a consequence, these compounds were not detected in the 2008 Pinotage. 
Table 1: Mass spectral and retention data obtained for HILICXRP-LC-UV-MS analysis of anthocyanin and derived pigments in 2008 and 2013 Pinotage wines.

\begin{tabular}{|c|c|c|c|c|c|c|c|c|c|}
\hline $\begin{array}{c}\text { Peak } \\
\text { No. }\end{array}$ & $\begin{array}{c}\text { HILIC }^{\mathrm{a}} \\
t_{\mathrm{R}} \\
(\mathrm{min}) \\
\end{array}$ & $\begin{array}{c}\text { HILIC }^{\mathrm{b}} \\
t_{\mathrm{R}} \\
(\mathrm{min}) \\
\end{array}$ & $\begin{array}{c}\text { RP-LC } \\
t_{\mathrm{R}} \\
(\mathrm{min})\end{array}$ & $\begin{array}{c}\mathrm{RP}-\mathrm{LC} \\
t_{\mathrm{R}} \\
(\mathrm{min})\end{array}$ & Identity & $\begin{array}{c}\text { Molecular } \\
\text { Formula } \\
{[\mathrm{M}]^{+}}\end{array}$ & $\begin{array}{c}\text { Accurate } \\
\text { mass }^{c} \\
(m / z)\end{array}$ & $\begin{array}{c}\text { Error } \\
(\mathrm{ppm})^{\mathrm{d}}\end{array}$ & $M S^{\mathrm{E}}$ fragments \\
\hline & & & & & Anthocyanidin-glucosides & & & & \\
\hline 1 & 260.1 & 258.3 & 0.51 & 0.50 & Delphinidin-3-glucoside & $\mathrm{C}_{21} \mathrm{H}_{21} \mathrm{O}_{12}$ & 465.1035 & 0.2 & 303.05 \\
\hline 2 & 218.3 & 218.8 & 0.60 & 0.59 & Petunidin-3-glucoside & $\mathrm{C}_{22} \mathrm{H}_{23} \mathrm{O}_{12}$ & 479.1186 & -0.4 & $317.06,302.04,274.80,244.79$ \\
\hline \multirow[t]{2}{*}{3} & 186.2 & 185.0 & 0.67 & 0.66 & Peonidin-3-glucoside & $\mathrm{C}_{22} \mathrm{H}_{23} \mathrm{O}_{11}$ & 463.1244 & 0.4 & $301.07,286.04,258.05,229.04$ \\
\hline & & & & & & & & & $217.80,202.06$ \\
\hline \multirow[t]{2}{*}{4} & 184.3 & 183.0 & 0.72 & 0.69 & Malvidin-3-glucoside & $\mathrm{C}_{23} \mathrm{H}_{25} \mathrm{O}_{12}$ & 493.1348 & 0.2 & $331.08,315.05,287.05,270.05$ \\
\hline & & & & & & & & & 242.05 \\
\hline 5 & 200.3 & n.d. ${ }^{\mathrm{e}}$ & 0.73 & n.d. & Delphinidin-3-acetylglucoside & $\mathrm{C}_{23} \mathrm{H}_{23} \mathrm{O}_{13}$ & 507.1143 & 0.4 & 303.05 \\
\hline 6 & 168.3 & n.d. & 0.73 & n.d. & Cyanidin-3-acetylglucoside & $\mathrm{C}_{23} \mathrm{H}_{23} \mathrm{O}_{12}$ & 491.1286 & 9.6 & 287.05 \\
\hline 7 & 168.3 & n.d. & 0.84 & n.d. & Petunidin-3-acetylglucoside & $\mathrm{C}_{24} \mathrm{H}_{25} \mathrm{O}_{13}$ & 521.1295 & 0 & $317.06,302.04,274.04,244.79$ \\
\hline 8 & 144.3 & n.d. & 0.92 & n.d. & Peonidin-3-acetylglucoside & $\mathrm{C}_{24} \mathrm{H}_{25} \mathrm{O}_{12}$ & 505.1354 & 0.8 & $301.00,286.04,258.05,229.04$ \\
\hline \multirow{3}{*}{9} & & & & & & & & & 202.05 \\
\hline & 142.4 & 141.3 & 0.96 & 0.94 & Malvidin-3-acetylglucoside & $\mathrm{C}_{25} \mathrm{H}_{27} \mathrm{O}_{13}$ & 535.1456 & 0.4 & $331.08,315.04,299.05,287.05$, \\
\hline & & & & & & & & & $270.05,242.05$ \\
\hline
\end{tabular}




\begin{tabular}{|c|c|c|c|c|c|c|c|c|c|}
\hline 10 & 204.5 & 205.3 & 0.92 & 0.90 & Delphinidin-3-coumaroyl-glucoside & $\mathrm{C}_{30} \mathrm{H}_{27} \mathrm{O}_{14}$ & 611.1399 & -0.2 & $303.05,274.80$ \\
\hline 11 & 176.5 & n.d. & 0.97 & n.d. & Cyanidin-3-coumaroyl-glucoside & $\mathrm{C}_{30} \mathrm{H}_{27} \mathrm{O}_{13}$ & 595.1454 & 0.2 & 287.05 \\
\hline 12 & 172.5 & 171.3 & 1.01 & 1.01 & Petunidin-3-coumaroyl-glucoside & $\mathrm{C}_{31} \mathrm{H}_{29} \mathrm{O}_{14}$ & 625.1557 & 0 & $317.06,302.04,274.04,246.79$ \\
\hline 13 & 150.5 & 151.4 & 1.10 & 1.09 & Peonidin-3-coumaroyl-glucoside & $\mathrm{C}_{31} \mathrm{H}_{29} \mathrm{O}_{13}$ & 609.1598 & -1.0 & $301.07,286.04,258.05$ \\
\hline \multirow[t]{2}{*}{14} & 148.1 & 145.5 & 1.13 & 1.13 & Malvidin-3-coumaroyl-glucoside & $\mathrm{C}_{32} \mathrm{H}_{31} \mathrm{O}_{14}$ & 639.1712 & -0.2 & $331.08,315.05,299.05,287.05$, \\
\hline & & & & & & & & & $270.05,242.05$ \\
\hline \multirow[t]{2}{*}{15} & 166.5 & n.d. & 1.01 & n.d. & Malvidin-3-caffeoyl-glucoside & $\mathrm{C}_{32} \mathrm{H}_{31} \mathrm{O}_{15}$ & 655.1665 & 0.2 & 331.08 \\
\hline & & & & & $\underline{\text { Anthocyanidin-di-glucosides }}$ & & & & \\
\hline 16 & 282.1 & n.d. & 0.65 & n.d. & Delphinidin-diglucoside $(3,5$ - or 3,7$)$ & $\mathrm{C}_{27} \mathrm{H}_{31} \mathrm{O}_{17}$ & 627.1472 & -8.9 & 303.04 \\
\hline 17 & 246.3 & n.d. & 0.71 & n.d. & Malvidin-3,7-diglucoside & $\mathrm{C}_{29} \mathrm{H}_{35} \mathrm{O}_{17}$ & 655.1807 & -6.7 & 331.08 \\
\hline 18 & 266.2 & n.d. & 0.64 & n.d. & Malvidin-3,5-diglucoside & $\mathrm{C}_{29} \mathrm{H}_{35} \mathrm{O}_{17}$ & 655.1917 & 4.3 & 331.08 \\
\hline 19 & 226.6 & n.d. & 0.99 & n.d. & Malvidin-3-coumaroylglucoside-5-glucoside & $\mathrm{C}_{38} \mathrm{H}_{41} \mathrm{O}_{19}$ & 801.2216 & -0.2 & 331.08 \\
\hline \multirow[t]{2}{*}{20} & 224.4 & n.d. & 0.76 & n.d. & Malvidin-3-acetylglucoside-5-glucoside & $\mathrm{C}_{31} \mathrm{H}_{37} \mathrm{O}_{18}$ & 697.9181 & 0.1 & 331.08 \\
\hline & & & & & Oligomeric anthocyanins & & & & \\
\hline 21 & 282.4 & n.d. & 0.89 & n.d. & Malvidin-glucoside dimer & $\mathrm{C}_{46} \mathrm{H}_{49} \mathrm{O}_{24}$ & 985.2612 & -0.2 & $823.20,644.13,331.08$ \\
\hline 22 & 264.4 & n.d. & 0.82 & n.d. & Malvidin-glucoside dimer & $\mathrm{C}_{46} \mathrm{H}_{49} \mathrm{O}_{24}$ & 985.2792 & 17.8 & $823.19,331.07$ \\
\hline
\end{tabular}




\begin{tabular}{|c|c|c|c|c|c|c|c|c|c|}
\hline 23 & 226.6 & n.d. & 0.98 & n.d. & $\begin{array}{l}\text { Malvidin-glucoside-malvidin-acetylglucoside } \\
\text { dimer }\end{array}$ & $\mathrm{C}_{48} \mathrm{H}_{51} \mathrm{O}_{25}$ & 1027.2719 & 0 & $823.21,331.08$ \\
\hline 24 & 232.7 & n.d. & 1.06 & n.d. & $\begin{array}{l}\text { Malvidin-glucoside-malvidin-coumaroyl- } \\
\text { glucoside dimer }\end{array}$ & $\mathrm{C}_{55} \mathrm{H}_{55} \mathrm{O}_{26}$ & 1131.3027 & 4.5 & $969.25,823.21,661.15,331.08$ \\
\hline 25 & 218.3 & n.d. & 0.7 & n.d. & $\begin{array}{l}\text { Malvidin-malvidin-glucoside dimer } \\
\text { Direct anthocyanin-tannin adducts }\end{array}$ & $\mathrm{C}_{41} \mathrm{H}_{39} \mathrm{O}_{19}$ & 823.2136 & 2.1 & 331.08 \\
\hline 26 & 270.1 & 268.2 & 0.50 & 0.50 & Catechin-malvidin-glucoside (F-A type) & $\mathrm{C}_{38} \mathrm{H}_{37} \mathrm{O}_{18}$ & 781.1985 & 0.5 & $619.14,331.08$ \\
\hline 27 & n.d. & 270.1 & n.d. & 0.46 & $\begin{array}{l}\text { (Epi)catechin-(C4-C6)-malvidin-glucoside } \\
\text { (F-A type) }\end{array}$ & $\mathrm{C}_{38} \mathrm{H}_{37} \mathrm{O}_{18}$ & 781.1925 & -5.5 & n.d. \\
\hline 28 & n.d. & 270.2 & n.d. & 0.58 & Epicatechin-malvidin-glucoside (F-A type) & $\mathrm{C}_{38} \mathrm{H}_{37} \mathrm{O}_{18}$ & 781.1977 & -0.3 & $619.15,467.10,331.08$ \\
\hline 29 & n.d. & 270.2 & n.d. & 0.55 & $\begin{array}{l}\text { Malvidin-glucoside-(epi)gallocatechin (F-A } \\
\text { type) }\end{array}$ & $\mathrm{C}_{38} \mathrm{H}_{37} \mathrm{O}_{18}$ & 797.1209 & n.d. & 653.0641 \\
\hline 30 & n.d. & 214.9 & n.d. & 0.71 & $\begin{array}{l}\text { Malvidin-acetylglucoside-(epi)catechin (F-A } \\
\text { type) }\end{array}$ & $\mathrm{C}_{40} \mathrm{H}_{39} \mathrm{O}_{19}$ & 823.2107 & 2.1 & 619.15 \\
\hline 31 & 196.6 & 195.5 & 1.06 & 1.08 & $\begin{array}{l}\text { Malvidin-glucoside-di(epi)catechin (F-A } \\
\text { type) }\end{array}$ & $\mathrm{C}_{53} \mathrm{H}_{49} \mathrm{O}_{24}$ & 1069.2690 & 7.6 & n.d. \\
\hline
\end{tabular}




\begin{tabular}{|c|c|c|c|c|c|c|c|c|c|}
\hline 32 & n.d. & 268.3 & n.d. & 0.6 & $\begin{array}{l}\text { Malvidin-glucoside-(epi)catechin- } \\
\text { (epi)gallocatechin (A-F type) }\end{array}$ & $\mathrm{C}_{53} \mathrm{H}_{51} \mathrm{O}_{25}$ & 1087.2644 & -7.5 & n.d. \\
\hline 33 & 244.3 & 238.61 & 0.6 & 0.6 & $\begin{array}{l}\text { Malvidin-glucoside-di(epi)catechin (A-F } \\
\text { type) }\end{array}$ & $\mathrm{C}_{53} \mathrm{H}_{51} \mathrm{O}_{24}$ & 1071.2787 & 1.7 & n.d. \\
\hline 34 & 164.3 & n.d. & 0.82 & n.d. & $\begin{array}{l}\text { Malvidin-acetylglucoside-(epi)catechin (A-F } \\
\text { type) }\end{array}$ & $\mathrm{C}_{40} \mathrm{H}_{41} \mathrm{O}_{19}$ & 825.2252 & 1.0 & n.d. \\
\hline 35 & 190.3 & 189.0 & 0.72 & 0.71 & Malvidin-glucoside-catechin (A-F type) & $\mathrm{C}_{38} \mathrm{H}_{39} \mathrm{O}_{18}$ & 783.2079 & -5.7 & 621.15 \\
\hline 36 & 196.4 & 193.1 & 0.84 & 0.82 & Malvidin-glucoside-epicatechin (A-F type) & $\mathrm{C}_{38} \mathrm{H}_{39} \mathrm{O}_{18}$ & 783.2133 & -0.3 & $621.16,469.10$ \\
\hline 37 & n.d. & 197.0 & n.d. & 0.68 & $\begin{array}{l}\text { Malvidin-glucoside-(C4-C6)-(epi)catechin } \\
\text { (A-F type) }\end{array}$ & $\mathrm{C}_{38} \mathrm{H}_{39} \mathrm{O}_{18}$ & 783.2133 & -0.3 & 621.15 \\
\hline 38 & 226.5 & 224.9 & 0.80 & 0.83 & $\begin{array}{l}\text { Peonidin-coumaroyl-glucoside-(epi)catechin } \\
\text { (F-A type) }\end{array}$ & $\mathrm{C}_{46} \mathrm{H}_{41} \mathrm{O}_{19}$ & 897.2348 & 10.6 & n.d. \\
\hline 39 & 222.5 & 223.0 & 0.85 & 0.86 & $\begin{array}{l}\text { Malvidin-coumaroyl-glucoside-catechin (F-A } \\
\text { type) }\end{array}$ & $\mathrm{C}_{47} \mathrm{H}_{43} \mathrm{O}_{20}$ & 927.2372 & 2.4 & 619.14 \\
\hline 40 & 226.5 & n.d. & 0.87 & n.d. & $\begin{array}{l}\text { Malvidin-coumaroyl-glucoside-(epi)catechin } \\
\text { (F-A type) }\end{array}$ & $\mathrm{C}_{47} \mathrm{H}_{43} \mathrm{O}_{20}$ & 927.2339 & -0.9 & 619.14 \\
\hline
\end{tabular}

\section{Acetaldehyde-mediated tannin adducts}




\begin{tabular}{|c|c|c|c|c|c|c|c|c|c|}
\hline 41 & 198.9 & 199.5 & 1.30 & 1.23 & Malvidin-ethyl-di(epi)catechin & $\mathrm{C}_{55} \mathrm{H}_{53} \mathrm{O}_{24}$ & 1097.2914 & -1.3 & n.d. \\
\hline 42 & 248.5 & n.d. & 0.82 & n.d. & Malvidin-glucoside-ethyl-(epi)gallocatechin & $\mathrm{C}_{40} \mathrm{H}_{41} \mathrm{O}_{19}$ & 825.2242 & 0 & n.d. \\
\hline 43 & 258.5 & n.d. & 0.81 & n.d. & Malvidin-glucoside-ethyl-gallocatechin & $\mathrm{C}_{40} \mathrm{H}_{41} \mathrm{O}_{19}$ & 825.2154 & -8.8 & n.d. \\
\hline 44 & 216.8 & n.d. & 1.12 & n.d. & $\begin{array}{l}\text { Malvidin-coumaroyl-glucoside-ethyl- } \\
\text { (epi)gallocatechin }\end{array}$ & $\mathrm{C}_{49} \mathrm{O}_{47} \mathrm{O}_{21}$ & 971.2663 & 5.3 & n.d. \\
\hline 45 & 232.5 & 234.8 & 0.85 & 0.84 & $\begin{array}{l}\text { Malvidin-glucoside-(C4-C6)-ethyl- } \\
\text { (epi)catechin }\end{array}$ & $\mathrm{C}_{40} \mathrm{H}_{41} \mathrm{O}_{18}$ & 809.2312 & 1.9 & n.d. \\
\hline 46 & 238.4 & 236.8 & 0.78 & 0.78 & Petunidin-glucoside-ethyl-epicatechin & $\mathrm{C}_{39} \mathrm{H}_{39} \mathrm{O}_{18}$ & 795.2179 & 4.3 & n.d. \\
\hline 47 & 248.4 & 246.6 & 0.76 & 0.72 & Petunidin-glucoside-ethyl-catechin & $\mathrm{C}_{39} \mathrm{H}_{39} \mathrm{O}_{18}$ & 795.2176 & 4.0 & n.d. \\
\hline 48 & 220.5 & 223.0 & 0.85 & 0.87 & Peonidin-glucoside-ethyl-(epi)catechin & $\mathrm{C}_{39} \mathrm{H}_{39} \mathrm{O}_{17}$ & 779.2220 & 3.3 & n.d. \\
\hline 49 & 210.5 & n.d. & 0.89 & n.d. & Malvidin-glucoside-ethyl-epicatechin & $\mathrm{C}_{40} \mathrm{H}_{41} \mathrm{O}_{18}$ & 809.2315 & 2.2 & n.d. \\
\hline 50 & 220.5 & n.d. & 0.87 & n.d. & Malvidin-glucoside-ethyl-catechin & $\mathrm{C}_{40} \mathrm{H}_{41} \mathrm{O}_{18}$ & 809.2284 & -0.9 & 647.19 \\
\hline 51 & 182.6 & n.d. & 1.01 & n.d. & Malvidin-acetylglucoside-ethyl-catechin & $\mathrm{C}_{42} \mathrm{H}_{43} \mathrm{O}_{19}$ & 851.2388 & -1.1 & n.d. \\
\hline 52 & 188.7 & n.d. & 1.10 & n.d. & Malvidin-acetylglucoside-ethyl-epicatechin & $\mathrm{C}_{42} \mathrm{H}_{43} \mathrm{O}_{19}$ & 851.2471 & 7.2 & n.d. \\
\hline 53 & 200.8 & n.d. & 1.18 & n.d. & $\begin{array}{l}\text { Peonidin-coumaroylglucoside-ethyl- } \\
\text { (epi)catechin }\end{array}$ & $\mathrm{C}_{48} \mathrm{H}_{45} \mathrm{O}_{19}$ & 925.2524 & -3.1 & n.d. \\
\hline
\end{tabular}




\begin{tabular}{|c|c|c|c|c|c|c|c|c|c|}
\hline 54 & 190.6 & n.d. & 1.10 & n.d. & $\begin{array}{l}\text { Malvidin-coumaroyl-glucoside-ethyl- } \\
\text { (epi)catechin }\end{array}$ & $\mathrm{C}_{49} \mathrm{H}_{47} \mathrm{O}_{20}$ & 955.2663 & 0.2 & n.d. \\
\hline 55 & 196.8 & 199.5 & 1.21 & 1.21 & $\begin{array}{l}\text { Malvidin-coumaroyl-glucoside-ethyl- } \\
\text { (epi)catechin }\end{array}$ & $\mathrm{C}_{49} \mathrm{H}_{47} \mathrm{O}_{20}$ & 955.2580 & -8.1 & n.d. \\
\hline 56 & 202.7 & n.d. & 1.10 & n.d. & $\begin{array}{l}\text { Malvidin-coumaroyl-glucoside-ethyl- } \\
\text { (epi)catechin }{ }^{\mathrm{f}} \\
\text { Anthocyanin-vinylflavanol condensation } \\
\text { products }\end{array}$ & $\mathrm{C}_{49} \mathrm{H}_{47} \mathrm{O}_{20}$ & 955.2682 & 2.1 & n.d. \\
\hline 57 & 248.5 & n.d. & 0.90 & n.d. & $\begin{array}{l}\text { Malvidin-acetylglucoside-vinyl- } \\
\text { di(epi)catechin }\end{array}$ & $\mathrm{C}_{57} \mathrm{H}_{51} \mathrm{O}_{25}$ & 1135.2878 & 15.9 & n.d. \\
\hline 58 & 250.4 & 246.7 & 0.77 & 0.78 & Delphinidin-acetylglucoside-vinyl-catechin & $\mathrm{C}_{40} \mathrm{H}_{35} \mathrm{O}_{19}$ & 819.1815 & 4.2 & n.d. \\
\hline 59 & 222.6 & 217.1 & 0.94 & 0.92 & Petunidin-acetylglucoside-vinyl-catechin & $\mathrm{C}_{41} \mathrm{H}_{37} \mathrm{O}_{19}$ & 833.1886 & -4.3 & n.d. \\
\hline 60 & 194.6 & 193.3 & 1.05 & 1.02 & Peonidin-acetylglucoside-catechin & $\mathrm{C}_{41} \mathrm{H}_{37} \mathrm{O}_{18}$ & 817.1991 & 0.6 & 643.12 \\
\hline 61 & 196.6 & 193.3 & 1.02 & 1.02 & Malvidin-acetylglucoside-vinyl-catechin & $\mathrm{C}_{42} \mathrm{H}_{39} \mathrm{O}_{19}$ & 847.2087 & 0.1 & n.d. \\
\hline 62 & n.d. & 181.5 & n.d. & 1.15 & Malvidin-acetylglucoside-vinyl-(epi)catechin & $\mathrm{C}_{42} \mathrm{H}_{39} \mathrm{O}_{19}$ & 847.2104 & 1.8 & 643.14 \\
\hline 63 & 172.5 & n.d. & 0.98 & n.d. & $\begin{array}{l}\text { Malvidin-acetylglucoside-vinyl-(C4-C6)- } \\
\text { (epi)catechin }\end{array}$ & $\mathrm{C}_{42} \mathrm{H}_{39} \mathrm{O}_{19}$ & 847.2118 & 3.2 & n.d. \\
\hline
\end{tabular}




\begin{tabular}{|c|c|c|c|c|c|c|c|c|c|}
\hline 64 & 188.7 & 189.5 & 1.16 & 1.15 & $\begin{array}{l}\text { Malvidin-coumaroyl-glucoside-vinyl- } \\
\text { (epi)catechin }\end{array}$ & $\mathrm{C}_{49} \mathrm{H}_{43} \mathrm{O}_{20}$ & 951.2275 & -7.3 & 643.14 \\
\hline 65 & 233.4 & 232.1 & 0.71 & 0.68 & Malvidin-glucoside-vinyl-(epi)gallocatechin & $\mathrm{C}_{40} \mathrm{H}_{37} \mathrm{O}_{19}$ & 821.1866 & -6.3 & 659.14 \\
\hline 66 & 278.4 & 272.5 & 0.86 & 0.84 & Malvidin-glucoside-vinyl-di(epi)catechin & $\mathrm{C}_{55} \mathrm{H}_{49} \mathrm{O}_{24}$ & 1093.2726 & 11.2 & $931.20,641.13,623.58$ \\
\hline 67 & 214.7 & 213.3 & 1.05 & 1.05 & Malvidin-glucoside-vinyl-catechin & $\mathrm{C}_{40} \mathrm{H}_{37} \mathrm{O}_{18}$ & 805.1970 & -1.4 & $643.14,491.09$ \\
\hline 68 & 214.8 & 211.3 & 1.13 & 1.14 & $\begin{array}{l}\text { Malvidin-glucoside-vinyl-(epi)catechin } \\
\underline{\text { Oxovitisins }}\end{array}$ & $\mathrm{C}_{40} \mathrm{H}_{37} \mathrm{O}_{18}$ & 805.1989 & 0.5 & $643.14,491.09$ \\
\hline 69 & 166.5 & 163.3 & 0.98 & 0.99 & Pyranone-malvidin-glucoside & $\mathrm{C}_{25} \mathrm{H}_{25} \mathrm{O}_{13}$ & 533.1314 & 1.9 & 371.07 \\
\hline 70 & 142.5 & 137.4 & 1.11 & 1.09 & Pyranone-malvidin-acetylglucoside & $\mathrm{C}_{27} \mathrm{H}_{27} \mathrm{O}_{14}$ & 575.1371 & -3.0 & n.d. \\
\hline 71 & 160.7 & 153.4 & 1.23 & 1.21 & $\begin{array}{l}\text { Pyranone-malvidin-coumaroyl-glucoside } \\
\underline{\text { Anthocyanin-pyruvic acid products }}\end{array}$ & $\mathrm{C}_{34} \mathrm{H}_{31} \mathrm{O}_{15}$ & 679.1638 & -2.5 & 371.07 \\
\hline 72 & 196.3 & 197.0 & 0.77 & 0.75 & Malvidin-glucoside-pyruvic acid (Vitisin A) & $\mathrm{C}_{26} \mathrm{H}_{25} \mathrm{O}_{14}$ & 561.1258 & 1.4 & $399.06,383.04,355.03,310.05$ \\
\hline 73 & 156.2 & 157.1 & 0.78 & 0.79 & $\begin{array}{l}\text { Malvidin-acetylglucoside-pyruvic acid } \\
\text { (Acetylvitisin A) }\end{array}$ & $\mathrm{C}_{28} \mathrm{H}_{27} \mathrm{O}_{15}$ & 603.1342 & -0.8 & $399.07,383.04,355.04$ \\
\hline 74 & 162.4 & 157.3 & 0.96 & 0.94 & $\begin{array}{l}\text { Malvidin-coumaroyl-glucoside-pyruvic acid } \\
\text { (Coumaroylvitisin A) }\end{array}$ & $\mathrm{C}_{35} \mathrm{H}_{31} \mathrm{O}_{16}$ & 707.1608 & -0.4 & 399.06 \\
\hline
\end{tabular}




\begin{tabular}{|c|c|c|c|c|c|c|c|c|c|}
\hline 75 & n.d. & 199.0 & n.d. & 0.70 & Peonidin-glucoside-pyruvic acid & $\mathrm{C}_{25} \mathrm{H}_{23} \mathrm{O}_{13}$ & 531.1162 & 2.3 & 365.05 \\
\hline 76 & n.d. & 232.6 & n.d. & 0.61 & Petunidin-glucoside-pyruvic acid & $\mathrm{C}_{25} \mathrm{H}_{23} \mathrm{O}_{14}$ & 547.1139 & 5.1 & 385.05 \\
\hline \multirow[t]{2}{*}{77} & n.d. & 187.2 & n.d. & 0.83 & Petunidin-coumaroyl-glucoside-pyruvic acid & $\mathrm{C}_{34} \mathrm{H}_{29} \mathrm{O}_{16}$ & 693.1465 & 0.9 & 385.05 \\
\hline & & & & & Anthocyanin-acetaldehyde derivatives & & & & \\
\hline 78 & 158.2 & n.d. & 0.79 & n.d. & Malvidin-glucoside-acetaldehyde (Vitisin B) & $\mathrm{C}_{25} \mathrm{H}_{25} \mathrm{O}_{12}$ & 517.1389 & 4.3 & 355.08 \\
\hline \multirow[t]{2}{*}{79} & 138.5 & n.d. & 1.05 & n.d. & Malvidin-coumaroyl-glucoside-acetaldehyde & $\mathrm{C}_{34} \mathrm{H}_{31} \mathrm{O}_{14}$ & 663.1703 & -1.1 & 355.07 \\
\hline & & & & & (Acetylvitisin B) & & & & \\
\hline 80 & 170.1 & 166.9 & 0.55 & 0.56 & Delphinidin-glucoside-acetaldehyde & $\mathrm{C}_{23} \mathrm{H}_{21} \mathrm{O}_{12}$ & 489.1029 & -0.4 & n.d. \\
\hline \multirow[t]{2}{*}{81} & 156.3 & 153.2 & 0.92 & 0.9 & Delphinidin-acetylglucoside-acetaldehyde & $\mathrm{C}_{25} \mathrm{H}_{23} \mathrm{O}_{13}$ & 531.1128 & -1.1 & n.d. \\
\hline & & & & & Anthocyanin-vinylcatechol derivatives & & & & \\
\hline 82 & n.d. & 175.5 & n.d. & 1.13 & Malvidin-glucoside-vinylcatechol (Pinotin A) & $\mathrm{C}_{31} \mathrm{H}_{29} \mathrm{O}_{14}$ & 625.1581 & 2.4 & $463.10,447.07,419.08$ \\
\hline 83 & n.d. & 143.5 & n.d. & 1.2 & Malvidin-acetylglucoside-vinylcatechol & $\mathrm{C}_{33} \mathrm{H}_{31} \mathrm{O}_{15}$ & 667.1660 & -0.3 & 463.10 \\
\hline 84 & n.d. & 147.6 & n.d. & 1.26 & Malvidin-coumaroyl-glucoside-vinylcatechol & $\mathrm{C}_{40} \mathrm{H}_{35} \mathrm{O}_{16}$ & 771.1889 & -3.6 & 463.10 \\
\hline 85 & 148.6 & 147.6 & 1.2 & 1.23 & Peonidin-coumaroyl-glucoside-vinylcatechol & $\mathrm{C}_{39} \mathrm{H}_{33} \mathrm{O}_{15}$ & 741.1796 & -2.3 & n.d. \\
\hline 86 & 208.5 & 211.2 & 1.05 & 1.01 & Petunidin-glucoside-vinylcatechol & $\mathrm{C}_{30} \mathrm{H}_{27} \mathrm{O}_{14}$ & 611.1375 & -2.6 & 449.08 \\
\hline & & & & & Anthocyanin-vinylphenol derivatives & & & & \\
\hline
\end{tabular}




\begin{tabular}{|c|c|c|c|c|c|c|c|c|c|}
\hline 87 & 150.6 & 151.5 & 1.19 & 1.19 & Malvidin-glucoside-vinylphenol (Pigment A) & $\mathrm{C}_{31} \mathrm{H}_{29} \mathrm{O}_{13}$ & 609.1608 & 0 & $447.10,431.07$ \\
\hline 88 & 126.7 & 125.5 & 1.27 & 1.26 & Malvidin-acetylglucoside-vinylphenol & $\mathrm{C}_{33} \mathrm{H}_{31} \mathrm{O}_{14}$ & 651.1709 & -0.5 & 447.10 \\
\hline \multirow[t]{2}{*}{89} & 176.5 & 177.4 & 1.1 & 1.1 & Petunidin-glucoside-vinylphenol & $\mathrm{C}_{30} \mathrm{H}_{27} \mathrm{O}_{13}$ & 595.1395 & -5.7 & $433.09,417.08$ \\
\hline & & & & & Anthocyanin-vinylguaicol derivatives & & & & \\
\hline 90 & 150.7 & 147.6 & 1.22 & 1.23 & Malvidin-glucoside-vinylguaicol & $\mathrm{C}_{32} \mathrm{H}_{31} \mathrm{O}_{14}$ & 639.1734 & 2.0 & 477.11 \\
\hline 91 & n.d. & 147.6 & n.d. & 1.23 & Peonidin-glucoside-vinylguaicol & $\mathrm{C}_{31} \mathrm{H}_{29} \mathrm{O}_{13}$ & 609.1555 & -5.3 & n.d. \\
\hline 92 & n.d. & 179.5 & n.d. & 1.15 & Petunidin-glucoside-vinylguaicol & $\mathrm{C}_{31} \mathrm{H}_{29} \mathrm{O}_{14}$ & 625.1687 & 13 & 463.10 \\
\hline \multirow[t]{2}{*}{93} & n.d. & 205.3 & n.d. & 1.02 & Delphinidin-glucoside-vinylguaicol & $\mathrm{C}_{30} \mathrm{H}_{27} \mathrm{O}_{14}$ & 611.1388 & -1.3 & 449.08 \\
\hline & & & & & Anthocyanin-vinylsyringol derivatives & & & & \\
\hline 94 & n.d. & 145.6 & n.d. & 1.29 & Malvidin-glucoside-vinylsyringol & $\mathrm{C}_{33} \mathrm{H}_{33} \mathrm{O}_{15}$ & 669.1773 & -4.6 & 507.32 \\
\hline
\end{tabular}

${ }^{a}$ Retention times for the 2013 wine. ${ }^{b}$ Retention times for the 2008 wine. ${ }^{\mathrm{c}}$ Experimental accurate mass. ${ }^{\mathrm{d}}$ difference between experimental and theoretical accurate mass in parts per million (ppm). ${ }^{\mathrm{e}}$ Not detected. ${ }^{\mathrm{f}}$ identities of the isomers could not be determined based on retention and MS data. 
Comparison between Figure 2 and 3 also shows that most of the grape-derived anthocyanins identified in Figure 2 had been replaced by derived pigments in the older wine. A large number of pigments formed from the grape-derived anthocyanins during production and/or ageing were tentatively identified in both the Pinotage wines.

Anthocyanin-tannin adducts: Anthocyanin-proanthocyanin adducts are mostly formed during the early stages of wine maturation ${ }^{35,37}$. Three different classes of compounds resulting from reactions involving anthocyanins and flavanols can be distinguished. The first group comprises the direct condensation products between anthocyanins and proanthocyanidins. Depending on the relative positions of the flavanol and anthocynain moieties, flavanol-anthocyanin (F-A) and anthocyanin-flavanol (A-F) configurations can be distinguished. The former compounds result from nucleophilic attack of anthocyanins in their hydrated forms on cationic flavanols, themselves originating from cleavage of procyanidins, while the latter class are formed from nucleophilic addition of the flavanol at $\mathrm{C} 4$ of an anthocyanin in the flavylium cationic form $^{3,37-}$ 43. Several direct tannin adducts were tentatively identified, including five A-F and six F-A adducts detected mostly in the 2008 wine. The F-A adducts are more retained in HILIC and elute earlier than the A-F adducts in RP-LC.

A second class of anthocyanin-proanthocyanin adducts is formed by acetaldehyde-mediated condensation of anthocyanins and flavonols, leading to ethyl-linked adducts ${ }^{13,44,45}$. Sixteen acetaldehyde mediated condensation products were tentatively identified in the 2013 wine, only six of which were detected in the 2008 wine. This observation confirms that these compounds are progressively replaced during wine ageing.

Anthocyanin-vinylflavanol condensation products are formed through cycloaddition of vinylflavanols (produced from cleavage of larger procyanidins) and anthocyanins ${ }^{44,46-51}$. Several vinylflavanol condensation products were detected, including the vinyl(epi)catechin derivatives of malvidin-glucoside $(67,68)$, malvidin- $(61)$, peonidin- $(60)$, petunidin- $(59)$ and delphinidin-acetylglucoside (58), as well as two dimeric vinylflavanol adducts $(\mathbf{5 7}, \mathbf{6 6})$. These higher oligomers are largely separated from the majority of pigments by their higher retention in the HILIC dimension.

Several isomeric species were detected for the anthocyanin-tannin adducts. Since mass spectral data for these compounds were identical, tentative identification was based on the RP-LC 
elution orders: in the case of two isomers, the catechin-derivative elutes before the epicatechin isomer. For some compounds, three isomers were observed, with the earliest eluting compound in RP-LC assigned to the C4-C6 adducts of the catechin species ${ }^{42}$.

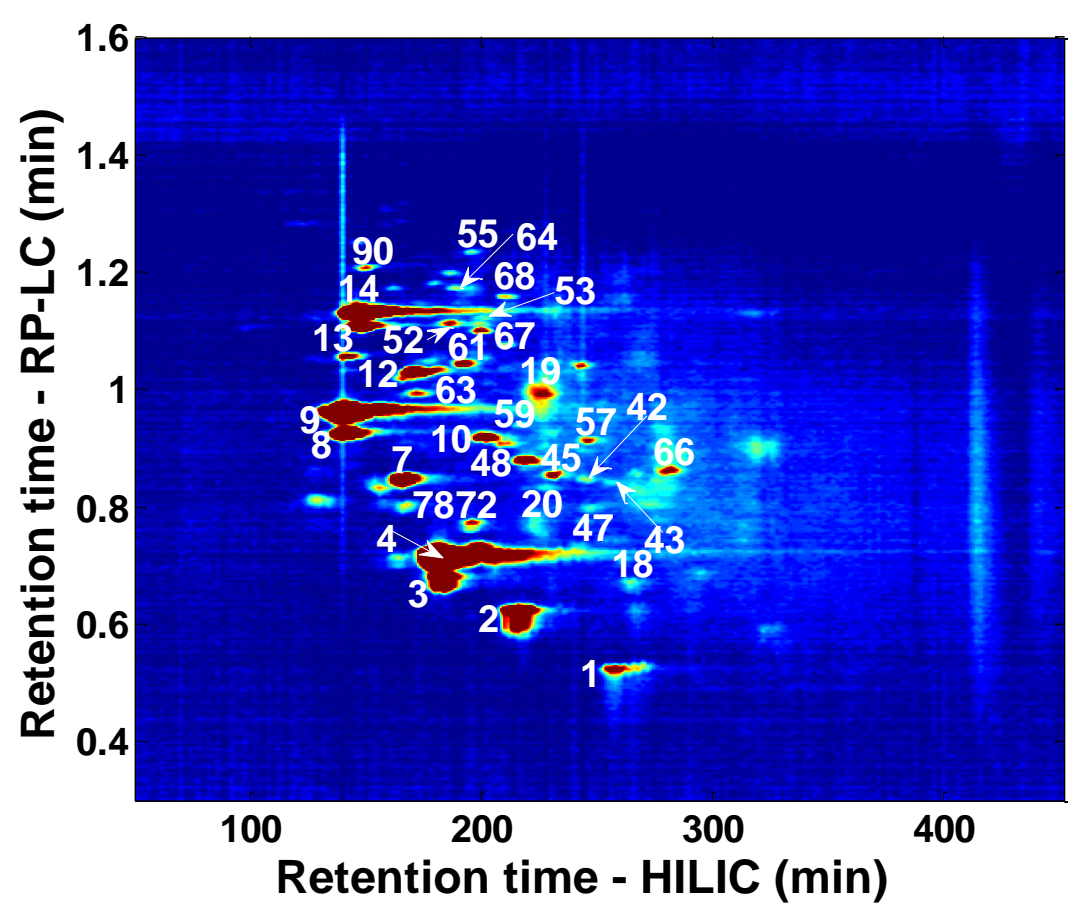

Figure 2: Contour plot for the on-line HILIC $\times$ RP-LC-UV-MS analysis of a 2013 Pinotage wine obtained at $500 \mathrm{~nm}$. For gradient details and experimental conditions, refer to Section 2.3.2. Peak numbers correspond to Table 1 and Figure 3.

Pyranoanthocyanins: Pyranoanthocyanins are a group of stabilized pigments formed via the cycloaddition of compounds containing a polarizable double bond such as pyruvic acid, acetaldehyde and vinylphenols with anthocyanins at the $\mathrm{C} 4$ position ${ }^{7,9,11,52}$. These pigments are mainly formed from grape-derived anthocyanins during fermentation of must and later during maturation and ageing of red wines ${ }^{53}$. Anthocyanin-pyruvic acid and -acetaldehyde derivatives, the so-called Vitisin A and B compounds, respectively, are usually the most abundant pyranoanthocyanins found in red wine ${ }^{7,8,54}$. Six A-type vitisin derivatives, corresponding to the pyruvic acid derivatives of malvidin- (72), peonidin-(75) and petunidinglucoside (76) and the acetyl (73) and coumaroylated derivatives $(\mathbf{7 4 , 7 7 )}$ of malvidin-3glucoside were detected in the 2008 wine; only the malvidin derivatives were observed in the 
younger wine. In contrast, four B-type vitisin derivatives were identified in the 2013 Pinotage, while only the delphinidin-glucoside (80) and delphinidin-acetylglucoside-acetaldehyde (81) derivatives were detected in the older wine.

Anthocyanin-vinylphenol condensation products are formed by reaction between cinnamic acids and anthocyanins ${ }^{11}$. Five vinylcatechol, three vinylphenol, four vinylguaicol and one vinylsyringol derivatives were identified in the Pinotage wines. Especially the -vinylcatechol and-vinylguiacol derivatives were much more prevalent in the six year old wine. Noteworthy is that Pinotin A (malvidin-glucoside-vinylcatechol, 82) was not detected in the 2013 wine, but was one of the major pigments (based on peak area) in the older Pinotage. These observations are in line with reports that levels of anthocyanin-vinyl-phenol derivatives increase with wine $\operatorname{age}^{35,55}$.

A third class of pyranoanthocyanins, the oxovitisins, are second-generation pigments formed through the nucleophilic attack of water on vitisins ${ }^{56}$. Three oxovitisins were detected (69-71); as expected, their levels were much higher in the older Pinotage wine.

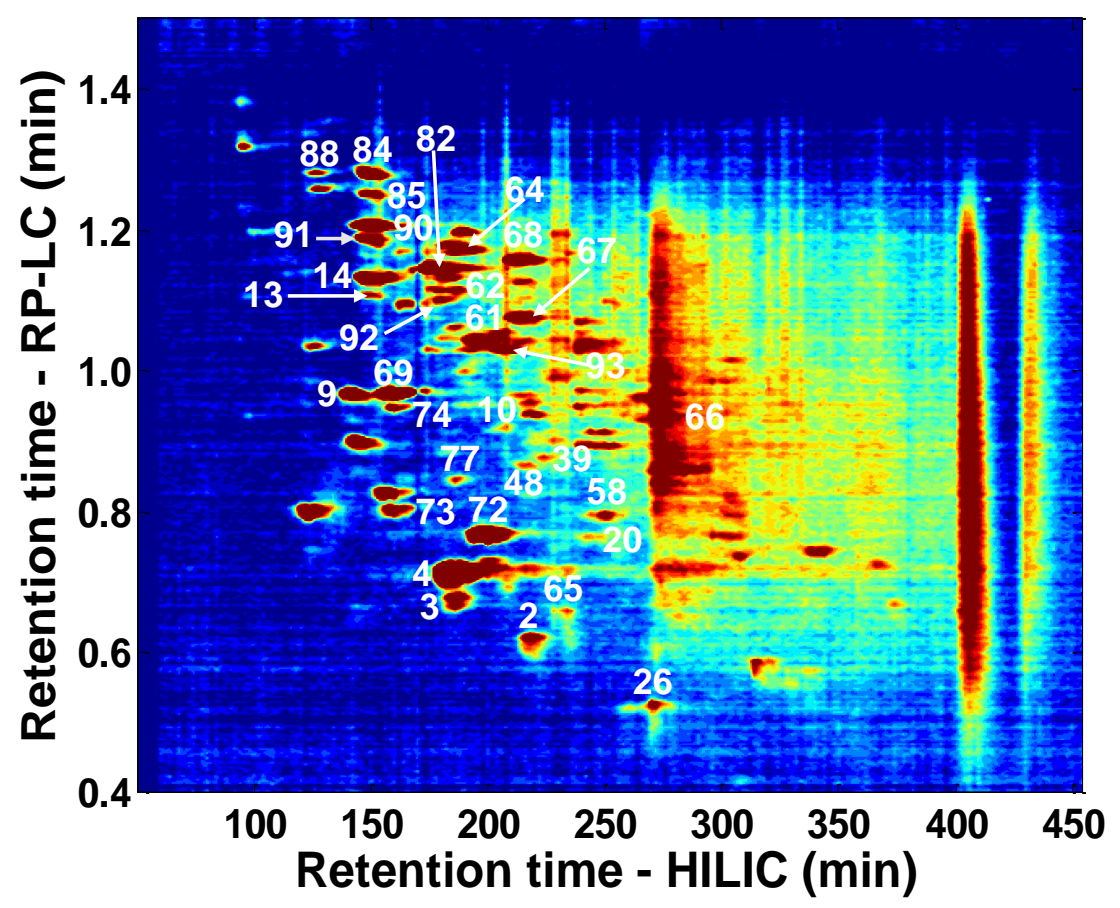

Figure 3: Contour plot for the on-line HILIC $\times$ RP-LC-UV-MS analysis of a 2008 Pinotage wine obtained at $500 \mathrm{~nm}$. For gradient details and experimental conditions, refer to Section 2.3.2. Peak numbers correspond to Table 1 and Figure 2. 


\subsection{Comparison of pigment composition between young and aged Pinotage wines based on HILIC $\times R P-L C$ contour plots}

Chromatographic retention data in two dimensions, especially in combination with mass spectral data, provides useful information for the identification of anthocyanins and their derivatives. An additional important benefit of HILIC $\times$ RP-LC separation is that the contour plots obtained facilitate the comparison of pigment profiles in young and aged wines by means of visual comparison. This is demonstrated in the retention data plots for the 2013 and 2008 Pinotage wines shown in Figure 4, where the compounds present in both samples are identified based on their presence at the same position in the two-dimensional separation space. It has previously been shown that group-type separation is observed for the HILIC $\times$ RP-LC analysis of anthocyanins ${ }^{22}$. The anthocyanidin-mono-glucosides, -diglucosides, oligomeric anthocyanins (red circles, squares and triangles, respectively), anthocyanin-tannin adducts and anthocyanin-vinylflavanol condensation products (blue squares and triangles, respectively) all appear on the right-hand side of the contour plots. These groups of compounds are highly retained in HILIC and weakly to moderately retained in RP-LC. In the bottom left of the plots the anthocyanidin-acylated derivatives (red squares), anthocyanin-tannin adducts (blue squares), -pyruvic acid derivatives and acetaldehyde derivatives (black circles and triangles) are observed; these compounds are less retained in both RP-LC and HILIC dimensions. Compounds showing high retention in RP-LC and low retention in HILIC (top left of the plots) include the anthocyanin-coumaroylated derivarives (red squares), acetaldehyde-mediated tannin adducts, vinylflavanol condensation products (blue circles and triangles), oxovitisins (black circles), -vinylcatechol and -vinylphenol derivatives (magenta circles and squares). This retention information proves invaluable as an additional criterion in compound identification: from the position of a peak in the two-dimensional separation space, information may be gained about its relative polarity with reference to known compounds. 

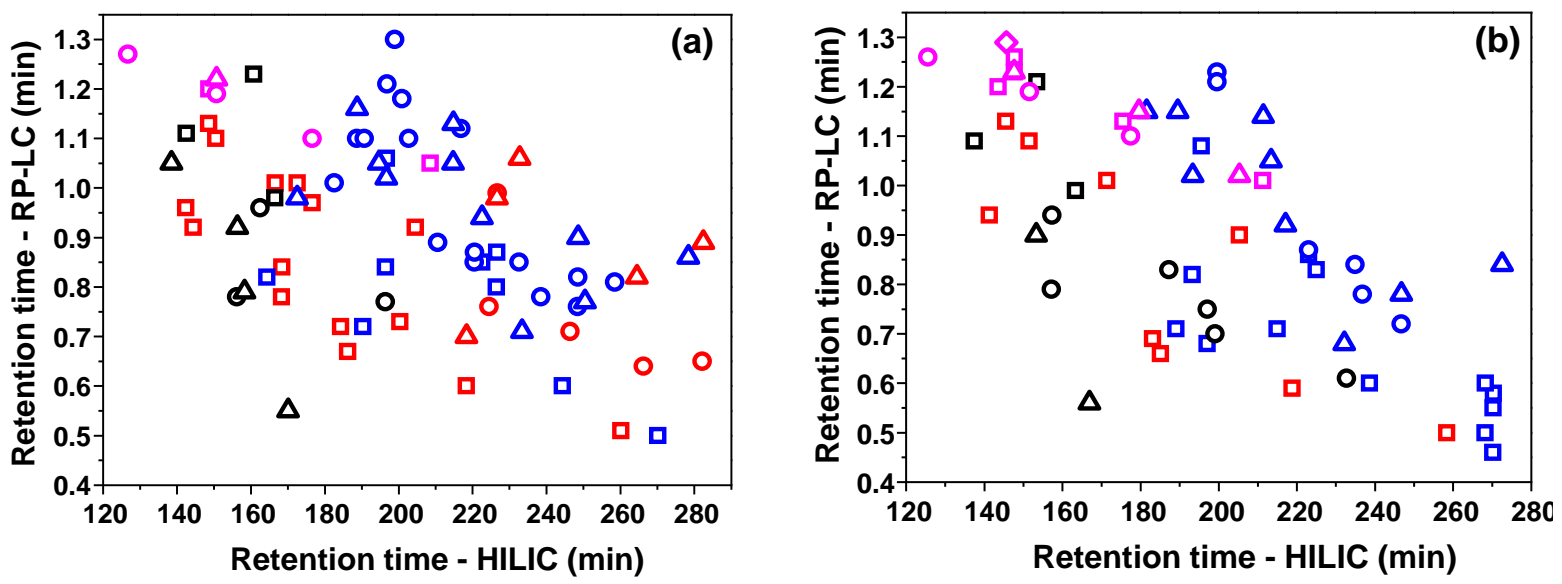

Figure 4: Retention data plot for the HILIC $\times$ RP-LC analysis of anthocyanins and derived pigments in (a) 2013 and (b) 2008 Pinotage wine. Symbols; $\square$ anthocyanidin-glucoside, $\bigcirc$ anthocyanidin-diglucoside, $\Delta$ oligomeric anthocyanins, $\square$ anthocyanin-tannin adducts, $\bigcirc$ acetaldehyde-mediated tannins, $\Delta$ vinylflavanol condensation products, $\square$ oxovitisins, $O$ anthocyanin-pyruvic acid derivatives, $\Delta$ anthocyanin-acetaldehyde derivatives, anthocyanin-catechol derivative, $\square$ anthocyanin-phenol derivative, $\Delta$ anthocyanin-guaicol derivative and $\diamond$ anthocyanin-syringol derivatives.

The plots in Figure 4 also facilitate evaluation of the evolution of pigments in Pinotage wines with age. For example, from Figure 4 the absence of anthocyanidin-diglucosides (red circles) and oligomeric anthocyanins (red triangles) in the 2008 wine sample is evident, and can be attributed to the significant decrease in concentration of the grape-derived anthocyanins during wine ageing. On the other hand, more anthocyanin-tannin adducts (blue squares), acetaldehyde-mediated tannins (blue circles) and anthocyanin-pyruvic acid derivatives (black circles) are detected in the aged wine.

\section{Conclusions}

The evolution of anthocyanins plays a critical role in red wine processing and ageing, yet elucidation of the reactions involved and their effect on the pigment composition is hampered by the complexity and low levels of the products involved. It is only recently that "second 
generation" derivatives could be identified through the application of highly selective analytical methodologies. In this contribution on-line HILIC $\times$ RP-LC-UV-MS has been shown to be a powerful method for the separation and identification of a wide range of anthocyanin derivatives found in young and aged red wines. The combination of two independent chromatographic retention mechanisms with accurate mass data and fragmentation information increases the certainty in compound identification. Improved chromatographic resolution facilitates distinguishing between compounds with identical MS characteristics, while the group-type separation obtained by HILIC $\times$ RP-LC provides an additional means of identification. UV contour plots facilitate visual comparison of pigment profiles between wines. HILIC $\times$ RP-LC-MS was therefore shown to offer a powerful method for screening of wine pigments, in addition to the potential identification of new molecules and pathways involved in their formation during wine ageing.

\section{Acknowledgements}

The authors gratefully acknowledge Sasol (grant to AdV and bursary to CW), the National Research Foundation (NRF, South Africa, Grant 81830 to AdV) and the International Foundation of Science (IFS, Sweden, Grant F/4904-1 to AdV) for financial support. W.J. du Toit is gratefully acknowledged for the kind donation of the wine samples. 


\section{References}

(1) Mazza, G.; Brouillard, R. Food Chem. 1987, 25, 207-225.

(2) Castañeda-Ovando, A.; Pacheco-Hernández, M. L.; Páez-Hernández, M. E.;

Rodríguez, J. A.; Galán-Vidal, C. A. Food Chem. 2009, 113, 859-871.

(3) Remy, S.; Fulcrand, H.; Labarbe, B.; Cheynier, V.; Moutounet, M. J. Sci. Food Agric. 2000, 80, 745-.

(4) Fulcrand, H.; Doco, T.; Es-Safi, N. E.; Cheynier, V.; Moutounet, M. J. Chromatogr. A. 1996, 752, 85-91.

(5) Alcalde-Eon, C.; Escribano-Bailon, M. T.; Santos-Buelga, C.; Rivas-Gonzalo, J. C. J. Mass Spectrom. 2007, 42, 735-748.

(6) Vidal, S.; Meudec, E.; Cheynier, V.; Skouroumounis, G.; Hayasaka, Y. J. Agric. Food Chem. 2004, 52, 7144-7151.

(7) Bakker, J.; Timberlake, C. F. J. Agric. Food Chem. 1997, 45, 35-43.

(8) Bakker, J.; Bridle, P.; Honda, T.; Kuwano, H.; Saito, N.; Terahara, N.; Timberlake, C. F. Phytochem. 1997, 44, 1375-1382.

(9) Fulcrand, H.; Benabdeljalil, C.; Rigaud, J.; Cheynier, V.; Moutounet, M. Phytochem. 1998, 47, 1401-1407.

(10) Fulcrand, H.; Cameira-dos-Santos, P. J.; Sanri-Manchado, P.; Cheynier, V.; FavreBonvin, J. J. Chem. Soc. Perkin Trans. 1996, 1, 735-739.

(11) Schwarz, M.; Wabnitz, T. C.; Winterhalter, P. J. Agric. Food Chem. 2003, 51, 36823687.

(12) He, J.; Santos-Buelga, C.; Silva, A. M. S.; Mateus, N.; de Freitas, V. J. Agric. Food Chem. 2006, 54, 9598-9603.

(13) Rivas-Gonzalo, J. C.; Bravo-Haro, S.; Santos-Buelga, C. J. Agric. Food Chem. 1995, 43, 1444-1449.

(14) Alberts, P.; Stander, M. A.; de Villiers, A. J. Chromatogr. A 2012, 1235, 92-102.

(15) de Villiers, A.; Cabooter, D.; Lynen, F.; Desmet, G.; Sandra, P. J. Chromatogr. A. 2009, 1216, 3270-3279.

(16) de Villiers, A.; Cabooter, D.; Lynen, F.; Desmet, G.; Sandra, P. J. Chromatogr. A. 2011, 1218, 4660-4670.

(17) Willemse, C. M.; Stander, M. A.; de Villiers, A. J. Chromatogr. A. 2013, 1319, $127-$ 140 . 
(18) Kalili, K. M.; de Villiers, A. J. Sep. Sci. 2011, 34, 854-876.

(19) Kalili, K. M.; de Villiers, A. J. Chromatogr. A. 2009, 1216, 6274-6284.

(20) Kalili, K. M.; de Villiers, A. J. Sep. Sci. 2010, 33, 853-863.

(21) Beelders, T.; Kalili, K. M.; Joubert, E.; de Beer, D.; de Villiers, A. J. Sep. Sci. 2012, $35,1808-1820$.

(22) Willemse, C. M.; Stander, M. A.; Tredoux, A. G. J.; de Villiers, A. J. Chromatogr. A. 2014, 1359, 189-201.

(23) Kalili, K. M.; Vestner, J.; Stander. M. A.; de Villiers, A. Anal. Chem. 2013, 85, $9107-$ 9115.

(24) van der Horst, A.; Schoenmakers, P. J. J. Chromatogr. A. 2003, 1000, 693-709.

(25) Kalili, K. M.; de Villiers, A. J. Chromatogr. A. 2013, 1289, 58-68.

(26) Kalili, K. M.; de Villiers, A. J. Chromatogr. A. 2013, 1289, 69- 79.

(27) Neue, U. D. J. Chromatogr. A. 2005, 1079, 153-161.

(28) Fairchild, J. N.; Horváth, K.; Guiochon, G. J. Chromatogr. A. 2009 1216, 1363-1371.

(29) Li, X.; Stoll, R. D.; Carr, P. W. Anal. Chem. 2009, 81, 845-850.

(30) Gilar, M.; Fridrich, J.; Schure, M. R.; Jaworski, A. Anal. Chem. 2012, 84, 8722-8732.

(31) Semard, G.; Peulon-Agasse, V.; Bruchet, A.; Bouillon, J. P.; Cardinaël, P. J.

Chromatogr. A. 2010, 1217, 5449-5454.

(32) Filqueira, M. R.; Huang, Y.; Witt, K.; Castells, C.; Carr, P. W. Anal. Chem. 2011, 83, 9531-9539.

(33) de Villiers, A.; Vanhoenacker, G.; Majek, P.; Sandra, P. J. Chromatogr. A. 2004, 1054, 195-204.

(34) He, F.; Liang, N. N.; Mu, L.; Pan, Q. H.; Wang, J.; Reeves, M. J.; Duan, C. Q. Molecules. 2012, 17, 1571-1601.

(35) Alcalde-Eon, C.; Escribano-Bailon, M. T.; Santos-Buelga, C.; Rivas-Gonzalo, J. C. Anal. Chim. Acta 2006, 563, 238-254.

(36) Pati, S.; Losito, I.; Gambacorta, G.; La Notte, E.; Palmisano, F.; Zambonin, P. G. J. Mass Spectrom. 2006, 41, 861-871.

(37) Jackson, R. S. In Wine Science: Principles and Applications; Elsevier-Academic Press: Oxford, UK, 2008.

(38) Nave, F.; Teixeira, N.; Mateus, N.; de Freitas, V. Food Chem. 2010, 121, 1129-1138.

(39) Salas, E.; Fulcrand, H.; Meudec, E. J. Agric. Food Chem. 2003, 51, 7951-7961.

(40) Salas, E.; Atanasova, V.; Poncet-Legrand, C.; Meudec, E.; Mazauric, J. P.; Cheynier, V. Anal. Chim. Acta. 2004, 513, 325-332. 
(41) He, F.; Liang, Pan, Q. H.; Ying, S.; Duan, C. Q. Molecules. 2008, 13, 3007-3032.

(42) Sanchez-Ilarduya, M. B.; Sanchez-Fernandez, C.; Viloria-Bernal, M.; Lopez-

Marquez, D. M.; Berrueta, L. A.; Gallo, B.; Vicente, F. Aust. J. Grape Wine Res. 2012, 18, 203-214.

(43) Sanchez-Ilarduya, M. B.; Sanchez-Fernandez, C.; Garmon-Lobato, S.; Abad-Garcia, B.; Berrueta, L. A.; Gallo, B.; Vicente, F. Talanta. 2014, 121, 81-88.

(44) Francia-Aricha, E. M.; Guarra, M. T.; Rivas-Gonzalo, J. C.; Santos-Buelga, C. J. Agric. Food Chem. 1997, 45, 2262.

(45) Timberlake, C. F.; Bridle, P. Am. J. Enol. Vitic. 1976, 27, 97-105.

(46) Marques, A.; Duenas, M.; Serratosa, M. P.; Merida, J. J. Chem. 2012, 2013, 1-7.

(47) Vivar-Quintana, A. M.; Santos-Buelga, C.; Francia-Aricha, E.; Rivas-Gonzalo, J. C. Food Sci. Technol. Int. 1999, 5, 347-352.

(48) Mateus, N.; Silva, A. M. S.; Santos-Buelga, S.; Rivas-Gonzalo, J. C. J. Agric. Food Chem. 2002, 50, 2110-2116.

(49) Asenstorfer, R. E.; Hayasaka, Y.; Jones, G. P. J. Agric. Food Chem. 2001, 49, $5957-$ 5963.

(50) Monagas, M.; Nunez, N.; Bartolome, B.; Gomez-Cordoves, C. Am. J. Enol. Vitic 2003, 54, 163-169.

(51) Cruz, L.; Teixeira, N.; Silva, A. M.; Mateus, N.; Borges, J.; De Freitas, V. J. Agric. Food Chem. 2008, 56, 10980-10987.

(52) von Baer, D.; Rentzsch, M.; Hitschfeld, M. A.; Mardones, C.; Vergara, C.;

Winterhalter, P. Anal. Chim. Acta. 2008, 621, 52-56.

(53) de Freitas, V.; Mateus, N. Anal Bioanal Chem. 2011, 401, 1463-1473.

(54) Romero, C.; Bakker, J. J. Agric. Food Chem. 1999, 47, 3130-3139.

(55) Schwarz, M.; Jerz, G.; Winterhalter, P. Vitis 2003, 42, 105-106.

(56) He, J.; Oliveira, J.; Silva, A. M.; Mateus, N.; De Freitas, V. J. Agric. Food Chem. 2010, 58, 8814-8819. 


\section{SUPPORTING INFORMATION FOR THE MANUSCRIPT:}

\section{Comprehensive two-dimensional HILIC $\times$ RP-LC-UV-MS analysis of anthocyanins and derived pigments in red wine}

Chandré M. Willemse', Maria A. Stander ${ }^{2,3}$, Jochen Vestner ${ }^{4}$, Andreas G.J. Tredoux ${ }^{1}$, André de Villiers ${ }^{1 *}$

${ }^{1}$ Stellenbosch University, Department of Chemistry and Polymer Science, Private Bag X1, Matieland 7602, South Africa

${ }^{2}$ Stellenbosch University, Central Analytical Facility, Private Bag X1, Matieland 7602, South Africa

${ }^{3}$ Stellenbosch University, Department of Biochemistry, Private Bag X1, Matieland 7602, South Africa

${ }^{4}$ Center of Analytical Chemistry and Microbiology, Department of Microbiology and Biochemistry, Hochshule Geisenheim, Germany 
(c)

$\mathrm{R}_{3}, \mathrm{R}_{4}=\mathrm{H}$ or

$\mathrm{R}_{5}=\mathrm{R}_{3} \quad$ or

$\mathrm{R}_{6}=\mathrm{R}_{3}$ or $\mathrm{H}$

acetyl

or<smiles>CC(=O)/C=C/c1ccc(O)c(O)c1</smiles>

or<smiles>CC(=O)C=Cc1ccc(O)cc1</smiles>
$p$-coumaroyl<smiles></smiles>

Vitisin A derivatives
Dp: $\mathrm{R}_{1}=\mathrm{OH}, \mathrm{R}_{2}=\mathrm{OH}, \mathrm{R}_{3}=\mathrm{R}_{4}=\mathrm{H}$

Cy: $\quad \mathrm{R}_{1}=\mathrm{OH}, \mathrm{R}_{2}=\mathrm{R}_{3}=\mathrm{R}_{4}=\mathrm{H}$

Pel: $\mathrm{R}_{1}=\mathrm{R}_{2}=\mathrm{R}_{3}=\mathrm{R}_{4}=\mathrm{H}$

Pt: $\quad \mathrm{R}_{1}=\mathrm{OCH}_{3}, \mathrm{R}_{2}=\mathrm{OH}, \mathrm{R}_{3}=\mathrm{R}_{4}=\mathrm{H}$

Pe: $\mathrm{R}_{1}=\mathrm{OCH}_{3}, \mathrm{R}_{2}=\mathrm{R}_{3}=\mathrm{R}_{3}=\mathrm{H}$

Mv: $\mathrm{R}_{1}=\mathrm{OCH}_{3}, \mathrm{R}_{2}=\mathrm{OCH}_{3}, \mathrm{R}_{3}=\mathrm{R}_{4}=\mathrm{H}$<smiles>[R6]c1cc(-c2c(O)[o+]c3cc(O)cc4c3c2C=CO4)cc(Br)c1O</smiles>

Vitisin B derivatives

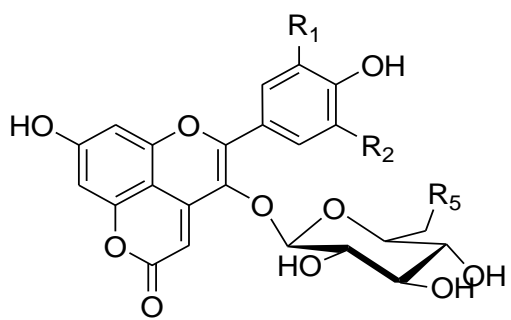

Anthocyanin oxovitisin derivatives

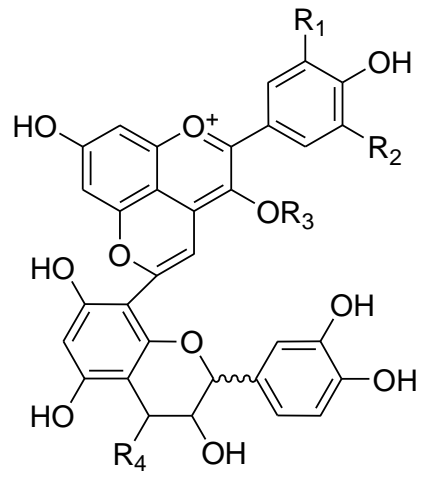

Anthocyanin vinylflavanol condensation products<smiles></smiles>

ANC-vinylphenol

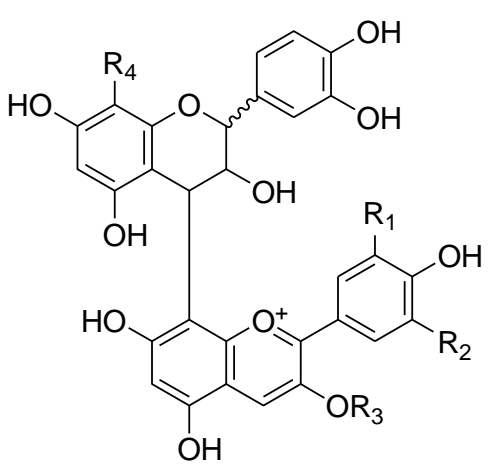

Direct anthocyanin tannin adducts (F-A)<smiles></smiles>

ANC-vinylguaiacol<smiles></smiles>

Direct anthocyanin tannin adducts (A-F)<smiles></smiles>

ANC-vinylcatechol<smiles>[R5]c1cc2c(cc1O)C(O)=CC(OC)=C1OC(c3cc([R6])c(O)c([R5])c3)=C1O2</smiles>

Acetaldehyde mediated tannin adducts<smiles>[R6]c1cc(-c2oc3cc(O)cc4c3c2C=C(c2cc(OC)c(O)c(OC)c2)O4)cc(Br)c1O</smiles>

ANC-vinylsyringol 


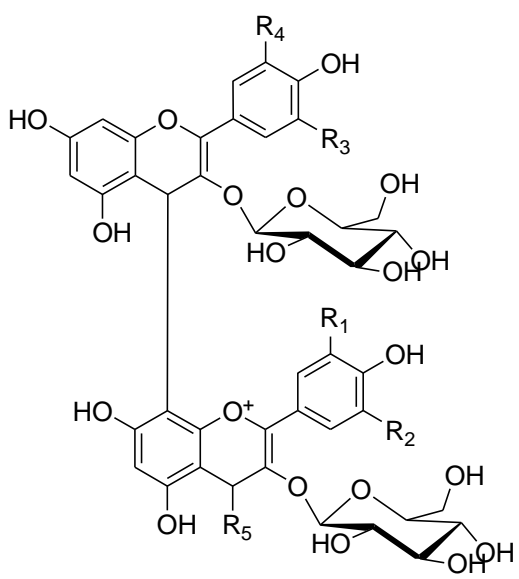

Oligomeric Anthocyanins

Figure S1. Structures of anthocyanins and their derived pigments detected in wine. Abbreviations: $\mathrm{Dp}=$ delphinidin $; \mathrm{Cy}=$ cyanidin $; \mathrm{Pt}=$ Petunidin $; \mathrm{Pe}=$ Peonidin $; \mathrm{Mv}=$ malvidin; $\mathrm{ANC}=$ anthocyanidin . 


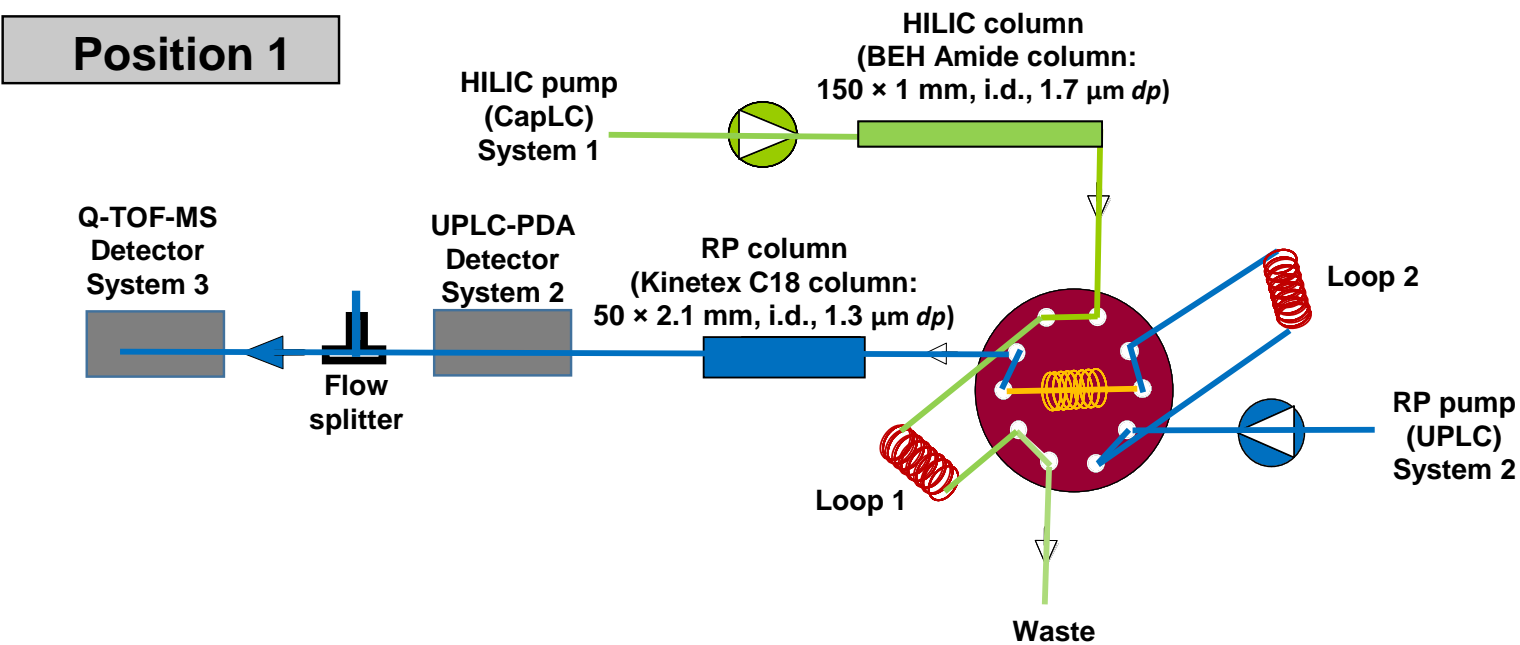

\section{Position 2}

HILIC column

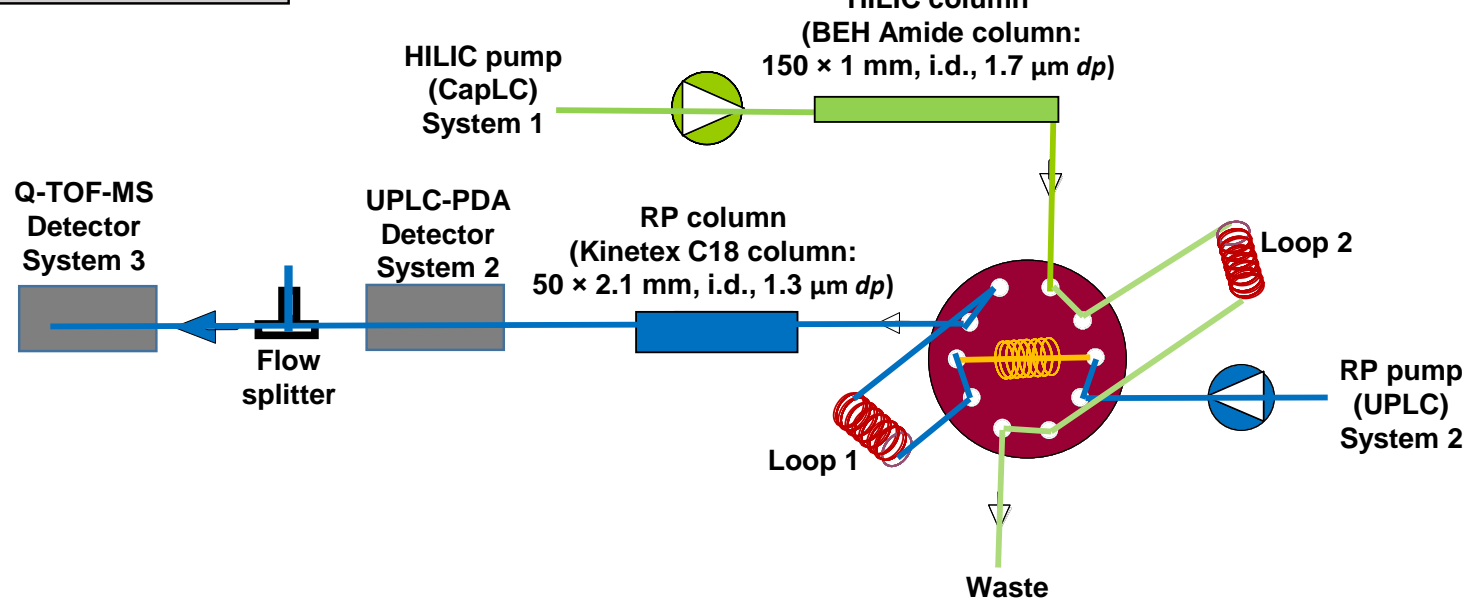

Figure S2: Schematic representation of the instrumental configuration used for on-line HILIC $\times$ RP-LC-UV-MS analyses. 

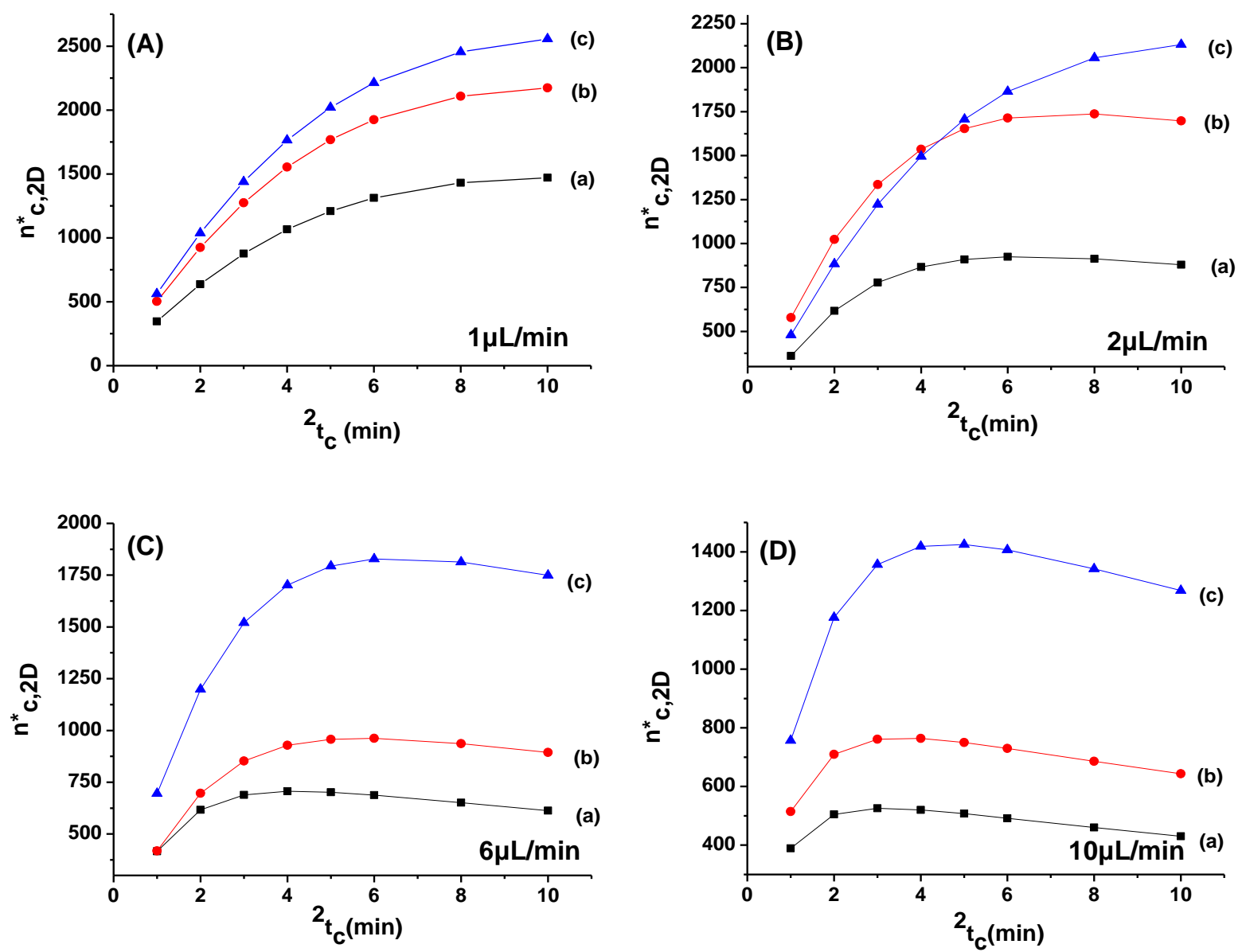

Figure S3: Plots of corrected practical 2-D peak capacity $\left(n^{*}, 2 D\right)$ as a function of second dimension cycle time $\left({ }^{2} t_{c}\right)$ for various first dimension flow rates and gradient times: (A) flow rate $1 \mu \mathrm{L} / \mathrm{min}$, gradient times (a) $230 \mathrm{~min}$, (b) $295 \mathrm{~min}$ and (c) $406 \mathrm{~min}$; (B) flow rate $2 \mu \mathrm{L} / \mathrm{min}$, gradient times (a) $156 \mathrm{~min}$, (b) $217 \mathrm{~min}$ and (c) $376 \mathrm{~min}$; (C) flow rate $6 \mu \mathrm{L} / \mathrm{min}$, gradient times (a) $72 \mathrm{~min}$, (b) $126 \mathrm{~min}$ and (c) $217 \mathrm{~min}$; (D) flow rate $10 \mu \mathrm{L} / \mathrm{min}$, gradient times (a) $50 \mathrm{~min}$, (b) 75 min and (c) 150 min. 


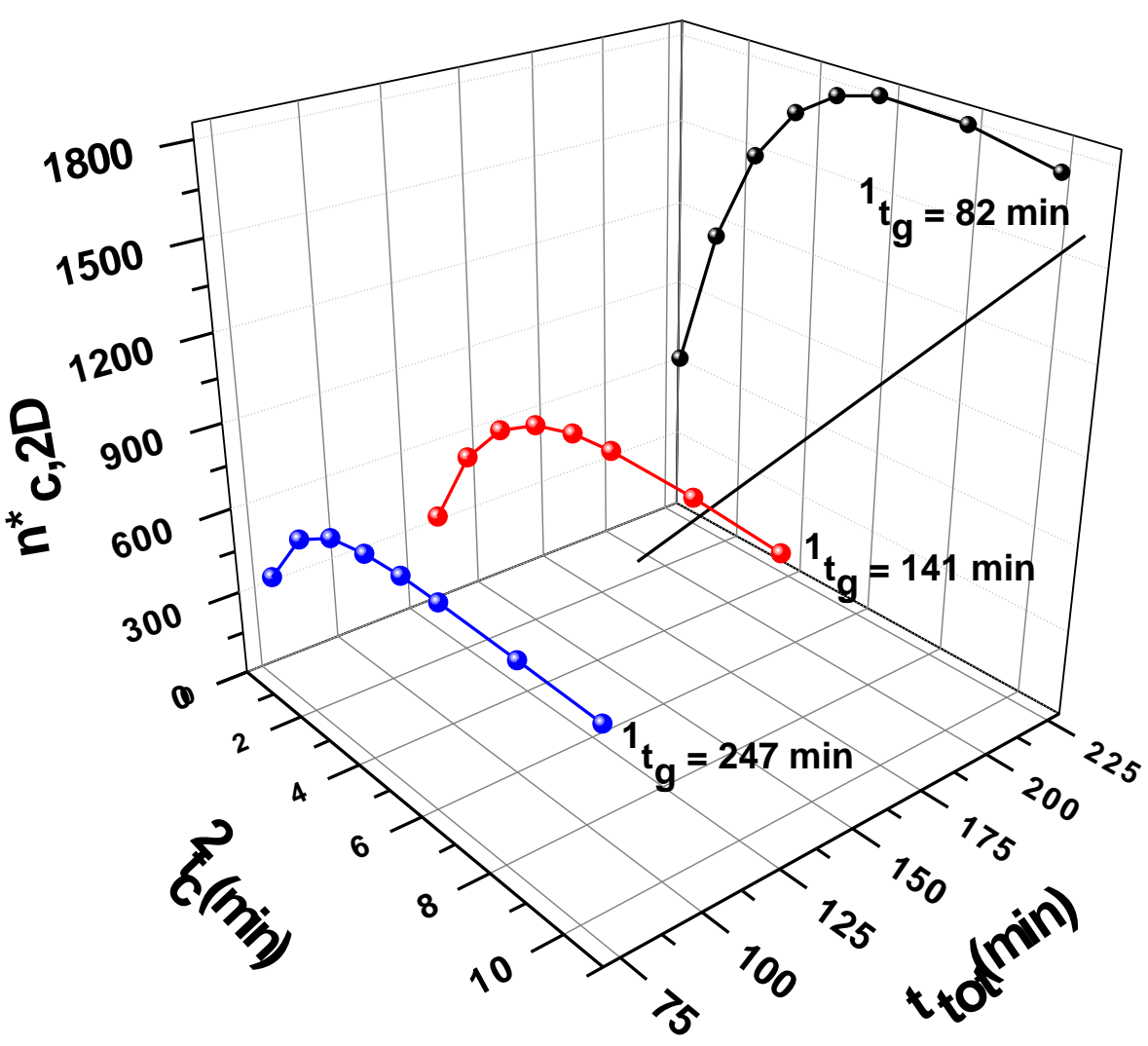

Figure S4: Plot of corrected practical 2-D peak capacity as a function of total analysis time and second dimension cycle time at a first dimension flow rate of $6 \mu \mathrm{L} / \mathrm{min}$. Values are plotted for first dimension gradient times of: $82 \mathrm{~min}$ (blue), $141 \mathrm{~min}$ (red) and $247 \mathrm{~min}$ (black). 

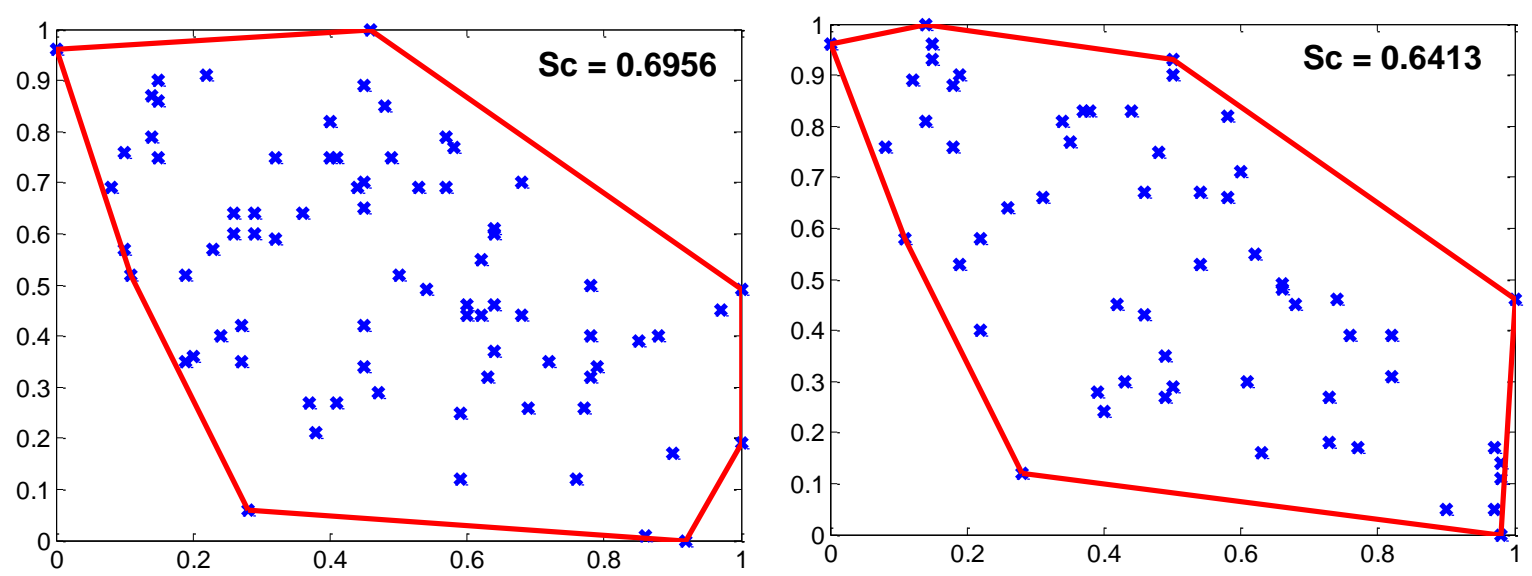

Figure S5: Determination of the surface coverage of the two-dimensional separation space using the convex hull method for anthocyanins and derived pigments identified in (a) 2013 and (b) 2008 Pinotage wines by HILIC $\times$ RP-LC-MS analysis. 
Table S1: Summary of parameters used to calculate the practical peak capacity of on-line HILIC $\times$ RP-LC analysis of red wine pigments.

\begin{tabular}{lc}
\hline Parameters & Value \\
\hline${ }^{\text {a}}$ HILIC peak capacity, ${ }^{1} n_{\mathrm{c}}$ & 22 \\
${ }^{\text {a}} \mathrm{RP}$ peak capacity (average), ${ }^{2} n_{\mathrm{c}}$ & 63 \\
${ }^{\mathrm{b}}$ Surface coverage, $\left(\mathrm{SC}_{\mathrm{CH}}, f_{\mathrm{c}}\right)$ & $0.6821^{\mathrm{c}}$ \\
${ }^{\mathrm{d}}$ Theoretical peak capacity, $n_{\mathrm{c}, 2 \mathrm{D}}$ & 1386 \\
${ }^{\mathrm{e}}$ Under-sampling correction factor, $\beta$ & 1.02 \\
fPractical peak capacity, $n^{*}{ }_{\mathrm{c}, 2 \mathrm{D}}$ & $889^{\mathrm{c}}$ \\
Total analysis time $(\mathrm{min})$ & 408 \\
\hline
\end{tabular}

${ }^{\mathrm{a} C a l c u l a t e d}$ according to ${ }^{27}$, equation 3 .

${ }^{\mathrm{b} C a l c u l a t e d}$ according to the convex hull method $^{31}$.

${ }^{c}$ Values are the average for the 2013 and 2008 wines.

${ }^{\mathrm{d}} n_{\mathrm{c}, 2 \mathrm{D}}={ }^{1} n_{\mathrm{c}} \times{ }^{2} n_{\mathrm{c}}$

${ }^{\mathrm{e}} \beta=$ calculated according to $\mathrm{Li}$ et al. ${ }^{29}$.

${ }^{\mathrm{f}}$ Calculated according to equation 4 , corrected for lack of orthogonality by using $\mathrm{SC}_{\mathrm{CH}}{ }^{31}$ and under-sampling according to ${ }^{29}$. 